\begin{tabular}{|c|c|c|c|c|c|c|}
\hline 0 & $n$ & & & $\mathrm{~S}$ & $l$ & 0 \\
\hline$n$ & e & $S$ & $S$ & & a & $n$ \\
\hline$i$ & $n$ & $h$ & a & b & i & $t$ \\
\hline$n$ & 9 & & & $t$ & i & $\mathrm{m}$ \\
\hline
\end{tabular}





\title{
ON SLOWNESS AND INHABITING TIME
}

\author{
by Sahel Tahvildari \\ Bachelor of Architectural Science, Ryerson \\ University, 2014 \\ A thesis \\ presented to Ryerson University \\ in partial fulfilment of the \\ requirements for the degree of \\ Master of Architecture \\ in the program of \\ Architecture \\ Toronto, Ontario, Canada, 2017 \\ (C) Sahel Tahvildari, 2017
}




\section{AUTHOR'S DECLARATION FOR ELECTRONIC SUBMISSION OF A THESIS}

I hereby declare that I am the sole author of this thesis. This is a true copy of the thesis, including any required final revisions, as accepted by my examiners.

I authorize Ryerson University to lend this thesis to other institutions or individuals for the purpose of scholarly research.

I further authorize Ryerson University to reproduce this thesis by photocopying or by other means, in total or in part, at the request of other institutions or individuals for the purpose of scholarly research.

I understand that my thesis may be made electronically available to the public. 
On Slowness and Inhabiting Time

Sahel Tahvildari

Master of Architecture, 2017

Architecture Program, Ryerson University

Abstract

As a result of today's accelerated culture, the omnipresence of speed has taken over everyday life. The influence of flexibility and efficiency within contemporary architecture parallels the need for speed. The primacy of speed has hindered the temporal and cognitive presence in everyday life; shifting the human experience of the world from lived to measured. Through the reconsideration of slowness as an embodied experience, the rhythmic passage of the body through space establishes a mindful physical presence as opposed to modern distraction. In architecture, spatial and perceptual unfolding intertwines perceiver and perceived to sequentially reveal the layers of experience through passage. Within slowness, visual fluidity creates a viscous and emotive relationship between mind, body, form, and movement. The relationship between speed, time, and presence, guides the mindful body through a layered procession which unfolds in the creation of an architecture of slowness. 
Acknowledgments

John Cirka

Vincent Hui

Colin Ripley

My Family

Matthew

Jessica, Krystyna, Giovanna, Demitri, Mike 
table of contents

Author's Declaration

Abstract

Acknowledgments

Dedication

List of Figures

Introduction

01

Speed Theory

04

1.1 The Acceleration of Everything

05

1.2 Contemporary Speed

08

1.3 The Speed of Reception

10

1.3.1 Montage as Stills

\section{$02 \quad$ Frame Speed}

24

2.1 Cinema and Time

2.2 Cinematic Montage

2.2.1 Battleship Potemkin 32

2.2.2 La Jetée 34

2.2.3 Mirror 36

2.2.4 Taste of Cherry 38

2.3 Lived Frame 40

2.3.1 Lived Montage $\quad 46$

$03 \quad$ Slow Movement 56

3.1 Time Poverty 57

3.2 Slow Culture 60

\section{$04 \quad$ Slow Medium}

4.1 Duration

4.2 Presence 68

4.3 Place 
5.1 Architecture as Vehicle for Inhabiting Time

5.2 Generators of Slowness 82

5.3 House on the Coast 86

\subsubsection{Physis \\ 5.3.2 Sequence 01 \\ 5.3.3 Sequence 02} 86 91 105

5.4 Conclusion

Appendix A

Moments of Slowness in Japan

Appendix B

Sequence 01

Appendix C

Sequence 02

Appendix D

Temporal Representation

Bibliography 
Figure 1a The Passage of Time, 2016

Source Sahel Tahvildari

Figure 1b Endless House, 1947 - 1960, Friedrick Kiesler

Source "Endless House/Friedrick Kiesler." April 11, 2011. Accessed December 7, 2016.

http://www.archdaily.com/126651/ad-classics-endlesshouse-friedrick-kiesler

Figure 2 The Dark Side of The City, 2015, André.G

Source "The Dark Side of The City." April 18, 2015. Accessed December 7, 2016.

https://www.flickr.com/photos/125114487a

N06/16875658853

Figure 3 Futurist Portrait of Marinetti, 1930, Luxardo Giorgio

Source "Marinetti." Accessed April 13, 2017.

www.architetturafuturista.it/marinett.htm

Figure 4 Painted Speed

a Nude Descending a Staircase, 1912, Marcel Duchamp

Source "Nude Descending a Staircase (No. 2)." Accessed September 26, 2016.

http://www.philamuseum.org/collections/ permanent/51449.html

b Dynamism of a Dog on a Leash, 1912, Giacomo Balla

Source "Dynamism of a Dog on a Leash." Accessed September 26, 2016.

http://www.independent.co.uk/arts-entertainment/art/ great-works/great-works-dynamism-of-a-dog-on-a-leash1912-giacomo-balla-1781174.html

Figure 5 Photographed Speed

a Walking, Under Various Conditions, 1881, Eadweard Muybridge 
Source "A Body in Motion." July 6, 2012. Accessed on November 22 , 2016.

https://raunerlibrary.blogspot.ca/2012/07/body-in-motion. html?m=1

Edited by Sahel Tahvildari

b Chronophotograph of Walking Woman, 1893, Étienne-Jules Marey

Source "Etienne Jules Marey." Accessed on September 29, 2016. http://cinematics-jean.blogspot.ca/2011/03/etienne-julesmarey.html

Figure 6 The Bride Stripped Bare by Her Bachelors, Even or Delay in Glass, 1915-1923, Marcel Duchamp

Source "The Bride Stripped Bare by Her Bachelors, Even (The Large Glass)." Accessed April 14, 2017.

http://www.philamuseum.org/collections/ permanent/54149.html

Figure 7 Stills: Bullet Train in Japan, 2016

Source Sahel Tahvildari

Figure 8 Speed Perception, 2017

Source Sahel Tahvildari

Figure 9 Montage as Stills; Accelerated, 2016

Source Sahel Tahvildari

Figure 10 Montage as Stills; Slowed, 2016

Source Sahel Tahvildari

Figure 11 Montage as Stills; Paused, 2016

Source Sahel Tahvildari

Figure 12 Stills: La Jetée, 1962, Chris Marker

Source Sahel Tahvildari

Figure 13 Imaginary Prisons, 1925, Giovanni Battista Piranesi

Source "Giovanni Battista Piranesi." Accessed July 31, 2017.

https://en.wikipedia.org/wiki/Giovanni_Battista_Piranesi

Figure 14 The Kuleshov Effect, 1918, Lev Kuleshov

Source "Continually Confusing - Part Three." November 24, 2015.

Accessed April 14, 2017.

http://garethlewis3dca.blogspot.ca/2015/11/continuallyconfusing-part-three.html

Figure 15 Battleship Potemkin, 1925, Sergei Eisenstein Source "Battleship Potemkin (1925)." January 30, 2013. Accessed on July 31, 2017.

https://unaffiliatedcritic.com/2013/01/battleship-potemkin1925-independent-study-in-world-cinema/ 
Figure 16

Source

Figure 17
Source

Figure 18

Source

ii

Source

ii

Source

iv

Source

$v$

Source

vi

Source

Mother Carrying Dead Boy

"Battleship Potemkin." December 14, 2014. Accessed July 31, 2017.

https://film-grab.com/2014/12/17/battleship-potemkin/

Figure 19 Stills: La Jetée

i Man in Agony 1

Source "La Jetée." June 5, 2015. Accessed July 31, 2017. https://film-grab.com/2015/06/05/la-jetee/

ii Man in Agony 2

Source "La Jetée." June 5, 2015. Accessed July 31, 2017. https://film-grab.com/2015/06/05/la-jetee/

iii Field

Source “La Jetée." June 5, 2015. Accessed July 31, 2017. https://film-grab.com/2015/06/05/la-jetee/ 


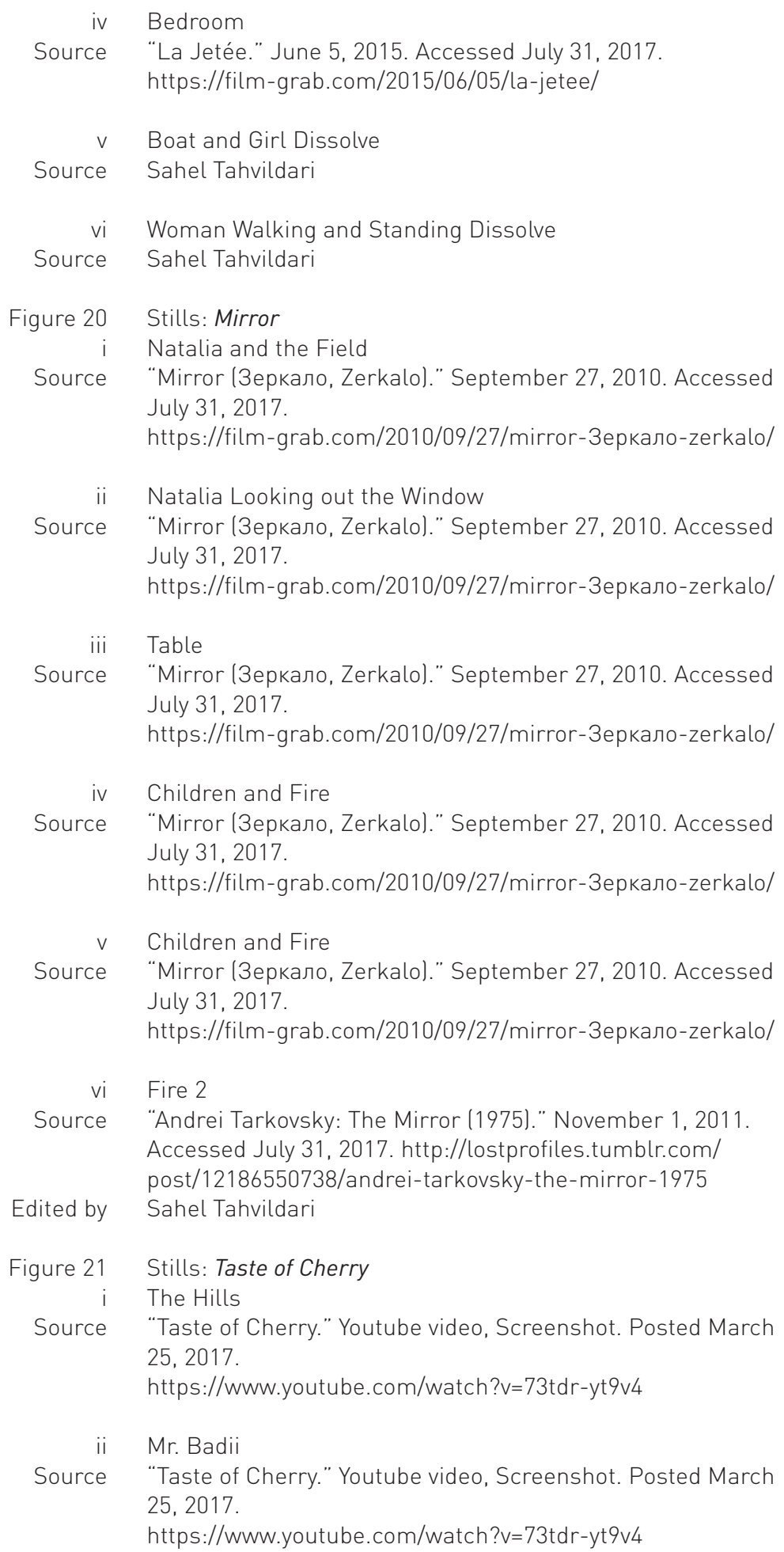




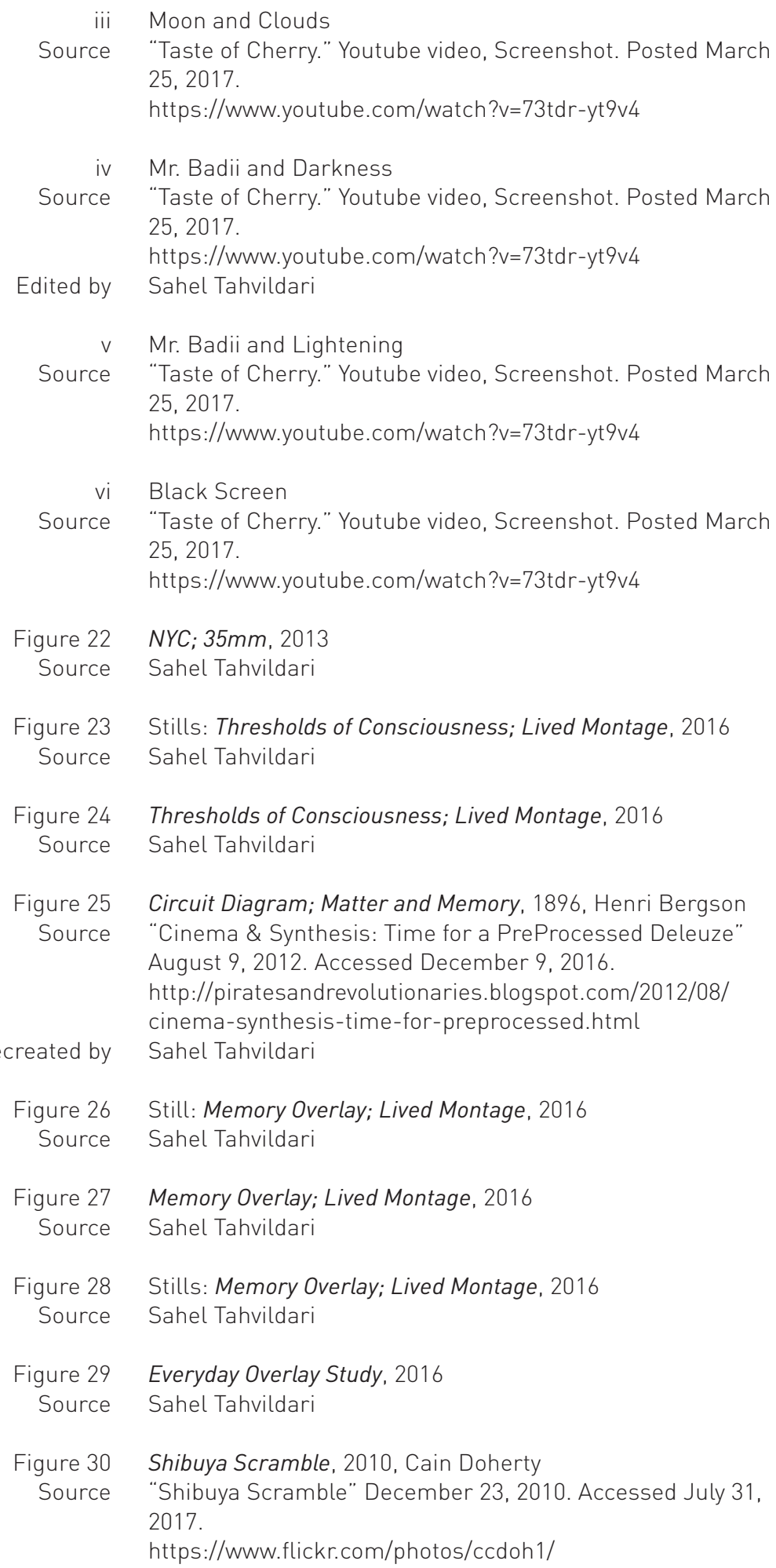

Figure $22 \quad$ NYC; 35mm, 2013

Source Sahel Tahvildari

Figure 23 Stills: Thresholds of Consciousness; Lived Montage, 2016 Source Sahel Tahvildari

Figure 24 Thresholds of Consciousness; Lived Montage, 2016

Source Sahel Tahvildari

Figure 25 Circuit Diagram; Matter and Memory, 1896, Henri Bergson

Source "Cinema \& Synthesis: Time for a PreProcessed Deleuze" August 9, 2012. Accessed December 9, 2016. http://piratesandrevolutionaries.blogspot.com/2012/08/ cinema-synthesis-time-for-preprocessed.html Sahel Tahvildari

Figure 26 Still: Memory Overlay; Lived Montage, 2016 Source Sahel Tahvildari

Figure 27 Memory Overlay; Lived Montage, 2016

Source Sahel Tahvildari

Figure 28 Stills: Memory Overlay; Lived Montage, 2016

Source Sahel Tahvildari

Figure 29 Everyday Overlay Study, 2016

Source Sahel Tahvildari

Figure 30 Shibuya Scramble, 2010, Cain Doherty Source "Shibuya Scramble" December 23, 2010. Accessed July 31, 2017.

https://www.flickr.com/photos/ccdoh1/

Recreated by 
Figure 31 Pattinatori; Skaters, 1953, Mario de Biasi

Source "Pattinatori; Skaters." Accessed July 31, 2017.

http://www.artnet.com/artists/mario-de-biasi/pattinatoriskaters-TwdiW4fJwaZYeq-nNIOwCg2

Figure 32 Roman Holiday, 1953, William Wyler

Source "Roman Holiday." March 1, 2017. Accessed July 31, 2017.

https://film-grab.com/2017/03/01/roman-holiday/

Figure 33 Christmas Lunch, 1950, Unknown

Source "Childhood Memories of Christmas Lunch." December 24, 2014. Accessed July 31, 2017.

http://www.adelaiderememberwhen.com.au/childhood-

memories-of-christmas-lunch/

Figure 34 Mother and Son, 1997, Aleksandr Sokurov

Source "Mother and Son (1997)." August 4, 2013. Accessed July 31, 2017.

http://www.boloji.com/index.cfm?md=Content\&sd=Articles \&ArticlelD=14755

Figure 35

Source

Walkers Emerge From The Fog, 2014, Paudle Fearon

"Walkers Emerge From The Fog." December 22, 2014.

Accessed July 31, 2017.

https://www.flickr.com/photos/paudiefearon/

Figure 36 Slow House, 1990, Diller and Scofidio

Source "Slow House Diller Scofidio Plans." Accessed July 30, 2017.

http://design-net.biz/houseplans/slow_house_diller_

scofidio_plans.html

Figure 37 Sequence, 2006, Richard Serra

Source "Sequence." Accessed August 1, 2017.

https://www.moma.org/interactives/exhibitions/2007/serra/

flash.html

Edited by Sahel Tahvildari

Figure 38 Saint Benedict's Chapel, 1988, Peter Zumthor

Source Sahel Tahvildari

Figure 39 Blons Community Centre, 2005, Bruno Klomfar

Source Sahel Tahvildari

Figure 40 Jewish Museum Berlin, 1999, Peter Zumthor

a Light

Source "Jewish Museum Berlin." December 15, 2008. Accessed

August 1, 2017.

https://www.flickr.com/photos/copelaes/3152561112/

Edited by Sahel Tahvildari 
b Darkness

Source Arends, Jasper. “Jewish Museum Berlin.” November 13, 2011.Accessed August 1, 2017.

https://www.flickr.com/photos/jazzplanet/6391162985/in/

photostream/

Edited by Sahel Tahvildari

Figure 41 Volksschule Doren; Doren Primary School, 2003, Curkowicz Nachbaur

Source Sahel Tahvildari

Figure 42 The Glasgow School of Arts, 1999, Steven Holl

Source "Steven Holl completes extension to Mackintosh's Glasgow School of Art." March 6 2014. Accessed on August 2, 2017. https://www.dezeen.com/2014/03/06/glasgow-school-ofart-reid-building-steven-holl/

Edited by Sahel Tahvildari

Figure 43 Temppeliaukion Kirkko; Rock Church, 1969, Timo and Tuomo Suomalainen

Source Sahel Tahvildari

Figure 44 Montage Study, 2016

a Wire Model

Source Sahel Tahvildari

b Experience of the Wire Model

Source Sahel Tahvildari

Figure 45 Japan, 2016

a Shiba Ryōtarō Memorial Museum, 2000 - 2001, Tadao Ando

Source Sahel Tahvildari

b Nanzen-ji Temple

Source Sahel Tahvildari

c Church of Light, 1989, Tadao Ando

Source Sahel Tahvildari

d Water Temple, 1991, Tadao Ando

Source Sahel Tahvildari

e Ryōan-Ji Temple and Rock Garden

Source Sahel Tahvildari

Figure 46 San Francisco; 35mm, 2017

a of Smell

Source Matthew Gelowitz

b of Sound

Source Matthew Gelowitz 


\begin{tabular}{|c|c|}
\hline c & of Light and Shadow \\
\hline Source & Matthew Gelowitz \\
\hline$d$ & of Sight \\
\hline Source & Matthew Gelowitz \\
\hline Figure 47 & Sequence 01, 2017 \\
\hline Source & Sahel Tahvildari \\
\hline Figure 48 & Theory to Design; Sequence 01, 2017 \\
\hline Source & Sahel Tahvildari \\
\hline Figure 49 & Generators of Slowness; Sequence 01, 2017 \\
\hline Source & Sahel Tahvildari \\
\hline Figure 50 & Passage; Sequence 01, 2017 \\
\hline Source & Sahel Tahvildari \\
\hline Figure 51 & Orthographic Drawings, 2017 \\
\hline a & Roof, Sequence 01 \\
\hline Source & Sahel Tahvildari \\
\hline$b$ & Level 01, Sequence 01 \\
\hline Source & Sahel Tahvildari \\
\hline c & Level 02, Sequence 01 \\
\hline Source & Sahel Tahvildari \\
\hline$d$ & Level 03, Sequence 01 \\
\hline Source & Sahel Tahvildari \\
\hline e & Level 04, Sequence 01 \\
\hline Source & Sahel Tahvildari \\
\hline f & Level 05, Sequence 01 \\
\hline Source & Sahel Tahvildari \\
\hline$g$ & Cross Section Study, Section aa and Section bb, Sequence 01 \\
\hline Source & Sahel Tahvildari \\
\hline $\mathrm{h}$ & Cross Section Study, Section cc and Section dd, Sequence 01 \\
\hline Source & Sahel Tahvildari \\
\hline i & Cross Section Study, Section ee and Section ff, Sequence 01 \\
\hline Source & Sahel Tahvildari \\
\hline j & Cross Section Study, Section gg and Section hh, Sequence 01 \\
\hline Source & Sahel Tahvildari \\
\hline k & Cross Section Study, Section ii and Section jj, Sequence 01 \\
\hline Source & Sahel Tahvildari \\
\hline
\end{tabular}


l Cross Section Study, Section kk and Section II, Sequence 01

Source Sahel Tahvildari

m Section $\mathrm{mm}$, Sequence 01

Source Sahel Tahvildari

Figure 52 Sequence 02, 2017

Source Sahel Tahvildari

Figure 53 Spatial and Perceptual Collision, 2017

a Level 01 - Level 02, Sequence 02

Source Sahel Tahvildari

b Level 03 - Level 04, Sequence 02

Source Sahel Tahvildari

Figure 54 Passage; Sequence 02, 2017

Source Sahel Tahvildari

Figure 55 Everyday Filmic Passage; Sequence 02, 2017

Source Sahel Tahvildari

Figure 56 Spatial Overlap and Axis Study, 2017

Source Sahel Tahvildari

Figure 57 Orthographic Drawings, 2017

a Roof, Sequence 02

Source Sahel Tahvildari

b Level 01, Sequence 02

Source Sahel Tahvildari

c Level 02, Sequence 02

Source Sahel Tahvildari

d Level 03, Sequence 02

Source Sahel Tahvildari

e Level 04, Sequence 02

Source Sahel Tahvildari

f Section aa, Sequence 02

Source Sahel Tahvildari

g Section bb, Sequence 02

Source Sahel Tahvildari

h Section cc, Sequence 02

Source Sahel Tahvildari

i Section dd, Sequence 02

Source Sahel Tahvildari 


\begin{tabular}{|c|c|}
\hline j & Section ee, Sequence 02 \\
\hline k & Section ff, Sequence 02 \\
\hline Source & Sahel Tahvildari \\
\hline l & Section gg, Sequence 02 \\
\hline Source & Sahel Tahvildari \\
\hline m & East Elevation, Sequence 02 \\
\hline Source & Sahel Tahvildari \\
\hline$n$ & West Elevation, Sequence 02 \\
\hline Source & Sahel Tahvildari \\
\hline 0 & North Elevation, Sequence 02 \\
\hline Source & Sahel Tahvildari \\
\hline Figure 58 & Everyday Inhabitance, 2017 \\
\hline a & Wake Up, Sequence 02 \\
\hline Source & Sahel Tahvildari \\
\hline b & Breakfast, Sequence 02 \\
\hline Source & Sahel Tahvildari \\
\hline c & Work, Sequence 02 \\
\hline Source & Sahel Tahvildari \\
\hline$d$ & Garden, Sequence 02 \\
\hline Source & Sahel Tahvildari \\
\hline e & Dinner, Sequence 02 \\
\hline Source & Sahel Tahvildari \\
\hline$f$ & Lounge, Sequence 02 \\
\hline Source & Sahel Tahvildari \\
\hline g & Sleep, Sequence 02 \\
\hline Source & Sahel Tahvildari \\
\hline Figure 59 & Shiba Ryōtarō Memorial Museum, 2016 \\
\hline a & Trail \\
\hline Source & Sahel Tahvildari \\
\hline b & Promenade \\
\hline Source & Sahel Tahvildari \\
\hline c & Books \\
\hline Source & Sahel Tahvildari \\
\hline
\end{tabular}




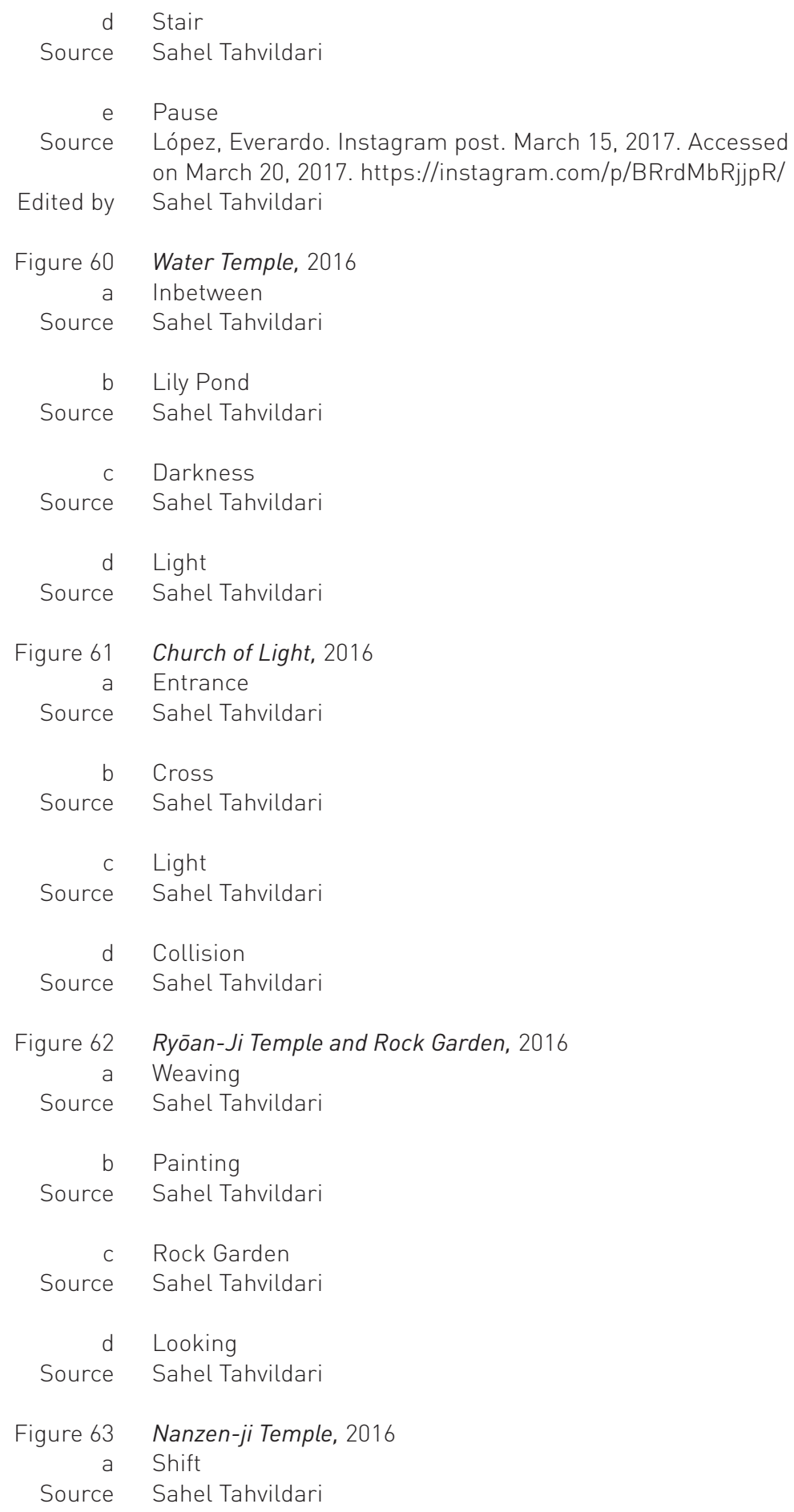

Figure 63 Nanzen-ji Temple, 2016

$\begin{aligned} \text { a } & \text { Shift } \\ \text { Source } & \text { Sahel Tahvildari }\end{aligned}$ 


\begin{tabular}{|c|c|}
\hline b & Pond \\
\hline Source & Sahel Tahvildari \\
\hline c & Bamboo \\
\hline Source & Sahel Tahvildari \\
\hline$d$ & Stepping Stones \\
\hline Source & Sahel Tahvildari \\
\hline Figure 64 & Kennin-Ji Temple, 2016 \\
\hline a & Linear \\
\hline Source & Sahel Tahvildari \\
\hline$b$ & Garden \\
\hline Source & Sahel Tahvildari \\
\hline c & Weave \\
\hline Source & Sahel Tahvildari \\
\hline$d$ & Light \\
\hline Source & Sahel Tahvildari \\
\hline Figure 65 & Stills: Sequence 01, 2017 \\
\hline a & Entrance Steps \\
\hline Source & Sahel Tahvildari \\
\hline b & Through the Doorway \\
\hline Source & Sahel Tahvildari \\
\hline c & Foyer \\
\hline Source & Sahel Tahvildari \\
\hline$d$ & Bench Under the Skylight \\
\hline Source & Sahel Tahvildari \\
\hline e & Kitchen and Dining Room \\
\hline Source & Sahel Tahvildari \\
\hline f & Dining Room Skylight \\
\hline Source & Sahel Tahvildari \\
\hline g & Compressed Stair \\
\hline Source & Sahel Tahvildari \\
\hline h & Expansive View of the Terrain \\
\hline Source & Sahel Tahvildari \\
\hline i & Last Bend \\
\hline Source & Sahel Tahvildari \\
\hline Source & $\begin{array}{l}\text { Living Space Corridor } \\
\text { Sahel Tahvildari }\end{array}$ \\
\hline
\end{tabular}




\begin{tabular}{|c|c|}
\hline k & Living Space View \\
\hline Source & Sahel Tahvildari \\
\hline l & Towards Silence \\
\hline Source & Sahel Tahvildari \\
\hline m & Seat Towards Silence \\
\hline Source & Sahel Tahvildari \\
\hline$n$ & Sit in Silence \\
\hline Source & Sahel Tahvildari \\
\hline 0 & Threshold Between Silence \\
\hline Source & Sahel Tahvildari \\
\hline$p$ & The Moment of Silence \\
\hline Source & Sahel Tahvildari \\
\hline Figure 66 & Stills: Sequence 02, 2017 \\
\hline a & Expansive Site \\
\hline Source & Sahel Tahvildari \\
\hline$b$ & Entrance Steps \\
\hline Source & Sahel Tahvildari \\
\hline c & Rock Garden \\
\hline Source & Sahel Tahvildari \\
\hline$d$ & Foyer \\
\hline Source & Sahel Tahvildari \\
\hline e & Foyer Bench \\
\hline Source & Sahel Tahvildari \\
\hline$f$ & Cut Reveal \\
\hline Source & Sahel Tahvildari \\
\hline$g$ & Moment of Pause; Kitchen and Dining Room \\
\hline Source & Sahel Tahvildari \\
\hline h & Descent to Garden \\
\hline Source & Sahel Tahvildari \\
\hline i & Moment of Pause; Garden \\
\hline Source & Sahel Tahvildari \\
\hline j & Bench \\
\hline Source & Sahel Tahvildari \\
\hline k & Moment of Pause; Bench \\
\hline Source & Sahel Tahvildari \\
\hline
\end{tabular}


I Moment of Pause; Overlapped Garden and Living Space

Source Sahel Tahvildari

m Descent to Living Space

Source Sahel Tahvildari

n Moment of Pause; Living Space

Source Sahel Tahvildari

Figure 67 Thickened Procession, 2016

Source Sahel Tahvildari

Figure 68 Conceptual Models, 2017

a Spatial and Planar Collision

Source Sahel Tahvildari

b Orchestrating Silence

Source Sahel Tahvildari

c Orchestrating Chaotic Silence

Source Sahel Tahvildari

Figure 69 Memory Overlay Wire Model, 2016

Source Sahel Tahvildari

Figure 70 Etched Section Study, 2017

Source Sahel Tahvildari 



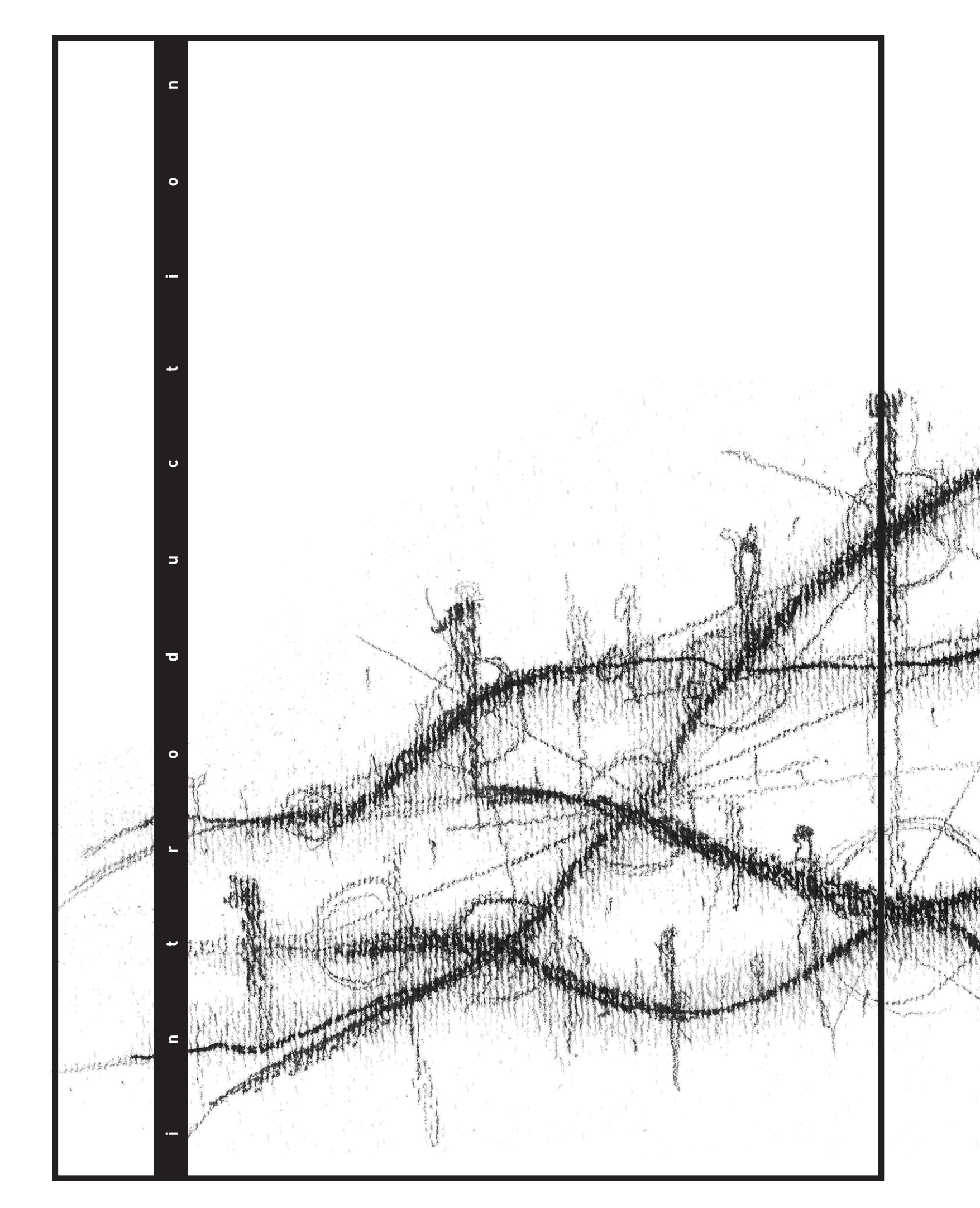


It seems that we have lost our capacity to

dwell in time. Being outside of time is an

aspect of the new homelessness of the

modern man."

f1a

The Passage of Time

2015

Sahel Tahvildari

This thesis explores the

implications and experience of time within architecture

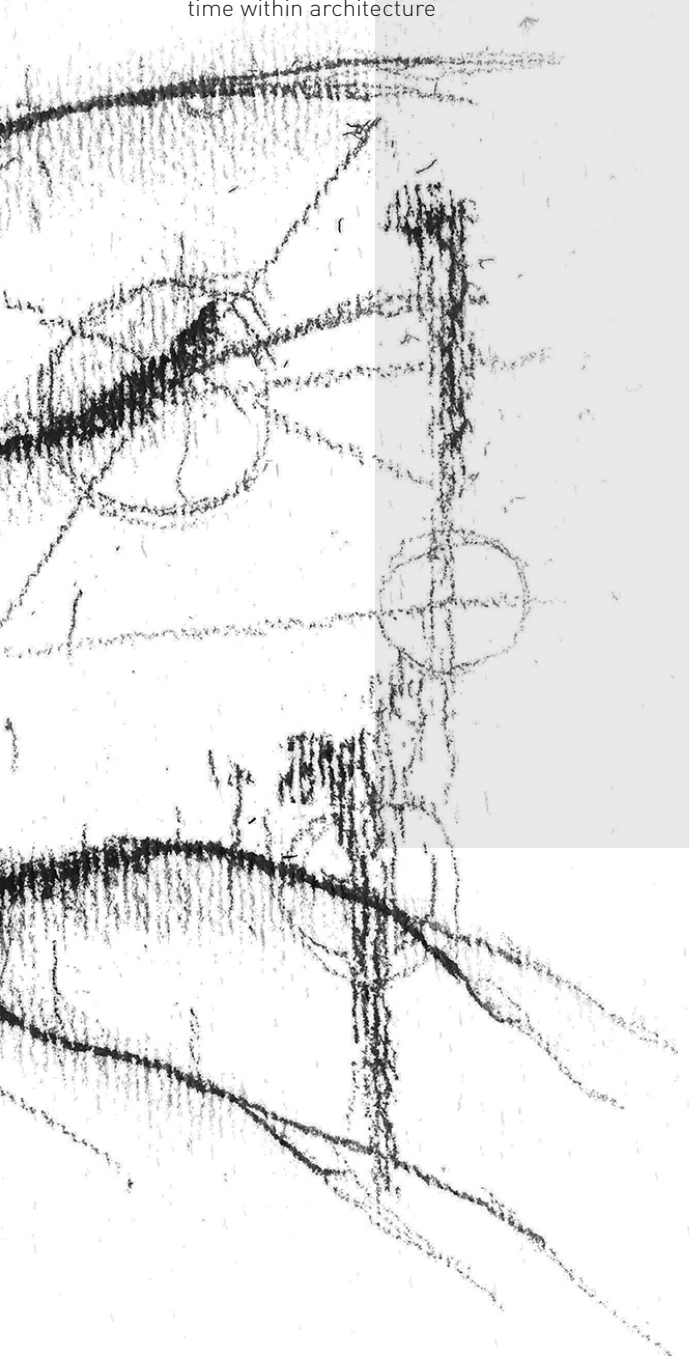

\section{introduction}

Within the contemporary world, the omnipresence of speed characterizes everyday life. Its obsession has led to the absorption of time, whereby the joining of the two deems speed and time as parallel elements which discount experience for measure. The current fixation with speed stems from a sense of urgency through which pace is prioritized. Its drastic effects on the modern world can be seen through the distortion of everyday life where spatial and temporal moments are experienced in a constant state of distraction, one which is physical, cognitive, or both.

The primacy of speed, within the last two centuries has shifted the focus of architecture from experience to pace; this adoption of freneticism within society poses a challenge for architecture as it begins to disassociate perceiver from perceived, or subject from object. This research examines and tests the implications of speed on an individual's experiences of both social and atmospheric space. The findings demonstrate a fragmented association between acceleration and presence. Moreover, the absentminded world has intertwined with contemporary design to support momentary experiences of architectural spectacles, which highlight disjointed memories, over viscous experiences, which expanded space and time to impact the mind and body.

The culture of speed has resulted in a blurring whereby everyday acts are experienced unconsciously. This increase of absentmindedness has built a layer of distraction between experience, and the mind and body. The contemporary lived frame is defined by cuts or 


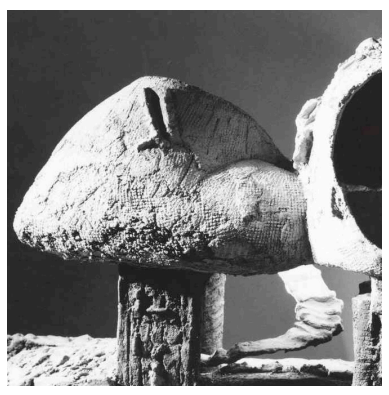

moments rather than a continuous frame sequence. Within film, the varying density of and within frames creates a quickening and slowing of time. This variance is the result of a specific filmic assemblage whereby editing techniques are used to both compress and expand time. Like cinema, architecture is experienced as the summation of and within its parts; its sequential nature establishes a layered sequence that allows for various speeds of reception and interaction within an experience. Through a 'montagist' design process, filmic editing techniques foster an investigation into the perception and reception of architectural frames. Moments of spatial and perceptual collision tumble through space and time in order to traverse the mind and body between varying levels of stimuli. This traversal allows for a contrasting experience of both frenetic and slow speeds; by doing so, the juxtaposition both creates and heightens presence within space.

Presence, is the physical or cognitive state of being present in a specific place or moment. With inhabiting time, the intent is to create architecture that harnesses

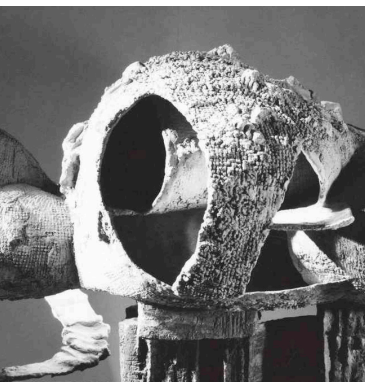
spatial and perceptual collision, whereby, frenetic and slowed moments are established through formal and atmospheric juxtaposition in order to presence the mind and body. Furthermore, this thesis considers spatial and perceptual viscosity as a tool for exploring and representing temporal inhabitance. 

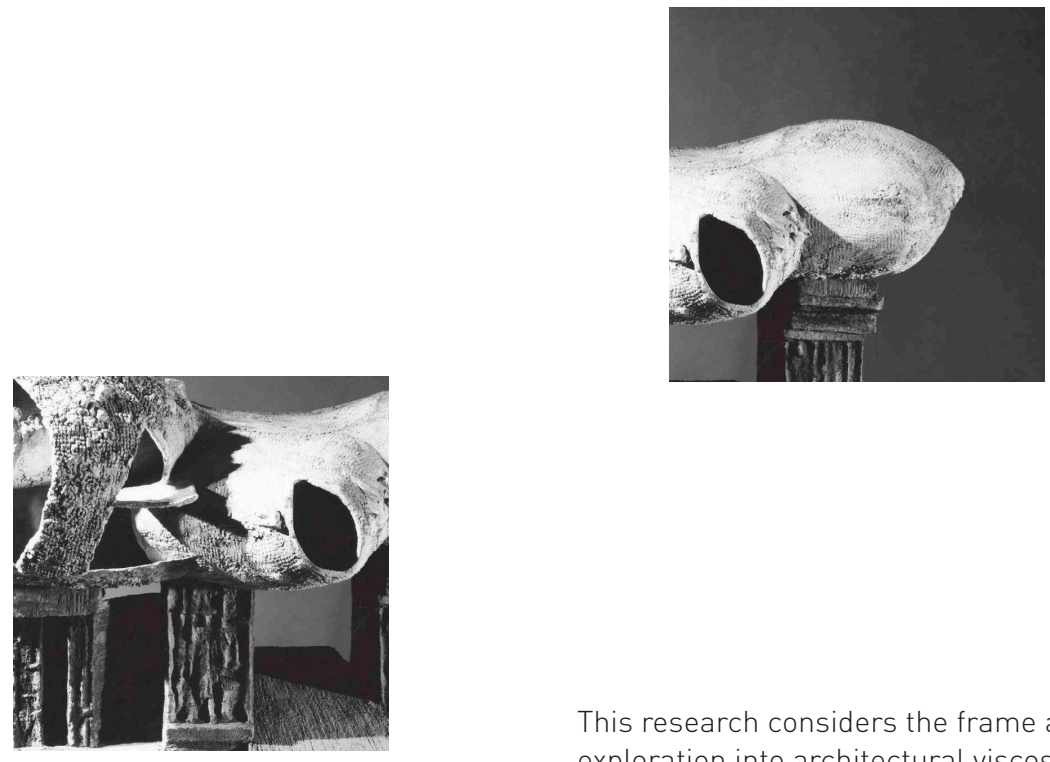

This research considers the frame as the mode of exploration into architectural viscosity as it represents a slice within the everyday sequence of events. Frame, in the context of this design research, is representative of memorable experiences that formulate moments of consciousness within the mind and body. In this regard, the frame holds all elements of space, from the atmospheric to formal, and what happens of and within them is pertinent in its response. This thesis uses the dwelling as a vehicle for this exploration; the enmeshed relationship between speed, time, and presence begins the layered procession that guides the inhabitant towards an architecture of slowness. 


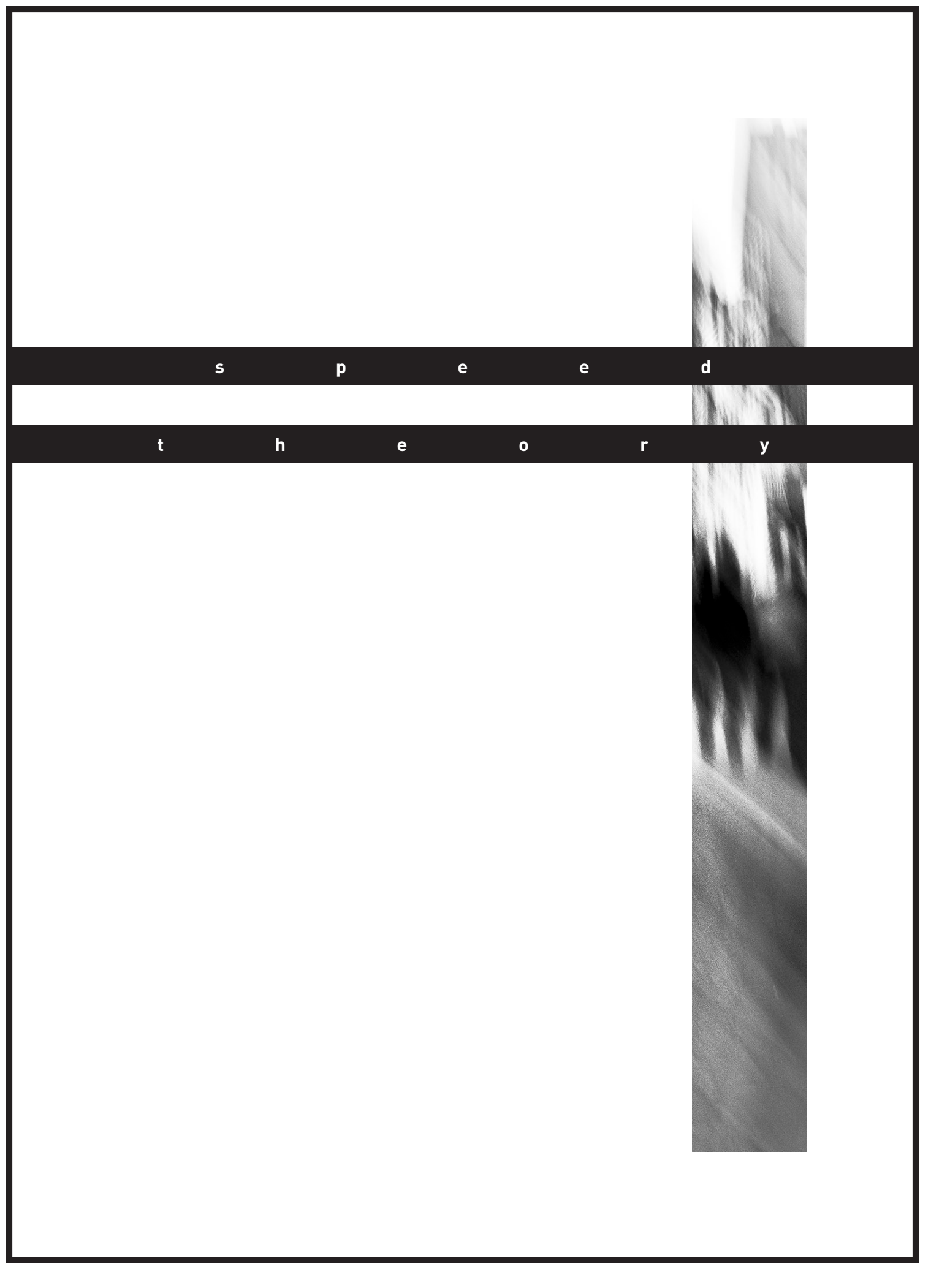


a modernity infused with such a sense of urgency that pace becomes the basis for establishing priority, defending property and patent claims, measuring productivity, progress, profit, intelligence, accomplishment, value, and pleasure.*

the acceleration of everything

This chapter explores the culture of speed and its effects on time.

f2

The Dark Side of The City 2015

André.G.

\section{1}

Science defines speed as the rate which a thing moves; it is considered high speed when it moves quickly and low speed when it moves slowly. The car is used as a contemporary example to define these speed variances, thus, If the car is travelling at a slow pace it is defined as low speed since it requires more time to reach its fixed destination; whereas, if it is travelling at an accelerated rate it is defined as high speed since it reaches its destination in less time. The everyday freneticism is not experienced in the way science defines speed, rather, it formulates through the amalgamation of moments, relative by nature, that flow and collide with one another to create the everyday procession. As such, the acceleration of certain components within the contemporary world has drastically affected the experience of the everyday, so much so, that individuals have begun to experience life in repetitive moments: wake up, drive, work, drive, sleep, and repeat. ${ }^{1}$ Ruled by schedules, time frames and speed, society suffers from the delusive acceptance of time as a divided experience. Today's accelerated culture has resulted in the unavoidable presence of speed taking over everyday life. In Milan Kundera's novel, Slowness, he attributes today's acceleration to technology and characterizes its effects on an individual:

Speed is the form of ecstasy the technical revolution has bestowed on man. As opposed to a motorcyclist, the runner is always present in his body, forever required to think about his blisters, his 


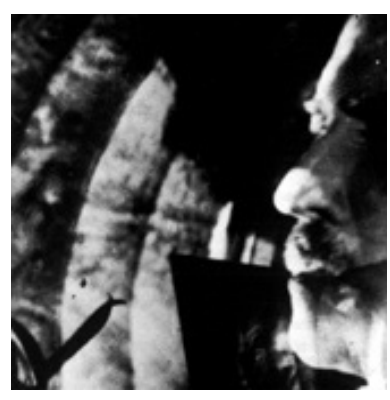

exhaustion; when he runs he feels his weight, his age, more conscious than ever of himself and of his time of life. This all changes when man delegates the faculty of speed to a machine: from then on, his own body is outside the process, and he gives over to a speed that is noncorporeal, nonmaterial, pure speed, speed itself, ecstasy speed. ${ }^{2}$

The origins of the contemporary fascination with speed can be traced back to Futurism, an avantgarde movement during the twentieth century. The Futurists celebrated the advances of technology and modernity by praising the machine, speed and change. ${ }^{3}$ The culture emerged from technology, and yet, it was the thrill of speed as pleasure that furthered the desire to quicken all aspects of life. ${ }^{4}$ Social historian, Theodore Zeldin, discusses how technology has "... been a rapid heartbeat, compressing housework, travel, entertainment, squeezing more and more into the allotted span. ${ }^{5}$ Instigated by the twentiethcentury advances in technology, the most notable influence was in transportation, this included trains, automobiles, and aircrafts. In the Manifesto of Futurism, F. T. Marinetti affirms, "that the world's magnificence has been enriched by a new beauty: the beauty of speed...Time and Space died yesterday. We already live in the absolute, because we have created eternal, omnipresent speed." ${ }^{\circ}$ Furthering this obsession with speed and its absorption of time, the two begin to associate with measure rather than experience and in doing so their relationship transitioned into one where speed and time equate to one another.

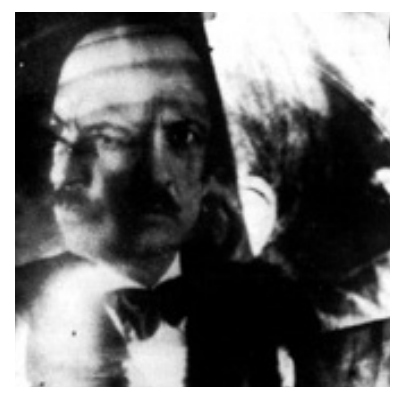

Futurist Portrait of Marinetti 

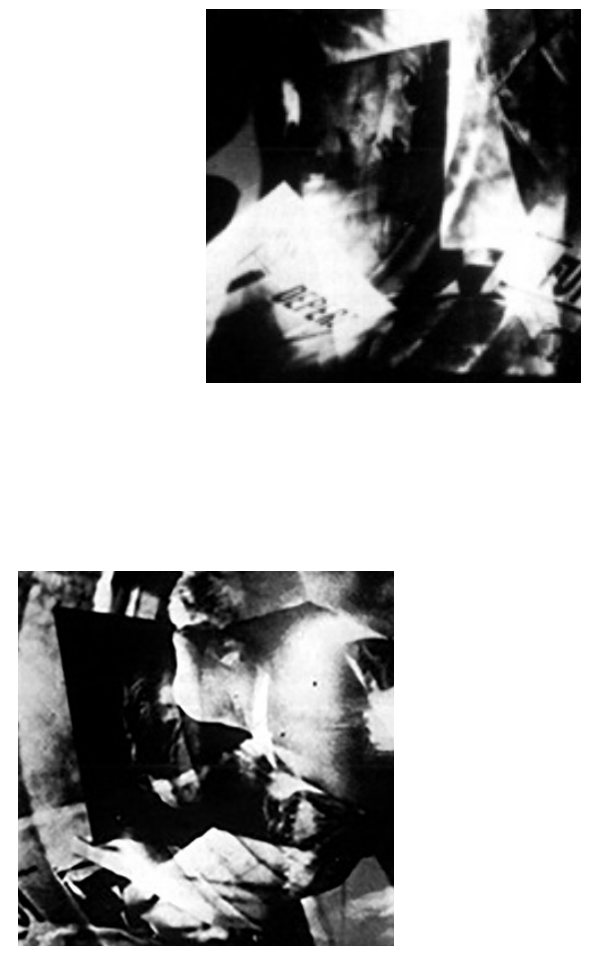

The association of speed and time was solidified by the invention of the clock, or rather the fascination with timekeeping. Through history, timekeeping has evolved from the study of astronomical bodies to the study of atoms. This increase in accuracy allowed individuals to measure the passage of time. In 1945, a method called atomic or molecular magnetic resonance was used by Physicist Isidor Isaac Rabi to suggest a clock based on the study of atoms and molecules. ${ }^{7}$ In 1949 the most accurate clock, known as the atomic clock, was developed. ${ }^{8}$ Its standardization, in the late 1960's, resulted in the acceptance of time as the measure of seconds. ${ }^{9}$ Writer and architectural theorist, Sanford Kwinter's Architectures of Time explores the historical evolution of time through science, literature, and drawing. Kwinter considers, "...the origin of the clock as the demise, rather than the invention of time." 10 He exclaims, "But the clock, we must remember, did not produce time, it merely standardized it and permitted, or rather forced, it to be correlated."11 The role of speed within modernity is twofold: both quickening, or frenetic speed, and slowing, or slowness. American anthropologist, Jeffery Schnapp, describes the relationship between frenetic speed and slowness as a dual emanation of a single system where the frenetic reigns. ${ }^{12}$ The fixation with frenetic speed stems from a sense of urgency through which pace is prioritized. ${ }^{13}$ In architecture, this urgency has been translated into the need for flexibility and efficiency. Within the last two centuries, our accelerated culture has required architecture to focus on pace rather than experience. This adoption of freneticism has resulted in an architecture which disassociates perceiver from perceived or subject from object. 


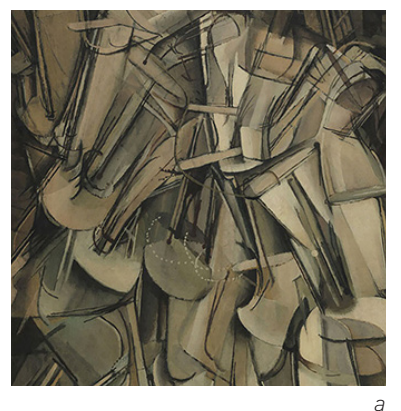

a

This thesis considers slowness as a tool to combat the contemporary fascination with frenetic speed. Through spatial and perceptual connectivity, an exploration emerged into the perception and reception of architectural frames or spaces. The contemporary distortion of the everyday is caused by spatial cuts wherein thin and insignificant experiences hinder the temporal qualities of inhabitance. Within these conditions, users receive their environment in a quickened blur, in turn, this accelerated rate of reception limits the ability to perceive in a viscous manner. In order to advance the conversation about architecture, a shift from momentary experiences of architectural spectacles to prolonged and viscous experiences of temporal architecture will bring consciousness to the forefront in order to presence the mind and body.

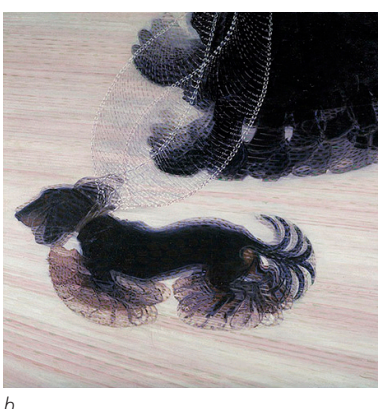

$b$

a_Nude Descending a Staircase

1912

Marcel Duchamp

b_Dynamism of a Dog on a Leash

1912

Giacomo Balla

Balla and Duchamp use repetition to depict the sequential experience of a thing; both conveying how movement is perceived. Balla's piece represents movement as the dynamic representation of speed whereas, Duchamp represents it with notions of quickening and slowing.

The last two centuries have created a culture of speed that has redefined the current notion of distraction into an experienced absentmindedness. As previously discussed, the culture emerged from technology, and yet, it was the thrill and pleasure of speed that furthered the desire to quicken all aspects of life. French cultural theorist and urbanist, Paul Virilio, is identified as the philosopher of speed for his robust work on the impacts of speed and technology on society; A Landscape of Events includes a series of essays that focus on these concepts and their effects on the pace of contemporary life. When discussing these implications on human social interaction, Virilio 


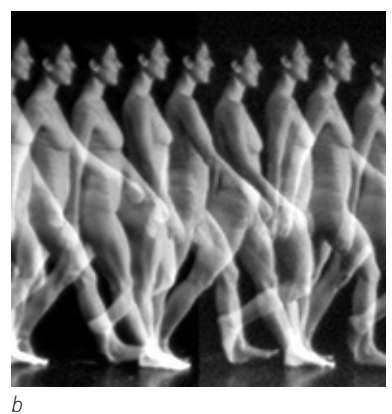

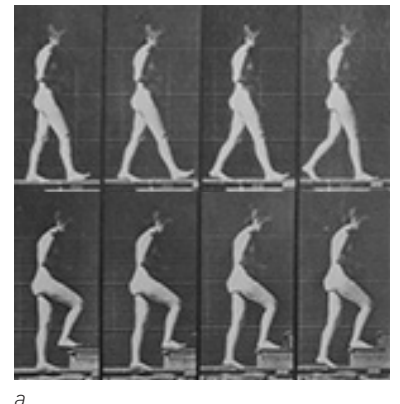

a

f5

a_Walking, Under Various Conditions

1881

Eadweard Muybridge

b_Chronophotograph of Walking Woman

1893

Étienne-Jules Marey

Balla and Duchamp were both inspired by the stop-motion photography of Étienne-Jules Marey and Eadweard Muybridge. The photographers used what is known today as multiple exposure techniques to analyze a series of moments in time within their photography. Their influence was seminal to understanding the implications of varying motions on the body. considers two centuries ago to be the beginning of an era "...that pushes us to desire lack or absence more than presence." 14 With the influx of media readily available on various portable devices in an the already absentminded society, contemporary speed is defined as an experienced unconsciousness whereby a layer of distraction has been built between the mind, body and everyday acts. This resultant layer is attributed to the increasing demands of modern life; Theravada monk, Nyanaponika Thera, discusses the mental and physical unconsciousness associated with these hectic demands, which, for Thera, make "it impractical to introduce such a slow-down of functions into the routine of the average working day. ${ }^{15}$ In this regard, the everyday is perceived in a perpetual state of distraction, which can be physical, cognitive, or both.

Within this absentmindedness our surroundings have become means of passage for the body, rarely contemplated backdrops to the experienced freneticism. ${ }^{16}$ In The Work of Art in the Age of its Mechanical Reproducibility, philosopher and cultural critic Walter Benjamin criticizes the reception of our surroundings, specifically architecture, which he considers to be received in a constant state of distraction. ${ }^{17}$ For Benjamin, individuals are made weary by the flood of visual stimuli within modern life; thus seeking refuge in mental distractions warrants an interchange between attraction and distraction within the everyday. ${ }^{18}$ However, the contemporary interplay between attraction and distraction or conscious and subconscious does not create Benjamin's "mental distractions", rather, stimuli is coated with more, but varying, stimuli that can be held in one's very hand (the smartphone). In this regard, Thera 


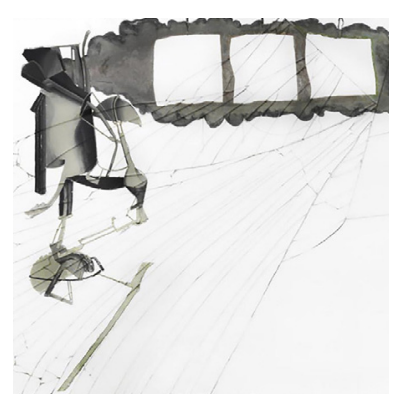

considers the antidote against the chaotic speed and absentmindedness of modern life to be in mindfulness, which is defined as the consciousness of one's internal and external experiences. ${ }^{19}$

Within architecture, spatial and perceptual density develops through the collision of contrasting moments, in turn, defining the contemporary interchange between frenetic and slow experiences. This experienced speed, relative by nature, requires attraction and distraction or conscious and subconscious moments of overlap to presence the mind and body by heightening the experience of slowing down. In this sense, without experiences of quickening there lacks slowness; in order to create an architecture of slowness, contrasting moments or thresholds of juxtaposition are used to vary levels of consciousness within a choreographed slowing that enables temporal inhabitation.

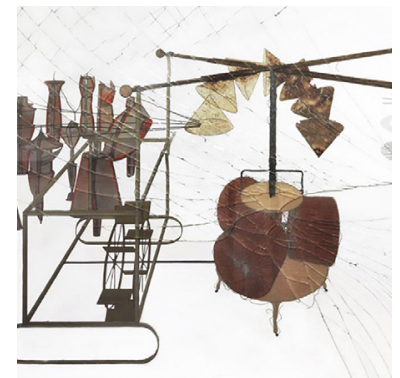

Reception is considered the act or process of receiving a thing; when associated with everyday experiences, this receiving is defined as the interplay between the perception and reception of the mind and body. Since the body is the vehicle through which space is experienced, the rate at which spaces unfold is due to the speed at which one moves. In this sense, ocular perception and speed are seen as inseparable entities..$^{20}$

Today's accelerated world privileges momentary architecture suited for hectic modern life; this presents a challenge for the discourse as people and architecture have lost their ability to inhabit time. Within modern society, physical distractions are associated with predetermined or monotonous 


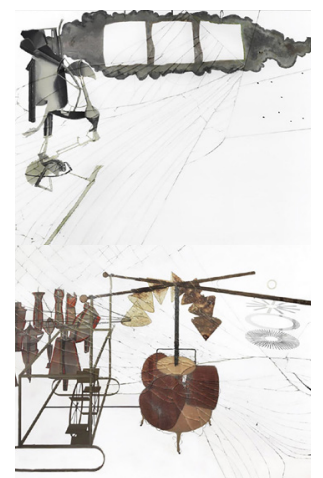

actual composition

\section{f6}

The Bride Stripped Bare by Her Bachelors, Even or Delay in Glass 1915-1923

Marcel Duchamp

Duchamp challenges normative drawing methods through a piece which uses glass as the canvas in order to question both painting and its conventions through delays. In this context, delay refers to the postponement of the pictorial becoming. In an architecture of slowness, postponement heightens the perception and reception of architectural spaces. courses, specifically habitual everyday act; whereas, cognitive distractions are the result of today's excessive visual stimuli. As a result, individuals have become disassociated from their experiences. American author and historian, James Gleick, uses the motion of a racquetball to describe this relationship: “...our speed is as much in how we see as how we move. When a racquetball hits a wall there is a moment-less than a millisecond-when the leading and trailing edges of the sphere go inside out and meet in the center, so that the ball resembles a doughnut... Only in an age of speed could we stop time. Only in an age of speed would we need to." ${ }^{21}$ The racquetball's speed limits the perception and reception of its actual movement, therefore, what is seen is a mere blur of its motion towards and contact with a wall. ${ }^{22}$ Modern life is like the racquetball, experiences are perceived in an obscure fragmented state between location, A, and destination, B. As previously discussed, due to the everyday freneticism this fragmentation neglects corporeality for efficiency. This neglect correlates with modern distraction; for Virilio, only through conscious perception, or apperception, can a dialogue form between perceiver and perceived or subject and object:

"If speed then serves to see, to conceivethat is, to seize reality, and not just to get around-this is because it is part and parcel of perceptual faith, that ocular belief that is inseparable from our immediate awareness...the more readily you accede to the scenes that unfold before you, the more consistency you give your existence. Your existence suddenly becomes commutative with 


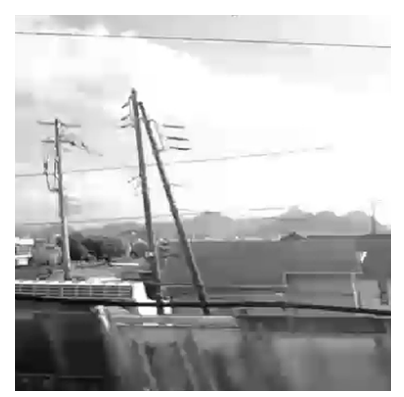

an environment to which you lend credence." 23

As explained above, individuals are distracted by both pace and task, therefore, redefining modern reception involves slowing down the mind and body in order to activate an interchange between movement and perception. In Slowness, Kundera, identifies this relationship: "the degree of slowness is directly proportional to the intensity of memory [and] the degree of speed is directly proportional to the intensity of forgetting." 24 In this regard, the relationship between speed and movement is pivotal in creating apperceptive, or fully conscious, experiences.

By slowing down, one heightens awareness and limits fragmentation by increasing attachments and continuity within the everyday. Finnish architect and author, Juhani Pallasmaa, considers "the experience of enveloping spatiality, interiority and hapticity [as] the deliberate suppression of sharp focused vision." 25 For Pallasmaa, focused vision establishes distance between the subject and object when centering on a specific task, whereas, unfocused vision embeds the individual within their environment when heightening the peripheral layers of experience. ${ }^{26}$ Both Virilio and Pallasmaa argue for a more conscious everyday experience. Therefore, by slowing sensorial experiences of architecture, we heighten temporal inhabitation in order to create a mindful physical presence.
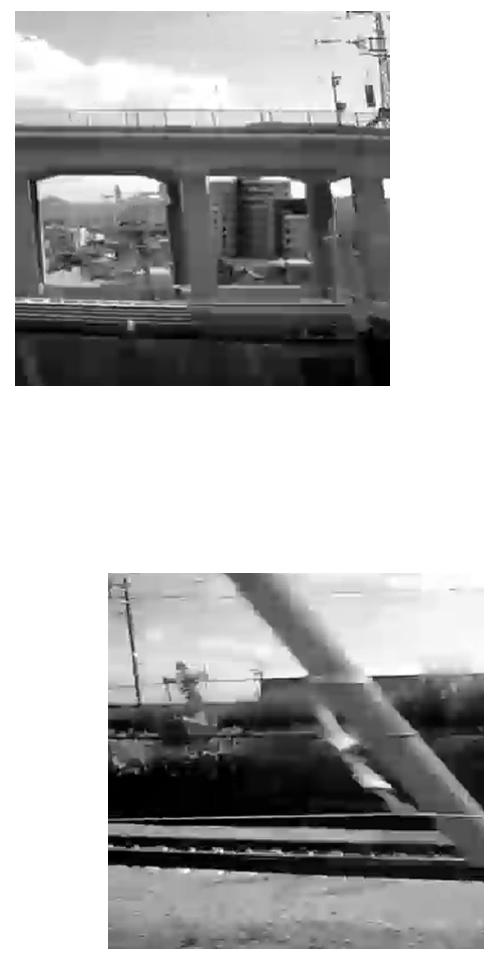

Stills from the Bullet Train; Japan

2016

Sahel Tahvildari

These stills were taken from a bullet train travelling from Osaka to Tokyo. The maximum operating speed of a bullet train is approximately $320 \mathrm{~km} / \mathrm{h}(200 \mathrm{mph})$; as a result, objects that are typically perpendicular to the ground are seen as stretched. 


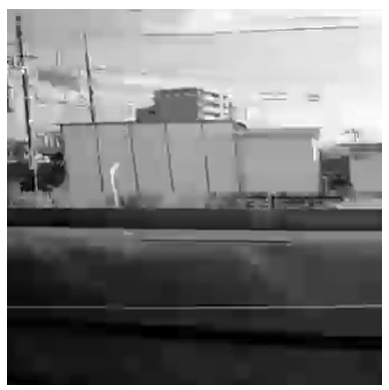

\section{montage as stills $\mathbf{1}$.3.1}
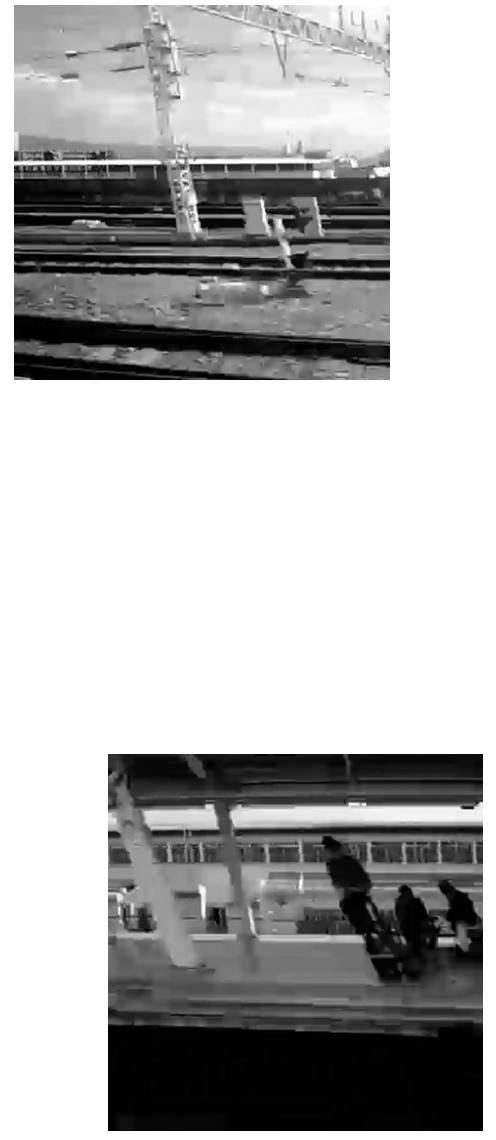

The everyday pace is both the cause and effect of today's primacy of speed; as such, the reception of space has become a thin and distorted representation of one's temporal and spatial inhabitance. The question lies in how the speed of movement - slowing down, speeding up, reversing, and pausing - affects an inhabitant's spatial and perceptual receptors. The design experiment, Montage as Stills, asked a friend to walk through the Max Gluskin House by Hariri Pontarini Architects, for the first time in three varying speeds: accelerated, slowed, and with moments of pause. The walk included ramps, stairs, corridors, rooms, and spatial transitions, in turn, allowing research into the relationship between speed and the sequential nature of one's spatial and temporal experiences; characterized as an unfolded experience. This research considers the normative experience of space to analyze the impact that acceleration has on presencing the mind and body.

Within this design research, presence plays a pivotal role in creating a dialogue between mind, body, and space. The experiment considers how, and if, slowing an individual's experience creates a more viscous architecture which inhabits time through slowness.

Theoretically, it is assumed that the physical aspects will be emphasized within the accelerated walk, while the emotive and sensorial aspects will be emphasized with the slowed and paused walk. Thus, slowing down and eventually pausing will most likely render the friend able to increase their reading of the experience; in turn, thickening the passage of space and time. 
The expectations defined above came to fruition with some unanticipated but fascinating results, they include:

For the accelerated walk, the friend reported on the physical aspects of the experience. However, an unpredicted outcome was their awareness of the social aspects within the space. They described their accelerated walk with limited use of first person pronouns, as if the speed in which they moved detached them from the experience. In the first walk, the most prominent use of first person pronouns was within the description of their body's exertion while ascending the stairs.

For the slowed walk, the friend reported on the sensorial aspects of the space. By slowing down, they began to identify the relationship between themselves and the architecture while using first person pronouns to describe their entire experience. This newfound attachment between subject and object is defined as place attachment. Place attachment is an emotional bond between person and place or perceiver and perceived. Evidently, as the body slows down, the connection with a particular place or moment increases.
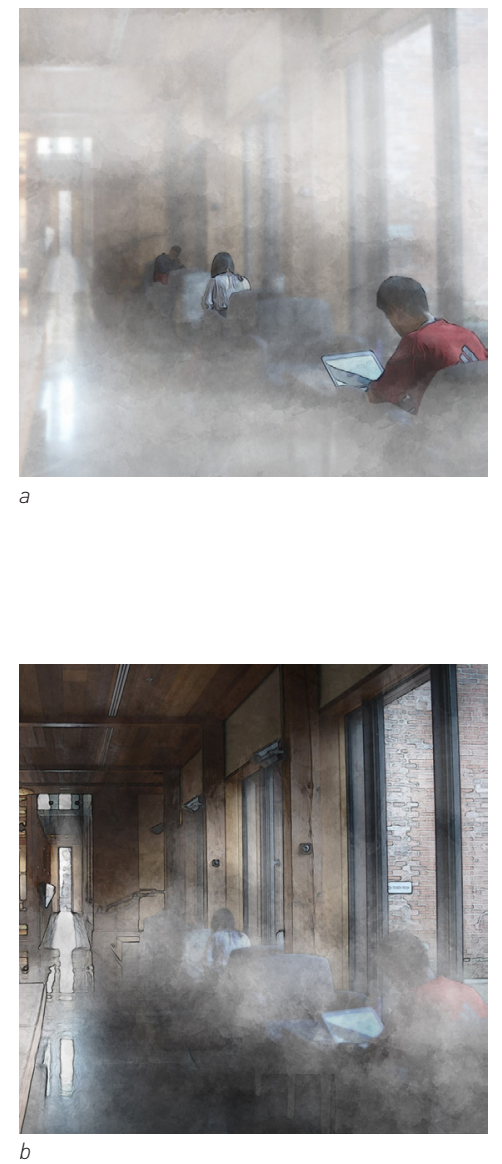


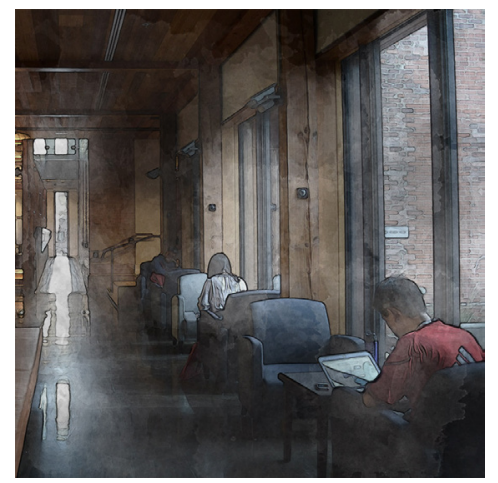

t8

Speed Perception

a_Accelerated Walk

b_Slowed Walk

c_Paused Walk

Speed Perception demonstrates what is perceived at varying speeds. With the accelerated walk, focus is placed on the social aspects within space. With the slowed walk, focus is placed on the sensorial aspects within space. With the paused walk. focus is placed both on the social and sensorial aspects within space.
For the paused walk, the friend reported on the increased sensorial aspects of the space. Similar to the accelerated walk, an unpredicted outcome resulted from a layering of their awareness of both the social and sensorial aspects of the space. The friend began to further their attachment with both the place and the people occupying it. By allowing moments of pause, they could overlap the first experience, social and physical, with the second experience, sensorial and cognitive.

In conclusion, as the friend encountered, there are two forms of presence: physical and cognitive. The first experiment revealed an exhaustive presence, one which contributes to speed and exertion. The second experiment revealed a sensorial presence, one which supports slowness and place attachment. By slowing down, the perceiver comes into a dialogue with the perceived; a dialogue which forms both attachments and memories with a particular place or moment. The contemporary relationship between individuals and architecture relies on passivity in which experiences are thin, insignificant, and momentary. As cognition is brought to the forefront, the mind and body is slowed within an architecture of spatial and temporal inhabitance: an architecture of slowness. 


\section{attempt $\mathbf{0 1}$ \\ accelerated}

The first attempt, referred to as the accelerated walk, asked the friend to walk swiftly through the building and to describe their experience. As previously noted, the friend will likely report on the physical aspects of the experience, while placing emphasis on the act of moving.

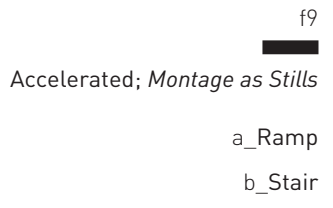

By emulating the direction oriented experience of frenetic speed, Montage as Stills, highlights how acceleration distorts the perception and reception of space. 


\section{"It was pretty fluid. The connections between the old and the new aren't really noticed when you walk through. It} actually seems like parts of it that were newer, now that I'm looking at it from the outside, seem old inside. But there were moments that were really nice; a lot of it had to do with the skylights, which would always draw my attention up really quick. But also, the materiality changed, bringing certain materials that are exterior into the interior. Especially on the ground level, walking through the corridor and looking out through the courtyard, being able to see the glass through to the entrance at the front. It was the layering of all those things that were really impactful."

Did you notice your body's physicality or the architecture because of the speed in which you were moving?

What I honestly noticed more was the social aspects of the space. So I noticed the people in the space more than the actual space.
"Where I needed to get to. When I was walking up the stairs I thought about how tired I was. So, at that moment I felt my body, in the sense that I was tired or I was sore. But I kept thinking. 'I need to get up these stairs, because I need to go here. 


\section{attempt $\mathbf{0 2}$ \\ slowed}

The second attempt, referred to as the slowed walk, asked the friend to repeat the same walk through the building slowly and to describe their experience. As previously noted, the friend will likely report on the emotive and sensorial aspects of the experience, while placing emphasis on the perception of space.

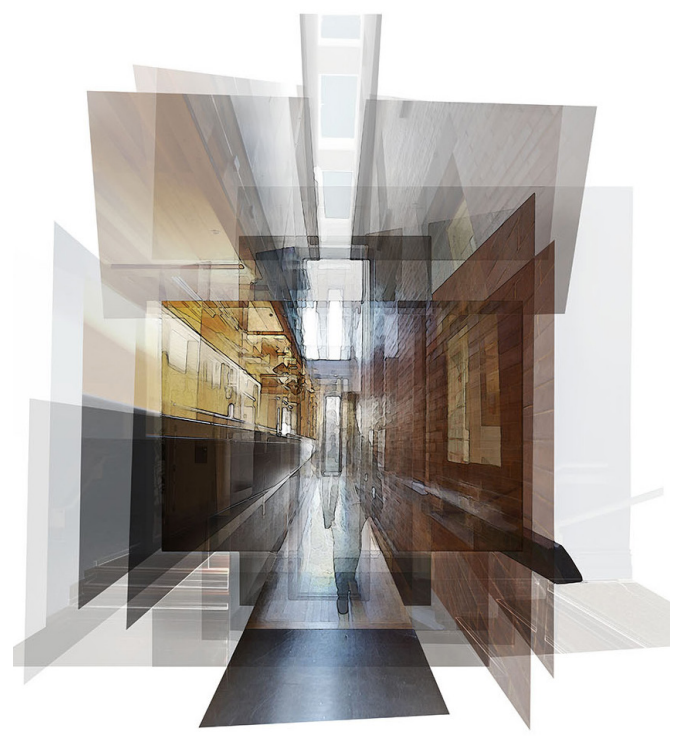

Slowed; Montage as Stills

$$
\begin{gathered}
\text { a_Ramp } \\
\text { b_Stair }
\end{gathered}
$$

By emulating the viscous experience of slowness, Montage as Stills, highlights how slowing down thickens the perception and reception of space.

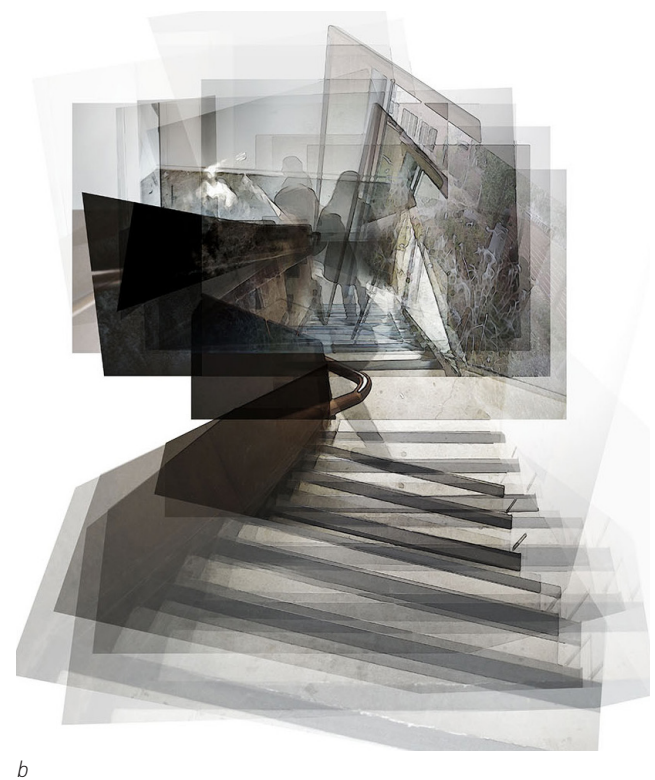


"Thinking back to it, I realized how much I missed because I was moving so fast. "

Please elaborate. but also the ramp up. The light coming in and the window in the back framed the moment beautifully."

What would you say was the main difference by slowing down?

\begin{tabular}{l} 
"I actually noticed what was around \\
me. Physically. In my first walk I noticed \\
people and just small hints of things, but \\
\hline by going through it slowly I thought back \\
\hline on certain things that I didn't remember \\
seeing the first time because I was \\
moving so quickly. I saw them this time \\
because I was able to see it, because I \\
gave myself the time to see it."
\end{tabular}

As well the skylights, especially the one in the stairwell, was really impactful. As was moving up the stairs, I kept getting different views of it and it was almost like I was tripping over myself because I just wanted to take it in. However, the hallway on the second floor is still quite mundane; when looking at it, it clearly wasn't done well, it was as if they needed a hallway so they just made a hallway. Coming down that final stair, the shadows cast by the window made a beautiful impression which moved because of the wind outside. Then coming back around and turning the corner to see the student lounge again, 


\section{attempt $\mathbf{0 3}$ \\ paused}

The second attempt, referred to as the paused walk, asked the friend to repeat the same walk through the building with the addition of three to five moments of pause and to describe their experience. As previously noted, the friend will likely report on the emotive and sensorial aspects of the experience, while placing emphasis on the layers of perception, including spatial and social.
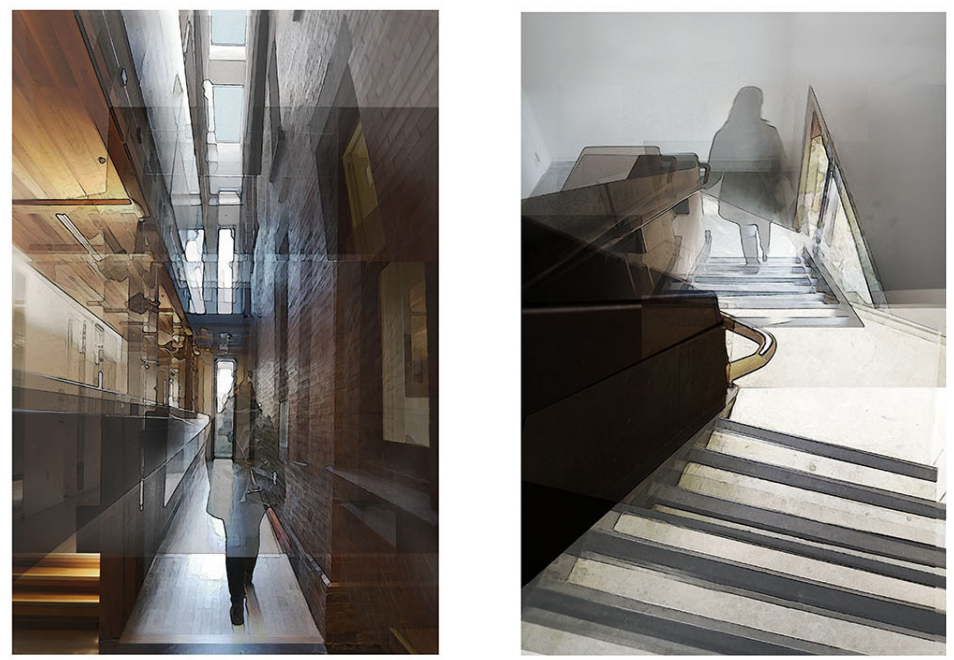

a

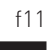

Paused; Montage as Stills

$$
\begin{gathered}
\text { a_Ramp } \\
\text { b_Stair }
\end{gathered}
$$

By emulating the deepened experience of space within moments of pause or rest, Montage as Stills, highlights how pausing subtracts movement to increase presence through concentration and awareness. 
Describe your experience.

\section{"What was interesting about having the moments of pause was the ability to overlap my first experience, which was fast, with my second experience, which was slow. What I mean is that in my slow experience I noticed the architecture and the spaces, but in my fast experience I noticed the social aspect of the spaces. In being able to pause I was able to overlap the two. So, not only did I notice the space and its atmosphere, but I also saw how other people were interacting in that space. I thought that was really interesting."}

"My first moment of pause was on the ramp, looking up into the skylight. What was the most impactful, in that space. was the contrast created by the lighting. My second moment of pause was in the staircase, about two-thirds of the way up, where the skylight was framed by the darkness underneath the stairs. What was impactful here was the way the corten steel and the white of the skylight highlighted the blue of the sky. My third moment of pause was in the

\begin{tabular}{l} 
second staircase as I was going down, \\
\hline where a speckled shadow was created \\
on the white wall by whatever was on the \\
\hline window. Seeing the wood grain of the \\
\hline handrail and how that contrasted with the \\
corten created a really beautiful moment. \\
My fourth moment of pause would be \\
looking back towards the ramp, through \\
the lounge space, when I realized that \\
either side of the lounge was glazed to \\
an exterior space. I had noticed it before \\
but, on the one side, the trees kind of sit \\
behind people as if they are connected \\
with the space, while on the other side is \\
the courtyard. My fifth moment of pause \\
would be just as I stepped out into the \\
courtyard, when I looked up and saw the \\
contrast of the corten steel with the blue \\
sky."
\end{tabular}

Were there parts of the experience that you hadn't noticed in the first two experiences? Such as the stairs.

\begin{tabular}{l} 
"I think the detailing of the stairs. How it \\
actually came together, if there were gaps \\
in certain areas. Even the texture, I was \\
\hline able to stop and feel certain things that I \\
wasn't necessarily able to feel before."
\end{tabular}


Notes

1 James Gleick, Faster: The Acceleration of Just About Everything (Toronto: Vintage Books, 1999), 11.

2 Milan Kundera, Slowness (New York: Harper Perennial, 1997), 2.

3 Lawrence Rainey, Christine Poggi, and Laura Wittman, eds. Futurism: Anthology (New Haven: Yale University Press, 2009), 1.

4 Enda Duffy, The Speed Handbook: Velocity, Pleasure, Modernism (Durham: Duke University Press, 2009) 18.

5 Gleick, Faster: The Acceleration of Just About Everything, 11.

6 F. T. Marinetti, "The Founding and Manifesto of Futurism" in Futurism: An Anthology, ed. Lawrence Rainey, Christine Poggi, and Laura Wittman (New Haven: Yale University Press, 2009), 51.

7 Claude Audoin and Bernard Guinot, The Measurement of Time: Time, Frequency, and the Atomic Clock (Cambridge: Cambridge University Press, 2001), 52-53.

8 Ibid., 53.

9 lbid., 59.

10 Sanford Kwinter, Architectures of Time (Cambridge: The MIT Press, 2001), 21.

11 Ibid.

12 Jeffrey Schnapp, "Fast (Slow) Motion” in Speed Limits, ed. Jeffrey Schnapp (Milan: Skira, 2009), 27. 
13 Ibid.

14 Paul Virilio, A Landscape of Events, trans. Julie Rose (Cambridge: The MIT Press, 2000), 61.

15 Nyanaponika Thera, The Power of Mindfulness (San Francisco: Unity Press, 1972), 37.

16 Anthony Vidler, Warped Space: Art, Architecture, and Anxiety in Modern Culture (Cambridge: The MIT Press, 2000), 79.

17 Ibid.

18 Ibid., 82.

19 Thera, The Power of Mindfulness, 37.

20 Virilio, A Landscape of Events, 40.

21 Gleick, Faster: The Acceleration of Just About Everything, 63.

22 Ibid.

23 Virilio, A Landscape of Events, 40.

24 Kundera, Slowness, 39.

25 Juhani Pallasmaa, The Eyes of the Skin: Architecture and the Senses (London: John Wiley \& Sons Ltd., 2005), 10.

26 Ibid. 


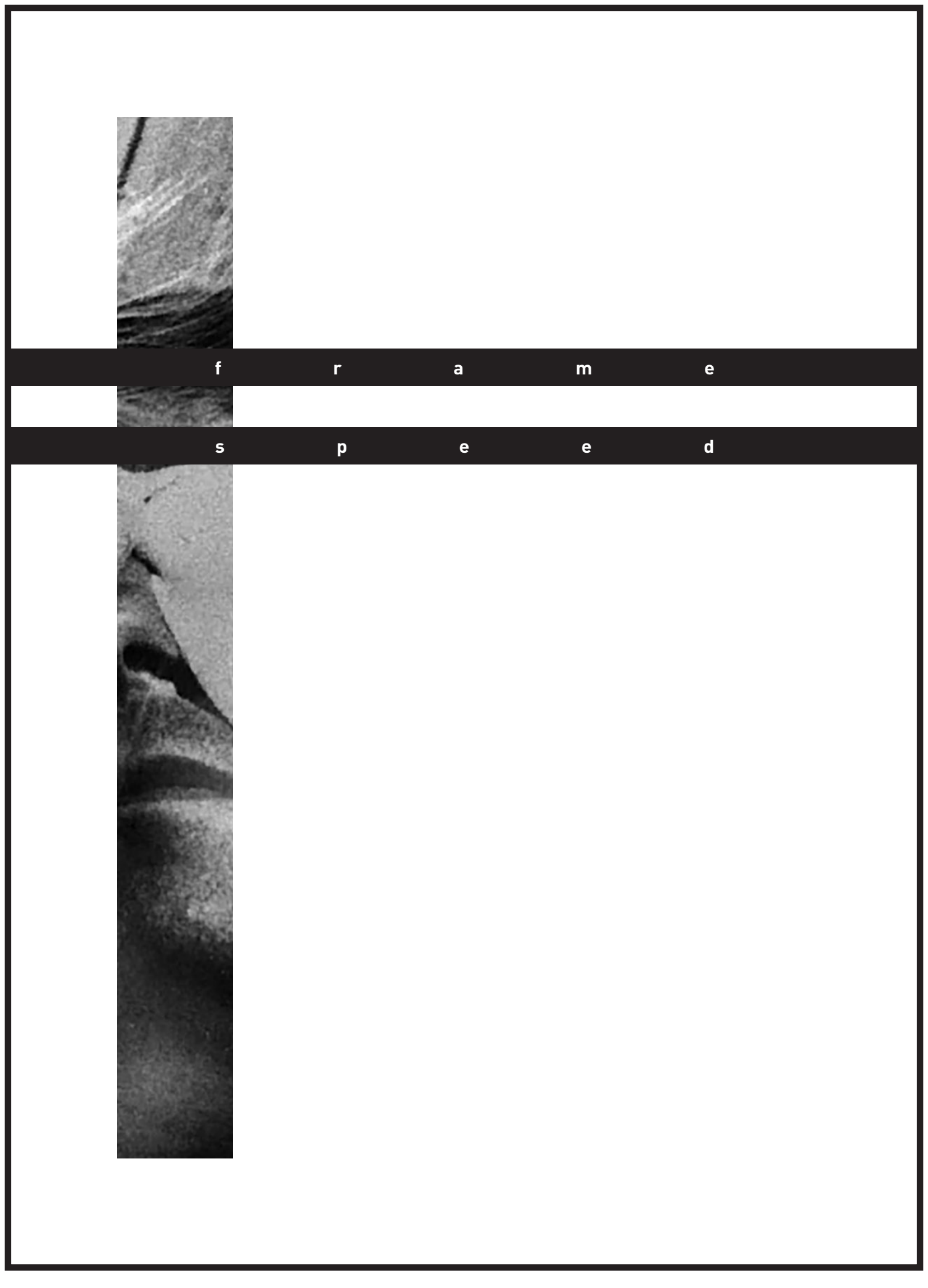


Architecture exists, like cinema, in the dimension of time and movement. One conceives and reads a building in terms of sequences. To erect a building is to predict and seek effects of contrast and linkage through which one passes."

cinema and time

This chapter explores time and its implementation in film. f12

La Jetée Still 1962

Chris Marker

\section{1}

Cinema uses two complementary elements to organize slices or images of everyday events to produce the illusion of continuity. ${ }^{1}$ These elements, embedded within the assembly of a film, include time and motion; both of which, are powerful components that create emotive effects within a cinematic unfolding. By existing within time, cinema attempts to capture and emphasize temporal progression as qualities of time are present in all aspects of filmic frames.

Cinematography developed quickly from the early photographic experiments led by Eadweard Muybridge and Étienne-Jules Marey whereby consecutive frames were used to record the movement of a subject. The rate at which consecutive images are displayed to create a moving picture is defined as the frame rate or frames per second (FPS). Although films typically use a uniform frame rate, its increase or decrease can create a more blurred or fragmented reading. Dissolving, cutting, and fading are techniques used to control the sequence and movement of and within film; these methods are manipulated in various ways to represent the progression of time. ${ }^{2}$ Soviet avant-garde filmmakers, Sergei Eisenstein and Andrei Tarkovsky, differ in their methods to harnessing temporality within film. Eisenstein considers the temporal aspects of film to be captured in the sequential nature of its frames; whereas, Tarkovsky considers the assembly of frames to further the pressure of time, as he believes both time and movement to be present within each frame. ${ }^{3}$ In this sense, cinematic time can be experienced in both 


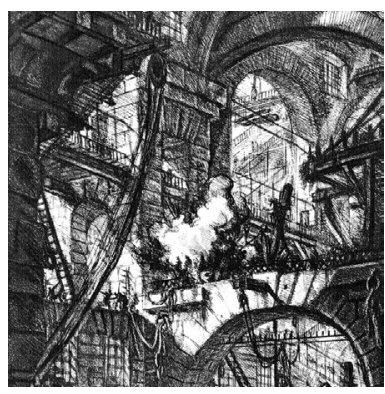

the sequential representation of its frames and the movement that occurs within them.

Similarly, architecture is experienced as the summation of and within its parts; its elements are used to create moments of juxtaposition within a spatial and perceptual unfolding. This form of unfolding is prevalent in the work of Giovanni Battista Piranesi, an Italian artist famous for his etchings of Rome, who used immense detailing and evocative lighting to create images of atmospheric prisons. Similar to filmic frames, the density within the etchings moves the eye around the image. This density provoked Eisenstein's analysis of Piranesi's work, where he found that an explosion of the image put the elements into a metaphorical motion that lead one piece to the next. ${ }^{4}$ Eisenstein describes the work as the recreation of architectural fantasies, where "some planes, opening up to infinity behind each other, carry the eye into unknown depths, and the staircases, ledge by ledge, extend to the heavens, or in a reverse cascade of these same ledges, rush downward." 5 This rhythmic unfolding is a dynamic construct where the inhabitant plays a pivotal role in representing the numerous trajectories present in space. ${ }^{6}$

In this sense, the reading of architectural space "does not depend merely on a single frame... but on a succession of frames or spaces."7 The assembly of space establishes moments or thresholds of juxtaposition, which create emotive gaps within an architectural experience. For Tschumi, these gaps - corridors, doors, staircases, and ramps - become a space of their own, inserted between each event

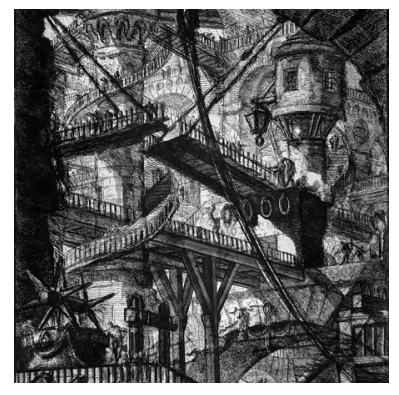

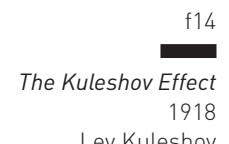

a_Soup equals Hunger

b_Child at Rest equals Sadness

c_Woman equals Lust

The same blank face is juxtaposed with a secondary frame to prompt an emotional response. 

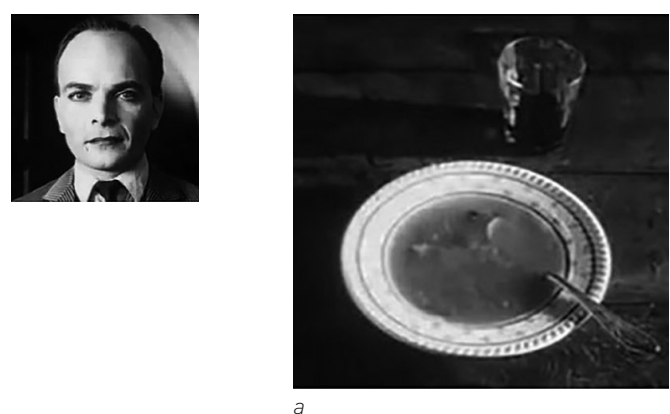

in an expanded sequence. ${ }^{8}$ The sequential nature of architecture draws explicit parallels with the experience and composition of film. Through a montagist approach, whereby frame sequencing is pertinent, contrasting moments of frenetic and slowed inhabitation are developed through spatial and perceptual collision.

cinematic montage $\mathbf{2 . 2}$
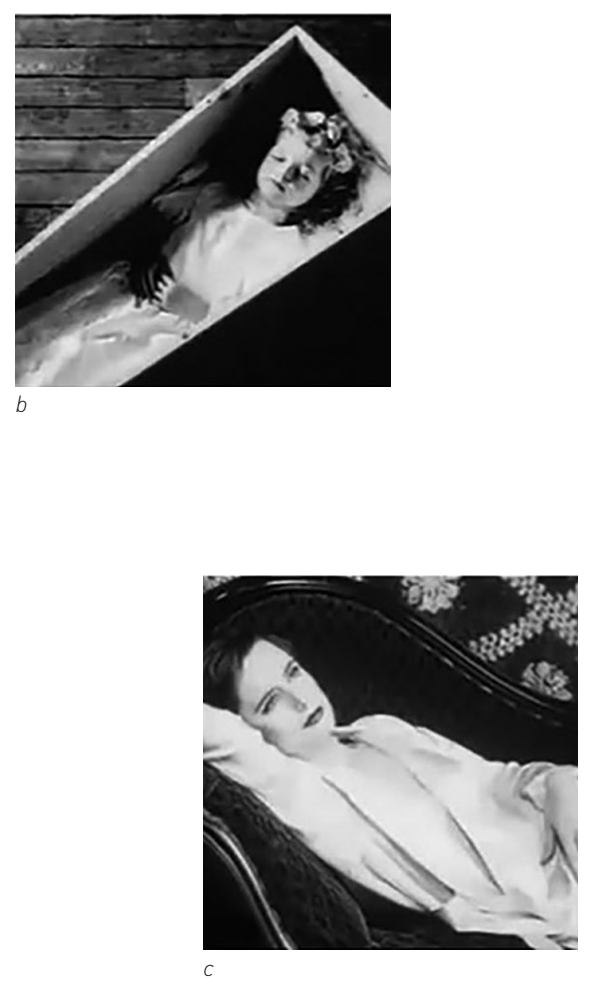

Montage is a method whereby juxtaposing or superimposing images are combined into a single composition. Within cinematography, montage is an editing technique that juxtaposes various frames to compress space and time. This editing technique was formulated in the early twentieth century by Russian film practitioner and theoretician, Lev Kuleshov, who conducted a renowned experiment where a single image was crosscut with another to give the impression of concurrent actions. ${ }^{9}$ This powerful cinematic technique, formally known as the Kuleshov Effect, juxtaposes two unrelated frames to give rise to a new frame, which became an emotive interpretation of the proceeding frames.

After its conception, the technique was adopted in various ways by French, American, British and Soviet film practitioners. It was most influential to the works of Soviet avant-garde filmmakers, including Eisenstein, due to its distinct reliance on the active engagement between the viewer and the subject. ${ }^{10}$ French filmmakers merely considered montage to be the process of editing or assembling a film; whereas, American and British filmmakers assembled the 

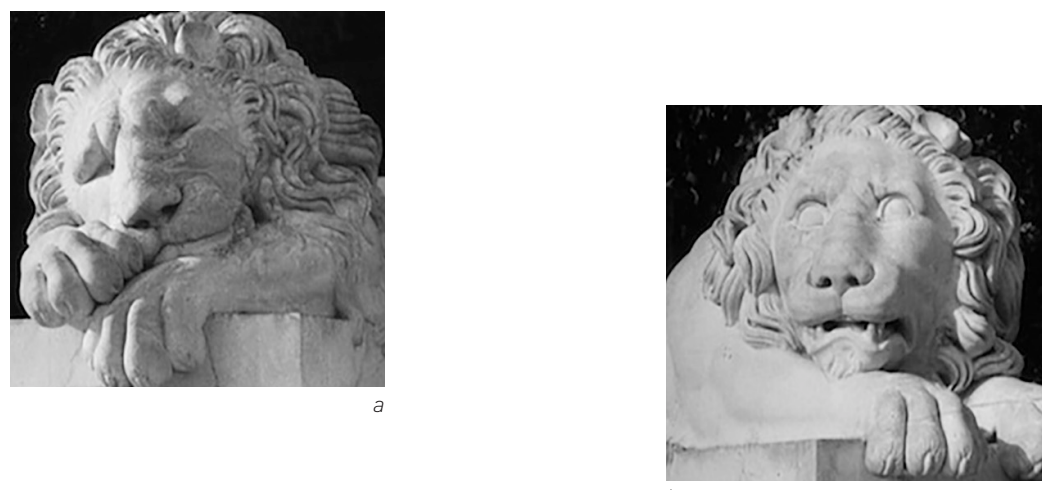

most important shots to condense space, time, and narrative. The most comprehensive study into the emotive response associated with cutting and editing was predominantly found in Soviet filmmaking. In Sculpting in Time, Tarkovsky explores the implications of time on space, whereby, "the distinctive time running through the shots makes the rhythm of the picture... [one that is not determined] by the length of the edited pieces, but by the pressure of time that runs through them." "11 For Tarkovsky, this pressure is influenced by the atmospheric qualities present in the frame. These elements include light, shadow, material, scale, and emotion, which reappear as prominent components in the creation of an architecture of slowness. Although the montage style of Eisenstein and Tarkovsky vary in several ways, both experiment with juxtaposition to create an emotive response within the subject.

This emotive response is an embodied reaction to an experience. In The Work of Art in the Age of its Mechanical Reproducibility Walter Benjamin suggests that both film and architecture are communicated through the tactile realm, unlike painting which is purely visual. ${ }^{12}$ Benjamin uses the assembly of filmic frames through the metaphor of the magician and surgeon to explore the tactility of film; "The magician maintains the natural distance between himself and the patient...the surgeon does the opposite: he reduces the distance to the patent a great deal (by actually going inside him)...Magician and surgeon behave like painter and cameraman. The painter, while working, observes a natural distance from the subject; the cameraman, on the other hand, penetrates deep into the subject's

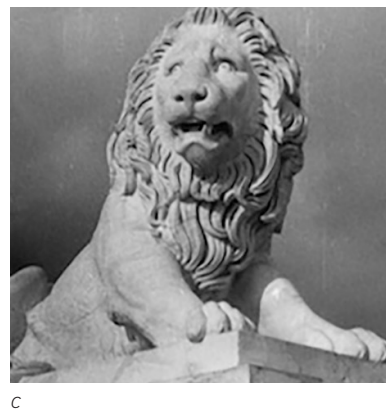

Battleship Potemkin 1925

Sergei Eisenstein

a_Sleeping Lion

b_Awake Lion

c Risen Lion

In Battleship Potemkin, Eisenstein cuts from shots after the battleship bombs Odessa to the statue of a sleeping lion, a lion that is awake, and a lion that has risen. This frame sequence is meant to represent the rising of people at the onset of a revolution. In film. the collision of frames are used as a technique to further the narrative while instilling an emotive response within the viewer. 


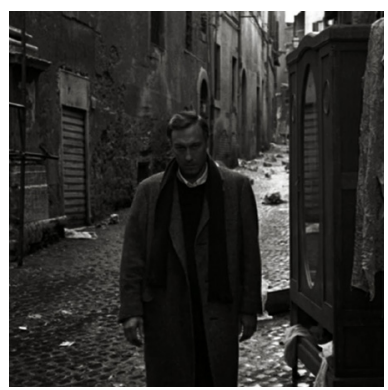

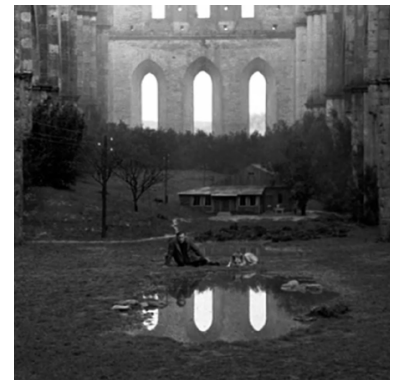

f16

Nostalghia

1983

Andrei Tarkovsky

Tarkovsky's Nostalghia uses time, space, and memory to represent the internal and immeasurable passage of time. Through the rhythmic unfolding of moments in space and time the sequential nature of everyday life is explored. tissue." ${ }^{13}$ Unlike art, film is a kinesthetic experience that penetrates deep into the subject, which presences the entire body. Similarly, architectural space is experienced kinesthetically where both embodied and haptic images translate into the memory. ${ }^{14}$ This bodily experience was explored by Eisenstein in Montage of Attractions, where he devised a narrative to stimulate the sensorial condition of the viewer; he used space and time as the "...discursive function of cinema and its potential to reorganize reality," one which is generated from the eye through to the mind and body of the viewer. This cinematic unfolding is representative in architecture, whereby the body, specifically the legs, construct meaning while moving along a path; for Eisenstein, this creates "a montage sequence for an architectural ensemble...subtly composed, shot by shot." ${ }^{15}$ The sequential nature of both architecture and film is experienced or read as they are traversed; this allows for the subject to settle within them as they settle within the subject's mind and body. ${ }^{16}$

The density and speed of and within architectural and filmic frames results in the quickening and slowing of time. The sequential nature of both architecture and film establishes a layered sequence which allows for these various speeds of reception and interaction within an experience. Through moments of spatial and perceptual collision, a tumbling through space and time traverses the mind and body between varying levels of stimuli. As previously discussed, the everyday freneticism has resulted in distracted experiences; however, similar to film, a thickening of architectural and filmic frames allows for more 
concentrated experiences. In The Work of Art in the Age of its Mechanical Reproducibility, Benjamin explores the differences between distraction and concentration, while highlighting the elements that increase concentration within filmic frames:

By close-ups of the things around us, by focusing on hidden details of familiar objects, by exploring commonplace milieus under the ingenious guidance of the camera, the film, on the one hand, extends our comprehension of the necessities which rule our lives; on the other hand, it manages to assure us of an immense and unexpected field of action. ${ }^{17}$

In this sense, an abundance of contrasting visual stimuli results in a felt quickening, whereas, limiting visual stimuli creates a felt slowing by increasing concentration. The following four films, from various directors and time periods, were analyzed to further explore the implications of visual stimuli to temporal inhabitance.

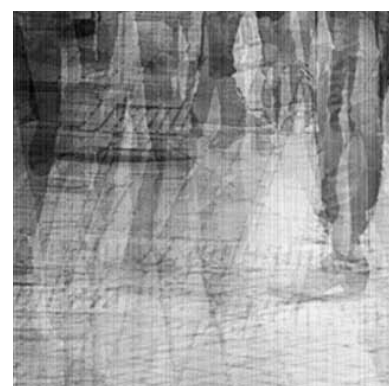



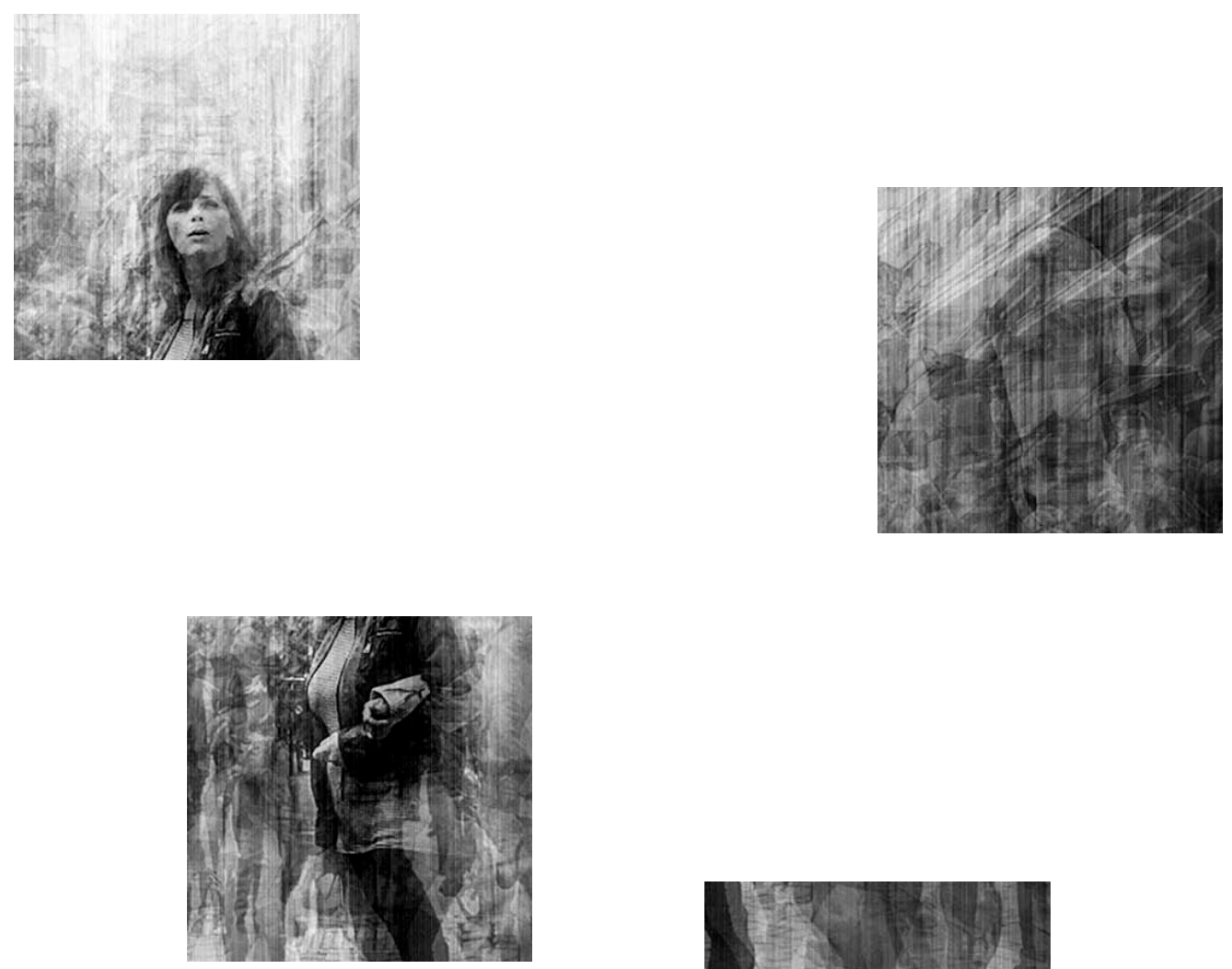

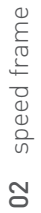

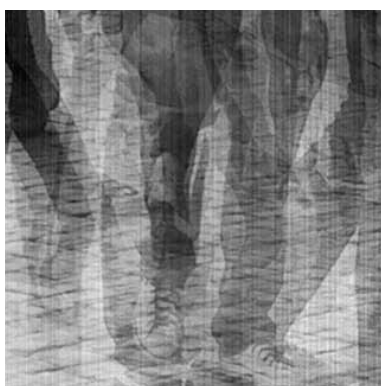




\subsection{1}

\section{Battleship Potemkin}

\section{Sergei Eisenstein}

1925
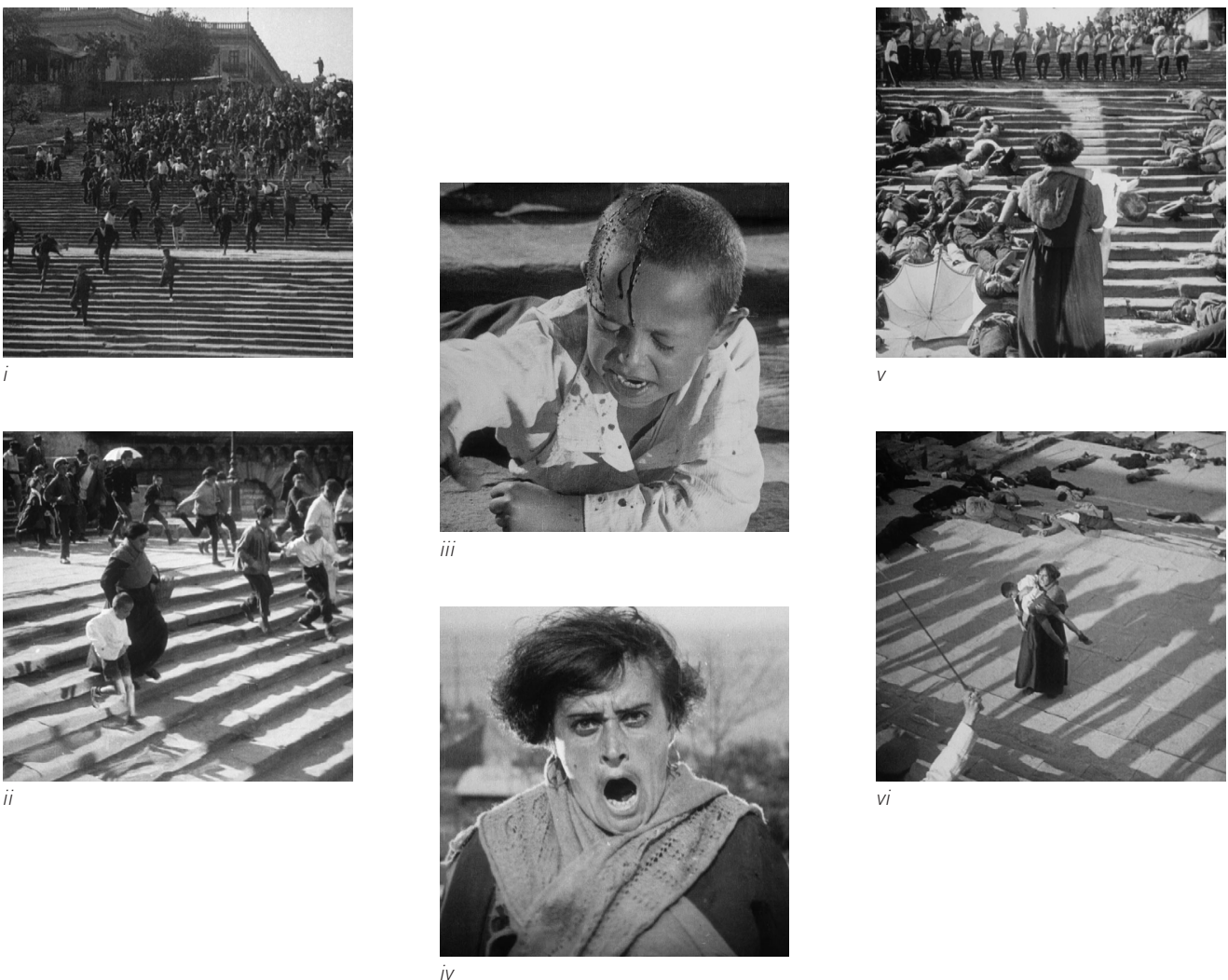
The silent propaganda film, Battleship Potemkin, depicts the 1905 rebellion which took place on the Russian Navy's Black Sea Fleet; due to the harsh conditions on board the sailors unite against the commanding officers. The film is split into five parts, it begins with the sailors refusing to eat soup made with rotten meat, which leads to the quarrel that kills sailor Vakulinchuk. After laying his body on shore for the people of Odessa to view, the angered citizens march in protest but are violently subdued by the military.

The most influential aspect of Battleship Potemkin is the assemblage of the film; Eisenstein uses the rapid juxtaposition of close-up or very close-up and wide shots to manipulate the emotive response of the viewer. Unique camera angles and perspectives take the viewer directly into the action occurring within a frame. As previously discussed, Eisenstein believes film to be a tactile experience which is read as it is traversed; he uses both the eyes and the legs to guide the mindful body through an intense narrative. Along with the film's frame variation, the addition of a dramatic score furthers the varying emotive qualities instilled within the viewer.
A prominent example of the film's montage technique is seen during the massacre on the steps of Odessa; Eisenstein uses one of the five types of montage, tonal, to elicit a reaction from the viewer. The sequence begins with an upbeat score as the people of Odessa wave goodbye to the departing sailors. Suddenly the people begin running down the steps and the score becomes frantic. Eisenstein uses an abundance of visual stimuli that cut between shots, they include varying perspectives of the soldiers and the people. A gruesome moment during the struggle is of a young boy running down the steps with his mother. As the boy is shot and falls down, the film cuts between his lifeless body being trampled by others and his mother's screaming face. The effectiveness of the alternating between perspectives immerses the viewer into the film while playing with the fluidity of space and time. 


\section{2 .2}

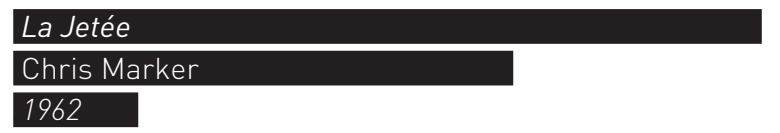

Chris Marker

1962
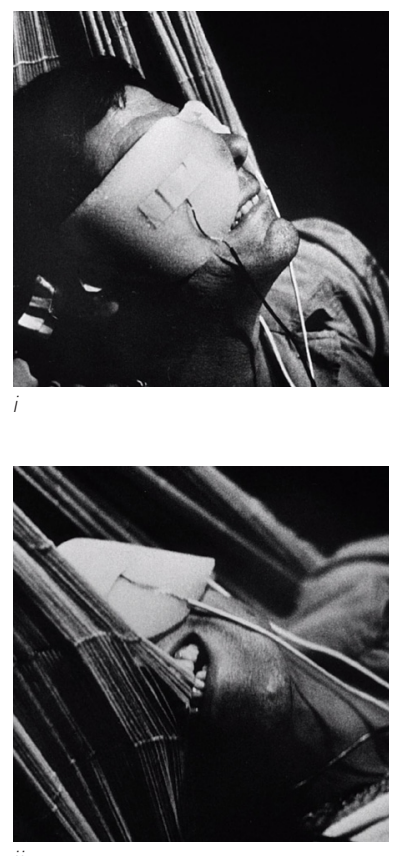
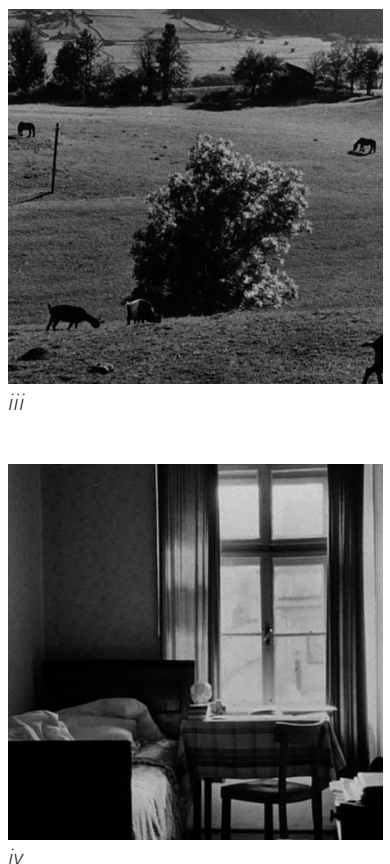

Stills: La Jeté

1962

Chris Marker
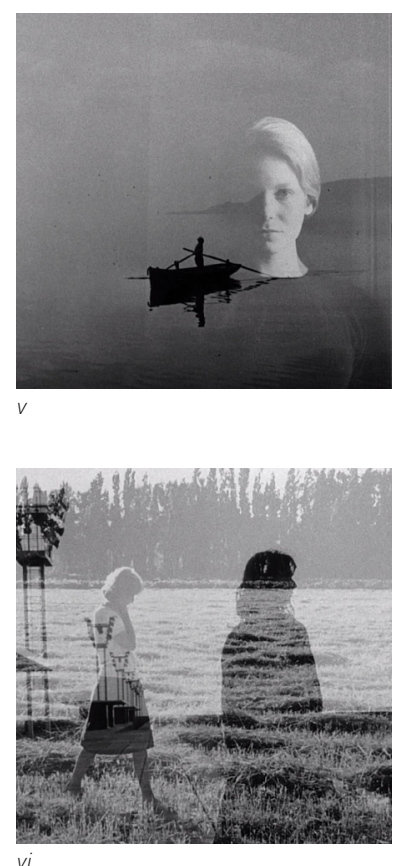
La Jetée uses still images to narrate the post-apocalyptic story of an unnamed Man chosen as the subject for time travel experiments due to his attachment with a traumatic memory from his childhood. The memory in question takes place at the main pier of Paris International Airport and includes a Woman's face and a Man's death. The film explores the dimensions of the human psyche through concepts of time, memory, and perception. Its nonlinear sequencing plunges the Man, along with the viewer, into the past, present, and future, and yet, by associating characters with levels of temporality, the film creates a coherent overlapping of time.

The most influential aspect of La Jetée is the assemblage of the film; Marker developed a photomontage technique that used still images, rather than moving images, to narrate the Man's story. By varying the pace of each image, editing and adding narration and sound effects allowed the film to achieve a sense of movement and time-lapse, pivotal to its continuity. The film alternates between cuts and dissolves to emulate the passage of time through a perceived quickening and slowing of events. As such, human perception suppresses the makeup of the film while becoming unaware of the cuts and transitions.
A prominent example of the film's remarkable assemblage is seen during the first couple of time travel experiments. Marker applied quick cuts of the Man's distressed face, at approximately 2-second intervals, while travelling into the past to emulate his pain and suffering. After which, a collection of several dissolves defines the Man's entrance and dispersal throughout time until he is introduced and seeks out the Woman. The effectiveness of alternating between cut, dissolves into darkness, and dissolves into different moments of time distinctly express the varying levels of temporal passage: from the quick cuts to the slowed dissolves. 


\section{2 .3}

\section{Mirror}

Andrei Tarkovsky

1975

1975
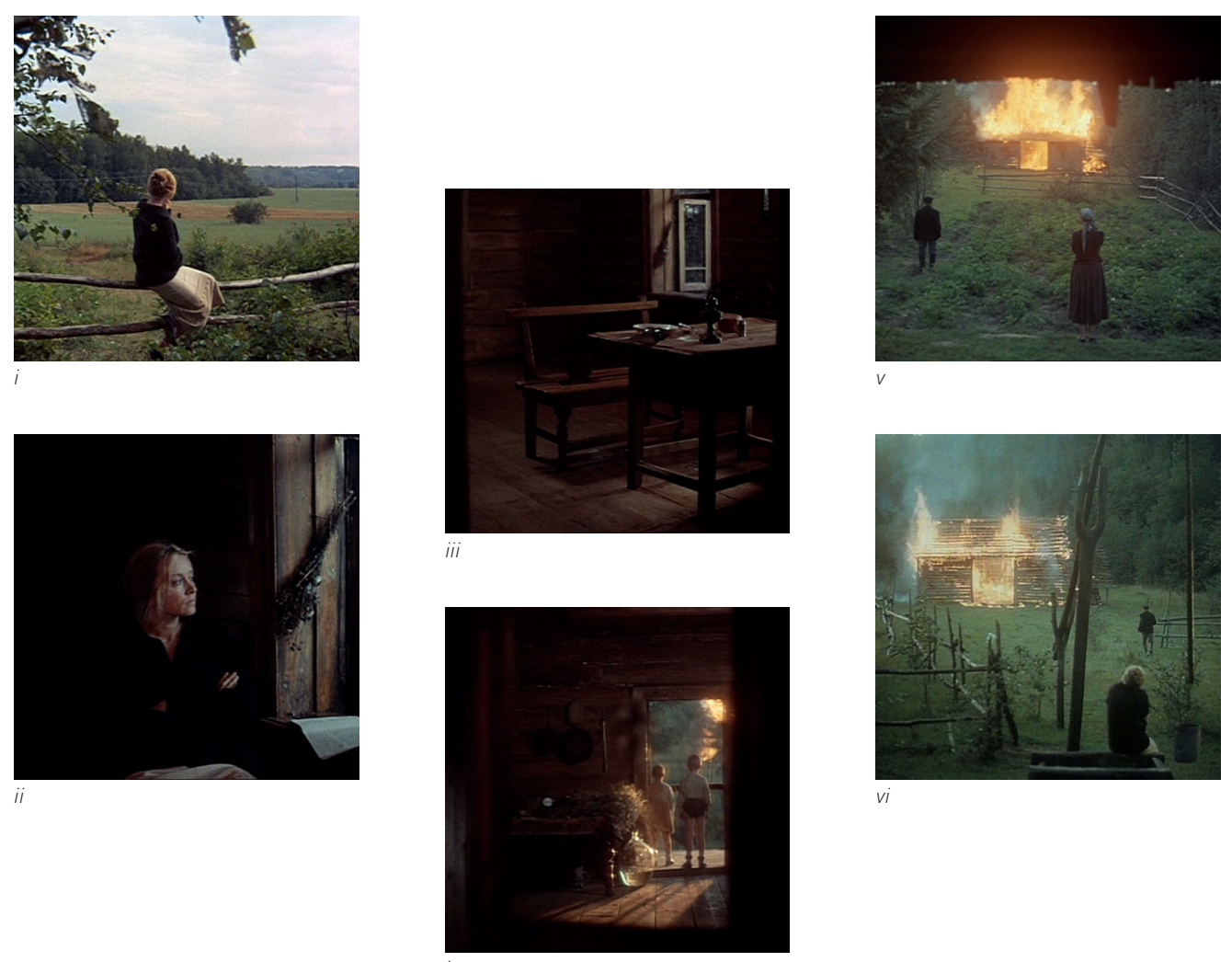
The autobiographical film, Mirror, depicts the introspective journey through the life of the narrator, Alexei, who plays surrogate for Tarkovsky himself. Similar to Marker's La Jetée, Tarkovsky intends to create a dialogue between the varying levels of time by immersing Alexei and the viewer into a complex temporal zone nestled between the past and present. Its nonlinear sequencing overlaps multiple elements within the film to reference the metaphoric mirroring of temporality while exploring the effects of time, memory, and war on the human psyche.

Both Marker and Tarkovsky's narrative move forwards and backwards in time, and yet, unlike La Jetée the considerable number of narratives told within Mirror plunges the viewer into pure confusion. This is due to the distorted reading of time which takes place through the perception, recollection, and memory of approximately four main characters. As previously discussed, Tarkovsky believes the internal rhythm of and within frames to be the core of temporal cinema. In this case, the film uses several continuous shots, often referred to as sequence shots, to emulate the rhythmic progression of time at varying moments in the characters' lives; cuts and dissolves alternate between both character and temporal changes within the film.

A prominent example of the film's slow rhythmic unfolding is evident with the burning barn sequence shot, whereby, the lack of cuts and focus on insignificant actions creates the felt capture and release of time pressure. During the sequence, an emphasis is placed on moving the viewer through space. It beings with two children running from the table to the porch to watch the burning barn as the camera lingers on the table until a bottle rolls off. After which, the frame pans to the left and in the reflection of a mirror the children are seen standing in the doorway. Various minor actions occur until the viewer is walked outside to watch the burning barn in deep focus, a cinematic technique with great depth of field, which furthers the felt slowness like the dissolves used in Marker's La Jetée. 


\section{2 .4}

Taste of Cherry

Abbas Kiarostami

1997
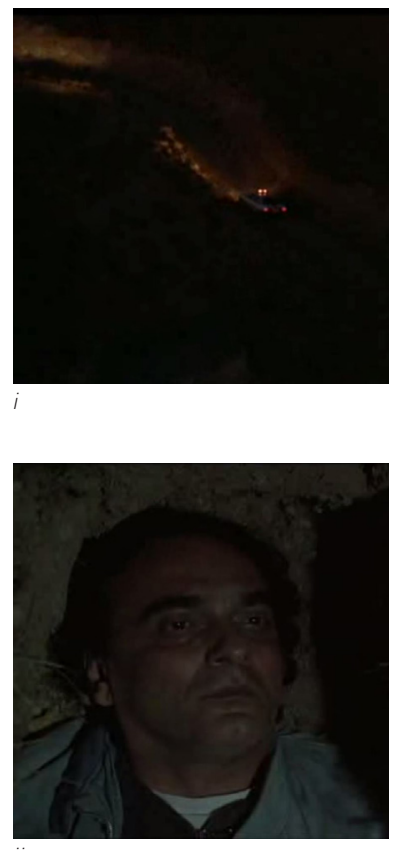

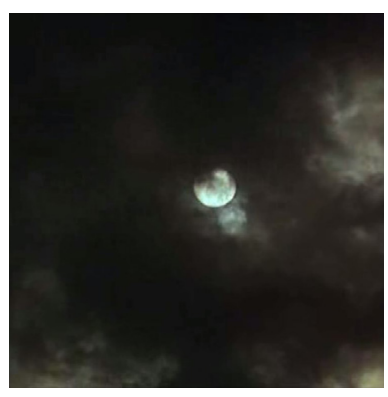

Stills: Taste of Cherry

1997

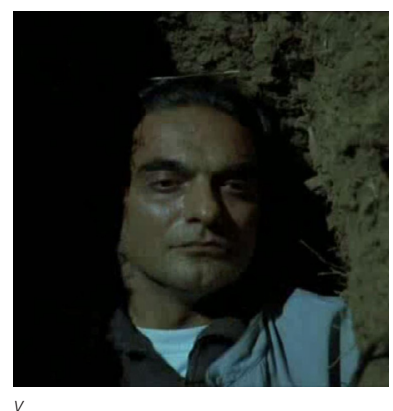

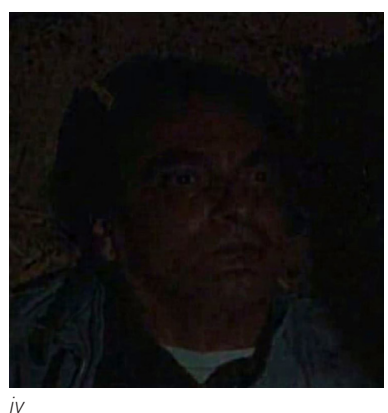

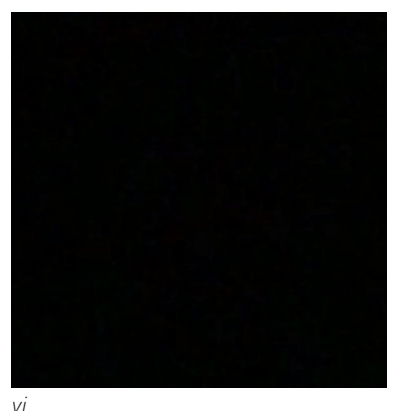


Taste of Cherry follows a middle-class Iranian man, Mr. Badii, as he drives around the hills of northern Tehran, Iran looking to hire someone, preferably a working-class labourer, to help him commit suicide. Both Taste of Cherry and Mirror are categorized as slow films under the Slow movement, which originated in Italy; such films are often minimal, observational, and emphasize contemplation with techniques that both extend and expand time. The film cuts prolonged medium close-up shots of lengthy conversations between Badii and or one of his four given passengers with extreme long-shots of the surrounding bare landscape along with the car meandering through it.

The most influential aspect of Taste of Cherry is the simplicity to its sensorial depth; Kiarostami overlaps atmospheric frames with various sounds, car horns, giggling children, dogs barking and squealing, and construction vehicles, to conceptually move the viewer beyond the frame. Unlike Eisenstein who thrives on the freneticism of juxtaposition, Kiarostami's projection into the world beyond the screen allows for moments of pause and contemplation in the filmic frame of the mind and body. The film's inherent minimalistic emptiness allows the viewer to be present, both mentally and physically, in the experience.

A prominent example of the film's depth is seen when Badii finally rests in his hole after taking an abundance of sleeping pills. During this extended sequence, the focus is placed on Badii and the Earth around him; the camera cuts from him, in the hole, to the clouds and the full moon above. As the clouds wash over the moon darkness befalls both Badii and the viewer, leaving them in complete emptiness. The sound of an oncoming thunderstorm becomes the focus as lightening repeatedly flashes Badii into and out of view until he is completely unseen. In the absence of light, Taste of Cherry allows the viewers awareness to transpose the film experience to themselves by introducing a moment of pause and contemplation. 

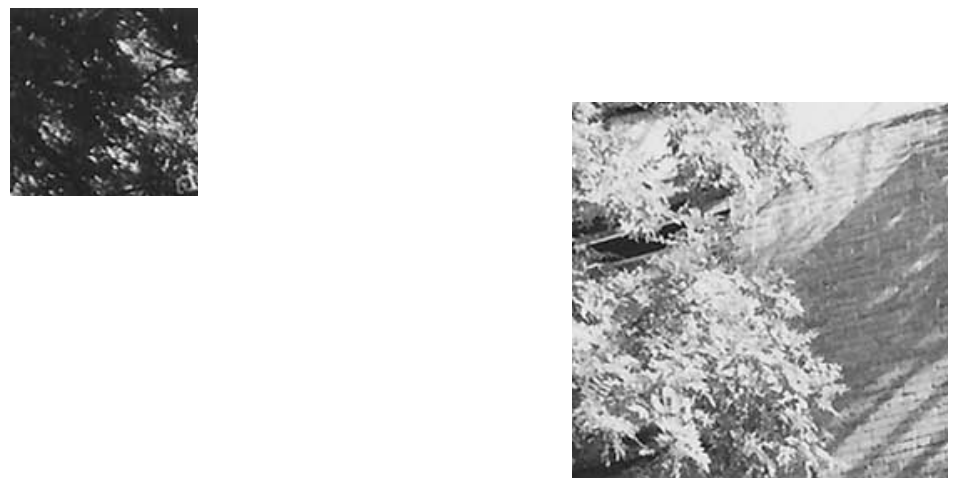

The above film analyses clarify the various implications filmic sequences have on a viewer's perception and reception. The range takes the reader from Sergei Eisenstein to Abbas Kiarostami in order to explore the differences, including technique and sequencing, between speed cinema and slow cinema. It was discovered that with speed cinema, cut sequences condense space and time through temporal juxtaposition, while slow cinema uses sequence shots to elongate space and time by moving the viewer slowly through the frame. From this, it is understood that the technique used of and within frames is pivotal to its interpretation. Within architecture, the juxtaposition of and within spaces creates moments of quickening and slowing within temporal inhabitance.

Like film, the everyday is perceived by way of touch; it consists of distinct actions or habits that unfold subconsciously as the eyes and body traverse through space. ${ }^{18}$ Habits are considered routine behaviours or acts that repeat regularly; "...the ability to master certain tasks in a state of distraction... proves that their performance has become habitual." 19 They reveal themselves frame by frame while embedding select moments of consciousness into the memory. For French Marxist philosopher and sociologist, Henri Lefebvre, habitual acts are "situated at the intersection of two modes of repetition: the cyclical, which dominates in nature, and the linear, which dominates in

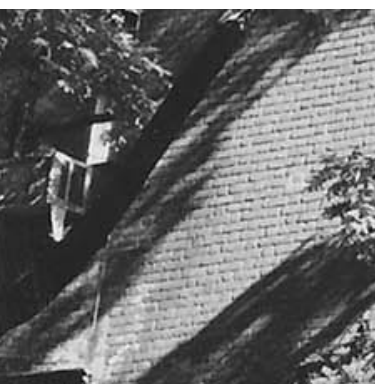
processes known as 'rational.'"20 Lefebvre explains the cyclical to be the natural repetition of nights and days,

.



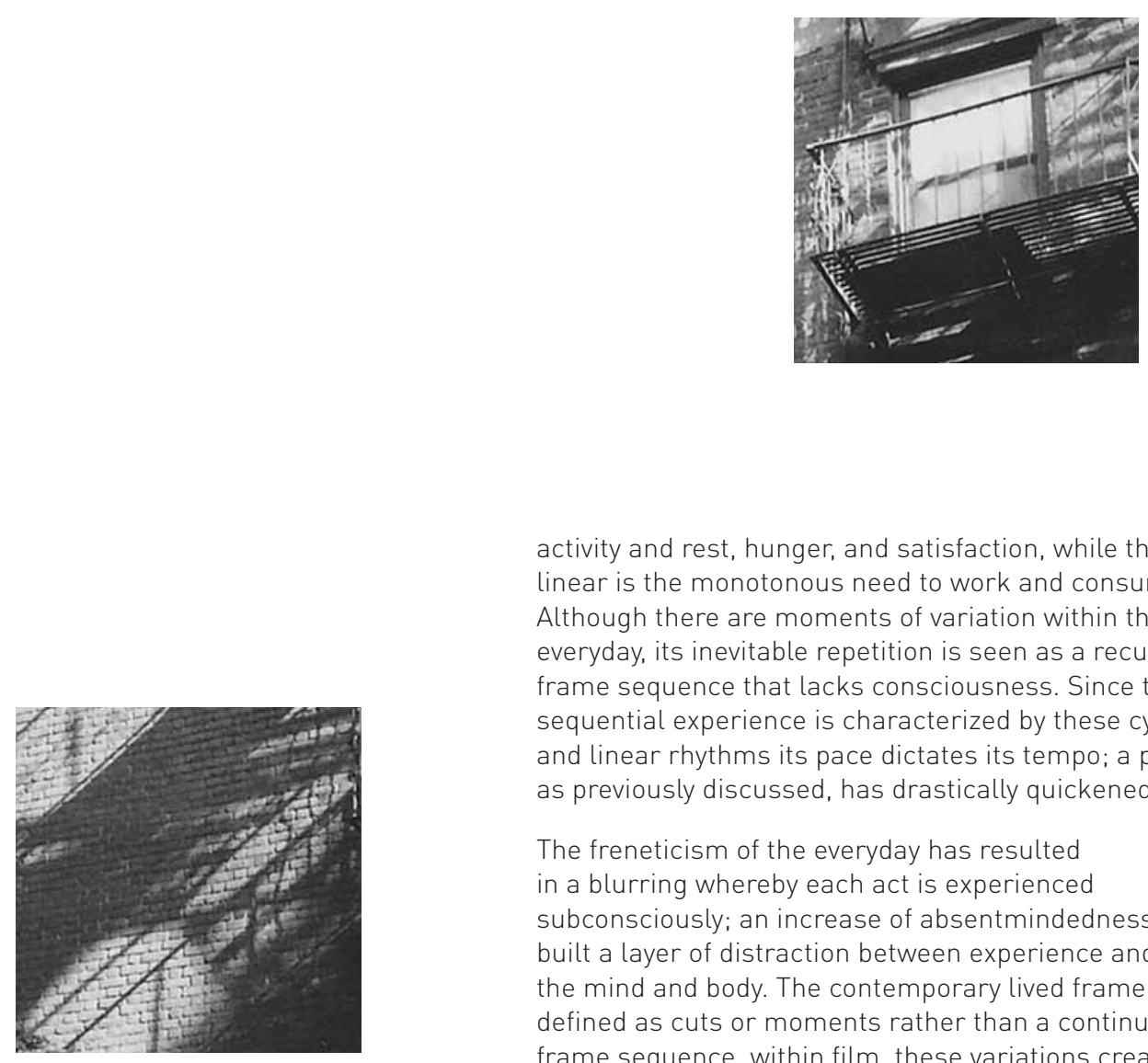

activity and rest, hunger, and satisfaction, while the linear is the monotonous need to work and consume. Although there are moments of variation within the everyday, its inevitable repetition is seen as a recurring frame sequence that lacks consciousness. Since the sequential experience is characterized by these cyclical and linear rhythms its pace dictates its tempo; a pace, as previously discussed, has drastically quickened. ${ }^{21}$

The freneticism of the everyday has resulted in a blurring whereby each act is experienced subconsciously; an increase of absentmindedness has built a layer of distraction between experience and the mind and body. The contemporary lived frame is defined as cuts or moments rather than a continuous frame sequence, within film, these variations create a quickening and slowing of time. The reading of architectural space, similar to filmic frames, depends on the density of and within a succession of frames or spaces. ${ }^{22}$ Therefore, by referencing speed cinema and slow cinema, spatial and perceptual collision take a montagist approach to create contrasting moments of quickening and slowing. For Eisenstein, rapid juxtaposition was used to condense the experience of space and time, whereas, Tarkovsky used the internal rhythm, of time and movement, both of and within frames to elongate space and time. In this sense, the contemporary everyday is experienced like an Eisenstein film, with varying viewpoints shown in quick cuts, and yet by decreasing the density of and within architectural frames, an increasing presence and 
habitability within space begins to slow the mind and body. To create an architecture of slowness, contrasting moments or thresholds of juxtaposition enable varying

Thresholds of Consciousness; levels of consciousness to map the narrativization of slowness.

Thresholds are considered to be a passage or pause that define an entrance or variance between two or more spaces, and yet, it is also seen as a moment of presencing. According to German philosopher, Martin Heidegger, "a boundary is not that at which something stops but, as the Greek recognized, the boundary is that from which something begins its presencing." ${ }^{23}$ For Heidegger, presencing signifies a state of awareness whereby apperception occurs, which is defined as the fully conscious perception or experience of a thing. In this sense, thresholds are not merely the linker and separator of spaces, rather, they delineate moments of consciousness within the everyday monotony. In architecture, the gaps - corridors, doors, staircases, and ramps - are typically perceived as spaces for speed since they contain the most movement. As previously discussed, Bernard Tschumi, considers the gaps to be conscious moments inserted between two or more events within an expanded sequence. ${ }^{24}$ For Tschumi, the gap, along with some other architectural elements, clearly reference the editing technique, be it cut, dissolve, prolonged, etc., used by an architect when choreographing the inhabitation. 

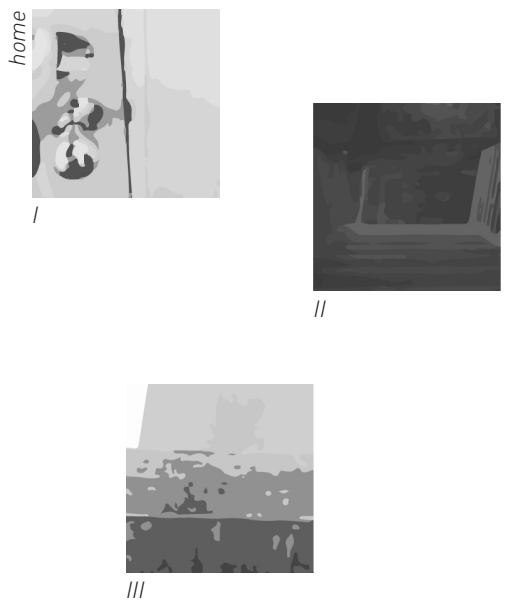

\section{Door}

Home, bedroom Home, bathroom Home, exit _ I

Car

Clarkson, garage

Clarkson, exit

Clarkson, platform

Train, entrance $I V$

Union, platform

Union, concourse 1

Union, concourse 2

Union to Subway 1

Union to Subway 2

Subway

Dundas, ex. 1

Dundas, ex. 2

$A M C$, exit

325 Church, ent. $1 \quad V$

325 Church, ent. 2

325 Church, ent. 3

325 Church, studio ent.
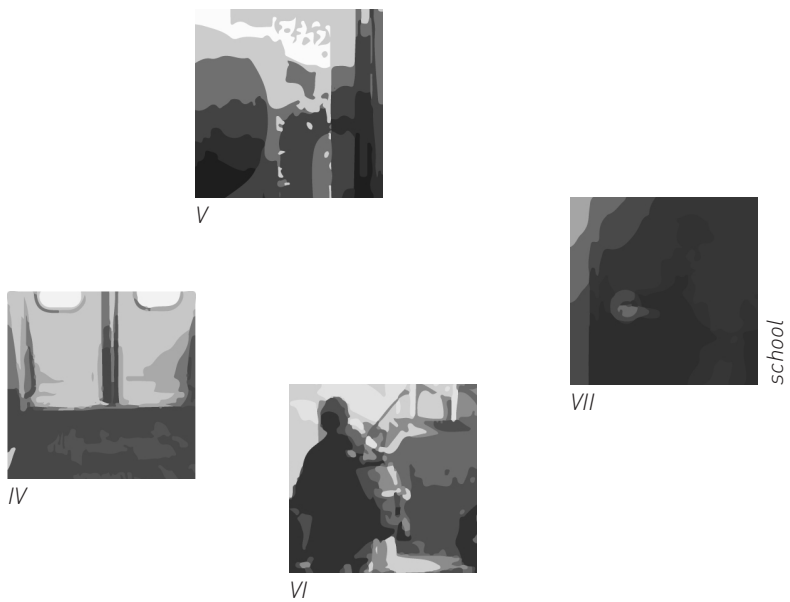

Staircase

Home, upstairs

Home, interior III

Home, exterior

Clarkson, ex.

Clarkson, basement

Clarkson, platform

Train, middle [2]

Union, platform

Union, concourse

Union to Subway, ent. 1

Union to Subway, ent. 2

Subway

AMC

325 Church, interior VI
Union, concourse

Union to Subway

Home, entrance

Clarkson, basement

Train, Clarkson to Union

Union to Subway

Subway, Union to Dundas

Subway to AMC

325 Church entrance

325 Church, studio ent. VII 


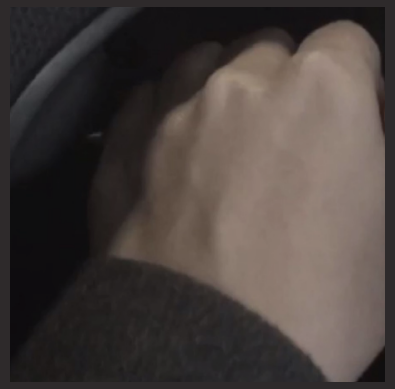

Thresholds of Consciousness

Lived Montage

The first film, Thresholds o Consciousness, is assembled in montage whereby cuts are used between frames to condense space and time
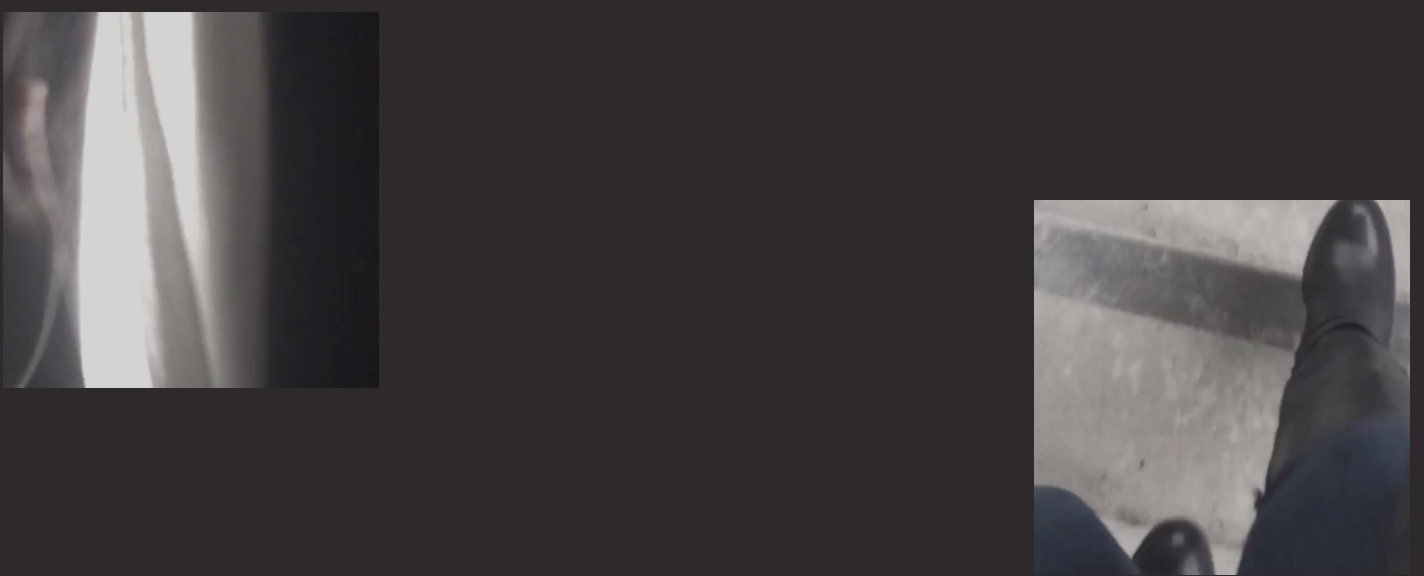

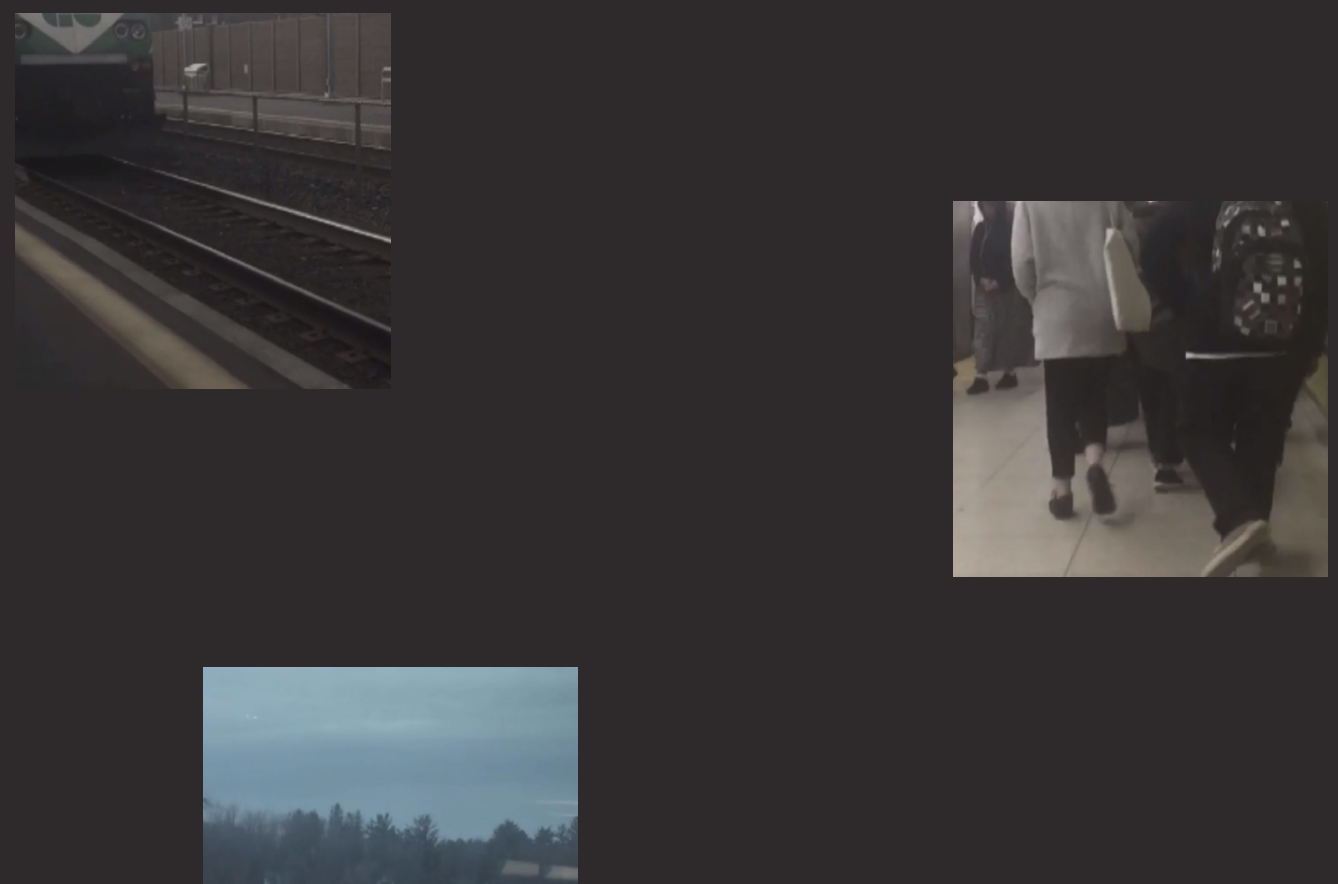

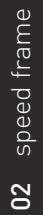

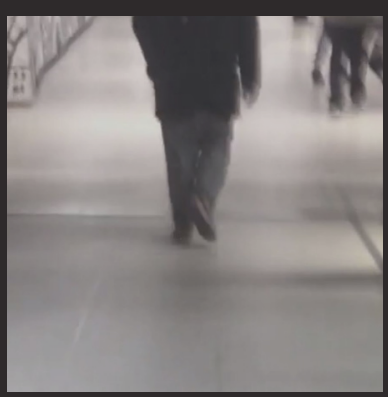


The everyday resides within a culture of speed whereby the relationship between physical and cognitive presence is limited by distractions. This absentmindedness is the result of the everyday monotony where unconscious habitual acts cannot form attachments with individuals, thus, ceasing to construct memories due to the lacking presence in everyday life. For French philosopher, Henri Bergson, the afterimage' is when the image of a previous object overlays upon a new one to create an expanded present. ${ }^{25}$ Considering Bergson's Circuit Diagram as a launching point for understanding temporal inhabitance, it is only with presence that individuals can begin to slow experiences.

The design experiment, Lived Montage, considers how, and if, speed affects the relationship between perceiver and perceived or subject and object. From Montage as Stills, it is deduced that frenetic speed is the main factor which alters the inhabitants felt presence within architecture. As the friend slowed down they began a dialogue between the mind, body, and space, in turn, creating attachments, known as place attachments, between subject and object. What became apparent with the previous design research was the disconnect between the individual and spaces for strictly movement, including corridors, doors, staircases, and ramps. A prominent example is seen with the second-floor corridor that leads to the offices. During the slowed walk the friend describes the stark white passage as, “quite mundane...it was as if they needed a hallway so they just made a hallway." From the description emerges an understanding of the varying levels of presence, cognitive and physical, within spaces for motion and stillness. In this sense, when spaces are solely designated for movement they tend to negate the emotive qualities associated with place.

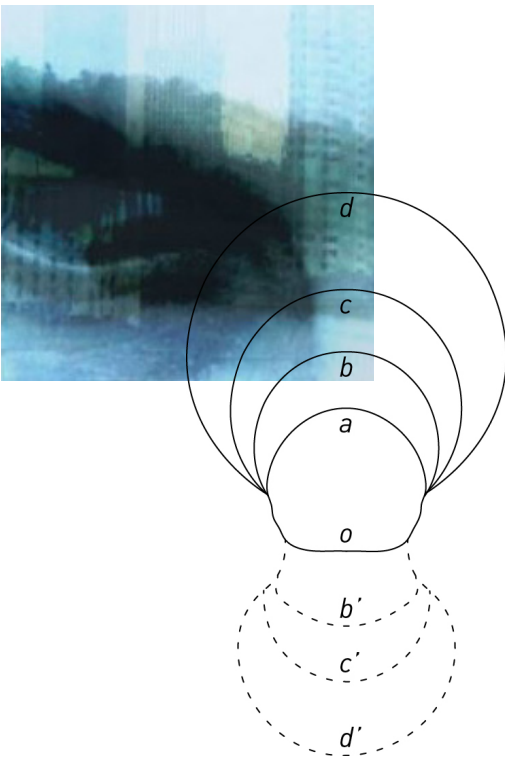

Circuit Diagram; Matter and Memory

1896

Henri Bergson

According to Bergson, the object image [o] is sent towards the mind as the memory image $[a]$ is simultaneously superimposed to create our perception. 

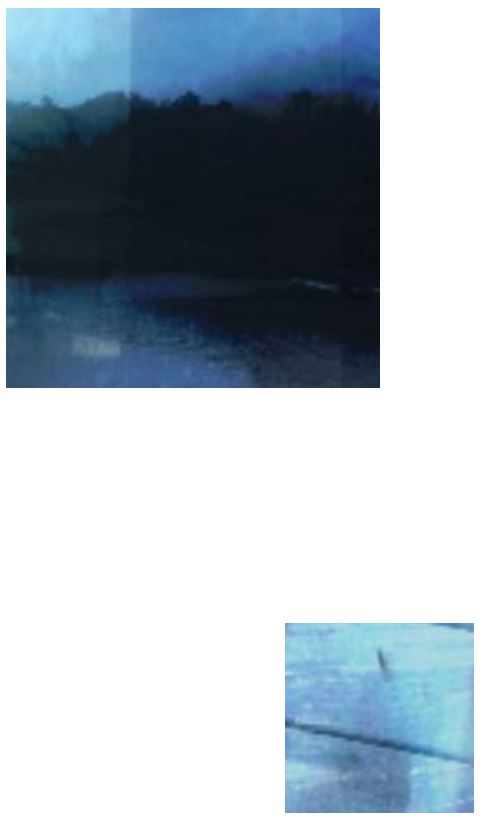

f26

Still

Memory Overlay; Lived Montage

While assembling the first iteration, Thresholds of Consciousness, of the experiment, Lived Montage, it became clear that it was portraying frenetic speed rather than slowness. A distinct sequence whereby the train ambassador announces the arrival to Port Credit station superimposed in my mind with a memory from Japan of my partner, who has resided in Port Credit all his life. Like La Jetée. the overlapping of one event with another revealed what Thresholds of Consciousness was lacking: attachment.
Within this design research, presencing was further analyzed to understand how the everyday monotony, rather than a new experience, can create moments of consciousness. The two films capture the varying gaps from my daily commute from home to school, this includes corridors, doors, staircases, and ramps. The first film, Thresholds of Consciousness, is assembled in a montage whereby cuts are used between frames to condense space and time. As a result, the quick cuts and varying perspectives creates a dizzying film representative of the monotony of the everyday. While assembling the first film it became clear that the moments of consciousness were limited to distinct transitional zones from $\mathrm{A}$, home, to $\mathrm{B}$, school; the mind was fixated on getting to these markers in order to complete the trip and arrive at school. The single presencing moment occurred when I was reminded of my partner who resides in Port Credit and currently works abroad as the train ambassador announced the arrival to Port Credit Station. Experiences of heightened emotion develop a dialogue between subject, or people, and object or place; as previously discussed this creates inherent attachments between the two. The understanding of the overlapping and viscous layers within the everyday formed the second film, Memory Overlay. The film was assembled by dissolving memories from Japan over the everyday commute. Although the second film was denser than the first, there were moments whereby the layers thinned in order for slowness to take over. From the second film, it was deduced that the contrast between density and emptiness heightens the transition between frenetic speed to slowness. 


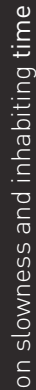
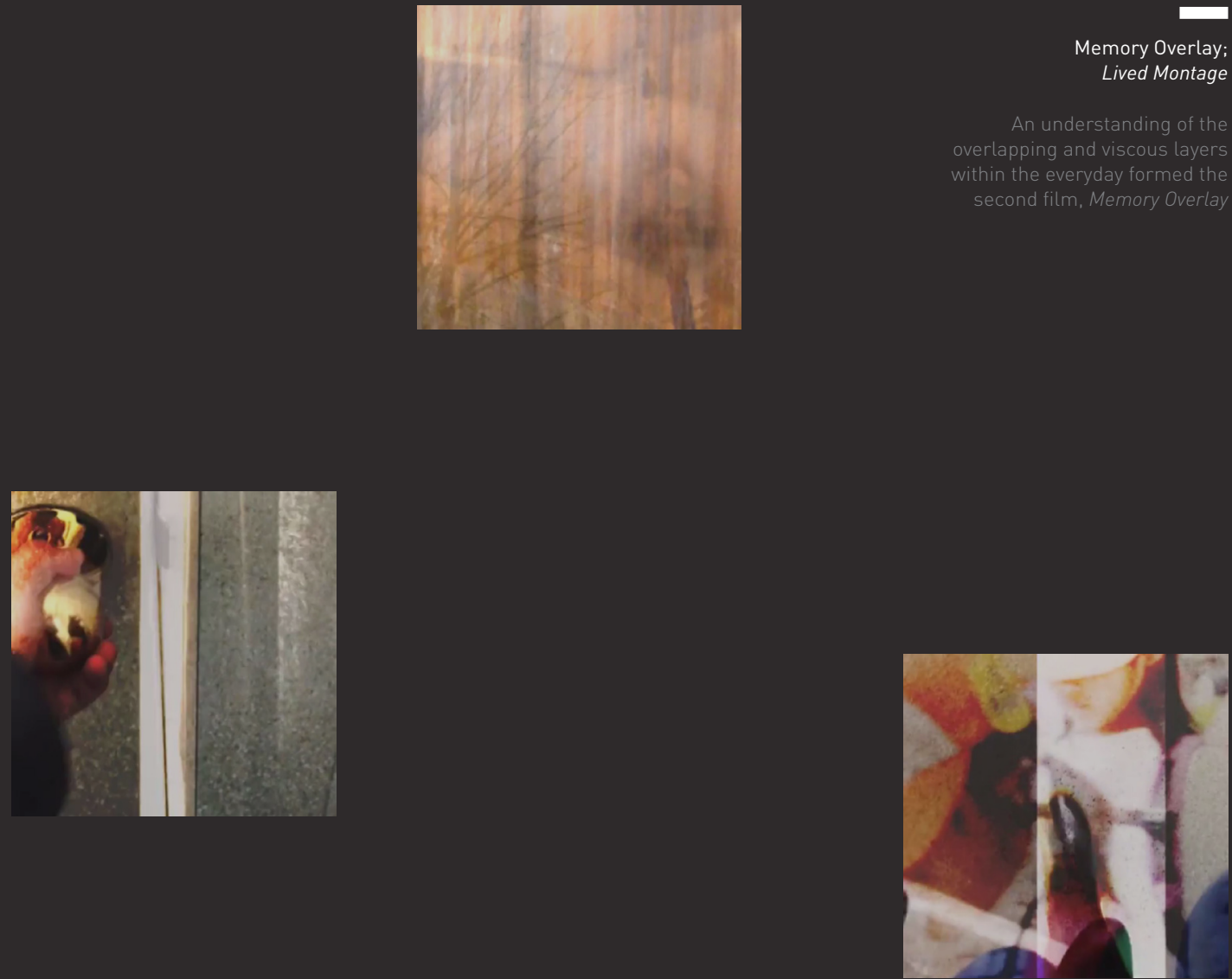

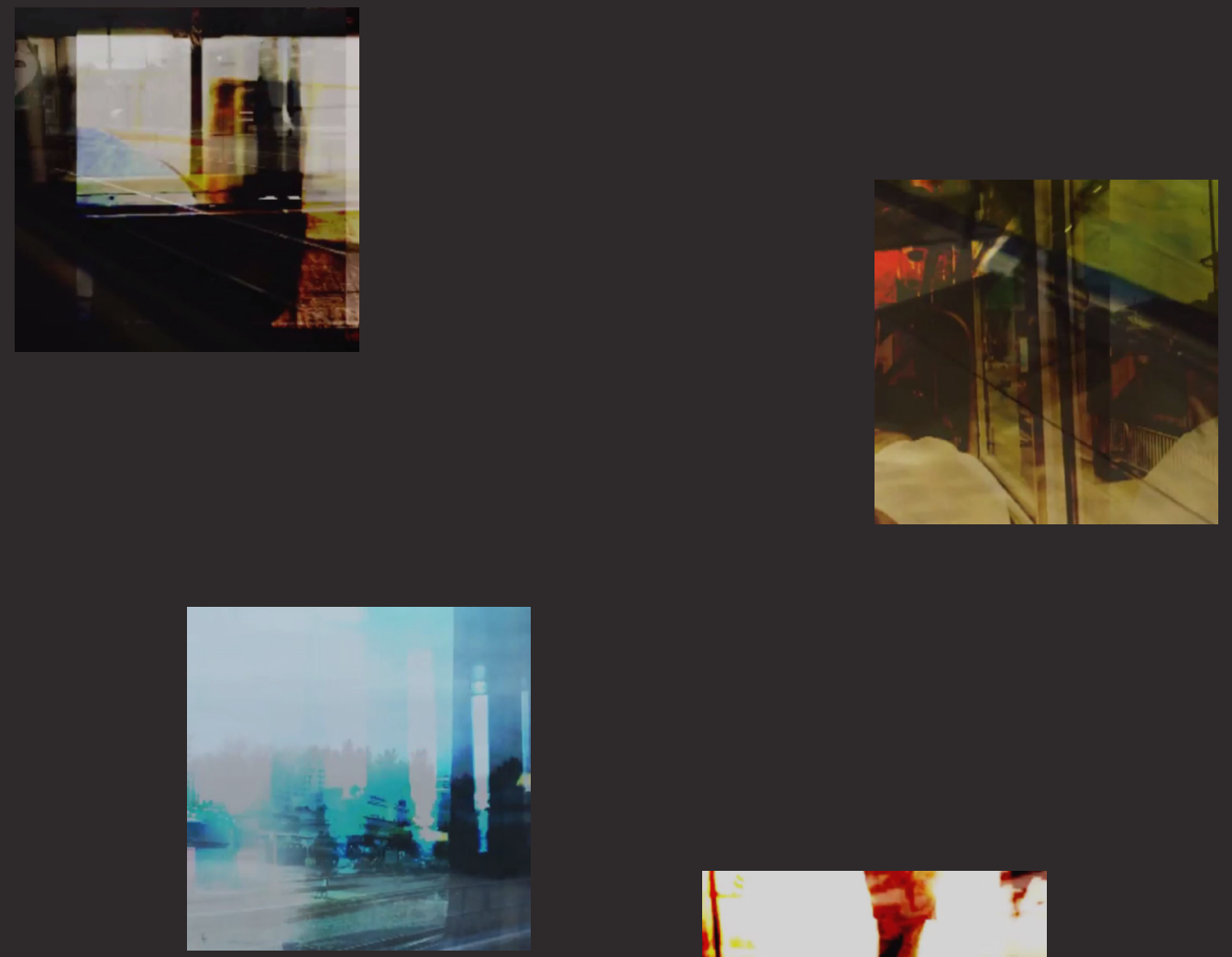

0
E
0
$\frac{0}{5}$
0
0
0
0
un
ฐ

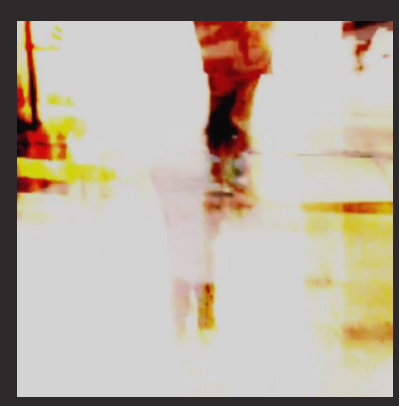




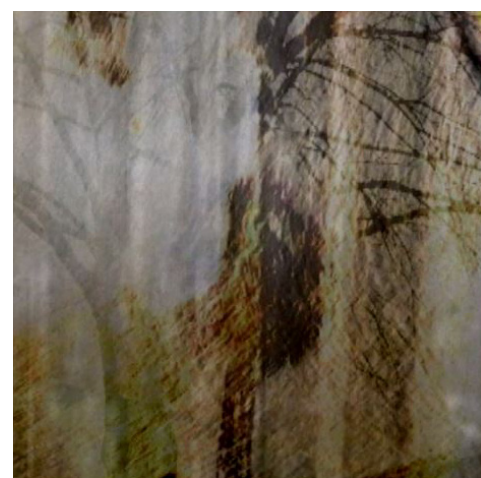

mississauga_osaka

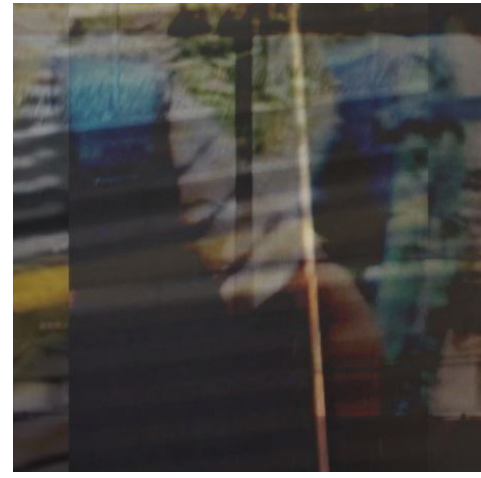

mississauga_kyoto

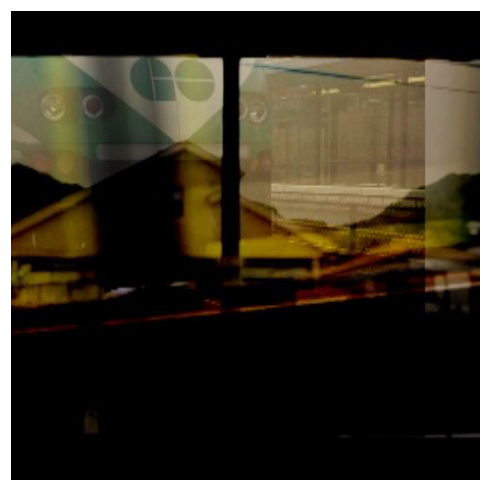

mississauga_osaka
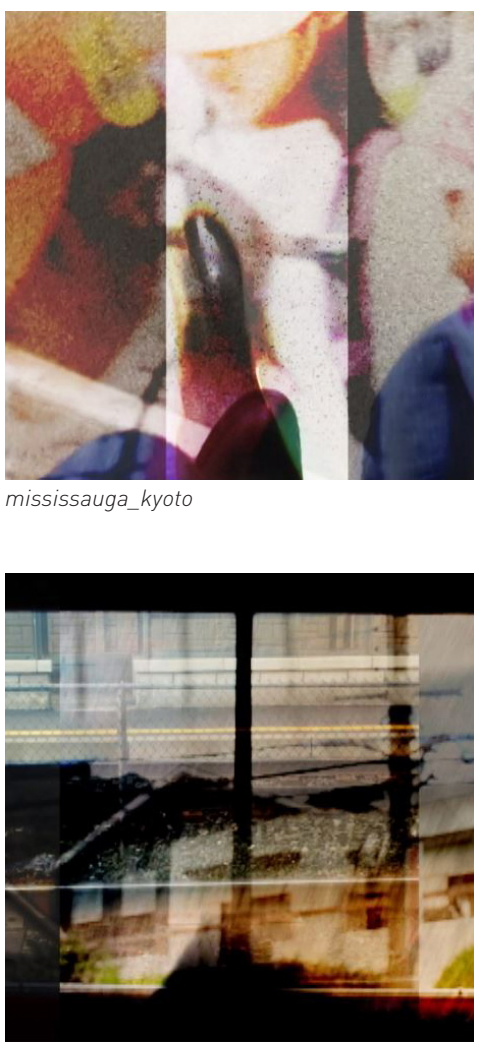

mississauga_osaka

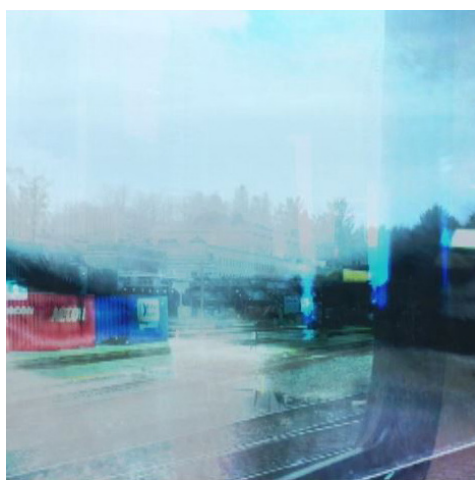

port credit_tokyo 


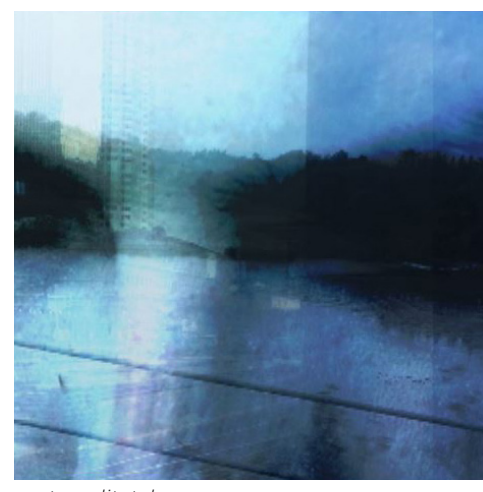

port credit_tokyo

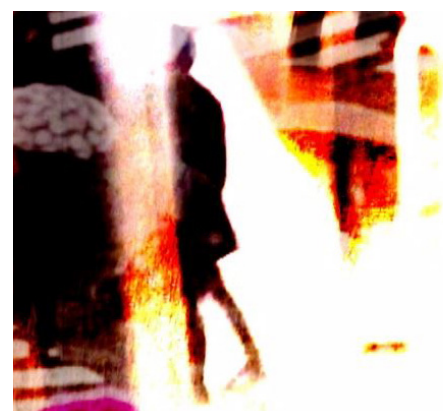

toronto tokyo

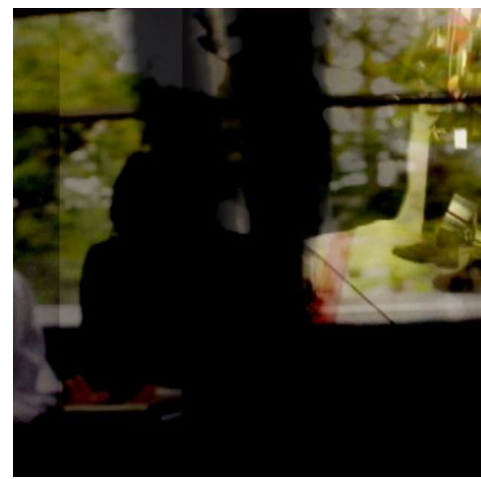

toronto_osaka

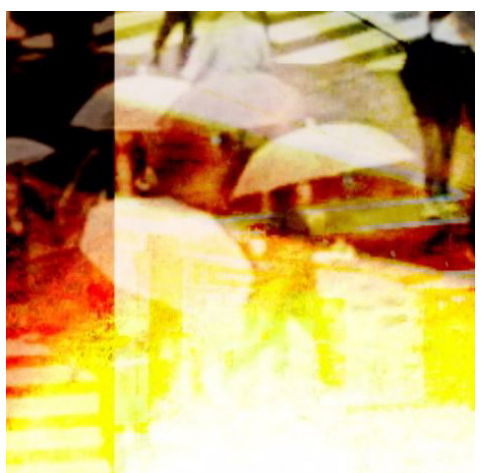

toronto_tokyo

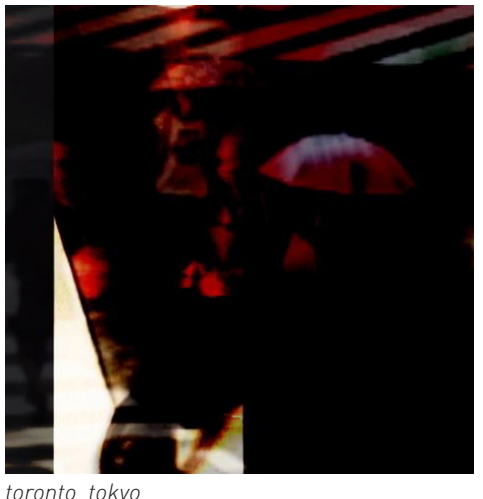

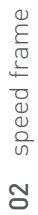

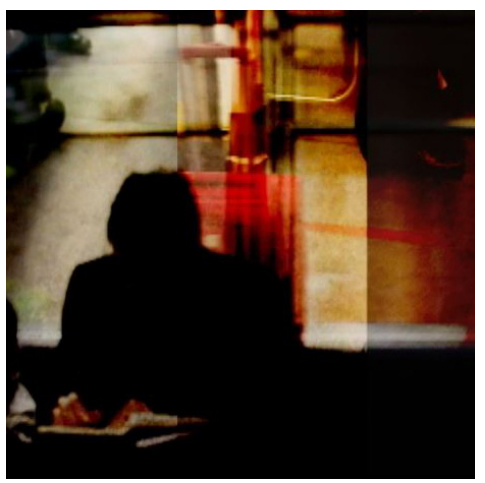

toronto_osaka 

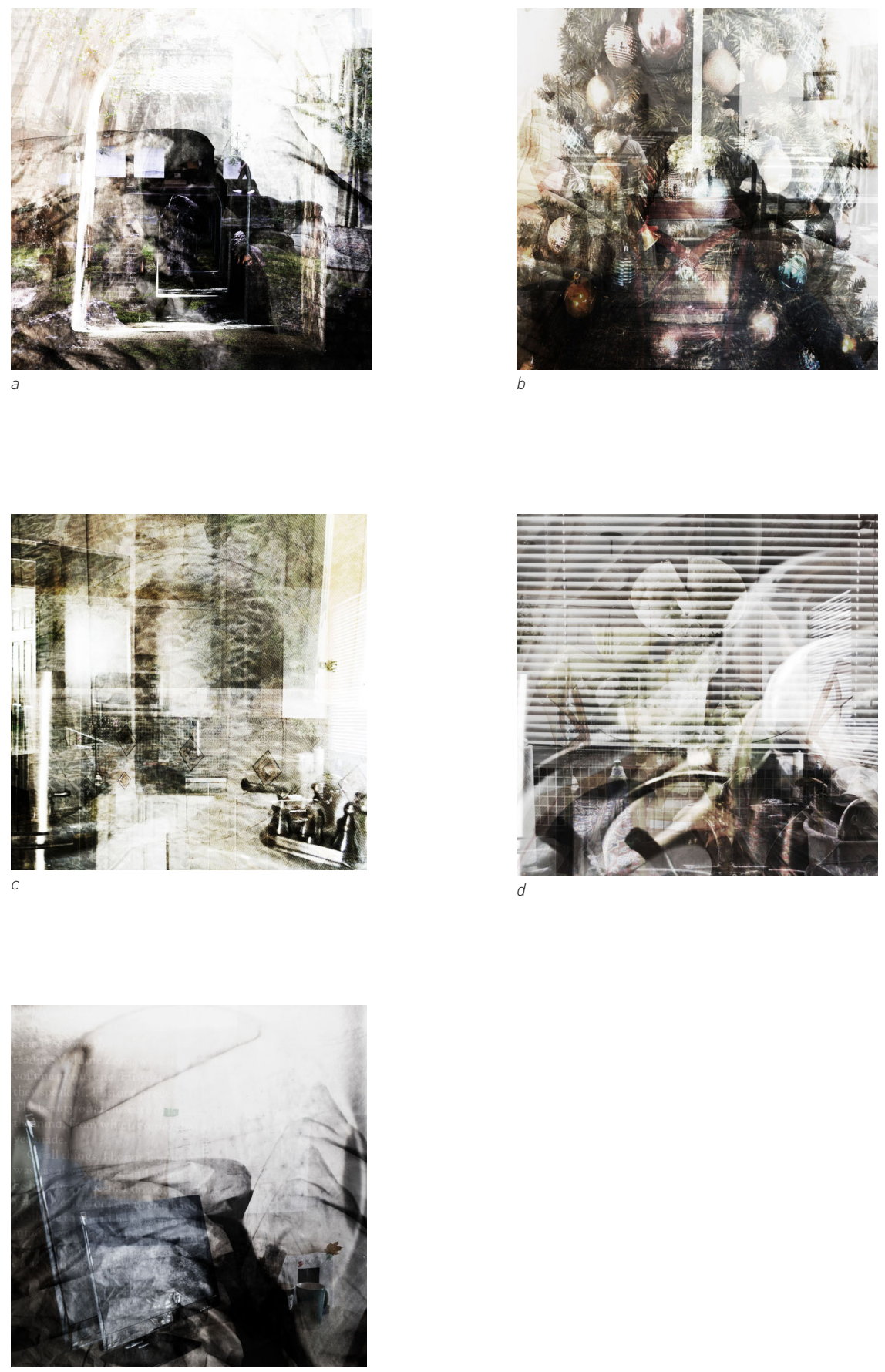
Everyday Overlay Study

\section{a_Resting \\ b_Gathering \\ c_Sustaining \\ d_Washing \\ e_Working}

Referencing Bergon's Circuit

Diagram, this study overlays an

object or present image from my everyday lincluding various

spaces in my home) with a memory image from my trip to Japan in 2016 to portray the superimposed experience of the everyday. The result collides the monotony of the everyday with fond and emotive memories in order to create a thick and viscous perception. 
Notes

1 Gilles Deleuze, Cinema 1: The Movement-Image, trans. Hugh Tomlinson and Barbara Habberjam (London: The Athlone Press, 1986), 1.

2 Bernard Tschumi, Architecture and Disjunction (Cambridge: The MIT Press, 1996), 167.

3 Andrei Tarkovsky, Sculpting in Time: Reflections on the Cinema, Trans. Kitty Hunter-Blai (Austin:University of Texas Press, 1987), 119.

4 Manfredo Tarfuri, The Sphere and the Labyrinth (Cambridge: The MIT Press, 1987), 56.

5 Ibid., 71.

6 Giuliana Bruno, Atlas of Emotion: Journeys in Art, Architecture, and Film (London: Verso, 2002), 57.

7 Ibid., 5

8 Tschumi, Architecture and Disjunction, 165.

9 Richard Koeck, Cine-scapes: Cinematic Spaces in Architecture and Cities (Abingdon: Routledge, 2013), 77.

10 Ibid

11 Tarkovsky, Sculpting in Time: Reflections on the Cinema, 117.

12 Pallasmaa, The Architecture of Image: Existential Space in Cinema (Helsinki: Rakennustieto Publishing, 2007), 18.

13 Walter Benjamin, The Work of Art in the Age of Its Technological Reproducibility, and Other Writings on Media (Cambridge: The Belknap Press of Harvard University Press, 2008), 35. 
14 Pallasmaa, The Architecture of Image: Existential Space in Cinema, 18.

15 Bruno, Atlas of Emotion: Journeys in Art, Architecture, and Film, 56.

16 Ibid., 58.

17 Benjamin, The Work of Art in the Age of Its Technological Reproducibility, and Other Writings on Media, 37.

18 Bruno, Atlas of Emotion: Journeys in Art, Architecture, and Film, 66.

19 Benjamin, The Work of Art in the Age of Its Technological Reproducibility, and Other Writings on Media, 40.

20 Henri Lefebvre "The Everyday and Everydayness" Yale French Studies, no 73 (1987): 10.

21 Robert Levine, A Geography of Time: The Temporal Misadventures of a Social Psychologist, or How Every Culture Keeps Time Just a Little Bit, (New York: Basic Books, 1997), 3.

22 Bruno, Atlas of Emotion: Journeys in Art, Architecture, and Film, 57

23 Martin Heidegger, "Building, Dwelling, Thinking," in Basic Writings (New York: Harper Perennial Modern Classics, 2008), 152.

24 Tschumi, Architecture and Disjunction, 165.

25 Charles Sherover, The Human Experience of Time: The Development of its Philosophic Meaning (New York University Press, 1975), 125.; Ibid., 371. 


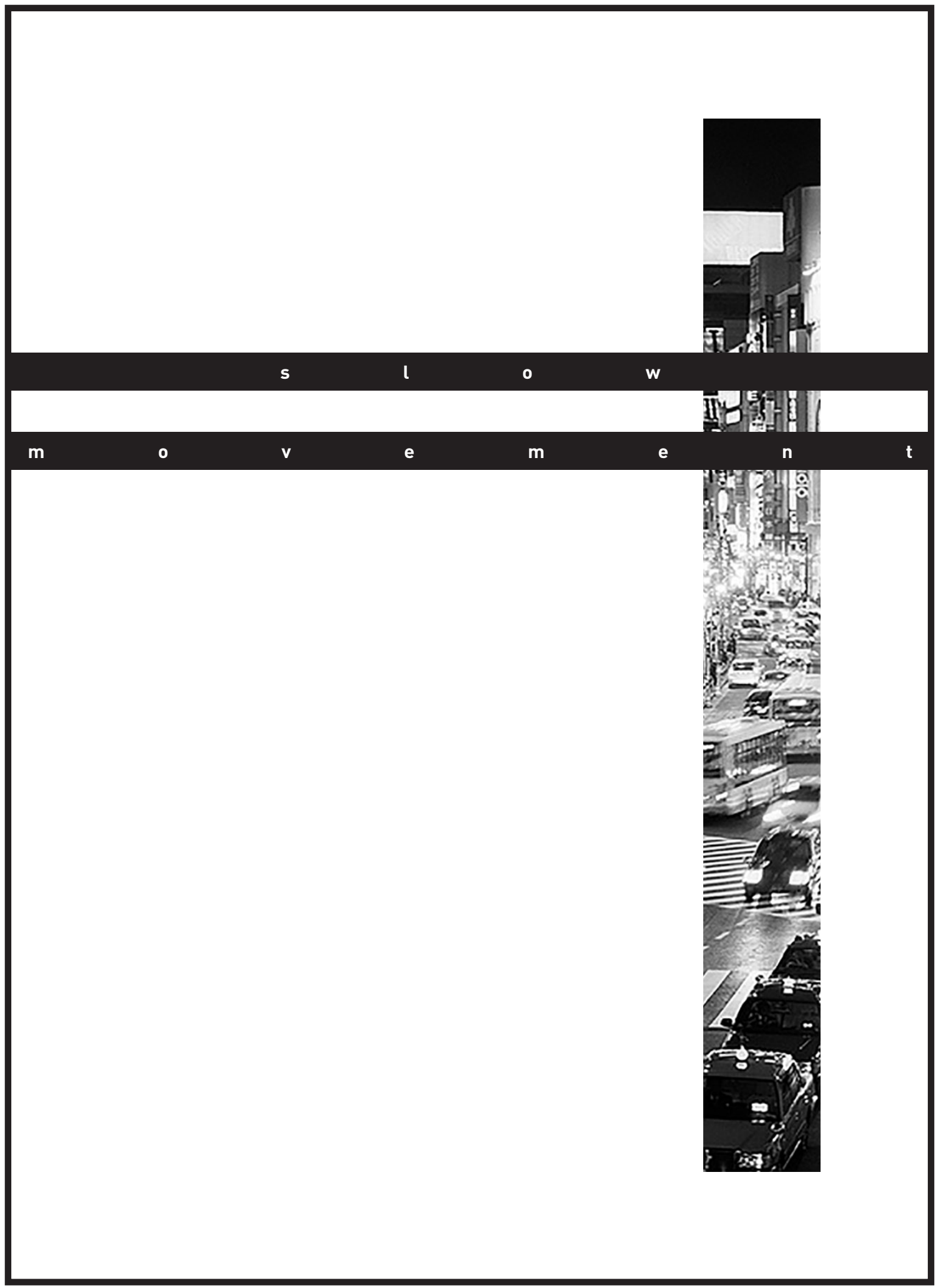


It is a cultural revolution against the notion that faster is always better. The Slow Philosophy is not about doing everything at a snail's pace. It's about seeking to do everything at the right speed. Savouring the hours and minutes rather than just counting them. *

This chapter explores the culture of slowness.

Shibuya Scramble 2010

Cain Doherty

\section{time poverty}

\section{1}

Within the last three centuries the contemporary world has seen an influx of energy towards efficiency; this results in a bond between time and speed, whereby, slow speed, or slowness, equates to wasting time. The idea of wasted time, stems from the culture of speed, where pace is prioritized within a goal oriented modern world.

In his Advice to a Young Tradesman, United States Founding Father, Benjamin Franklin, adjoins the idea of wasting time with wasting money in order to bring clarity to the importance of taking advantage of every millisecond, of every hour, of everyday, "He that can earn ten shillings a day by his labours and goes abroad, or sits idle one half of that day, though ought not to reckon that the only expense; he has really spent, or rather thrown away, five shillings besides." By considering idleness, or stillness, as wasted time, Franklin promotes solely monetary objectives in modern life; this distinct separation between stillness and motion divides time into two entities, leisure and work, therefore, the more work time gained the more leisure time can be wasted. ${ }^{2}$ Architectural professor and author, Günter Nitschke, furthers this phenomenon by referencing the Western concept of time as money, "Since one is compelled to 'waste' time moving from $A$ to $B$, one tries to shorten the lapses of 'empty' time by compressing experiential space through speed and ease of movement." ${ }^{3}$ This 

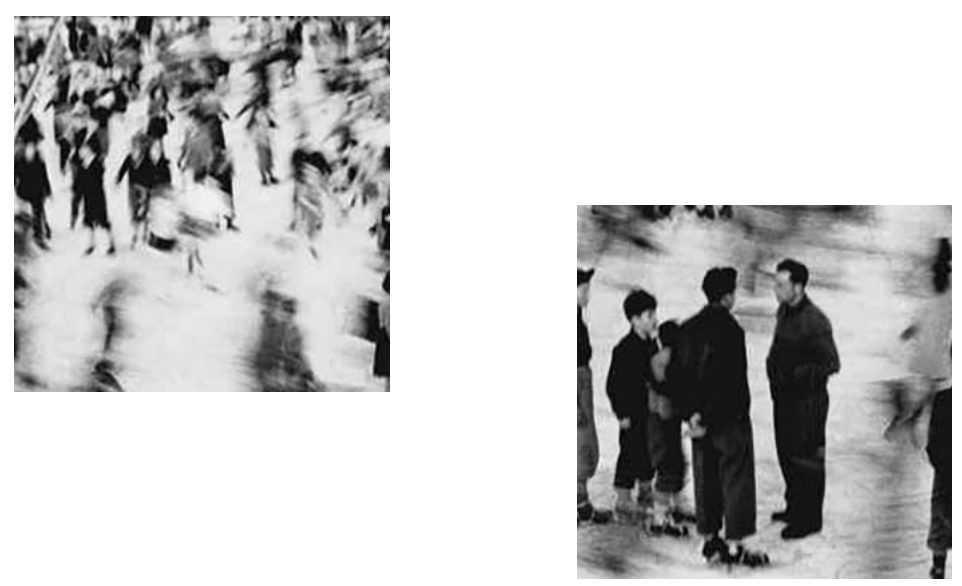

inadequate experience of everyday time is typically treated as though it can be acquired, saved, and spent; individuals are considered to be either time-rich or time-poor, and yet, monetary management cannot be used as reference for temporal inhabitance. ${ }^{4}$ As such, this exhaustive union has promoted an era of time poverty, whereby, each millisecond is considered within an everyday freneticism that's represented through fragmented moments of space and time. The inevitable consequence of coupling time and speed, with money, is the quickening of all aspects of everyday life, from people, objects, to information. ${ }^{5}$

This endemic situation is both innate, due to human mortality, and more important acculturated; although the contemporary world is confined by the omnipresence of speed, its impacts vary with regards to a specific culture's understanding of space and time. ${ }^{6}$ The essays, Time is Money and Space is Money, in Nitschke's From Shinto to Ando: Studies in Architectural Anthropology in Japan, discusses dissimilar perspectives towards the two dimensions within Western and Eastern culture; Western culture considers time as linear, striving for an abundance of leisure time through spatial compression, whereas, Eastern culture considers time as cyclical, striving for spatial expansion through temporal inhabitance. ${ }^{7}$ This variance identifies a hierarchical disconnect between the two dimensions in the West and the East. By comparing the final notes in each essay, the enmeshed relationship between 


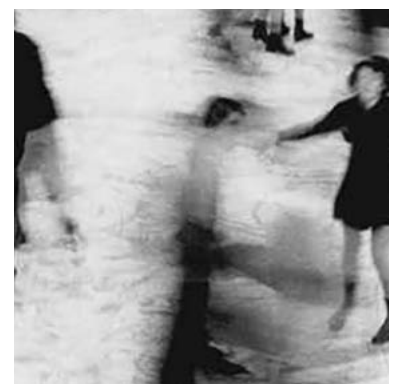

space and time illustrates the overpowering control of speed on life; the Time is Money quotation is referenced again, in its entirety, for convenience:

Time is Money

"America's 'places' are far from each other. Since one is compelled to 'waste' time moving from $A$ to $B$, one tries to shorten the lapses of 'empty' time by compressing experiential space through speed and ease of movement. Time is gained by 'killing' (compressing) space."

Time is Space

"Since Japanese 'places' tend to be very close to each other, the tendency is to expand space by increasing experiential time through the reduction of speed and the obstruction of movement. Space is created by 'killing' (slowing down) time." 9

Time poverty traces back to the culture of speed, thus, as Nitschke clarifies with the above quotation from Time is Space, in order to combat the contemporary fascination with speed, slowness or slowing down enables an expanded experience of both space and time. Thereby, through slowness, an individual is able to reestablish the lost connection between time and experience. This thesis uses space to manipulate temporal inhabitance through contrasted moments of presence, both mentally and physically, which foster spatial and perceptual connectivity. 


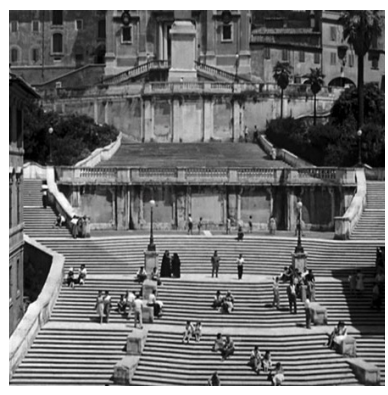

The Slow movement originated in 1986 with Italian culinary writer, Carlo Petrini, as the international Slow Food movement, a campaign against the opening of a fast food chain, McDonald's, besides the renowned Spanish Steps in Rome, Italy. ${ }^{10}$ Petrini launched the movement in order to advocate for a cultural shift from frenetic speed towards slowness with regards to the pace of life, specifically with fast food culture. Its initiating campaign turned sub-movement, Slow Food, represents a belief that food, as well as its industry, should be cultivated, prepared, and consumed at a relaxed or slowed pace. Their manifesto states: "A firm [defense] of quiet material pleasure is the only way to oppose the universal folly of Fast Life...Our [defense] should begin at the table with Slow Food;" 11 Thereby, harvesting fresh, local, and seasonal produce, made with generation old recipes for friends and family, the movement reintroduces pleasure into necessary habitual acts. ${ }^{12}$ In his seminal book, In Praise of Slowness, Canadian journalist, Carl Honoré, defines the Slow philosophy as not, "... doing everything at a snail's pace...[but seeking to] live at what musicians call the tempo giusto-the right speed;" in other words, by advocating for both moments of quickening and slowing, the movement promotes balance within everyday life. ${ }^{13}$ This balance allows individuals to regain control of their daily tempo by disentangling from all forms of the cult of speed.

From its conception, the Slow philosophy has permeated into various spheres of life, some of which

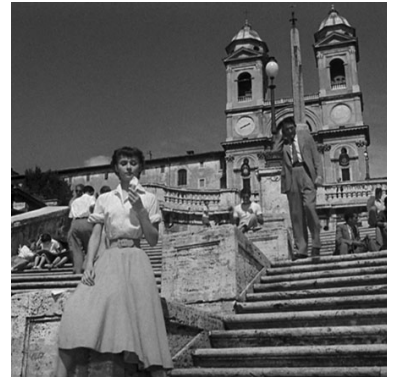




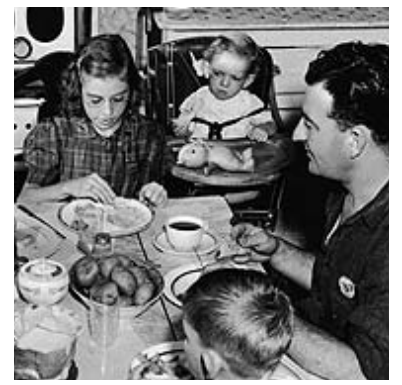

$\mathrm{f33}$

Christmas Lunch 1950

Unknown

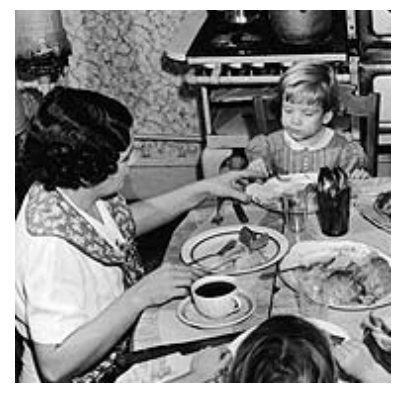

have popularized into their own sub-movement; various areas impacted by slowness include the city, cinema, fashion, goods, photography, and, as previously mentioned, food. This thesis highlights two of the spheres of life mentioned above, as their principles further various elemental ideas within an architecture of slowness, these include the city, and cinema.

Inspired by the Slow Food movement, the Citta Slow, or Slow Cities movement, was founded in Italy in order to resist homogenisation and globalization within cities; this sub-movement's manifesto contains fifty-five points geared towards slowing down and improving the quality of life, this includes cutting noise and traffic, promoting environmental technology, preserving local conditions, and increasing pedestrian friendly and green spaces. ${ }^{14}$ In this regard, "a Slow City is more than just a fast city slowed down. The movement is about creating an environment where people can resist the pressure to live by the clock and do everything faster," and yet, slowing down does not support technophobia or industrial disassociation, rather, it questions when and if specific technological changes improve the quality of life. ${ }^{15}$ Citta Slow, like Slow Food, is about reconnecting with oneself and the space one inhabits by heightening presence through fully conscious, or apperceptive, experiences within everyday life.

Prominent cinematic techniques associated with slow cinema, including the awareness of duration through temporal unfolding, originated before the Slow Food movement, specifically in the works of Andrei Tarkovsky and Aleksandr Sokurov. ${ }^{16}$ At the time of conception, the 


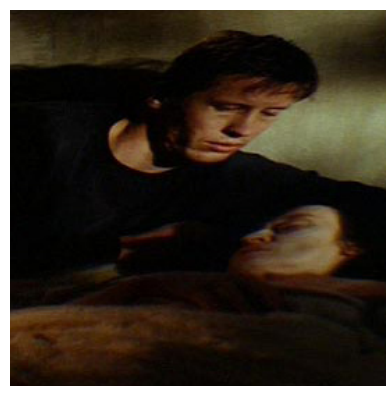

work was often referred to as a cinema of time; within the last decade, the term slow cinema has become popularized in the Western world for its emptiness, both in narrative and frame density, which allows individuals to descend into thought and contemplation while viewing a film. ${ }^{17}$ In this regard, "slow cinema can be seen as an unstructured film movement made up of disparate films and practices that are conceptualised as a grouping [due to a] comparable style." 18 The main component within this cinematic style is its ability to manifest time within the frame and the viewer by lengthening frame duration, as well, camera angles, distance, and movement is pertinent in expanding the perception of space and time..$^{19}$

Notes

1 Gleick, Faster: The Acceleration of Just About Everything, 239.

2 Nitschke, From Shinto to Ando: Studies in

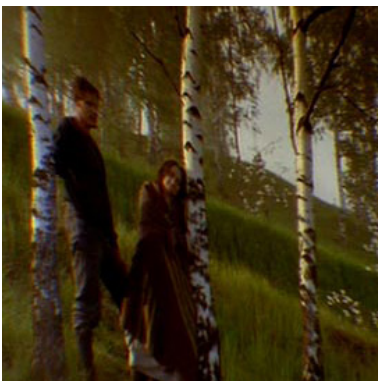
Architectural Anthropology in Japan, 34.

3 Ibid.

4 Carl Honoré, In Praise of Slownesss: Challenging the Cult of Speed (New York: Harper Collins, 2009), Adobe Acrobat eBook Reader, 29.; Gleick, Faster: The Acceleration of Just About Everything, 247.

5 Nitschke, From Shinto to Ando: Studies in Architectural Anthropology in Japan, 34. 


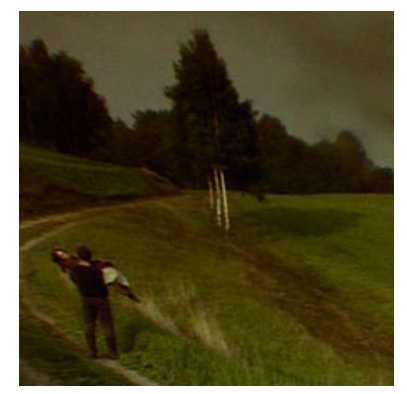

6 Honoré, In Praise of Slownesss: Challenging the Cult of Speed, 29.

7 Ibid.; Nitschke, From Shinto to Ando: Studies in Architectural Anthropology in Japan, 34-35.

8 Ibid.

9 Ibid., 35.

10 Honoré, In Praise of Slownesss: Challenging the Cult of Speed, 59.

11 Ibid., 15.

12 Ibid., 59.

13 Ibid., 15.

14 Ibid., 86.

15 Ibid., 87.

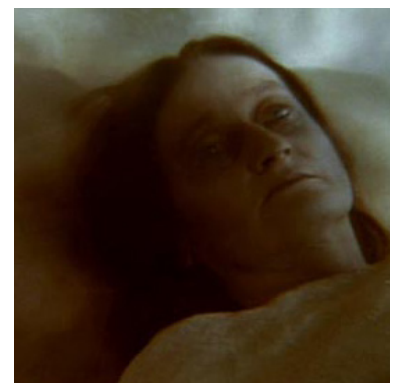

16 Tiago de Luca and Nuno Barradas Jorge, eds., Slow Cinema (Edinburgh: Edinburgh University Press, 2016), 4.

17 |bid., 2

18 Ibid., 4.

19 Ibid.,5; Ibid., 6. 


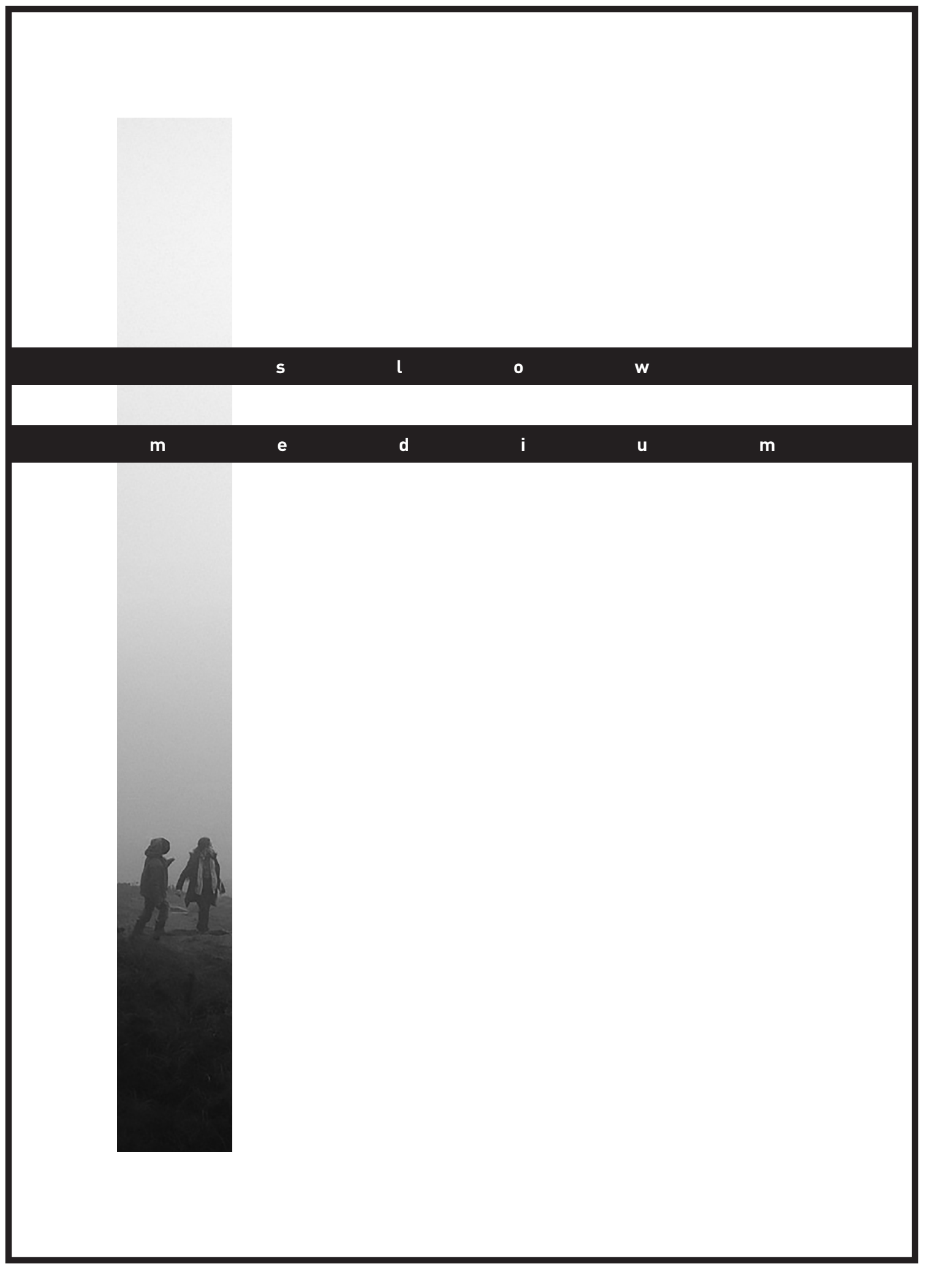


We behold, touch, listen and measure the world with our entire bodily existence and the experiential world is organized and articulated around the center of the body. Our home is the refuge of our body, memory and identity, which is in constant dialogue and interaction with the environment.

\section{duration}

This chapter explores the various elements that define slowness.

Walkers Emerge From The Fog 2014

Paudle Fearon

\section{1}

Duration is considered both the succession and overlay of distinct spatial and perceptual constructs. It collapses past, present, and future into a sequence of frames defined as the durée réelle or real duration. Within the everyday, real duration is experienced through a series of lived frames that actualize, moment by moment, as the mind and body progress through space and time; in this sense, "it is we who are passing when we say time passes." 1 For Bergson, flowing and indivisible time is defined as durational. It is experienced as the unwinding thread of moments, overlapping to create the continual unfolding of time, without which, "there would be only space, and space that, no longer subtending a duration, would no longer represent time. ${ }^{2}$ In this regard, temporal experiences are directly associated with spatial and perceptual collision, whereby, processions of varying speed and density influence the flow of time. As previously discussed, cinematography uses a similar methodology with film assembly, editing techniques are used to vary the sequence of and within frames in order to compress or expand space and time. Within architecture, alternating frame sequences begin to choreograph a performance with the mind and body, which leads an individual towards a mindful physical presence.

Presence is the apperceptive experience of a specific place or moment; it develops a dialogue between the mind, body, and the space it inhabits. With duration, the continuous, flowing, and ephemeral qualities of life transmit themselves into a thickened experience 

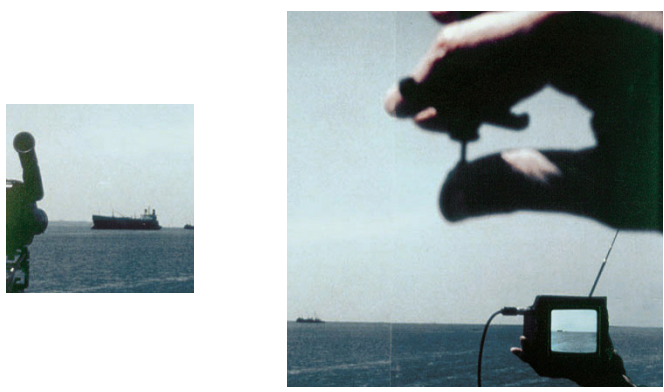

of space and time. The merging of these two entities establishes an architecture of embodiment through "the temporalization of space and the spatialization of time," which begin to see the two as ubiquitous. ${ }^{3}$ Nitschke, describes the merger of space and time as, "...two sides of a coin, indivisibly coupled. If one is preconditioned by nature or modified by man, the other Diller and Scofidio is affected. " With the acceleration of the everyday, time has begun to equate to speed and, in turn, is associated by the seconds it takes to get from location, A, to destination, B; its continuity, experience, and relationship with space is disregarded for efficiency. In Japanese culture, the awareness of time is prevalent in the Japanese sensitivity to the passing of hours, days, and seasons. This sensitivity is unlike the one associated with speed, rather, it stems from the idea that space and time were never fully separated. Contemporary Japanese architect Arata Isozaki defines this relationship:

Space could not be perceived independently of the element of time [and] time was not abstracted as a regulated, homogenous flow, but rather was believed to exist only in relation to movements or space...Thus, space was perceived as identical with the events or phenomena occurring in it; that is, space was recognized only in its relation to timeflow. $^{5}$

The Japanese believe that between several spatial and temporal events or things exists openings, holes, spaces or times between. ${ }^{6}$ This is defined as ma, a term 


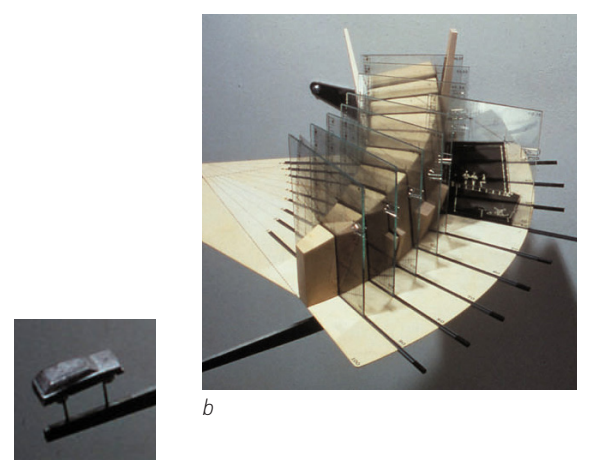

which can be described as an interval or boundary, similar to a gap, pause, or rest; it is the emptiness in haiku poetry, the negative or blank space in Japanese paintings, architecture, and gardens. Although ma is considered as the interval or threshold between space and time, it is ultimately seen as a link between two or more spatio-temporal moments within a procession; this reiterates Heidegger's belief that "a boundary is not that at which something stops but... the boundary is that from which something begins its presencing." 7 In this regard, ma is not merely the interval which links spatial and temporal constructs, rather, it demarcates moments of consciousness within a procession. Through the collapse of space and time as distinctly separate things, the everyday is no longer experienced solely spatially or temporally, but within a spatio-temporal lived frame whereby collision begins to presence the mind and body.

With architecture, spatial and perceptual succession is considered durational when collision or overlap of distinct architectural elements creates a gap or pause within a procession. When describing this collision of events mathematician and philosopher, Alfred North Whitehead, considers "every duration [to be apart] of other durations; and every duration has other durations which are parts of it." ${ }^{8}$ Considering a single moment to have no temporal or spatial extension; Whitehead disregards the conventional understanding of time as a series of moments for a more continuous, flowing and overlapping definition of time. Arguably, time is both a series of moments and continuous, since the human mind and body resides in continuous time 


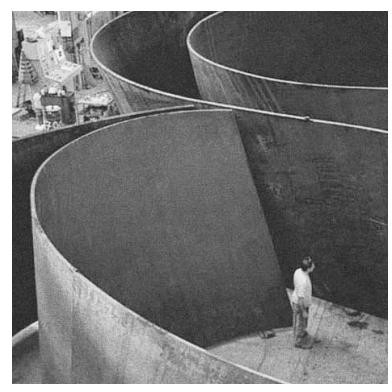

but experiences moments by flowing in and out of consciousness. In order to create an architecture of slowness, contrasting moments or thresholds of juxtaposition collide with one another to within varying levels of consciousness to map the narrativization of slowness.

Presence, as discovered with Montage as Stills, is the physical or cognitive state of being present in a specific place or moment. A heightened level of consciousness is the main component which begins to presence the mind and body. From Montage as Stills it was revealed that physical presence is attributed to movement, such that moving up a set of stairs exerts an exhaustive presence which brings awareness to the body; while cognitive presence or mental presence is attributed to perception, such that the recognition of a light streaming into an otherwise dark corridor begins to presence the mind and body.

Through presence, sustained attention allows one to become conscious of the dialogue between the body and the space it inhabits. Literary theorist, Hans Ulrich Gumbrecht, argues that events and experiences have palpable effects on the human emotions, senses, and bodies; creating a closely intertwined dialogue between the body and its experiences. In his retelling of an event, the viewing of a Japanese No and Kabuki drama, he emphasizes this linked relationship which "... ever so slowly begin[s] to let things emerge, [allowing one to] ...become a part of them." "Through apperception, this whole-body experience creates a mindful physical

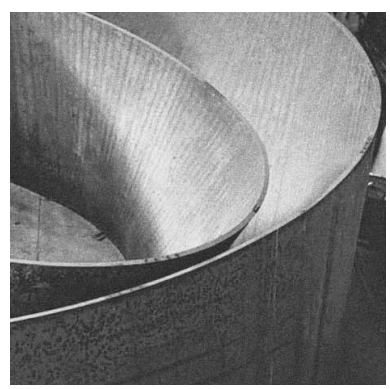




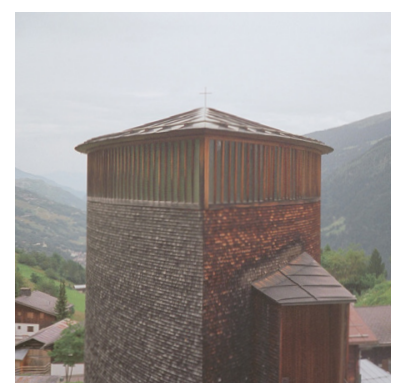

f38

Saint Benedict's Chapel 1988

Peter Zumthor

The journey to Saint Benedict's Chapel includes a narrow road that is driven, and a hill that is walked. This procession is the most important aspect of the chapel, as it slows the experience while heightening memory; the crisp air, lush grass filled with flowers, and the beautiful mountain views only intensify this experience. Within the chapel, the clerestory window allows an abundance of light to filter into the space, this juxtapositon brings awareness to the experiences while creating a moment of pause for the mind and body.

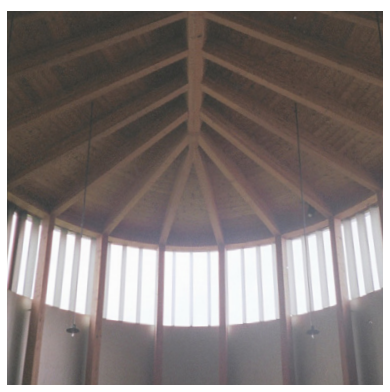

presence within the mind and body; mindfulness is the state of being present in a specific place or moment, one which is unaffected by the distractions of the everyday.

Mindfulness is considered the consciousness of one's internal and external experiences. Consciousness is the state of being awake which encompasses both awareness and attention. Awareness continually observes our inner and outer environments, whereas attention focuses our conscious awareness on a specific person, place or thing..$^{10}$ Awareness and attention intertwine to create the mindful body.

Nyanaponika Thera considers mindfulness as "the clear and single-minded awareness of what actually happens to and in us at the successive moments of perception." "11 It enables a deeper state of mental cognition whereby individuals observe and respond to their surroundings with heightened alertness and sensitivity. ${ }^{12}$ This sense of awareness slows down thought, speech, and action in order to bring cognition to the forefront by enabling individuals to pause and stop either mentally or physically. ${ }^{13}$ Human sensitivity activates a mindful physical presence which "hinges on the sense one has of the space where one is." 14 This sensitivity is found within the atmospheric qualities of architecture. Atmosphere establishes a mental and physical experience of mindfulness; a sensation which presences the mind and body.

Our everyday experiences are based on how we perceive our surroundings; and yet, the contemporary perception is a fragment of moments. Cognitive scientists consider our conscious mind to take time to extract patterns within the fragmented diversity of 


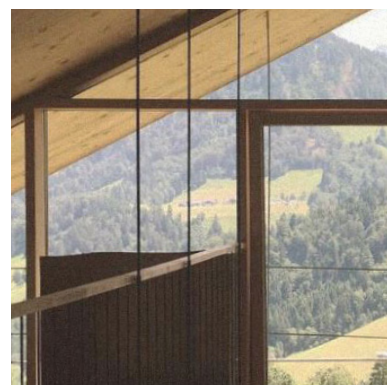

our experiences. ${ }^{15}$ This fragmentation is the result of the human ability to impede redundant stimuli from entering its consciousness. ${ }^{16}$ According to American psychologists Kirk Warren Brown and Richard M. Ryan, the everyday is experienced in a perpetual state of mindlessness, a "less "awake" [state] of habitual or automatic functioning [that may] be chronic for many individuals." 17 The value of mindfulness is evident in its ability to capture the clarity and vividness of consciousness in everyday activities; in turn, heightening the experience of the everyday. ${ }^{18}$

Although the experience of mindfulness manifests differently in each person, its overall function is to bring one into the present by sustaining attention on the mind and body. The experience, unbound from the restrictions of physicality, achieves an amorphous qualitative space within the mind. ${ }^{19}$ Within architecture, a dialogue emerges between the atmospheric qualities of space and its inherent ability to activate the senses. This intertwined relationship between mindfulness and atmosphere is pivotal in the creation of the mindful body. The mindful body is a state that one senses both mentally and physically. The term was introduced by German philosopher and author Gernot Böhme when describing atmosphere and its implications on mood. ${ }^{20}$ Mindful physical presence hinges on the interplay between the body and the mindful body. ${ }^{21}$ It brings cognition to the forefront in order to thicken experiences by presencing an individual's mind and body; in turn, creating the present body, one that begins to inhabit time.
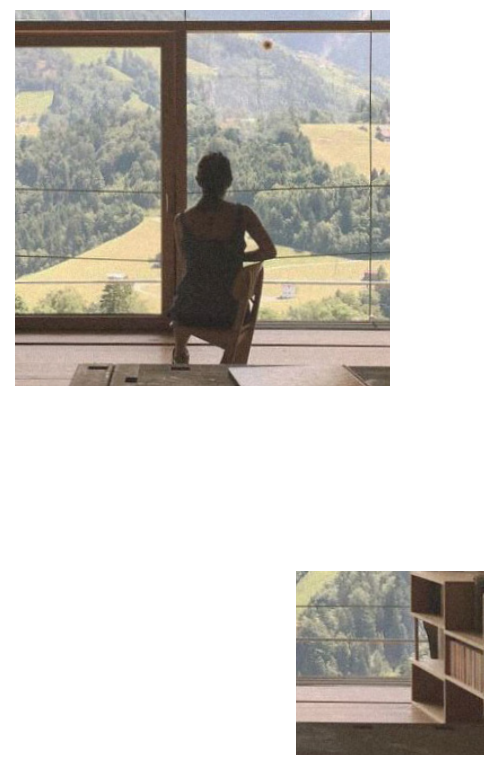

Blons Community Centre

2005

Bruno Klomfar 


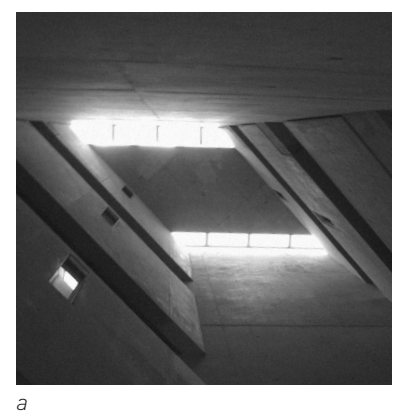

$\mathrm{f} 40$

Jewish Museum Berlin

1999

Peter Zumthor

a_Light

b_Darkness

The Jewish Museum Berlin by Daniel Libeskind uses movement, space, and memory to heighten the experience of time. Movement is pivotal to the atmosphere, from multiple stairs to ramped walkways, the body exerts an abundance of energy during the experience of the museum: in turn, heightening a physical presence. Procession is important to the museum, as all pathways connect back to one central point, thus emphasizing time as mnemonic.

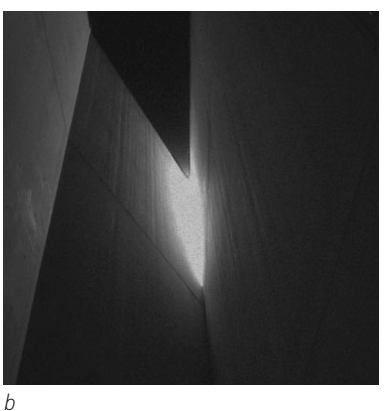

\section{place $\mathbf{4 . 3}$}

The difference between space and place is found in their natural association. Place is considered the character or spirit of a space, which reveals itself through time; in this sense, spaces are designed to create places. Nitschke describes place as "the product of lived space and lived time, a reflection of our states of mind and heart." 22 In our accelerated world, people spend more time mindlessly inhabiting spaces than they do creating profound attachments, thereby, making the engagement with spaces momentary and fleeting. As revealed with Montage as Stills slowing down, both physically and mentally, enables a deeper state of awareness that increases the ability to develop attachments to a particular place or moment. This heightened awareness and sensitivity is prevalent in the atmospheric qualities of architecture.

Atmosphere is the pervading feeling emitted by a specific place or moment that activates a mindful physical presence. ${ }^{23}$ It is the sense of being in the world; this idea of being implies a heightened level of consciousness through which the world is experienced. German philosopher, Hermann Schmitz, believes corporeal feeling to be the underlying sense affected by the atmosphere of a place or moment. ${ }^{24}$ Corporeality is understood as the physical experience of the material body, or one's bodily existence. For Gernot Böhme, "atmospheres are essentially spatial; more precisely, they are spaces pregnant with a mood." ${ }^{25}$ These moments are experienced by corporeal feeling, the mood penetrates the emotive state of an individual resulting in a heightened sensitivity that allows them to feel both themselves and the space by placing them in a specific spatial mood. 


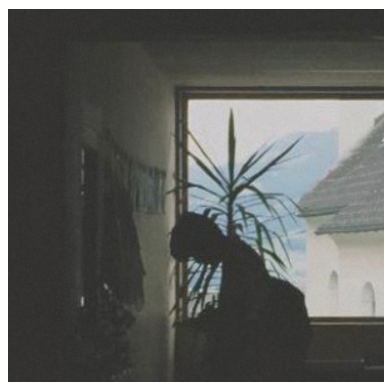

The emotive qualities of a certain place or moment have the ability to settle within the mind and body. As such, the individual becomes a part of the space

Volksschule Doren Doren Primary School 2003 and the space becomes a part of the individual. This Curkowicz Nachbaur profound dialogue between people and a place or moment defines being in the world; a dialogue which is contingent on the atmosphere of space.

When describing atmospheres, it is their intangible and sensuous elements, identified as atmospheric generators, which contain specific characteristics that produce memorable experiences. ${ }^{26}$ Within architecture, atmosphere establishes a sensation, different for each individual, which presences the mind and body. Juhani Pallasmaa considers these generators as, "qualities of matter, space and scale [which are] measured by the eye, ear, nose, skin, tongue, skeleton and muscle." 27 The experience of space is relative to the relationship amongst these spatial components. The elements of atmosphere float, unbound from the isotropic definition of space, interacting with the mind and body to create spaces of corporeality or corporeal presence. For Böhme and Schmitz, the immateriality of atmosphere is the nature of experienced presence. Schmitz illustrates this as, "...surfaceless as that of sound or silence, of the weather or a driving headwind..., of the water plunged into and crossed by a swimmer, as that of freely unfolding gesture, of feeling something in your own body." 28 To feel something but also feel yourself feeling is the essence of atmosphere.29

Experiences of heightened emotion develop a dialogue between subject, or people, and object, or place; this creates inherent attachments, referred to as place 


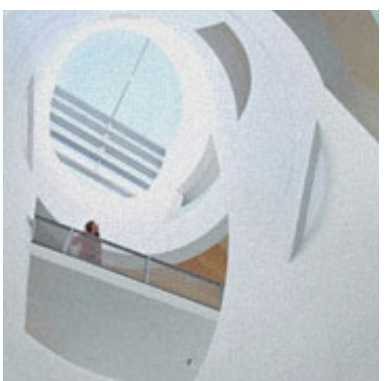

$\mathrm{f} 42$

The Glasgow School of Arts 1999

Steven Holl

The Glasgow School of Arts by Steven Holl questions the idea of shared time by wrapping three skylights with the circulation system of the entire building, thus, allowing for each user's time to overlap with another's. This interesting overlapping of time emphasizes the experience of time in a new way lindividual and shared).

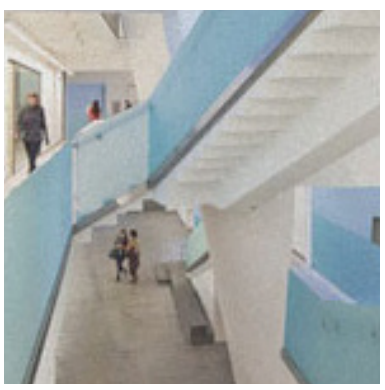

attachment. American Psychologists, Setha M. Low and Irwin Altman, define the concept as an intense affective bond between a person and a place or moment, which arises from emotion and feeling. ${ }^{30}$ From attachments or connections to specific places or moments, memories are formed in the shape of mental places. Portuguese architect, Paulo Providência, believes that, “...just like physical data, a place is mental or memorial data (memoria loci). The relationship between physical place and memorial place thus generates mutual interaction." ${ }^{31}$ Memories establish themselves within the mind in emotive and sensorial experiences of the body. Like mindfulness, memories are unbound from the restrictions of physicality, they embed themselves in the mind in varying ways. When recalled, they reappear in a collage of fragments, from the smell of freshly cut grass on a hot summer day, the sound of rain hitting our windows in the morning, to the feeling of jumping into the lake. ${ }^{32}$ The perceived experience is typically blurred in our memories, and yet, the body still remembers. American philosopher and professor, Edward S. Casey, believes there to be no memory without body memory, or the sensitive account to remembering. ${ }^{33}$

Our everyday experiences are defined as the blending or coming together of perception, the present, and memory, the past. ${ }^{34}$ For Henri Bergson, "memory, inseparable in practice from perception, imports the past into the present." ${ }^{5}$ Thus, the everyday becomes an amalgamation of what is experienced, remembered, and imagined; an expanded present, which overlays attachments on attachments. ${ }^{36}$ This understanding of an expanded experience of time is further defined in Bergson's Circuit Diagram where, as previously 


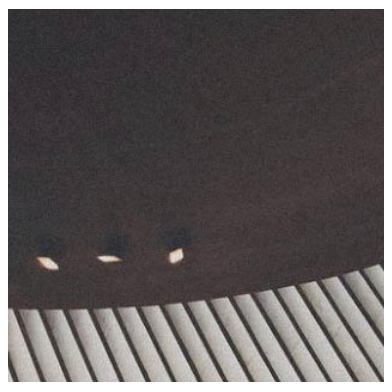

discussed, he illustrates the idea of the 'after-image. ${ }^{37}$ To Bergson, the 'after-image' is when the image of a previous object is overlaid upon a new one; in Rock Church turn creating what, philosopher and psychologist, William James defines, a 'specious present,' or an expanded present. ${ }^{38}$ The everyday is a flowing stream of consciousness in which objects fade in and out; James describes this as the "lingering of old objects" through which time is understood as "retrospective and prospective." ${ }^{39}$ This awareness of change defines the understanding of a thick duration through which time is understood as passage.

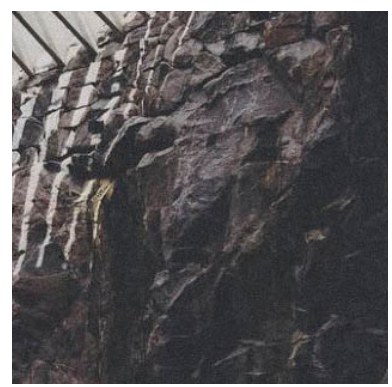


Notes

1 Sherover, The Human Experience of Time: The Development of its Philosophic Meaning, 225.

2 Ibid., 224.

3 Juhani Pallasmaa. "Inhabiting Time." Architectural Design 86 (2016): 53.

4 Günter Nitschke, From Shinto to Ando: Studies in Architectural Anthropology in Japan (London: Academy Editions, 1993), 33

5 Richard B. Pilgrim, "Intervals ("Ma") in Space and Time: Foundations for a Religio-Aesthetic Paradigm in Japan." History of Religions 25, no. 5 (1986): 256.

6 Ibid., 255.

7 Heidegger, "Building, Dwelling, Thinking," 152.

8 Ibid., 336.

9 Hans Ulrich Gumbrecht, Production of Presence: What Meaning Cannot Convey (Stanford: Stanford University Press, 2003), 151.

10 Kirk Warren Brown and Richard M. Ryan, "The Benefits of Being Present: Mindfulness and Its Role in Psychological Well-Being," Journal of Personality and Social Psychology 84, no. 4 (2003): 822.

11 Thera, The Power of Mindfulness, vii.

12 Gernot Böhme, "Atmosphere as Mindful Physical Presence in Space," Building Atmosphere, (2013): 27.

13 Thera, The Power of Mindfulness, 27.

14 Böhme, "Atmosphere as Mindful Physical Presence in Space," Building Atmosphere, 27. 
15 Sarah Robinson, Nesting: Body, Dwelling, Mind (Richmond: William Stout Publishers, 2011), 136.

16 Thera, The Power of Mindfulness, 33.

17 Brown and Ryan, "The Benefits of Being Present: Mindfulness and Its Role in Psychological WellBeing," Journal of Personality and Social Psychology, 823.

18 Ibid.

19 Böhme, "Atmosphere as Mindful Physical Presence in Space," 27.

20 Ibid.

21 Ibid.

22 Nitschke, From Shinto to Ando: Studies in Architectural Anthropology in Japan, 49.

23 Gernot Böhme, "Encountering Atmospheres a Reflection on the Concept of Atmosphere in the Work of Juhani Pallasmaa and Peter Zumthor," Building Atmosphere, (2014): 99.

24 Ibid., 97.

25 Gernot Böhme, "The Theory of Atmospheres and its Applications," Interstices: Journal of Architecture and Related Arts, vol. 15, eds. Ross Jenner and Andrew Douglas, trans. A.-Chr. Engels-Schwarzpaul (2014): 93.

26 Ibid., 94.

27 Steven Holl, Juhani Pallasmaa and Alberto Pérez Gómez, Questions of Perception: Phenomenology of Architecture (San Francisco: William Stout Publishers, 2006), 30. 
Hermann Schmitz, Rudolf Owen Müllan and Jan Slaby, "Emotions Outside the Box-the New Phenomenology of Feeling and Corporeality," Phenomenology and the Cognitive Sciences, 2011: 255.

Böhme, "Encountering Atmospheres a Reflection on the Concept of Atmosphere in the Work of Juhani Pallasmaa and Peter Zumthor," 97. Irwin Altman and Setha M. Low. Place Attachment, vol. 2, in Human Behavior and Environment: Advances in Theory and Research (New York: Plenum Press, 1992), 4

31 Paulo Providência, Architectonica Percepta: Texts and Images 1989-2015 (Zurich: Park Books, 2016), 165.

32 Juhani Pallasmaa, "Space, Place, Memory and Imagination: The Temporal Dimension of Existential Space," in Spatial Recall: Memory in Architecture and Landscape, ed. Marc Treib (New York: Routledge, 2009), 20.

33 Ibid., 30.

34 Henri Bergson, Matter and Memory (London: George Allen \& Unwin, 1970), 106

35 Ibid., 80

36 Pallasmaa, "Space, Place, Memory and Imagination: The Temporal Dimension of Existential Space," 25.

37 Henri Bergson, Matter and Memory, 125.

38 Sherover, The Human Experience of Time: The Development of its Philosophic Meaning, 371.

39 Ibid., 370. 


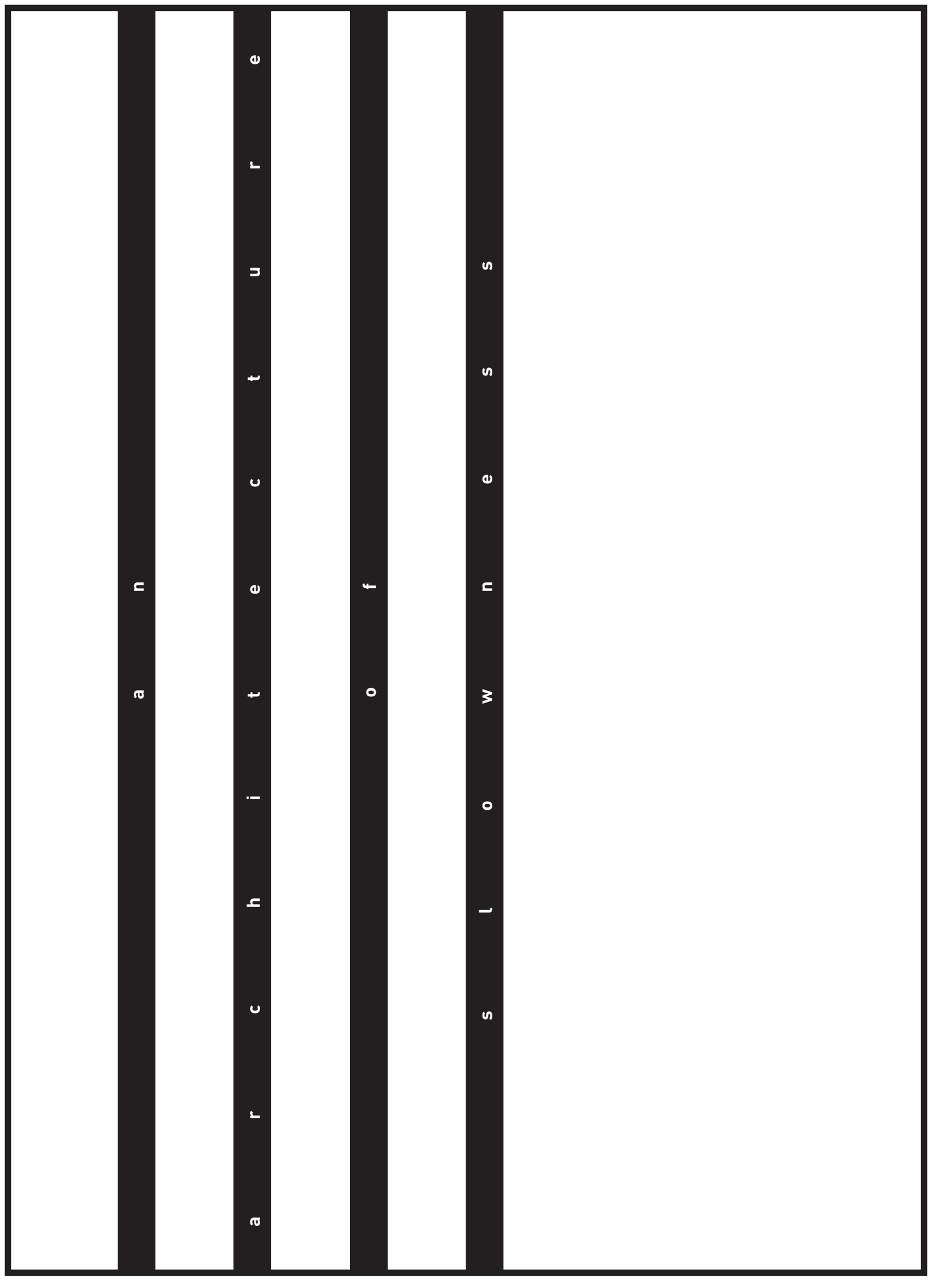


"The experience of a place or space is always a curious exchange; as / settle in a space, the space settles in me... We are in a constant exchange with our settings; simultaneously we internalize the setting and project our own bodies, or aspects of our body schemes, on the setting.

\section{Architecture as Vehicle for Inhabiting Time}

This chapter establishes the generators within an architecture of slowness.

\section{1}

Inhabiting time defines an architecture that manipulates spatial and perceptual elements to generate mindful physical presence within the mind and body. By investigating the sequential nature of the everyday, this thesis references the assembly of film as a tool for both the design and representation of spatial and perceptual collision.

Filmic assemblage is used to portray the diverse experience between spatial slits, or cuts, and axes, or dissolves. The previously discussed design research made apparent the varying levels of consciousness associated with the speed of movement: accelerated, slowed, and paused. Through perceptual density and spatial collision, a layered sequence of quickening and tumbles the inhabitant through varying levels of stimuli between moments of motion and pause.

This thesis considers the dwelling as the vehicle for exploring connectivity and its impact on mental and physical presence. The intent of this design research is to develop an architectural lexicon for slowness. 


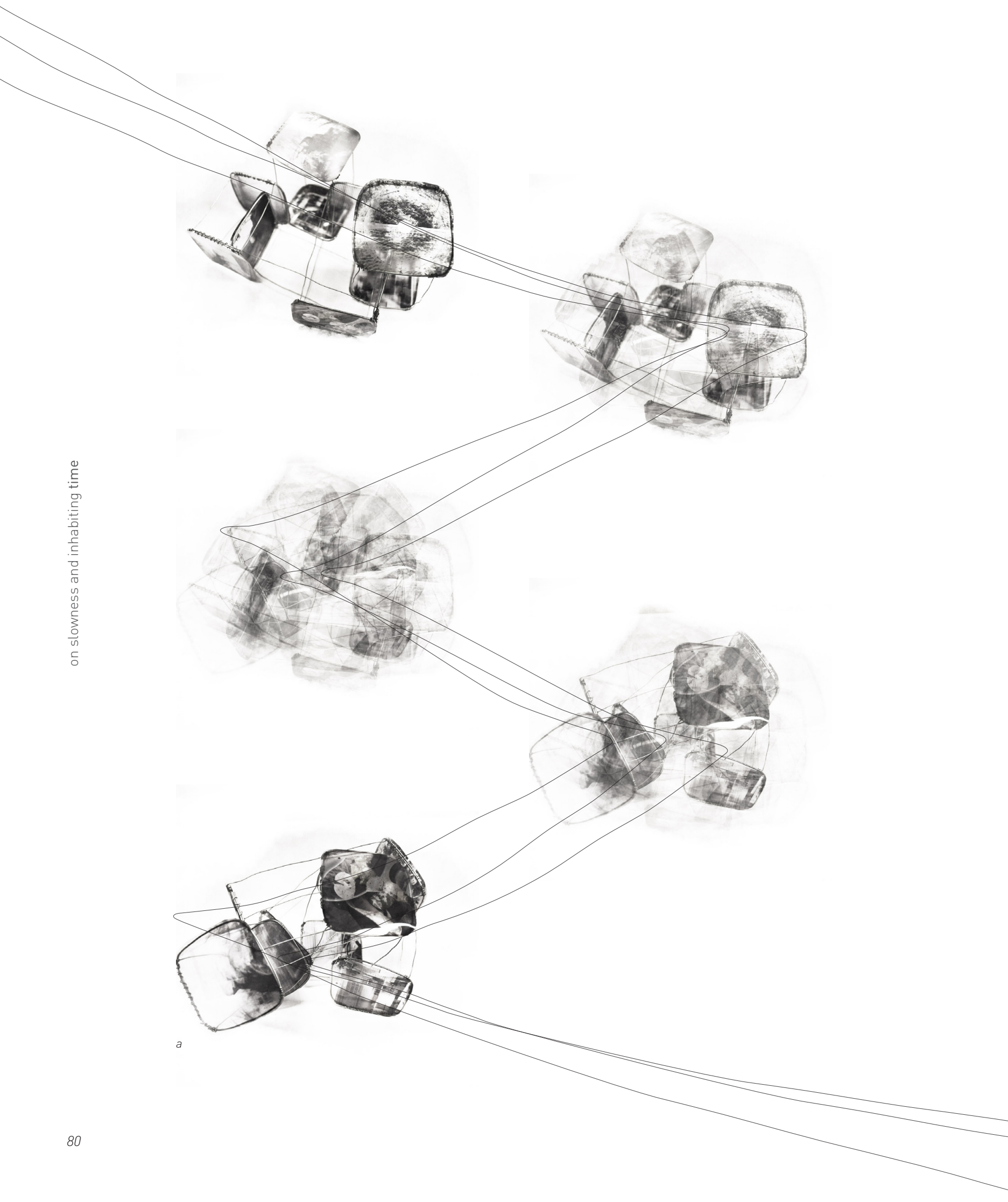




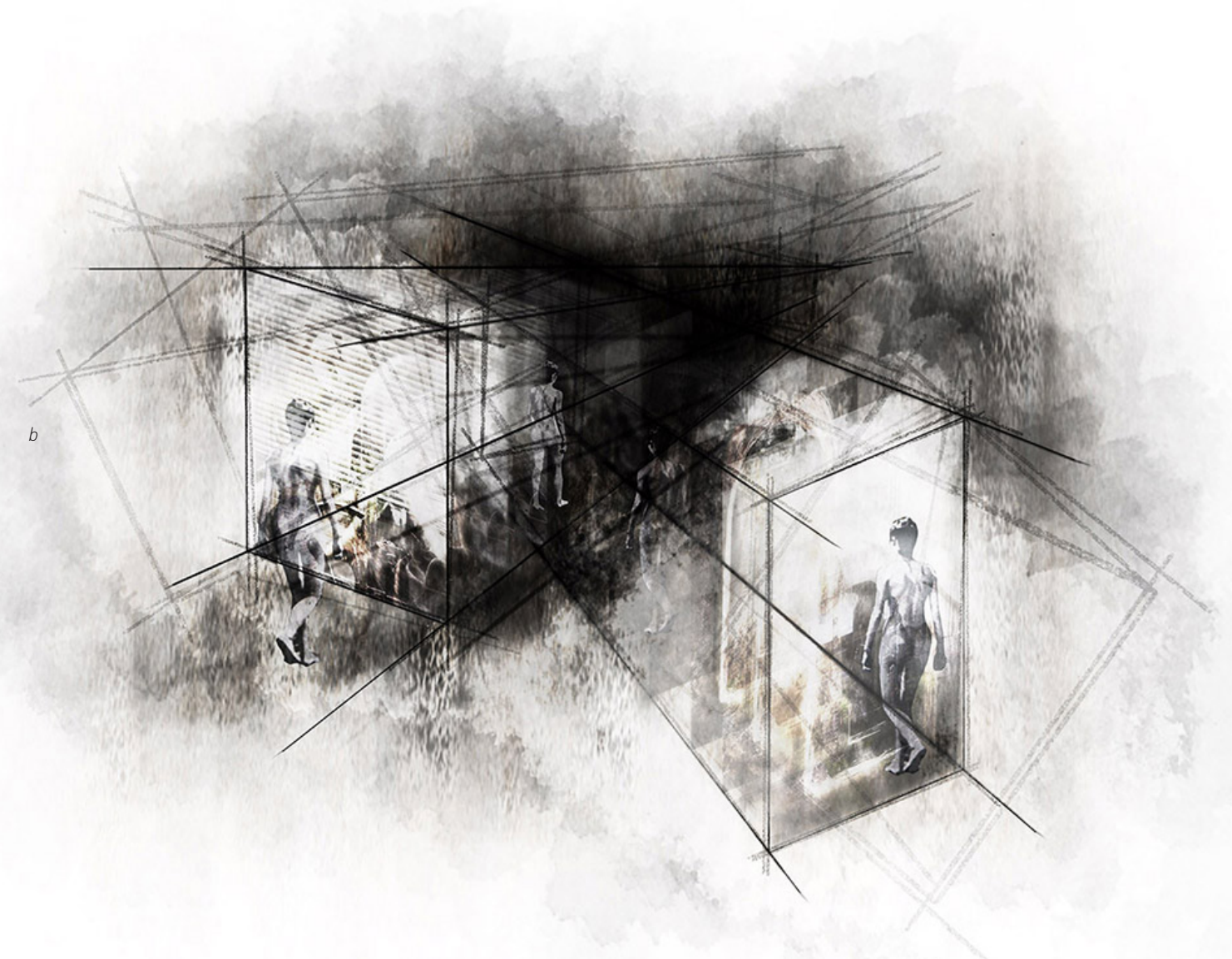

Montage Study

a_Wire Model; b_Experience of the Wire Model

Referencing Bergson's Circuit Diagram, the wire model separates present and memory images from the study titled, Everyday Overlay, into wire cubes that reflect spatial conditions. As the model is hit, the tumbling causes spaces to collide with one another; this collision is represented in the drawing above as a simultaneous motion through spatial and perceptual moments within the past, present, and future. 
The spatial and perceptual collision of two or more concurrent spaces, also defined as thresholds of juxtaposition, generates an emotive response that begins to presence the mind and body. Memorable experiences develop when a dialogue forms between perceiver and perceived or subject and object. The intangible and sensuous elements, identified as atmospheric generators, contain specific characteristics that produce these experiences. As previously discussed, Pallasmaa considers these generators as, "qualities of matter, space and scale [which are] measured by the eye, ear, nose, skin, tongue, skeleton and muscle." 1 The experience of space is relative to the relationship amongst spatial and atmospheric generators which l've defined as generators of slowness.

Generators of Slowness act as a tool for creating moments of juxtaposition within viscous procession of frenetic and slowed variance. The unfolding of spatial and atmospheric constructs was developed from my travels in Japan wherein I experienced both contemporary and traditional Japanese architecture, temples, and gardens. Within this thesis, the specific generators of slowness used to develop temporal inhabitance include: proportion and scale, rhythm, light and shadow, sight, and materiality. The descriptions below briefly introduce the Generators of Slowness while the following case studies can be found in Appendix A from Japan further define the generators.

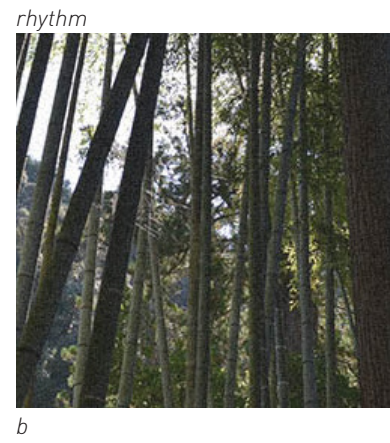



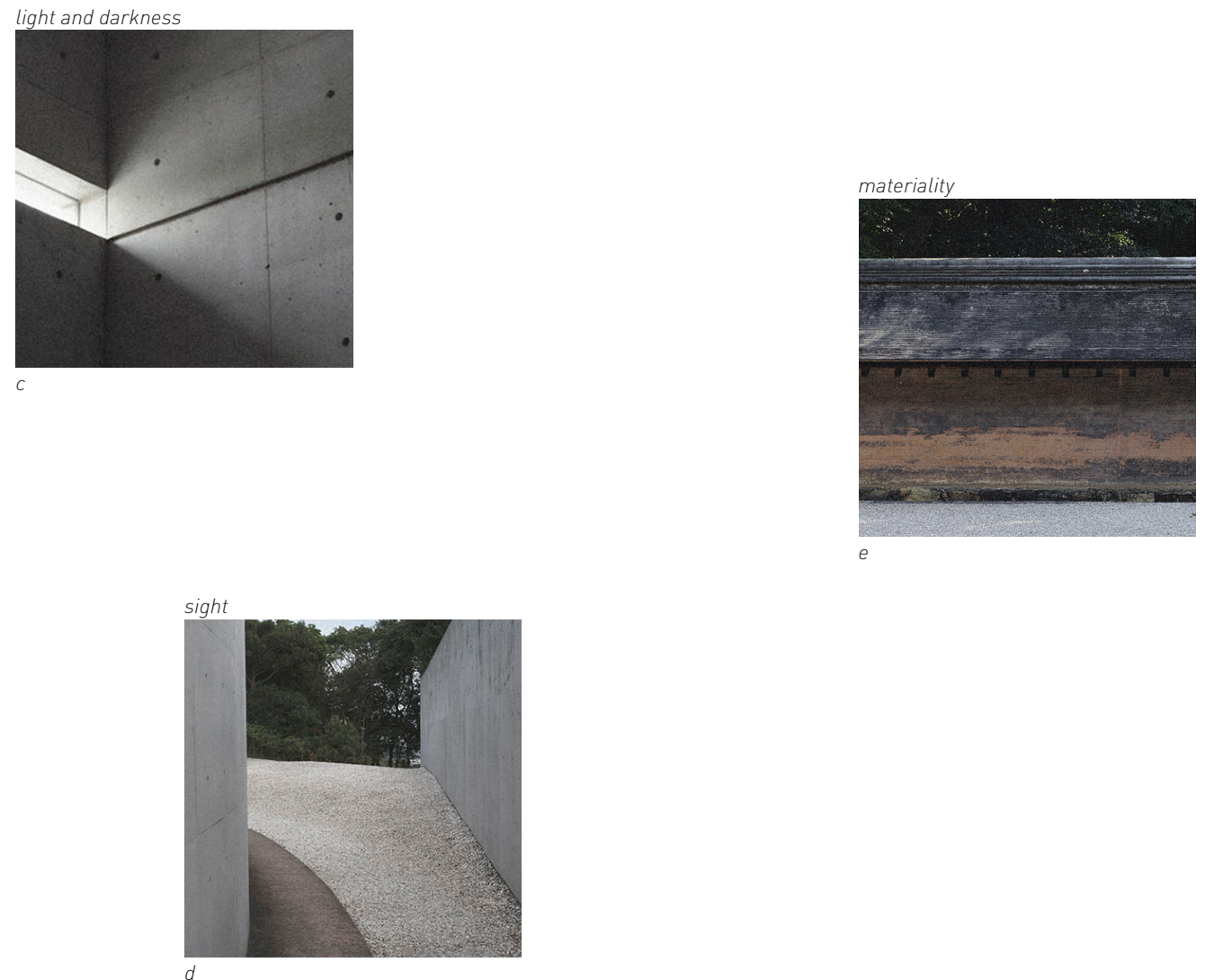
Spatial proportions create distinct moments of contrast that vary one's presence throughout an experience. These proportional variances can occur in plan or section and are characterized by the compression and expansion of space. Compression pushes individuals through space whereas expansion denotes rest; in other words, compression defines spaces of motion and expansion defines spaces of stillness. Moving through compressed to expanded spaces makes one aware of both oneself and the space being inhabited. Such that, proportion and scale use variance to emphasize moments of pause within the everyday freneticism.

Rhythm is defined as the repetitive organization of elements and spaces. As such, the rhythm of and within architectural frames occur at regular or irregular intervals and establishes varying densities within an experience. This develops an intertwined relationship between perceiver and perceived or subject and object whereby the sequential revealing of layers is followed with the eyes and experienced by the body. Within natural and man-made environments varying rhythms create distinct slices in space and time that correlate with the rhythm of the eyes and body.

The ethereal and temporal characteristics of natural light, including its direction and intensity, influence the atmosphere by orchestrating spatial and perceptual variances. These variances juxtapose light with dark to create moments of pause through a felt compression and expansion of space and time. While guiding the mind and body through a procession, these thresholds of juxtaposition heighten suspense and anticipation 
in order to evoke a specific mood. Although light and shadow are both influential generators, their influence on the other generators is most significant when considering the vicious experience of architecture.

For perception, what, how, and when things are revealed is intrinsic to its interpretation. By concealing and selectively revealing a spatial and perceptual unfolding translates into an elongated reading of a procession. This elongation further heightens awareness of the mind and body in space as well as the space itself. By doing so, the slow procession allows for sustained attention whereby slow looking, a prolonged viewing of a thing that brings attention to its intricacies, can shift attention inwards from the everyday freneticism.

Materials are experienced by way of tactility, both as texture with the eyes and touch with the body, and a multiplicity of layers that are both physically and cognitively presencing. They slow one's procession physically through tactility, shape, size, and type; and cognitively, through temporal layers including the patina, intricacies, and type. The specific tactile and felt qualities of materials are elevated when in contact with the various other generators of slowness mentioned above. In this sense, the interplay between form and the various generators create the backdrop for enmeshed experience of space and time. 
5.3.1 Physis is the Greek philosophical term for nature; it is defined by the hours of the day, seasons of the year, altitude, latitude, and longitude. ${ }^{2}$ This thesis considers the atmospheric qualities of place, unbound from the isotropic definition of space, as a tool for guiding the mind and body towards presence. The four elements below, sound, smell, light and shadow, and sight, outline the felt atmospheric qualities of the coast.

\section{Of Smell}

As I made my way towards the coast I was surprised by the intensity of ocean air. Although I was approximately two block from the coast I could feel crispness of the air on my skin. I had forgotten how fresh the air smelled near the ocean. It was the first clear day in weeks and the smell of rain still lingered in the air. It was cooler than in the city and I decided to put on my raincoat to break the wind. The closer I got to the water, the more intense the smell became. Finally, I was standing at the edge of the ocean, as the wind blew through my hair, breathing in the crisp ocean air. There was no singular memory which came to mind as I stood on the beach, rather a collection of several memories spent watching the waves crash into the rocks in my youth. The layers of time began to overlap one another as I stood watching time unfold.

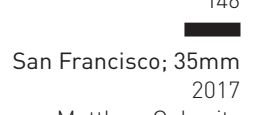

Matthew Gelowitz

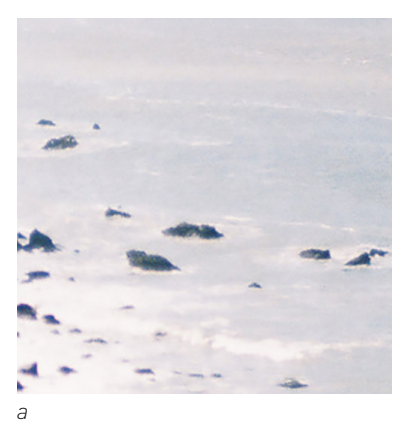




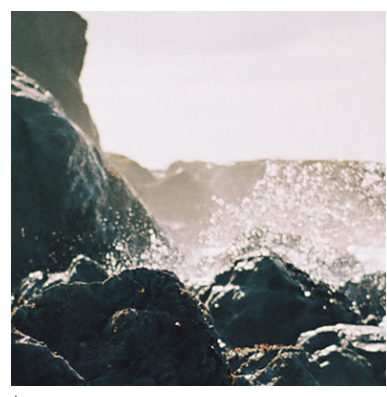

Of Sound

I stood on the edge of the cliff with my eyes closed, listening. I heard the silence of the coast. Not an absolute silence, rather a low hum from the water, boats, and people. It contrasted the everyday noise and traffic not too far from this place. I stood listening for what seemed hours to the murmur of the waves. Letting it settle in me and me submersing in it. I became mindful of the water crashing against the rocks. The people laughing as they made their way down the forested trail. I was conscious of a low whine in the background, unsure if it came from the boats or a lighthouse nearby; barely noticeable but consistent. The rhythmic passage of time, was felt in the silence of the coast, and in that moment, I became the place. 


\section{Of Light and Shadow}

It was near sunset when I spent several hours walking the sea edge. I stood on the edge of the cliff watching the light and its shadows shifting on the landscape. To the west, the sun beamed down on the water crashing against the rocks, creating a haze around a man walking along the shore. In the east, it glistened on the landscape and Golden Gate bridge. As I stood at the edge of the cliff, I noticed the atmosphere changing. I looked to the west to see a dark cloud rolling in. Slowly, the light vanished and with it the shadows; in its place came darkness. I stood for some time, watching the storm roll in, feeling droplets of rain on my face and hair. I looked across the ocean and could no longer see the landscape in the distance. As the darkness washed over me, I became mindful of the natural ability for light

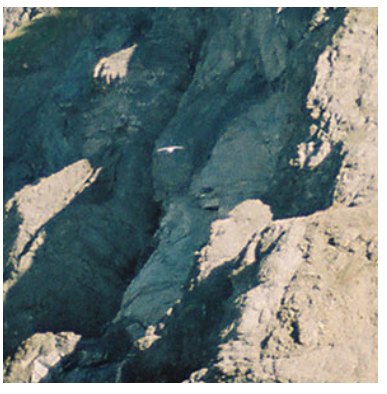
to orchestrate an intense temporal passage. 


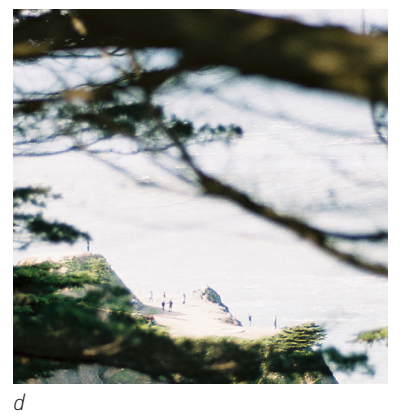

Of Sight

I made my way towards the edge of the coast, looking around, watching it unfold. The forested cliff allowed only glimpses to the water and landscape in the distance. I moved past some people as I made my way towards the edge of the cliff; layers of the view unfolded through the trees like a cinematic montage. The closer I got to the cliff, the more of the view I saw, I became mindful of the intensity of the waves as they crashed against the beach. In the distance, I could see a group of surfers and wondered how cold the water was at this time of year. Finally, I was standing at the edge of the cliff, watching the waves rolling in and out of the beach; there was always something so silencing about the water. I felt my presence in it and although I lost track of time, as a number, I was very aware of its passage. 


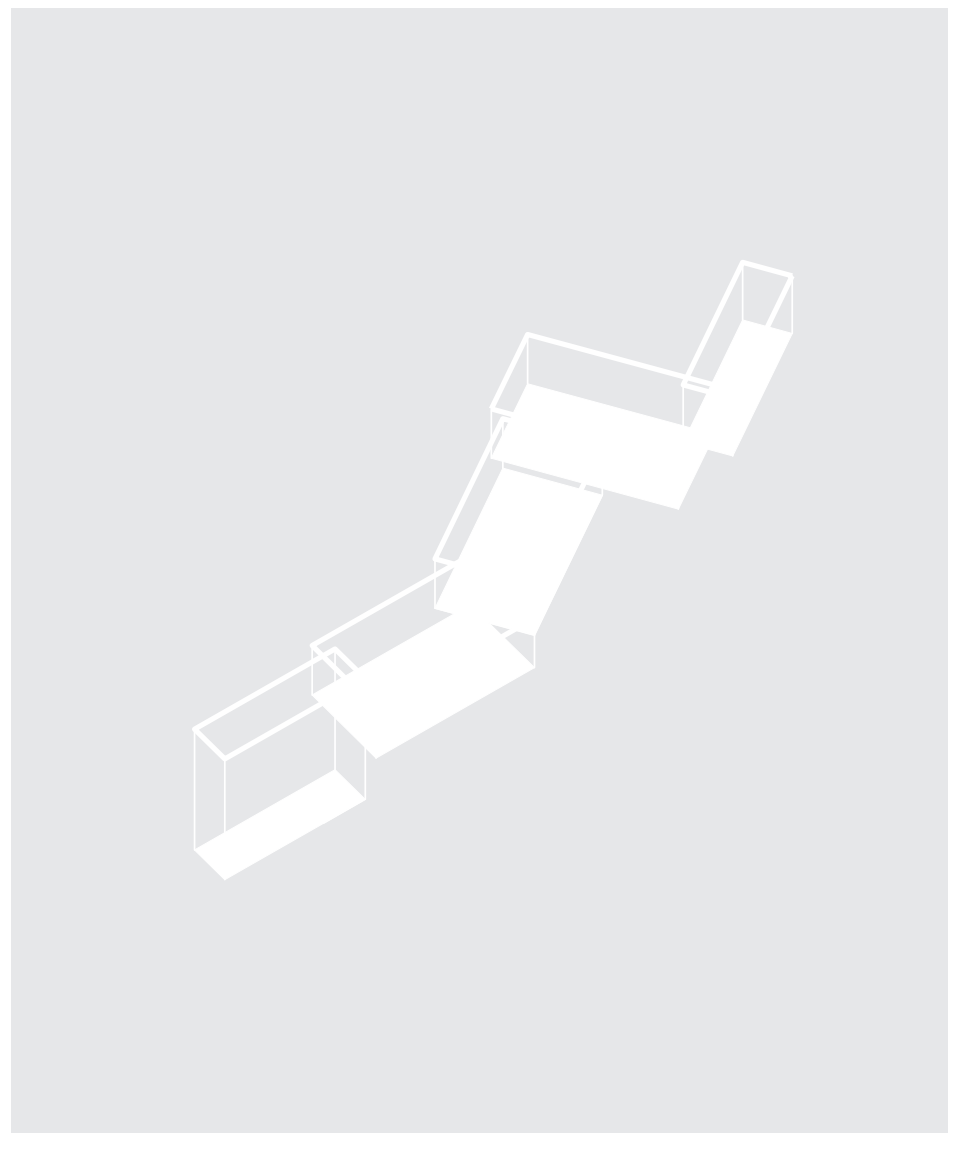

147

Sequence 0

Both sequences of the dwelling presented in this thesis are set on an abstracted site on the northern coast of San Francisco, California. The project is situated on a moderately forested terrain, on a plateau which drops considerably to overlook the Pacific Ocean. This specific setting was chosen as it embodies the junction between city and nature, built and unbuilt, frenetic and slow. This dramatic landscape acts as the unfolding object in the inhabitant's everyday. Specifically for Sequence 01, concealing and selectively revealing the landscape (sky, trees, and water) emerges a dialogue between subject and object within a choreographed silence. 


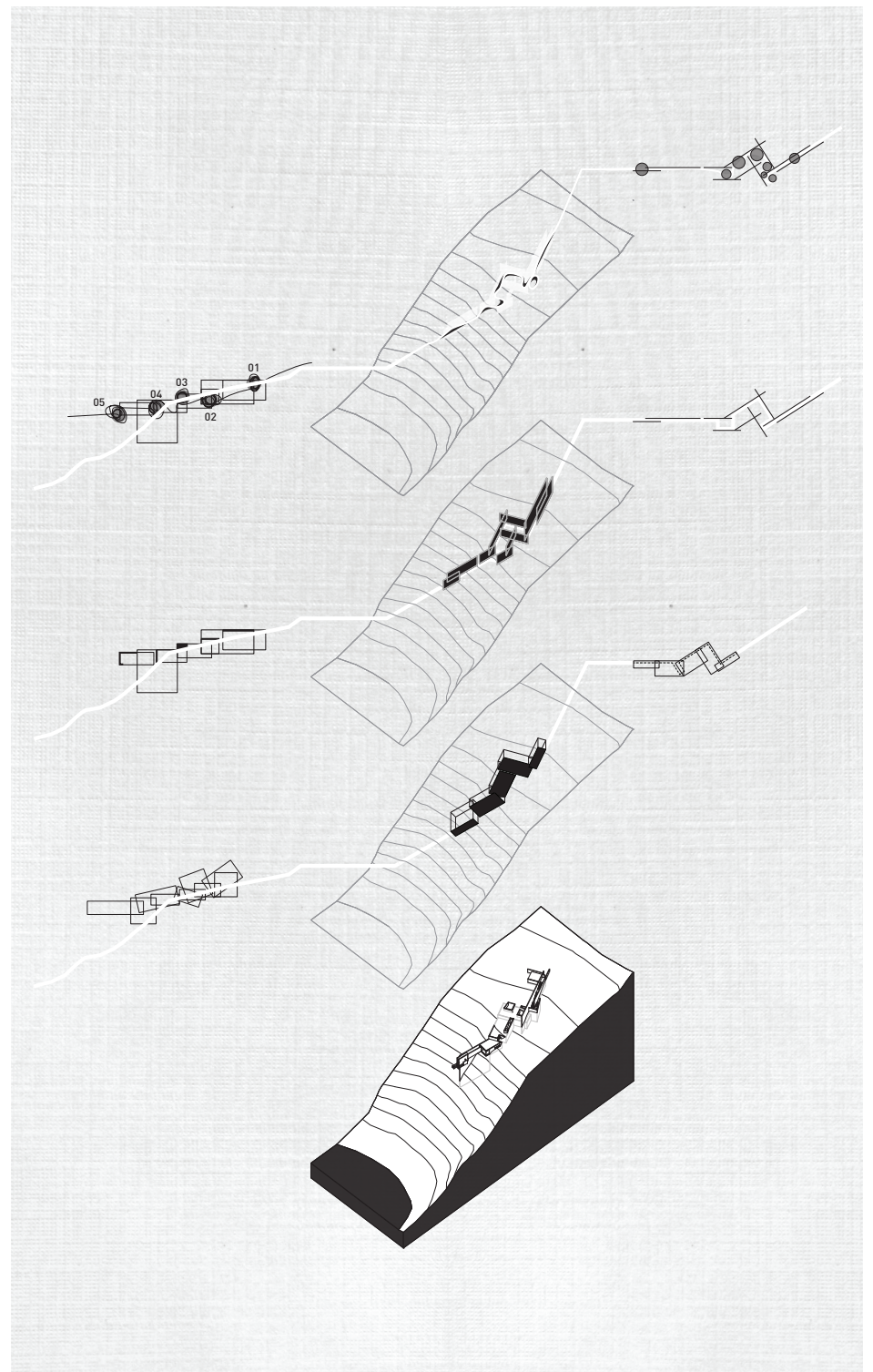

$\mathrm{f} 48$

Theory to Design; Sequence 01

The concept of tumbling was harnessed in the dwelling as a way of moving both down and across the landscape. From tumbling, spatial and planar collision were explored in guiding the individual through an unfolding architecture. In the sectional representation of this exploration, the thick dark lines represent moments of unfolding within the procession. Through thresholds of juxtaposition, the unfolding architecture creates moments of pause or expansion that are defined as a thickened experience of space and time. 
proportion
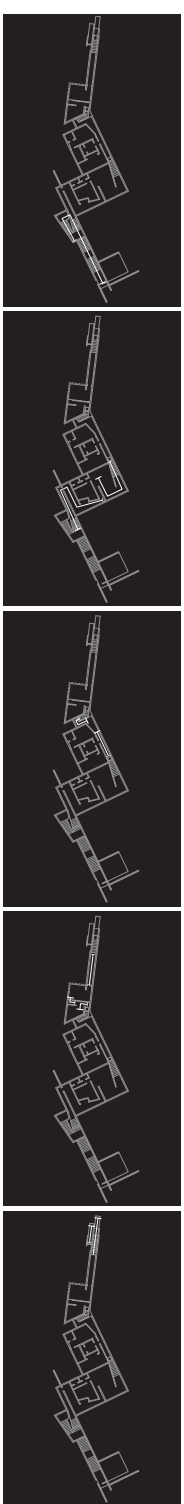

f49

Generators of Slowness; Sequence 01
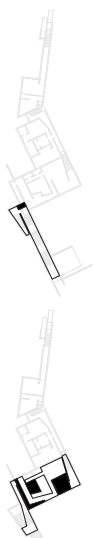

(

it

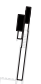

illuminance

movement

material

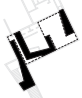

害
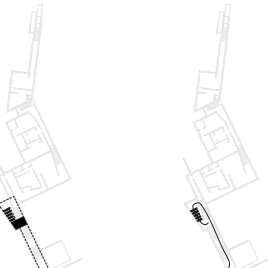

$b$
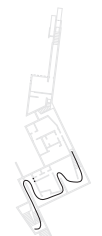

1

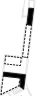

1
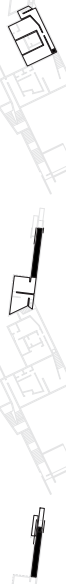

By separating and distinctly representing each generator by level, the study uncovers how each generator acts on its own and with each other. Within the investigation, proportion uncovers compression and expansion; window uncovers sight both vertically and horizontally; illuminate highlights light and dark moments; movement represents the passage and rhythmic elements within the procession; and materiality shows the different materials experienced by the feet and the walls. 


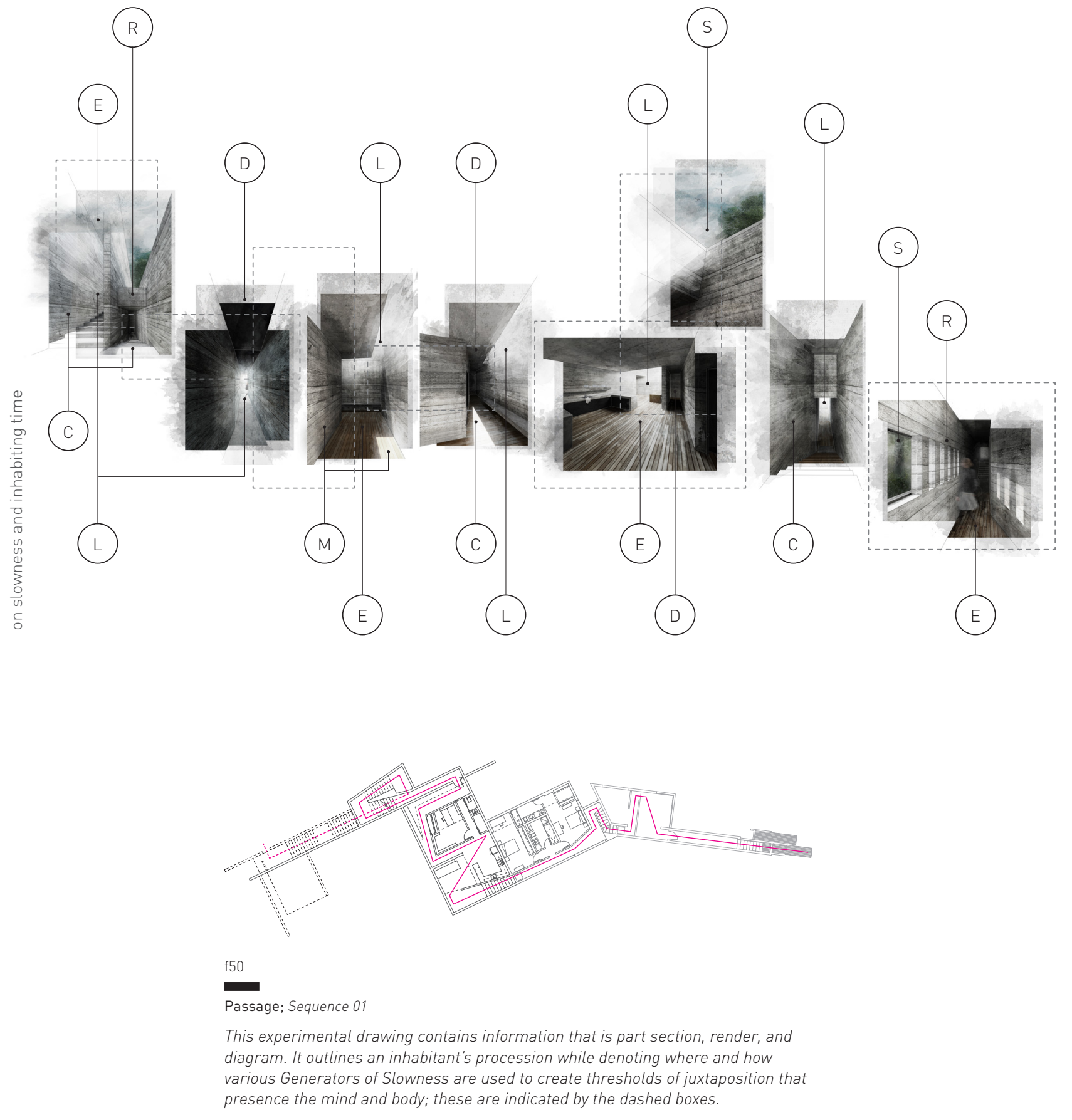




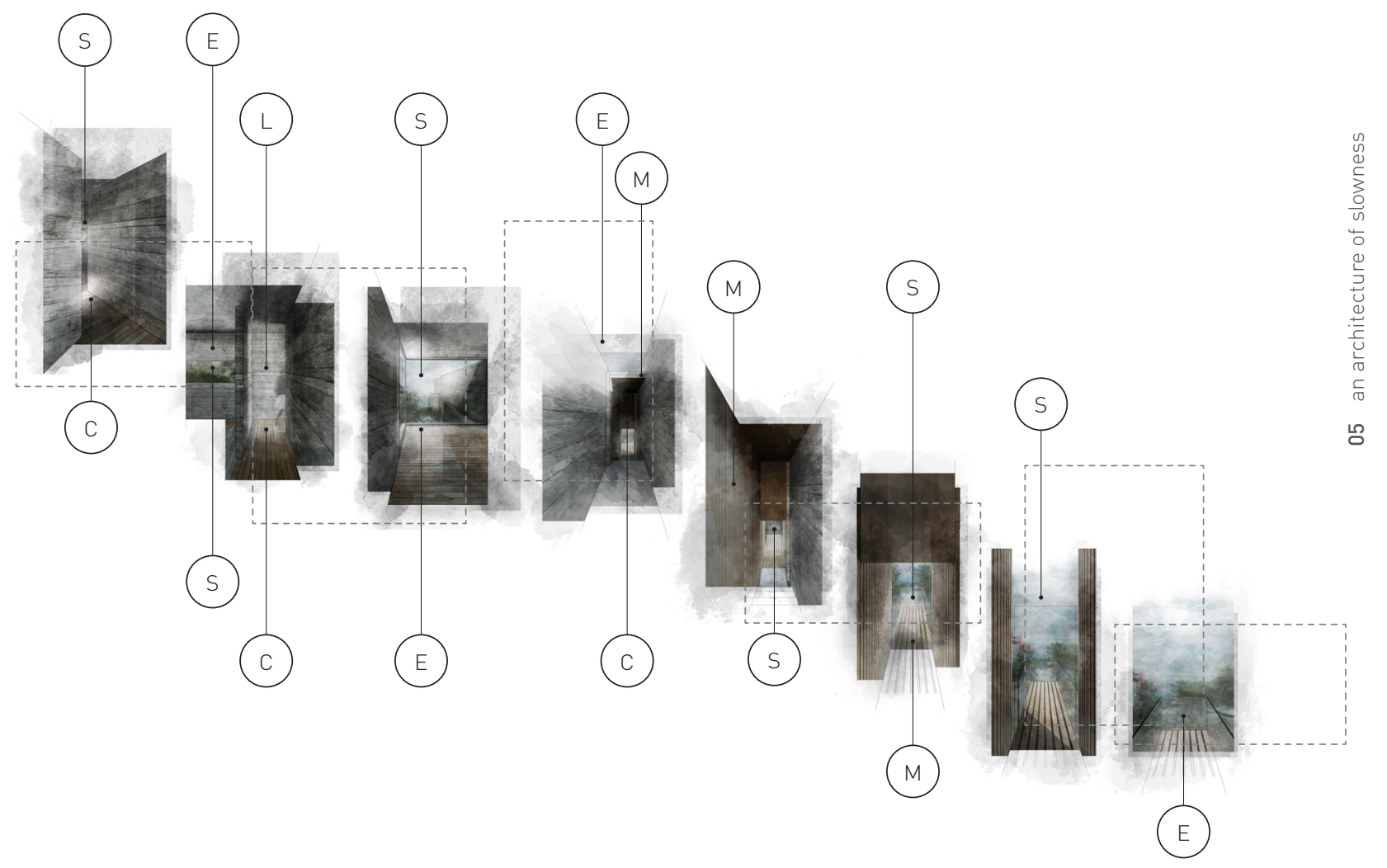

C compression Expansion $\mathrm{R}$ rhythm L light $\mathrm{D}$ darkness $\mathrm{S}$ sight $\mathrm{M}$ material

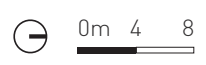




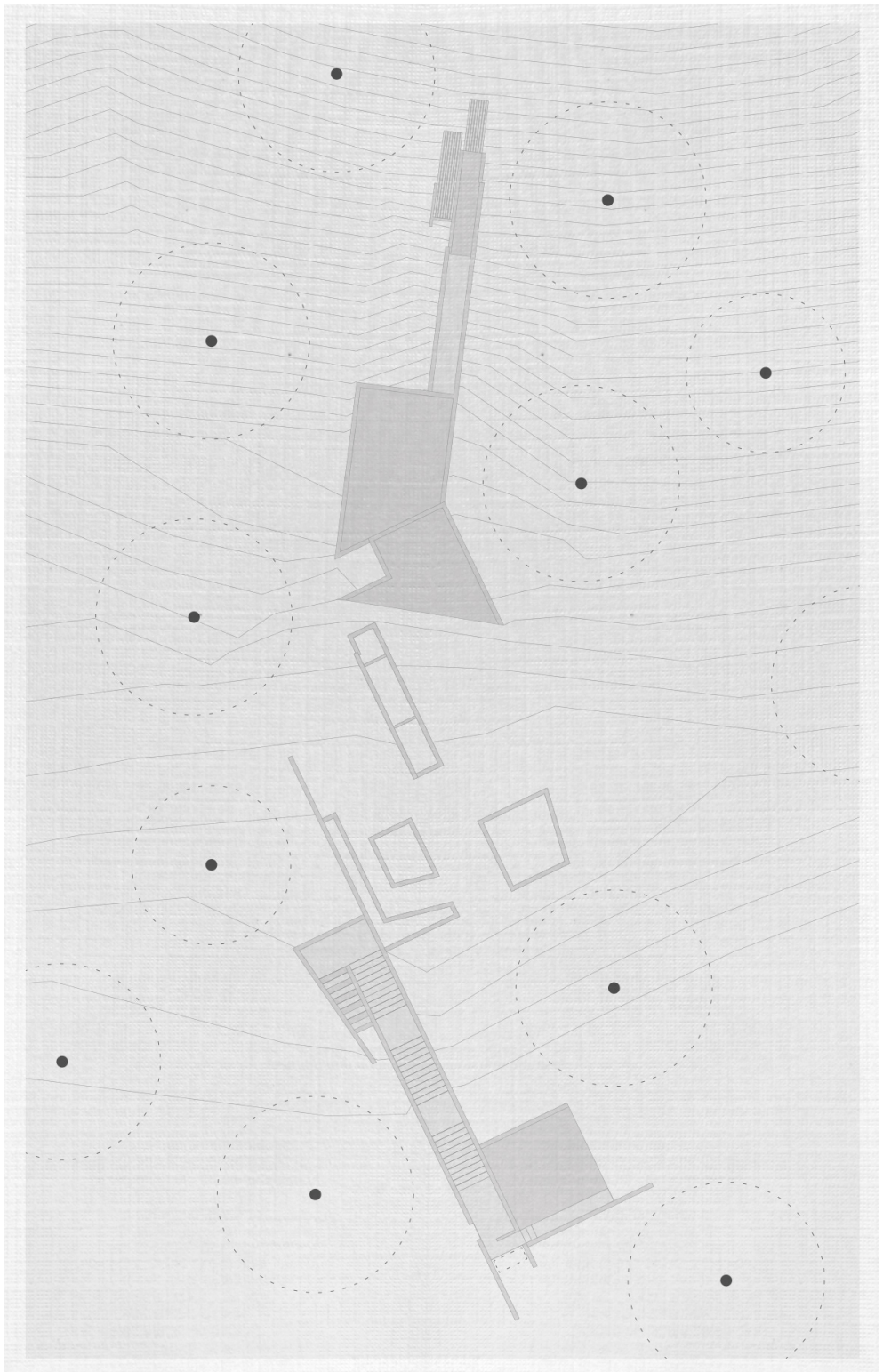

f51

Orthographic Drawings

a_Roof, Sequence 01

The dwelling is angled to the West in order to inhibit direct views to the Pacific Ocean until the final rooms of the house reveal it; this introduces the concept of concealing and selectively revealing elements of the landscape through procession, including the sky, terrain, and water. As the inhabitant progresses towards the entrance glimpses of the ocean are revealed between the trees. 


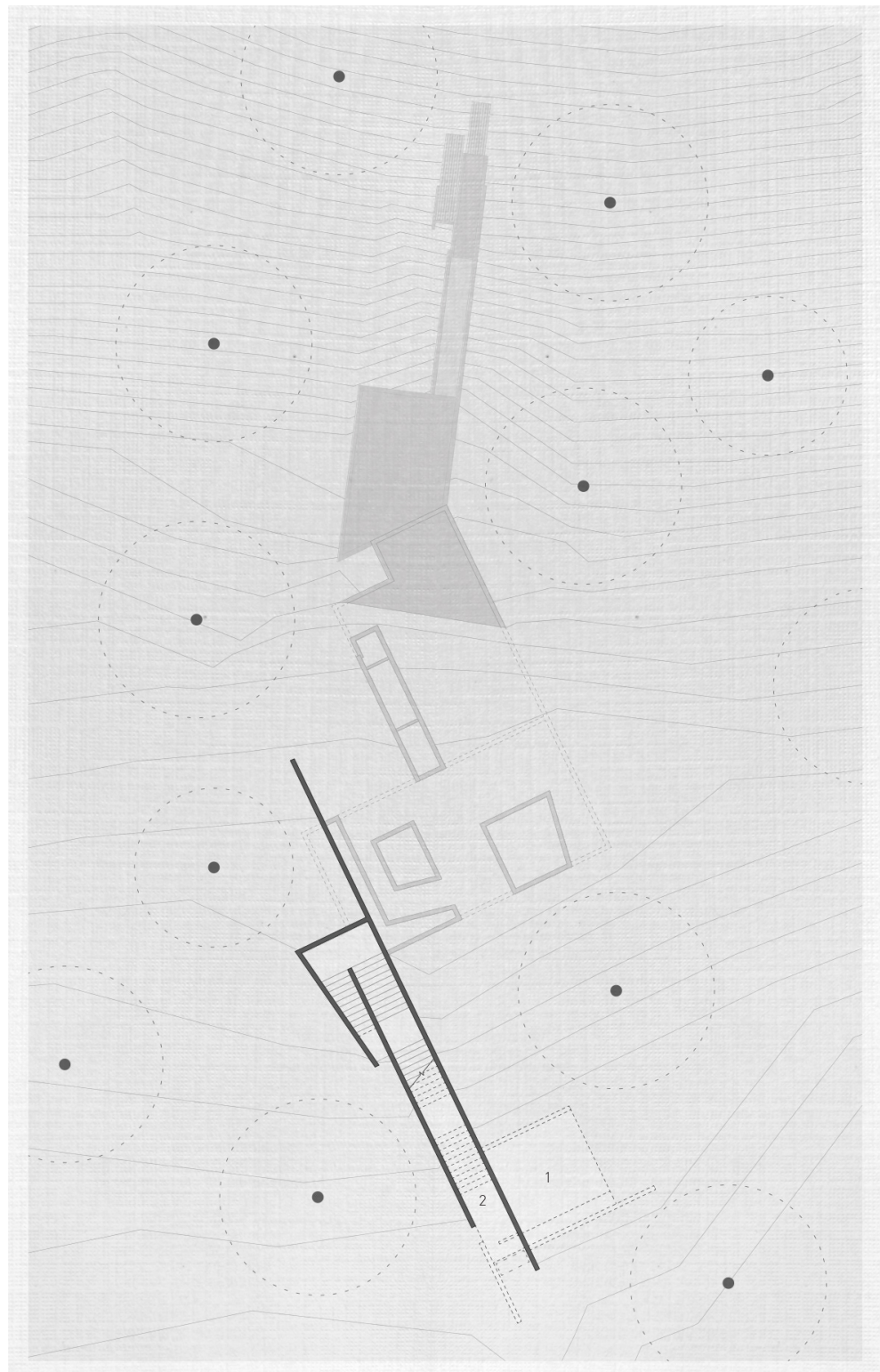

Orthographic Drawings

b_Level 01, Sequence 01

1 carport 2 entrance stairs

(1) $0 \mathrm{~m} \quad 4 \quad 8$ 


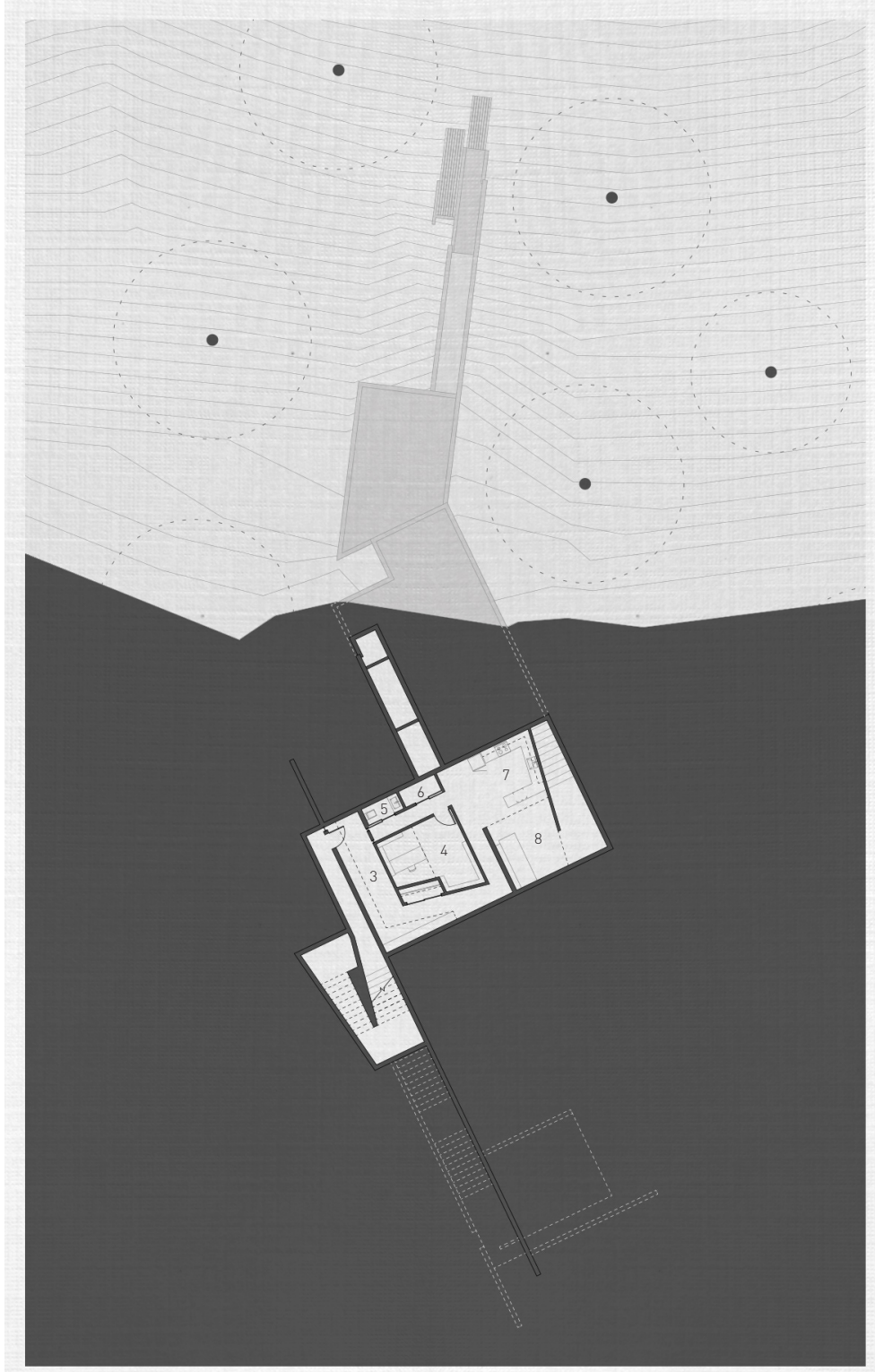

f51

Orthographic Drawings

c_Level 02, Sequence 01

3 foyer 4 study 5 powder room 6 pantry 7 kitchen 8 dining room 


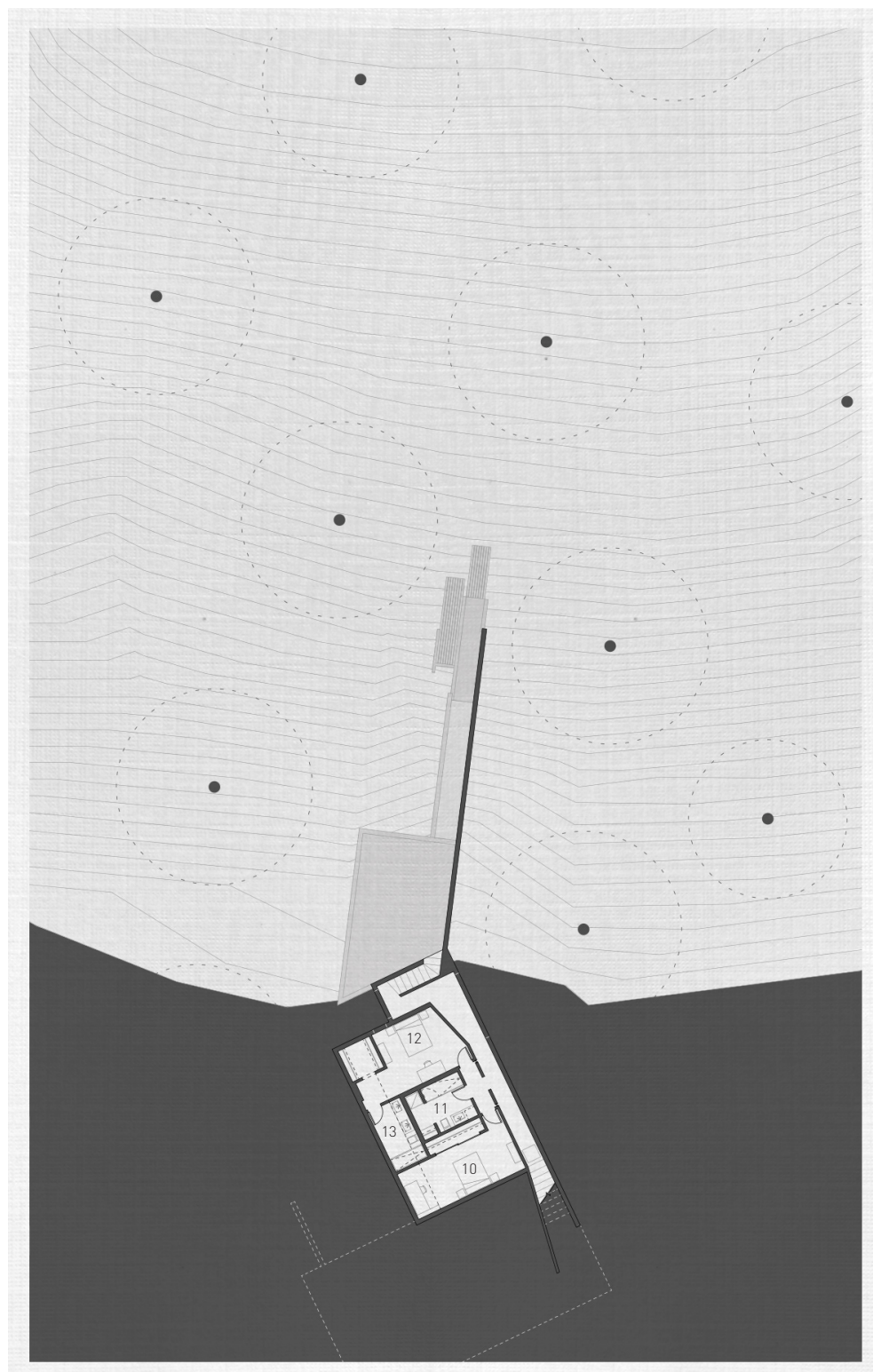

f51

Orthographic Drawings

d_Level 03, Sequence 01

10 guest bedroom 11 main bathroom 12 master bedroom 13 ensuite

(1) $0 \mathrm{~m} \quad 4 \quad 8$ 


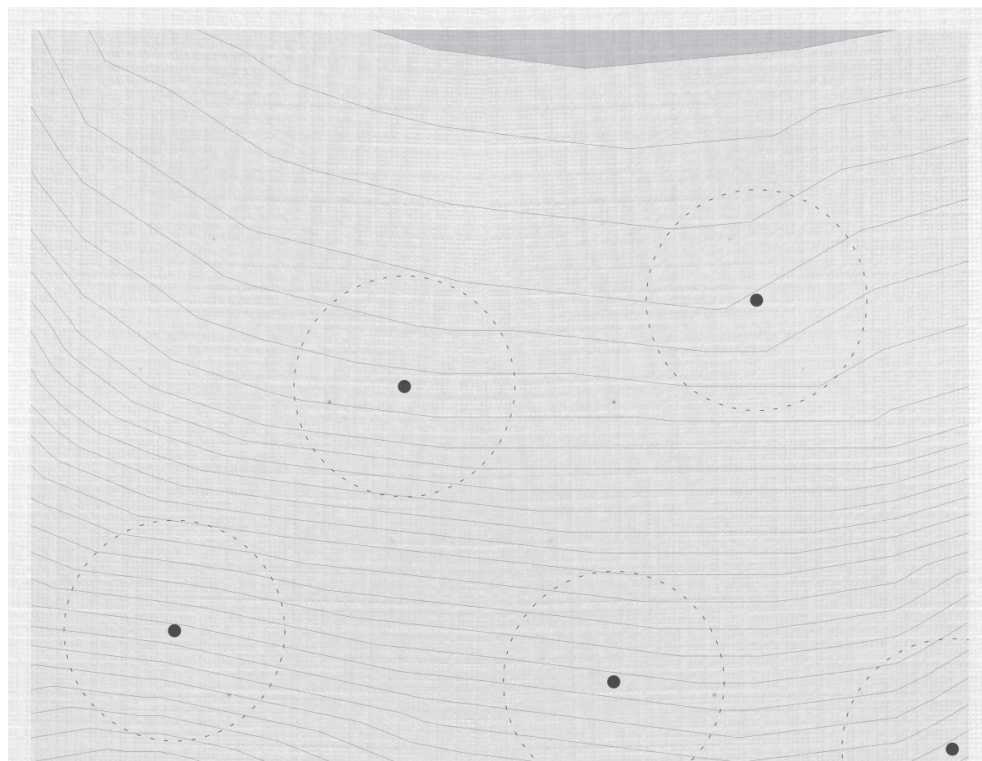

$$
\text { f51 }
$$

Orthographic Drawings

e_Level 04, Sequence 01

14 living space 15 space of silence 


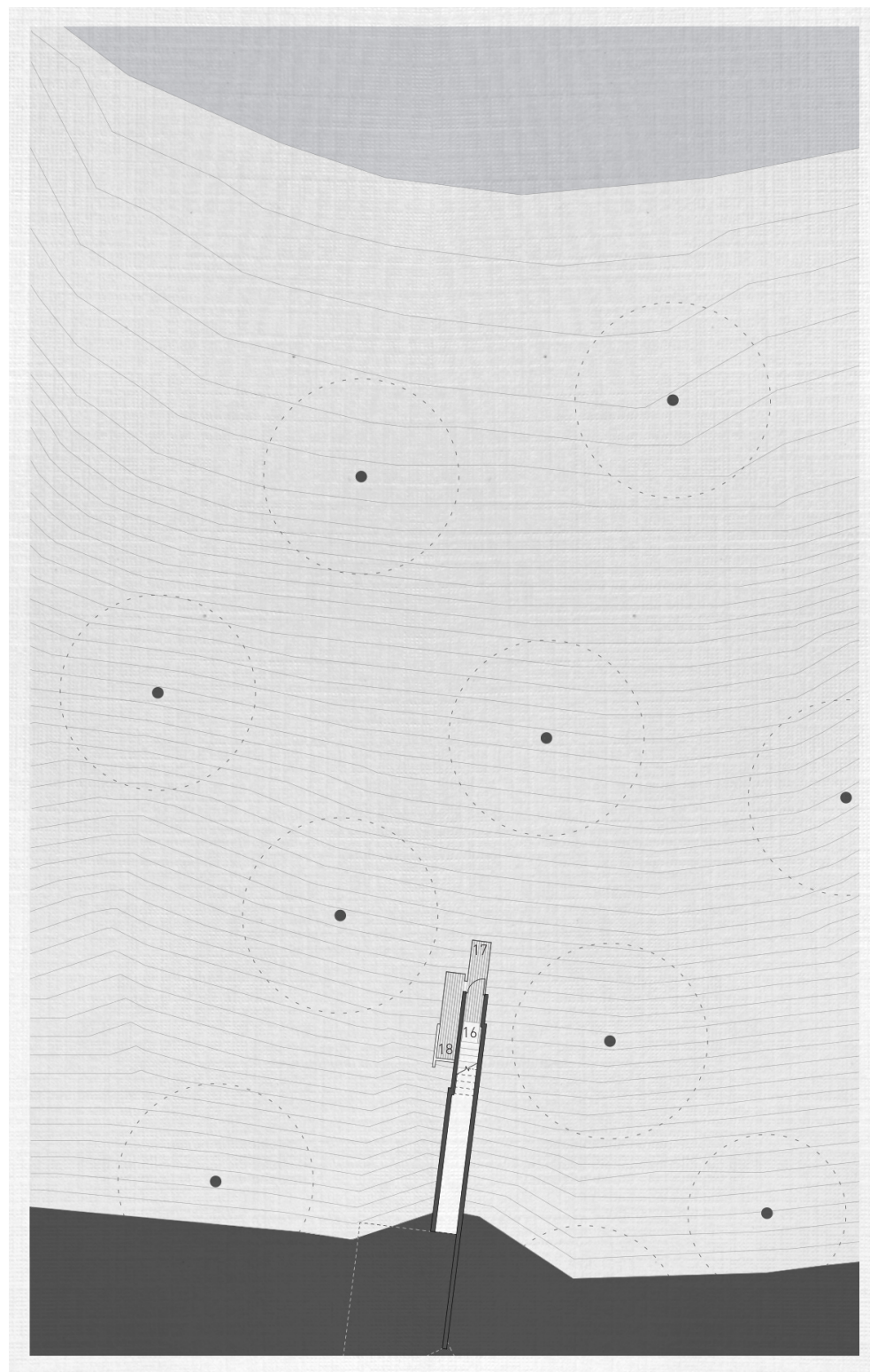

f51

Orthographic Drawings

f_Level 05, Sequence 01

16 space of silence_seen 17 space of silence_seen, outside

18 space of silence_hidden, outside

(1) $0 \mathrm{~m} \quad 4 \quad 8$ 

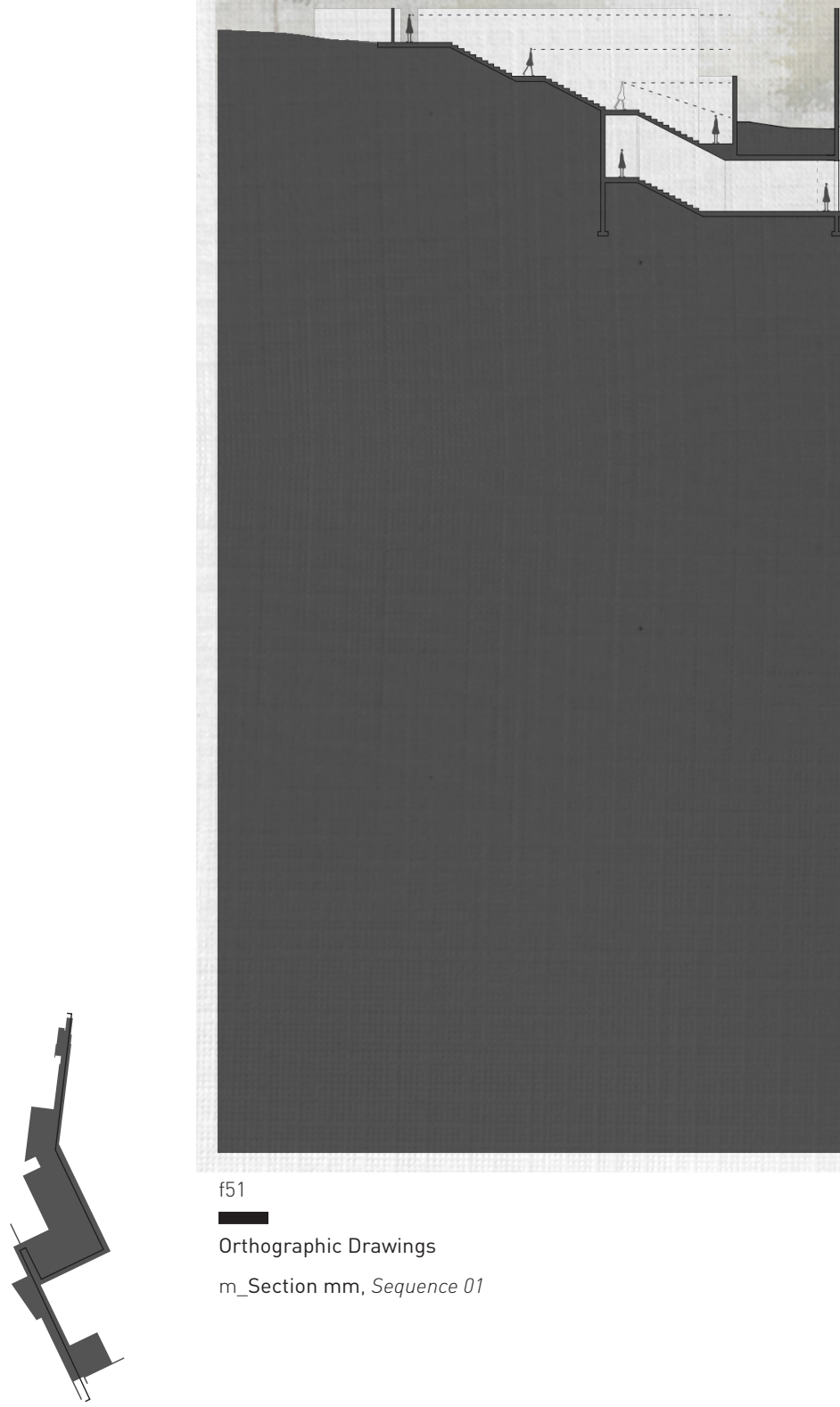

\section{f51}

Orthographic Drawings

m_Section mm, Sequence 01 
(1) $0 \mathrm{~m} \quad 4 \quad 8$ 


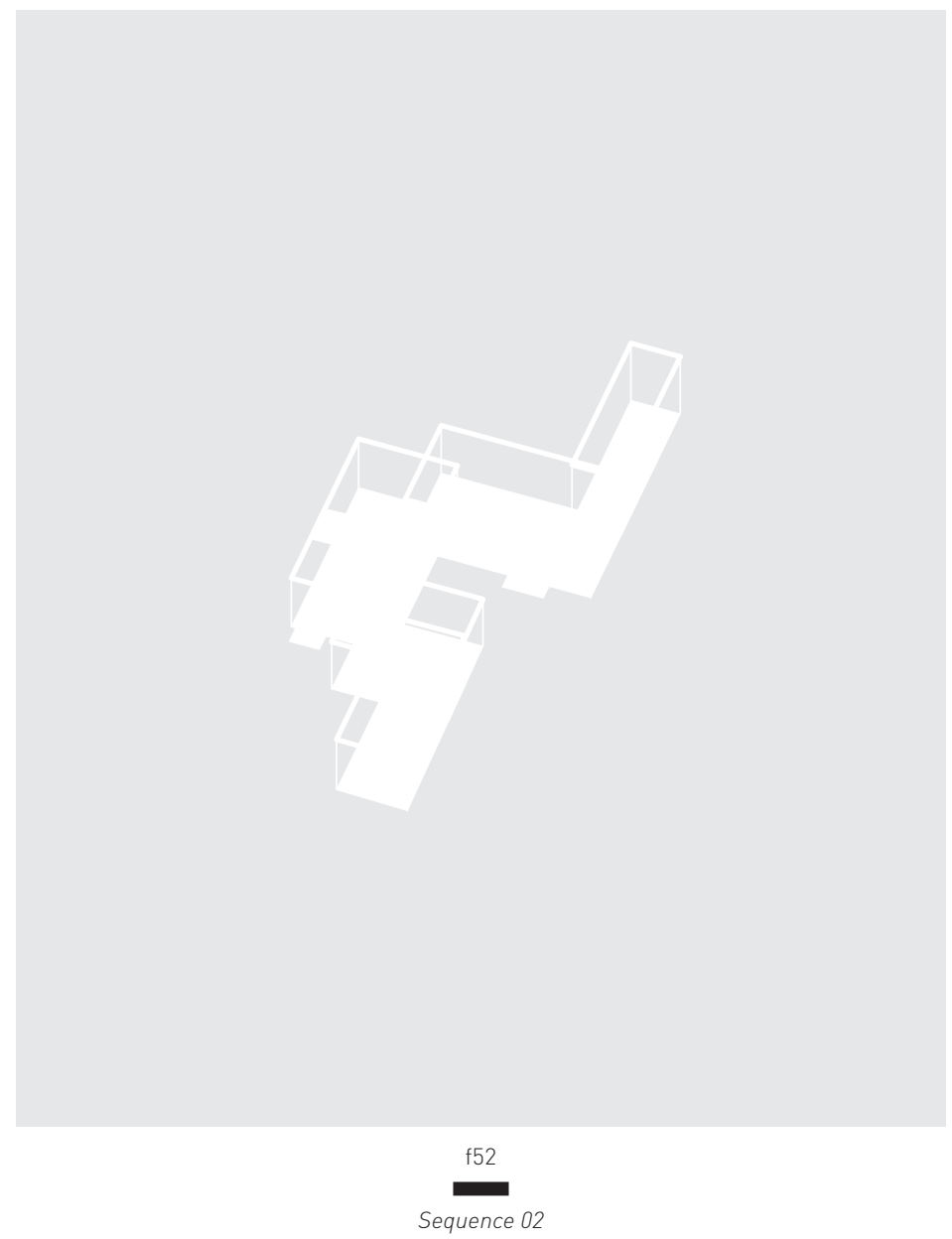

The second sequence introduces moments of quickening through perceptual density and spatial collision in order to heighten the felt experience of slowness within the dwelling. Cinematic assembly is used as a tool for developing and distinguishing between cuts and dissolves. In doing so, spaces squeeze inward and outward, perspectives are overlaid, moments are expanded, and time is shared to create an architecture of slowness. 


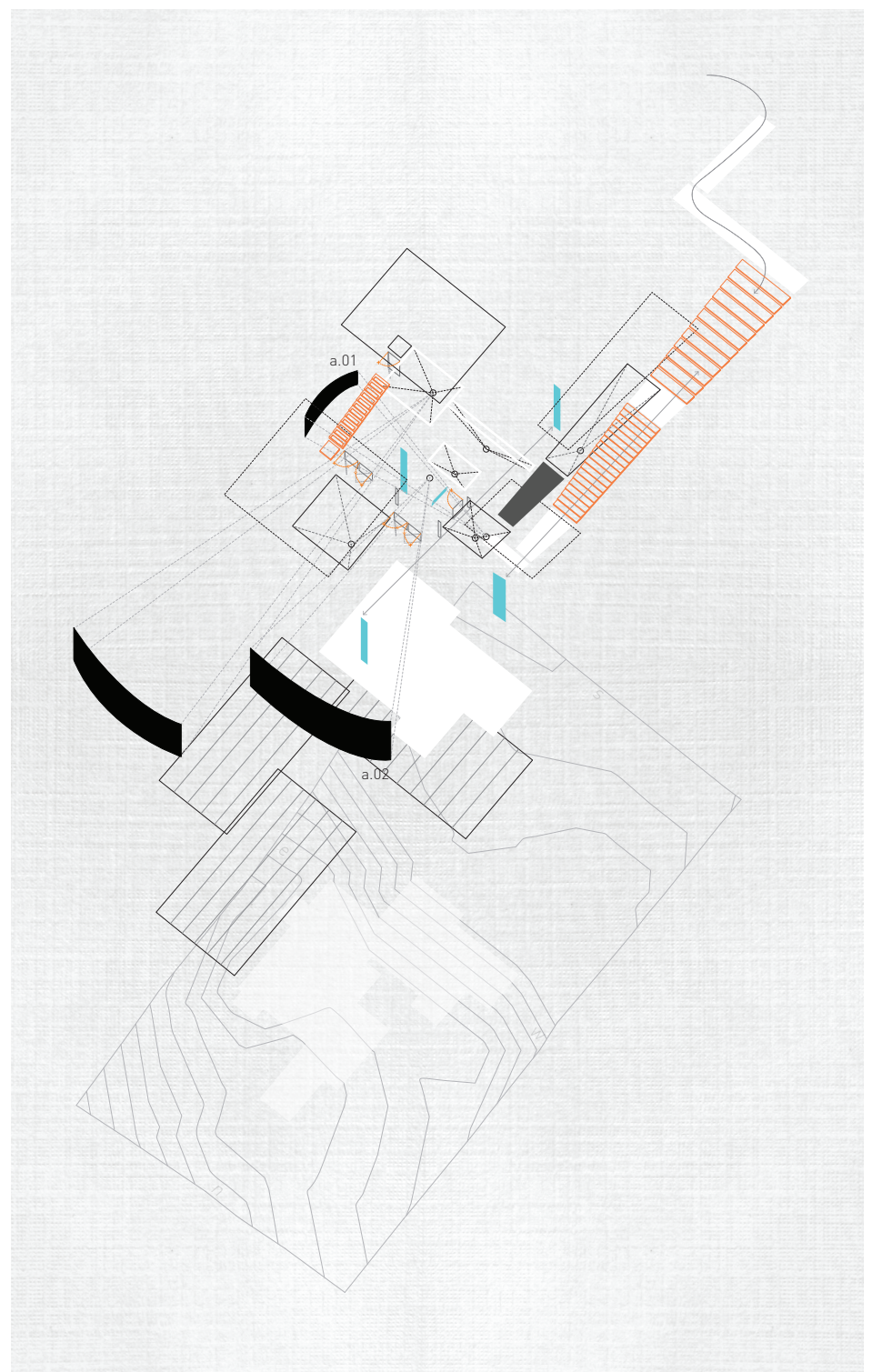

f53

Spatial and Perceptual Collision

a_Level 01 - Level 02, Sequence 02

The concept diagram, Spatial and Perceptual Collision, references the filmic assembly of various film directors in order to either condense or expand space and time. The montagist approach of Eisenstein and Marker was referenced to create rhythm, both regularly and irregularly, in order to conceal and selectively reveal various elements of the dwelling. These cuts heighten anticipation and suspense while representing compressed moments within the procession. 


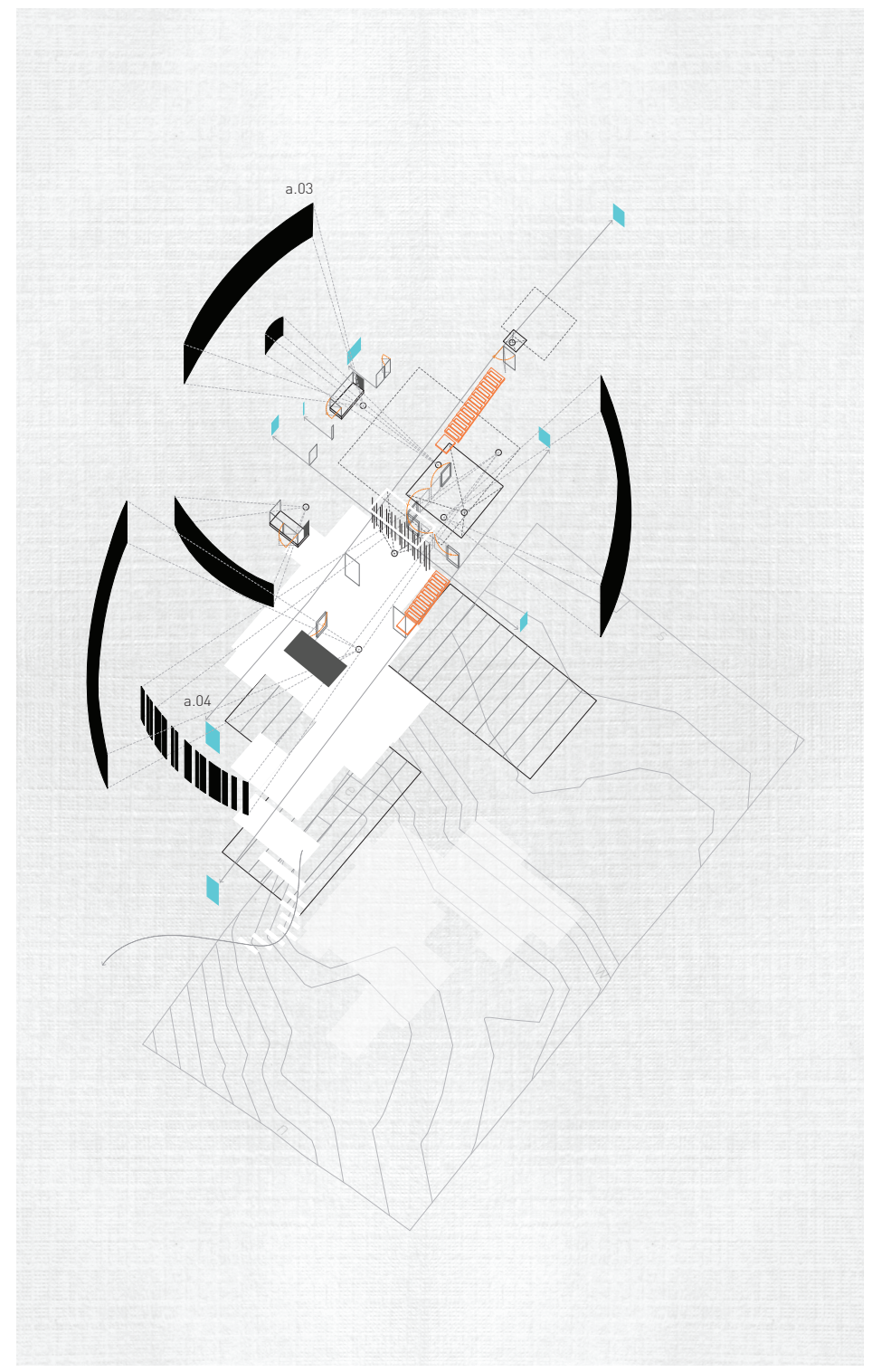

f53

Spatial and Perceptual Collision

b_Level 03 - Level 04, Sequence 02

The editing techniques of Tarkovsky and Kiarostami were used to contrast the concealing cuts for expansive reveals; the overlap of both space and time is developed through sequence shots that dissolve of and within architectural frames. Scale, proportion, and sight, play a pivotal role in creating moments of expansion within the procession. 

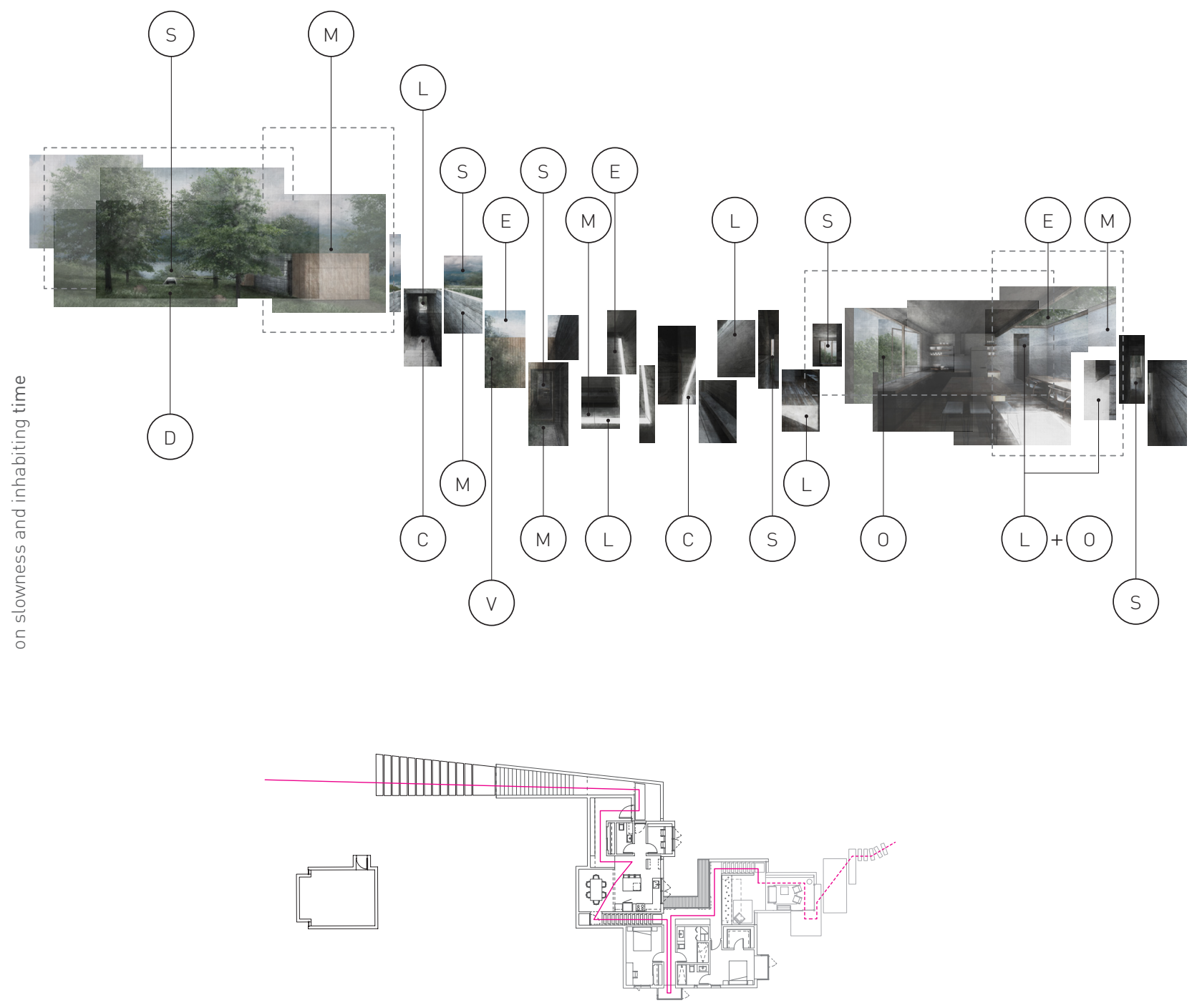

f54

Passage; Sequence 02

The experimental drawing for Sequence 02 outlines an inhabitant's procession while denoting where and how various Generators of Slowness are used. Unlike Sequence 01, the heightened influence from the assembly of film is represented in cut and expansive frames that define moments of quickening and slowing within a procession. 


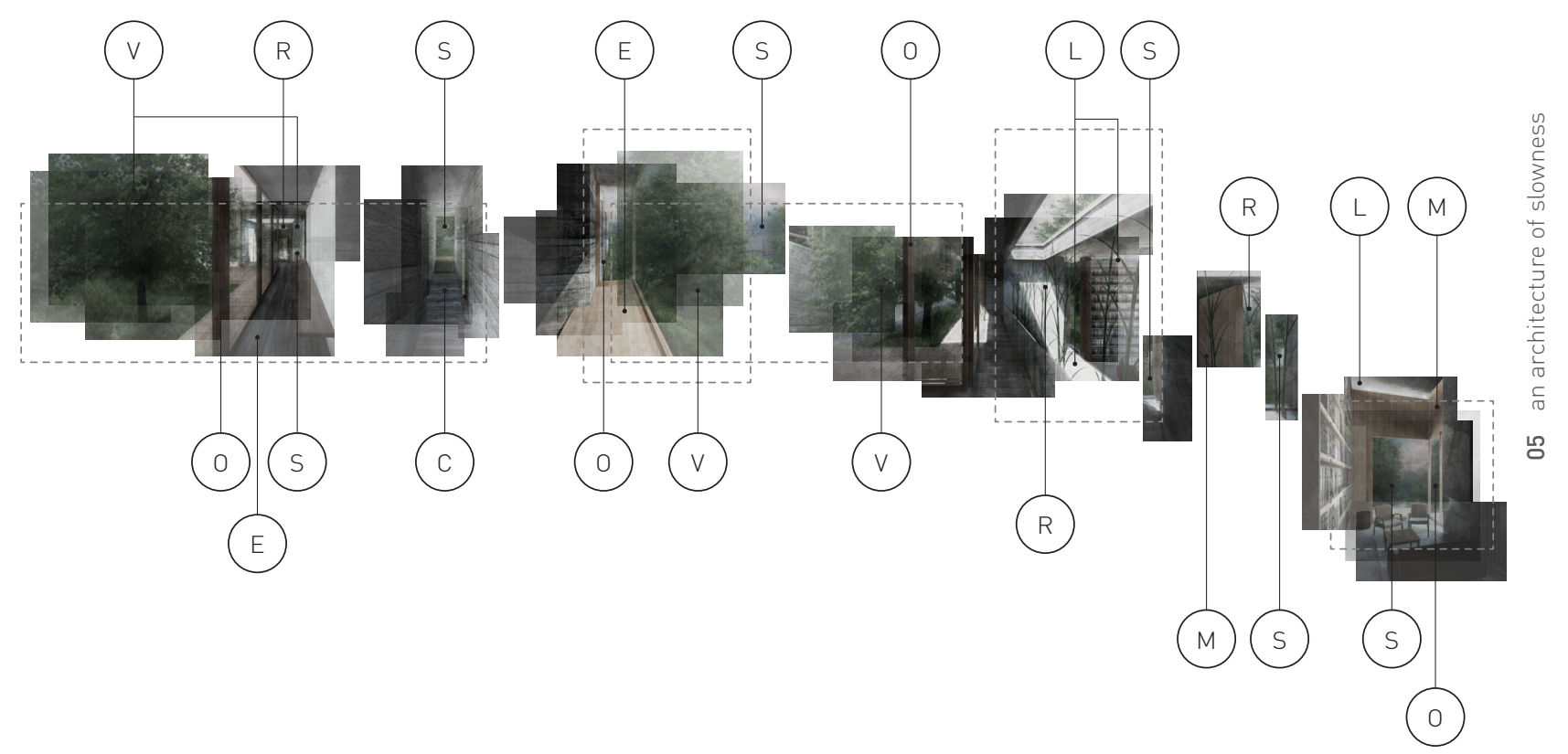

C compression E expansion R rhythm L light D darkness S sight $M$ material $\mathrm{O}$ open $\mathrm{V}$ vegetation

$$
\text { Q } 0 \text { m } 4 \quad 8
$$




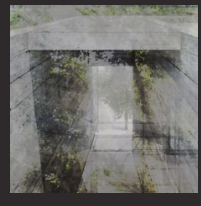

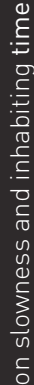

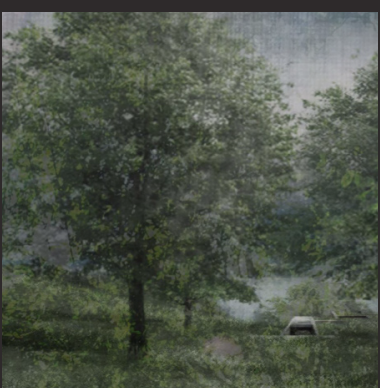

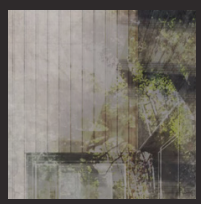
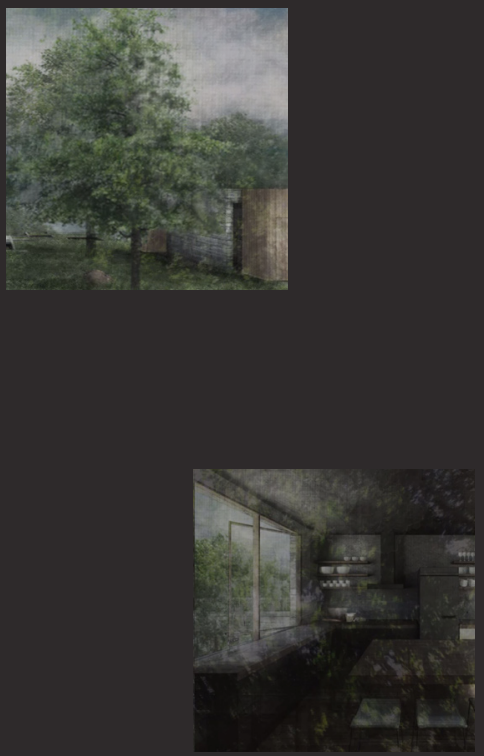

f55

Everyday Filmic Passage

Sequence 02

The film, Everyday Filmic Passage for Sequence 02 is assembled in a montage whereby cuts are used between frames to condense space and time, while sequence shots are used to counter the cut frames.

This juxtapositon between cuts and sequence shots emphasizes

the moments of frenetic speed and slowness during a continuous procession through the dwelling

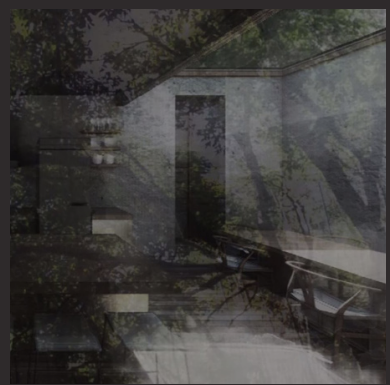




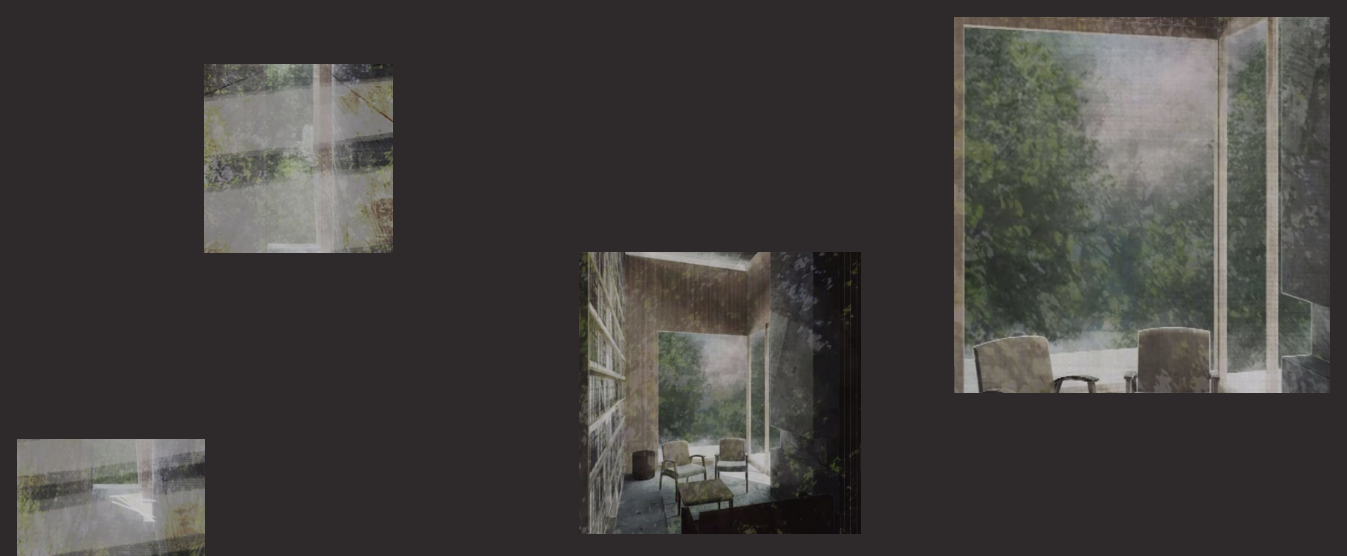

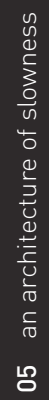

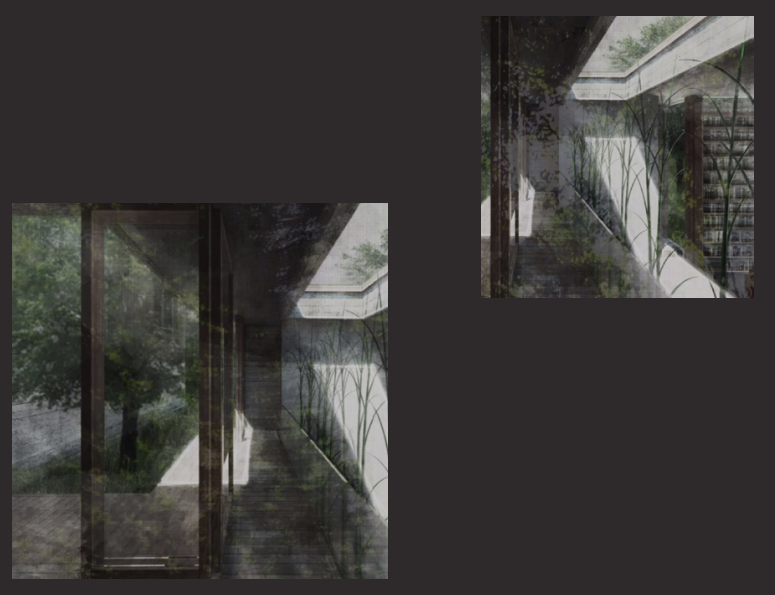




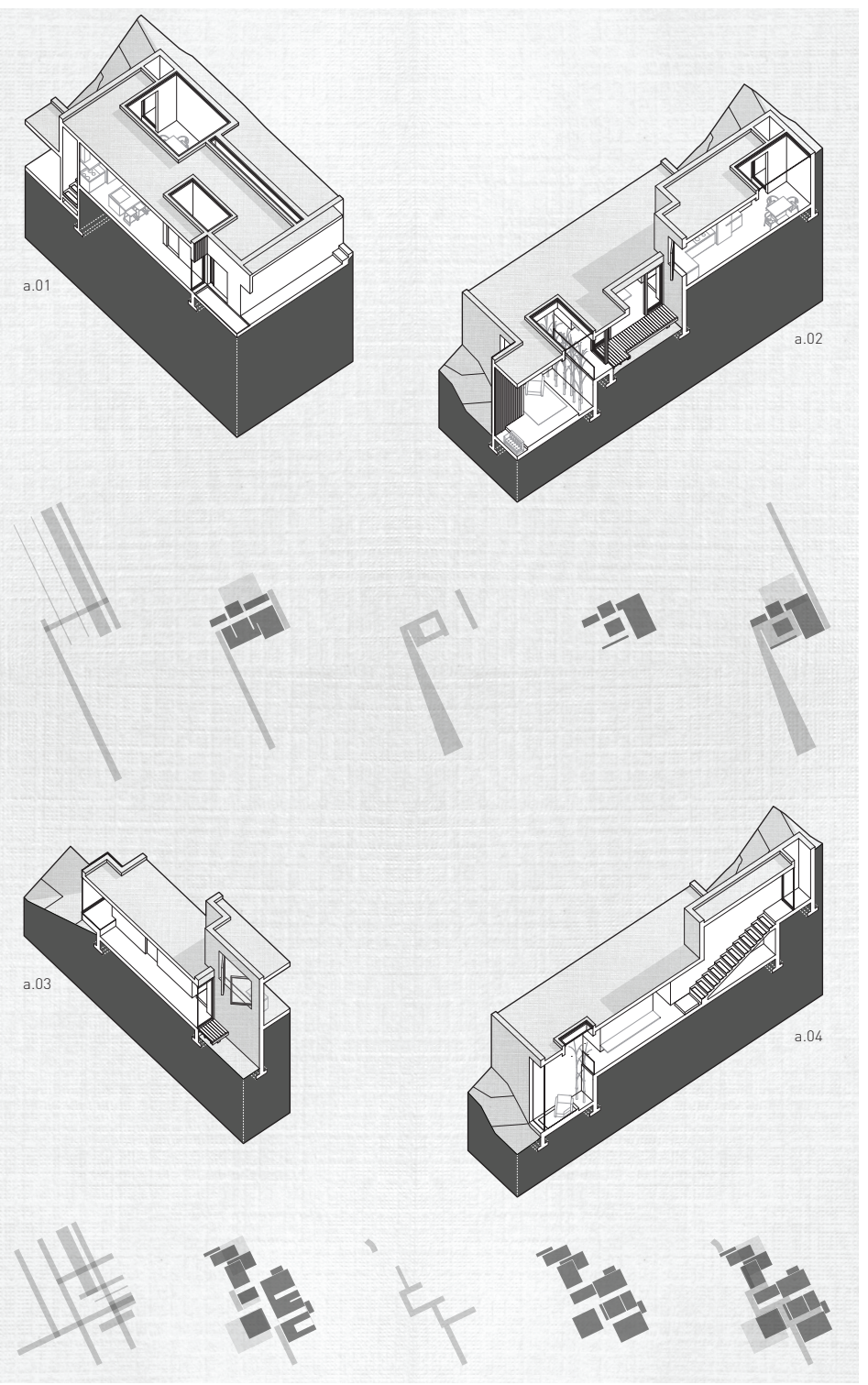

f56

Spatial Overlap and Axis Study

This study illustrates the dwelling's spatial and temporal overlapping while highlighting it's four distinct expansive axes. In order to bring the outside in and the inside out these axes allow for geological and cyclical elements of time to penetrate into the every day linear time of an inhabitant. The overlapped elements in the diagram above illustrate how spaces for stillness and motion juxtapose to create moments of quickening and slowing. 


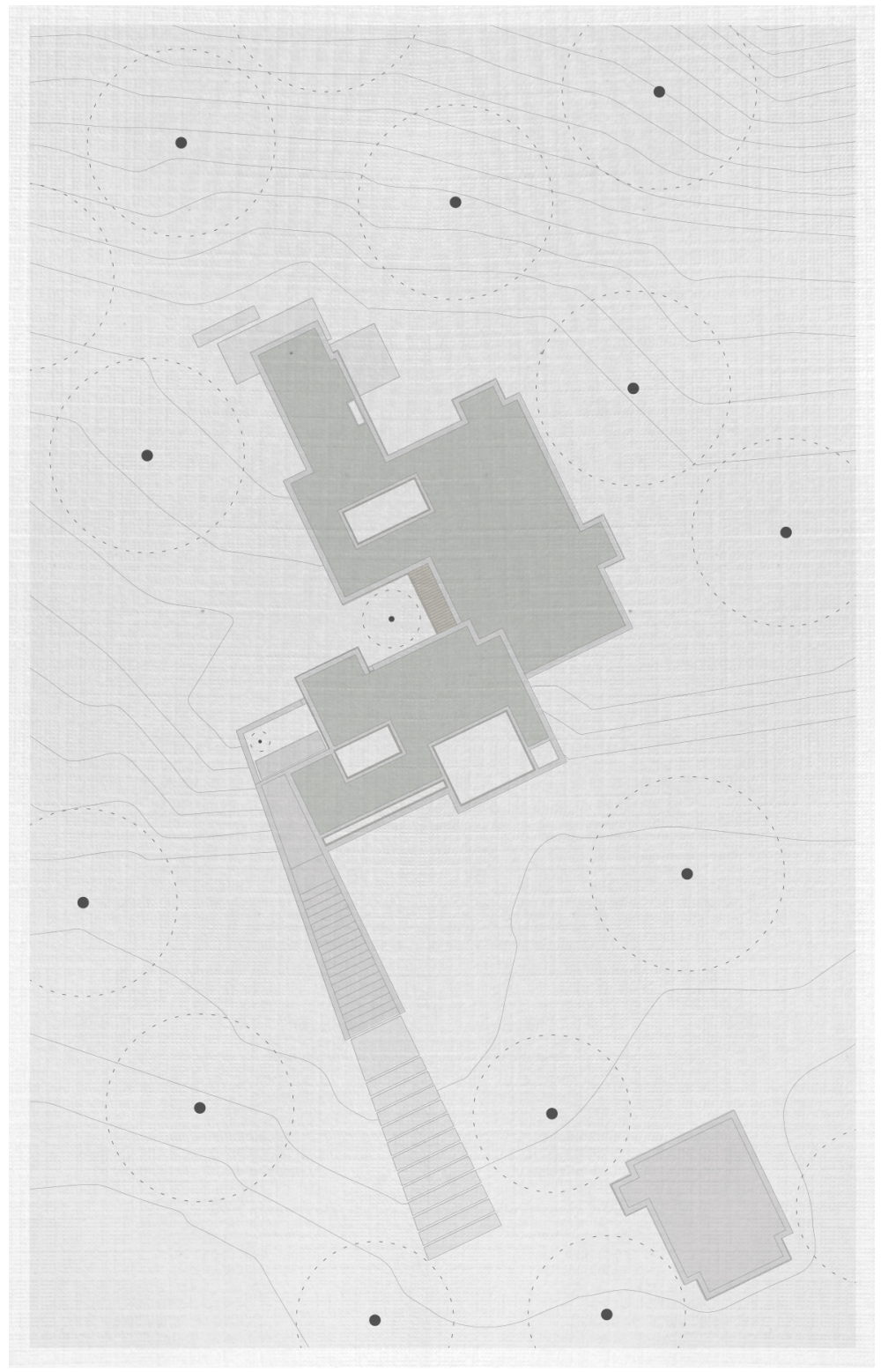

f57

Orthographic Drawings

a_Roof, Sequence 02

Situated on a heavily forested cliff overlooking the Pacific Ocean, the green roof of the sunken house aids in blending it into the landscape. The only visible feature is it's garage, which references the house, both both in form and matierality.

(1) $0 \mathrm{~m} \quad 3 \quad 6$ 

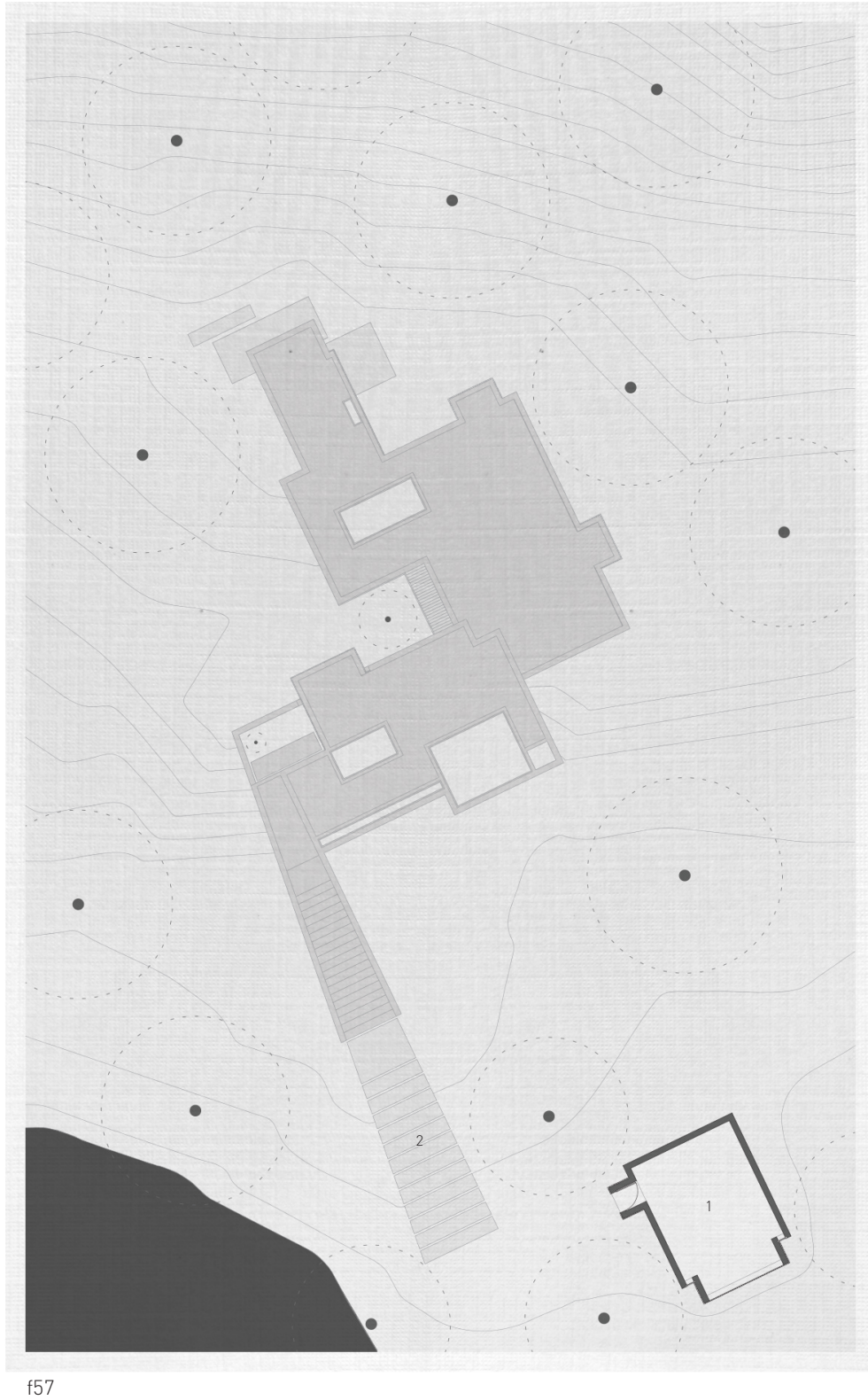

$$
\text { Orthographic Drawings }
$$

b_Level 01, Sequence 02

1 garage 2 landscape steps 


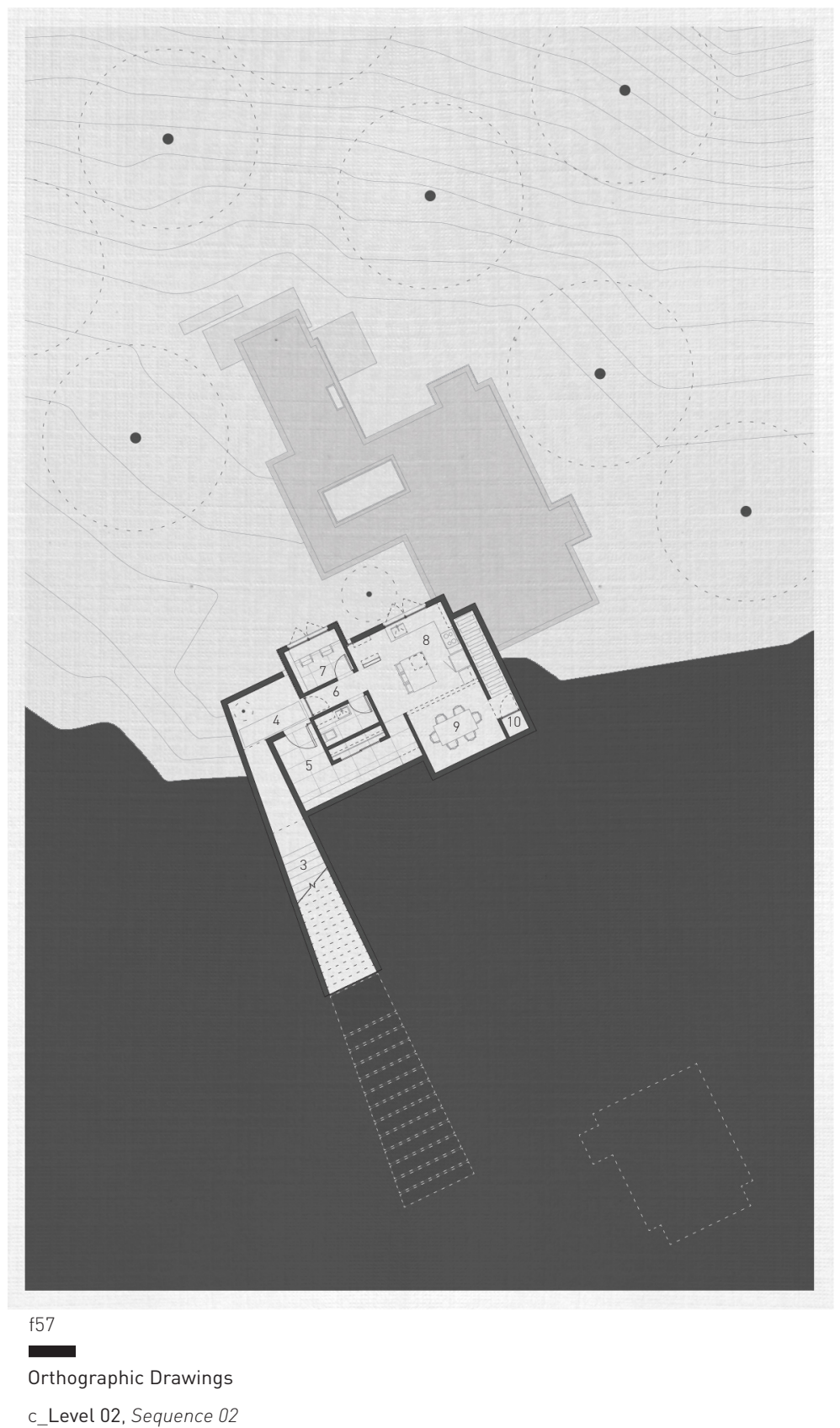

3 entrance steps 4 entrance rock garden 5 foyer 6 shortcut 7 study 8 kitchen 9 dining room 10 lightwell

(1) $0 \mathrm{~m} \quad 3 \quad 6$ 

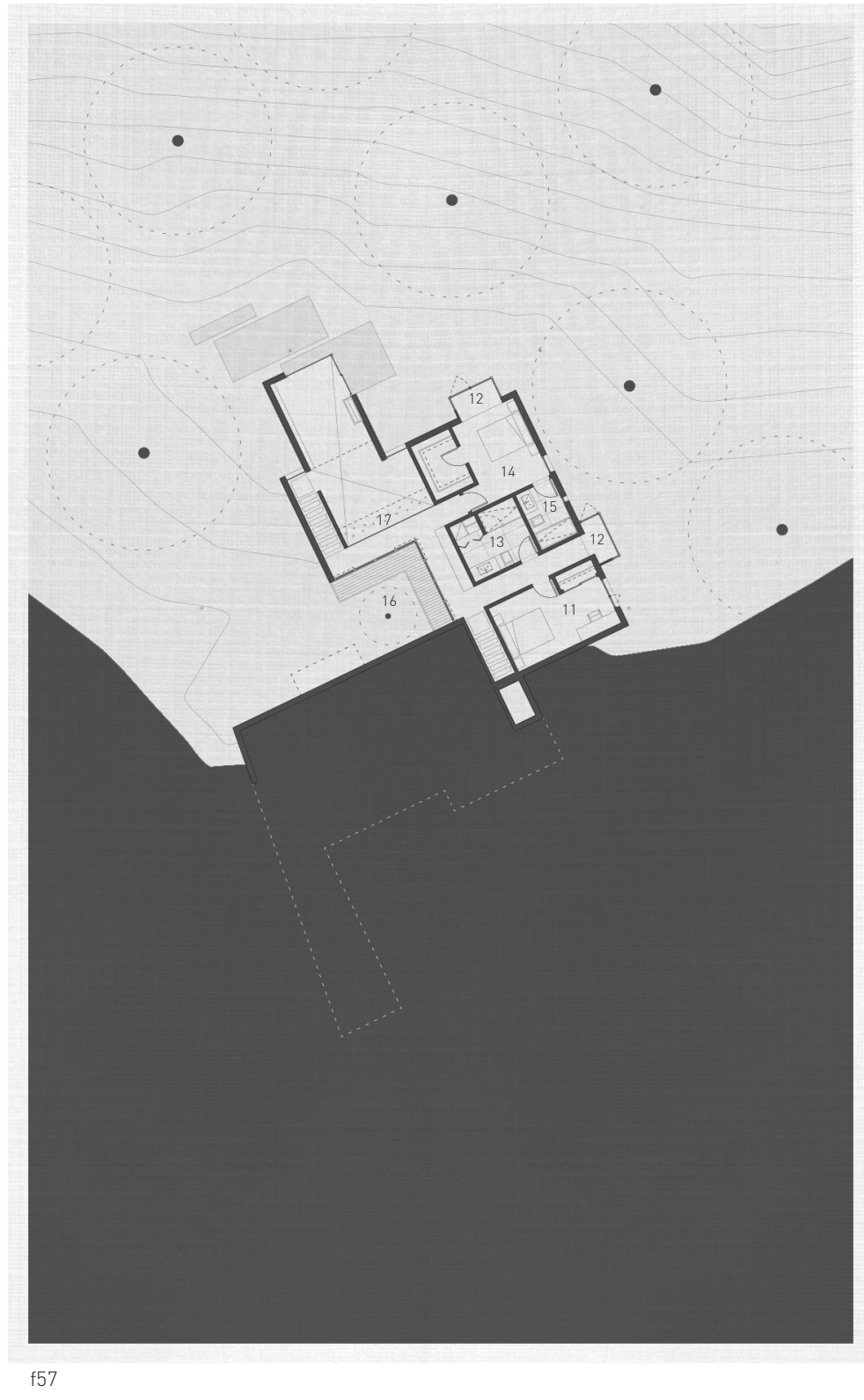

Orthographic Drawings

d_Level 03, Sequence 02

11 guest bedroom 12 bench 13 main bathroom 14 master bedroom 15 ensuite 16 green garden 17 bamboo wall 


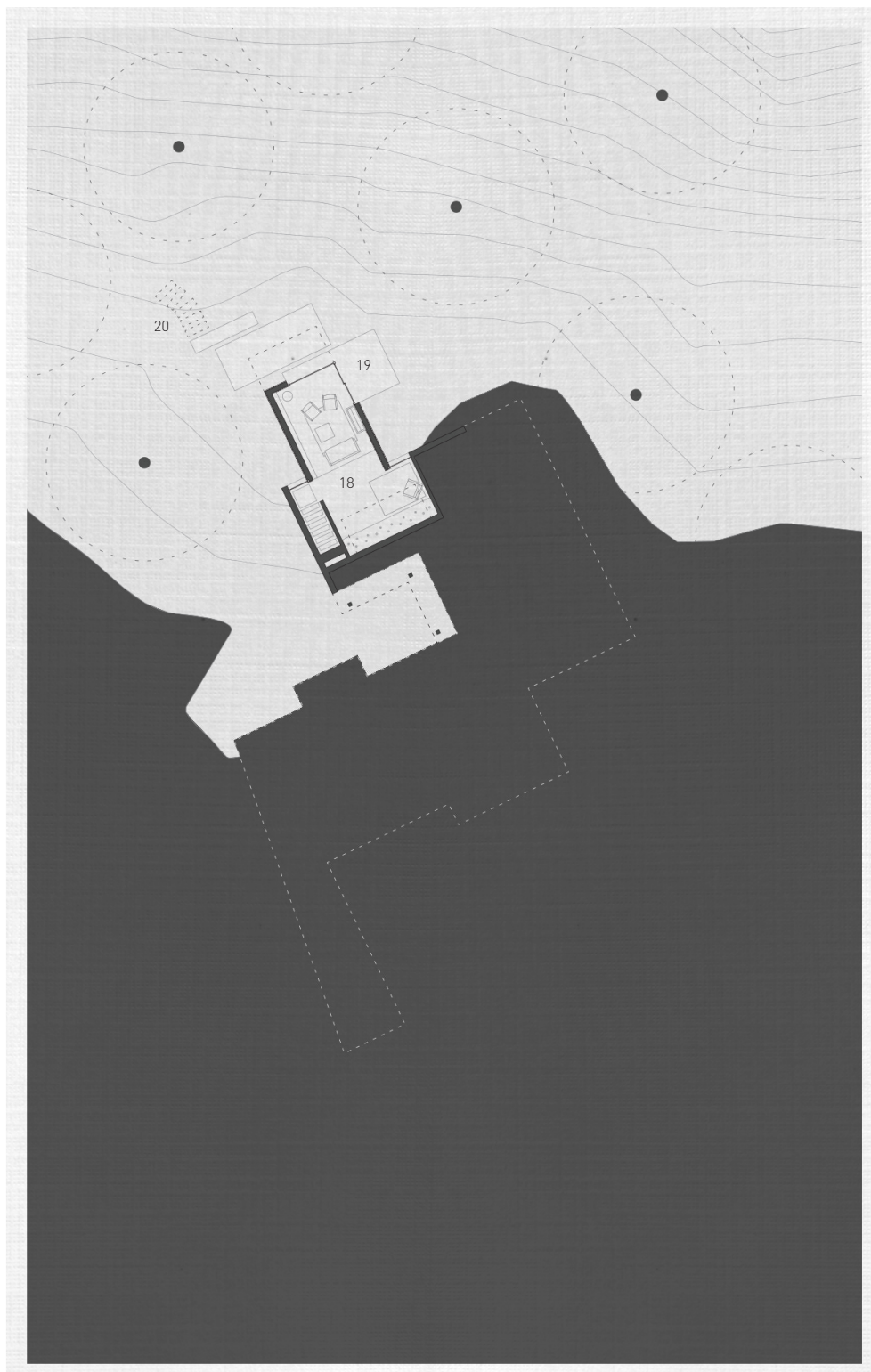

f57

Orthographic Drawings

e_Level 04, Sequence 02

18 living room 19 patio 20 stairs to water

(1) $0 \mathrm{~m} \quad 3 \quad 6$ 


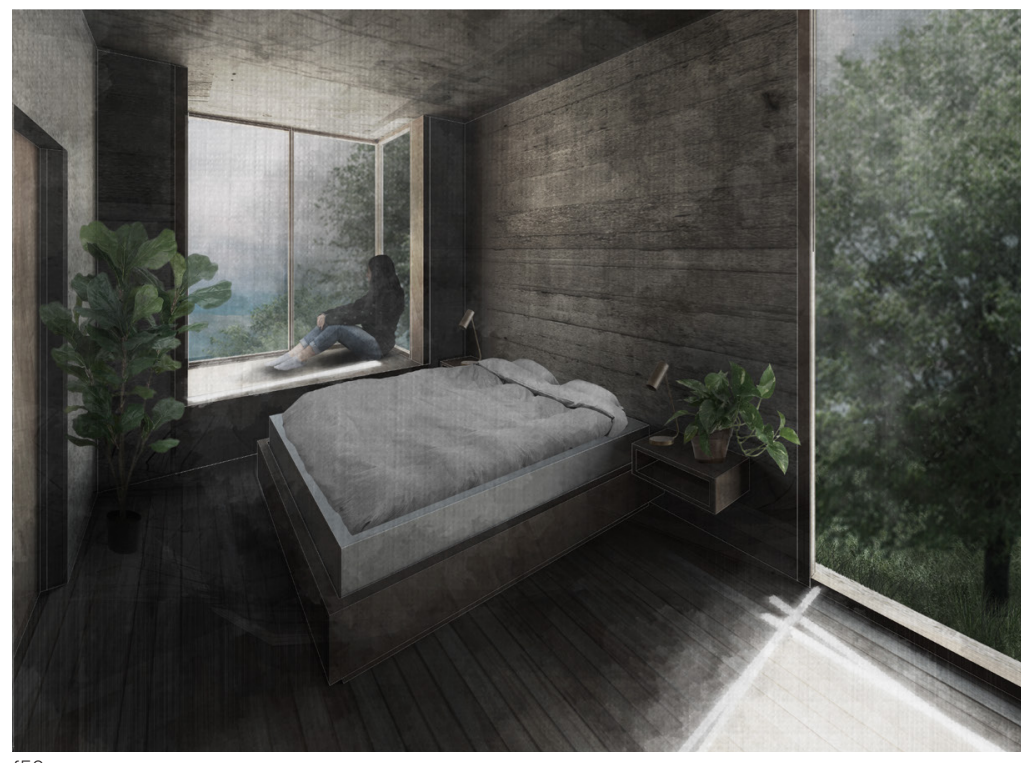

f58

Everyday Inhabitance

a_Wake Up, Sequence $02 \quad 0$

\begin{tabular}{|c|c|c|c|c|c|c|}
\hline$\bullet$ & 0 & & 0 & 0 & 0 & 0 \\
\hline 01 & 02 & 03 & 04 & 05 & 06 & 07 \\
\hline 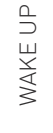 & 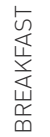 & $\begin{array}{l}\frac{r}{c} \\
\text { Or } \\
3\end{array}$ & $\begin{array}{l}z \\
\text { 㟧 } \\
\text { 亲 } \\
0\end{array}$ & $\begin{array}{l}\text { 妥 } \\
\text { 玄 }\end{array}$ & 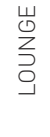 & $\begin{array}{l}\text { 岃 } \\
\text { ज }\end{array}$ \\
\hline
\end{tabular}


As the sun begins to rise, the bedroom fills with light; it bounces off the walls and floor awaking one while the other continues sleeping. Shortly after the sound of the water crashing against the rocks fills the space and awakes the other. The smell of coffee from the kitchen mixes with the wet grass from the night's rain and the Pacific Ocean. The woman decides to take a moment in her routine to sit and watch the day start. Perching up on the wooden bench, she admires the water, cars, and people as they begin another day, just as she is about to.

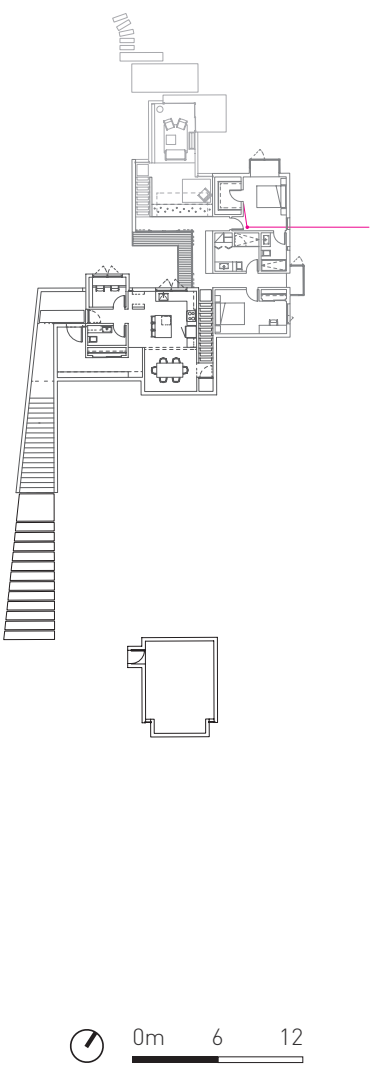




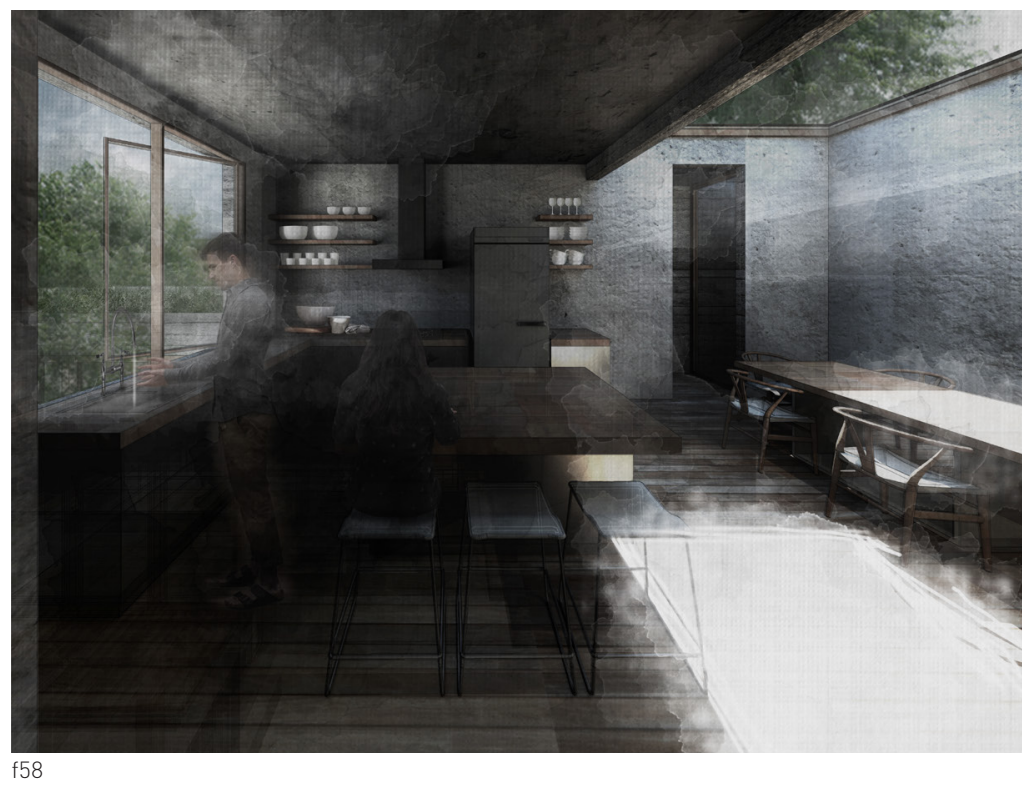

Everyday Inhabitance

b_Breakfast, Sequence 02 O

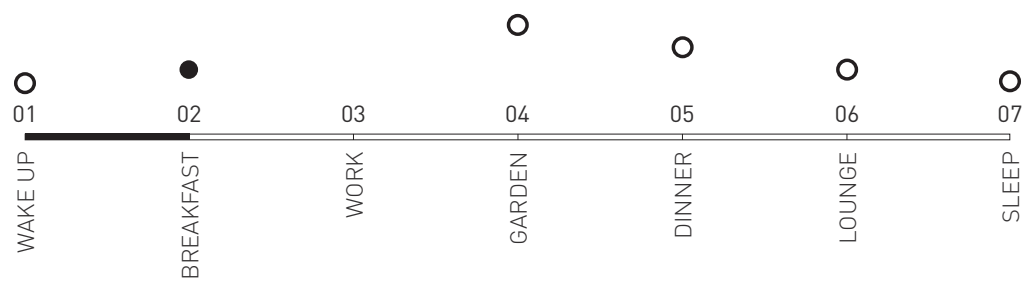


She ascends a narrow set of stairs and notices the lightwell is wet from the rain. As she enters the kitchen, she looks up through the skylight and out the open casement window to see the trees flowing in the wind and makes note of the clear and crisp day. She loves the coast after it rains, the smell lingers in the air and wetness on the ground stays for quite some time. Her partner has already begun breakfast, and she decides to assist him but can't help being distracted by the beautiful day outside. Once breakfast is ready they decide to sit by the window and take in the day.

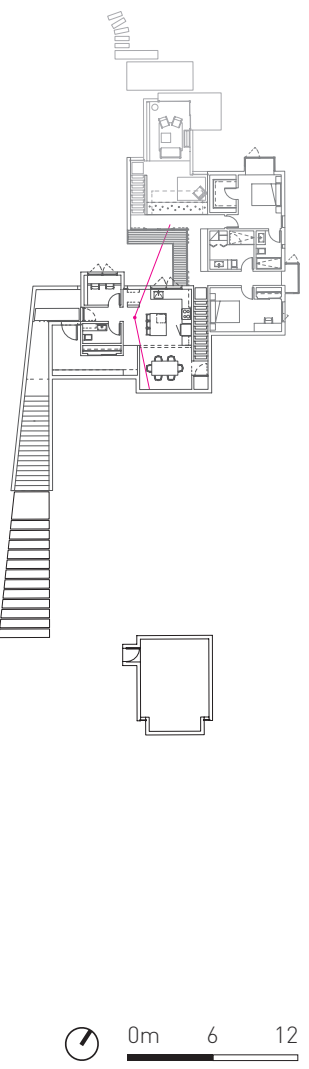




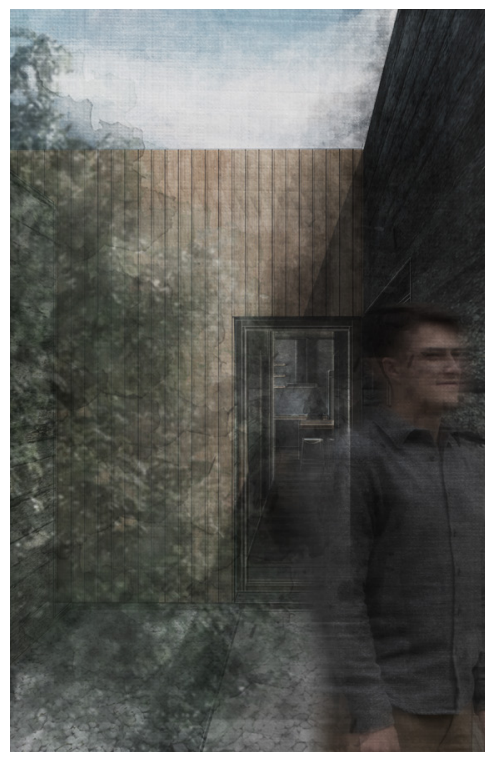

f58

Everyday Inhabitance

c_Work, Sequence 02

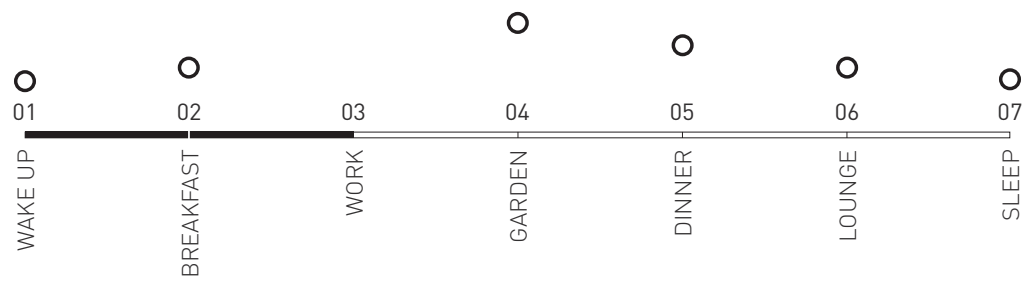


After breakfast, her partner descends to get ready for work; She cleans up and joins him. They close the garden doors and notice the deck is still wet from the rain. Ascending towards the kitchen, they grab their bags and move through a narrow corridor to the closet for an umbrella. A single slit skylight illuminates the space as they sit on the concrete bench and change into their work shoes. She runs into the kitchen to grab another snack while her partner climbs the stairs to the garage. Afterwards, she proceeds through the dimly lit foyer and out into the rock garden; ascending the expanded staircase makes her physically aware of her exhaustion and the number of steps she traverses daily. She enters the car and continues to work.

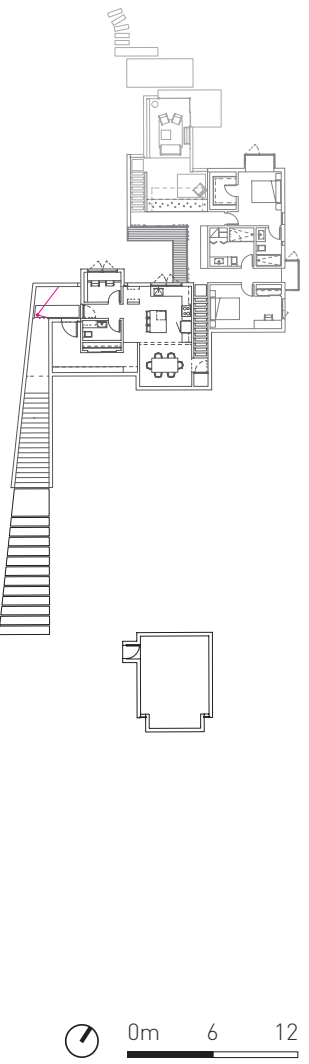




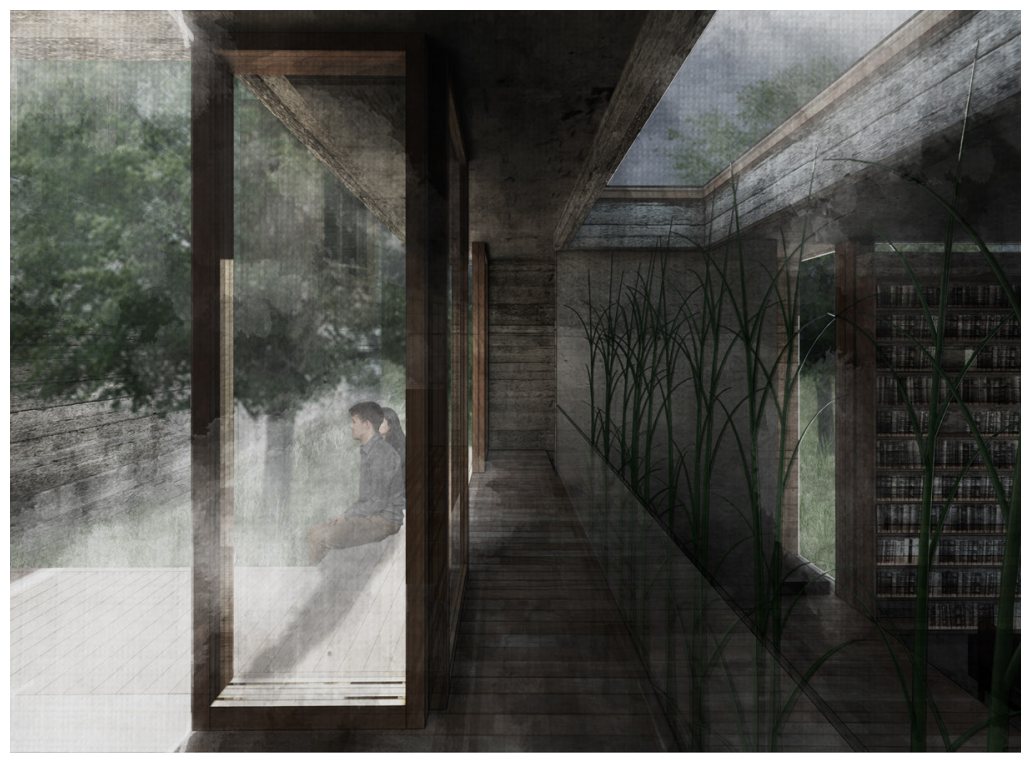

f58

Everyday Inhabitance

d_Garden, Sequence 02 O

\begin{tabular}{|c|c|c|c|c|c|c|}
\hline 0 & 0 & & & 0 & 0 & 0 \\
\hline 01 & 02 & 03 & 04 & 05 & 06 & 07 \\
\hline 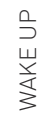 & 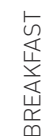 & 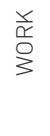 & $\begin{array}{l}z \\
\text { 崇 } \\
\text { 委 }\end{array}$ & $\begin{array}{l}\text { 妥 } \\
\frac{\text { Z }}{0}\end{array}$ & 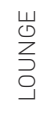 & $\begin{array}{l}\text { 岀 } \\
\text { ज }\end{array}$ \\
\hline
\end{tabular}


After a long day of work the couple decides to de-stress in the garden. They open the glass doors allowing the elements, the wind and light, to enter the interior of the house. The couple sit on the wooden deck and listen to it creaking as they swing their feet gently and graze the grass beneath them. The woman steps down off the deck and onto the ground and notices it is still wet from the rain, so she gets back on the deck and allows her feet to dry. They sit for some time, allowing the sun to set while they discuss their day and what to do that evening.

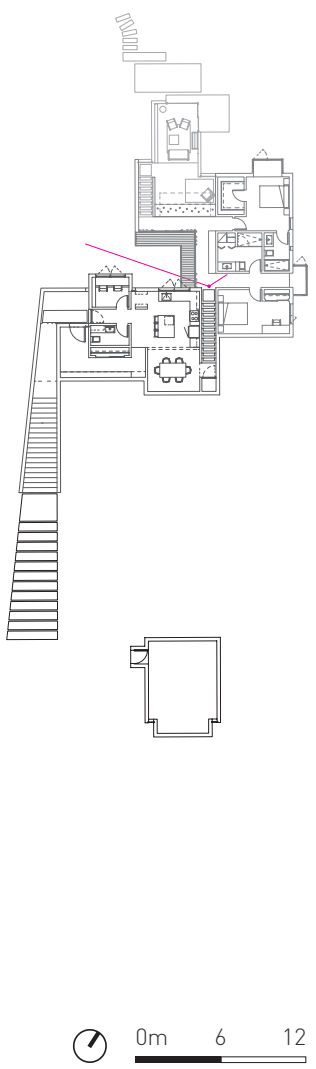




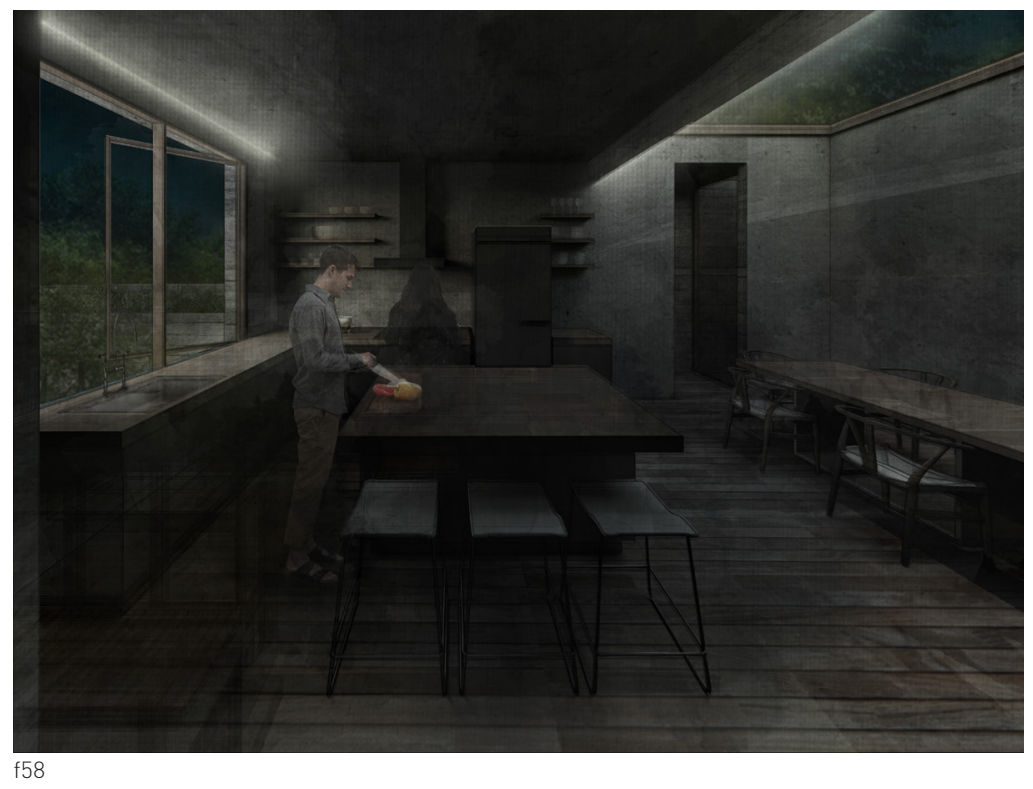

Everyday Inhabitance

e_Dinner, Sequence $02 \quad 0$

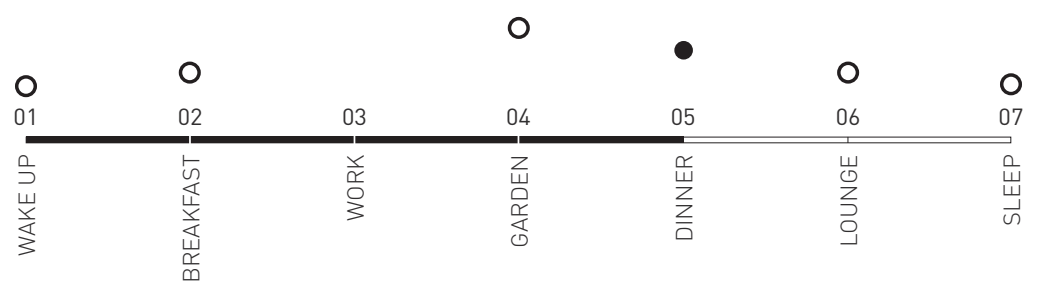


After watching the sunset the couple ascends to the kitchen and notices the deep blue of the evening sky through the skylight and the open casement window. As they cook they hear crickets creaking while the ocean crashes against the rocky terrain. They play Bossa Nova at a low volume so as to meld the music with the atmosphere of the coast. While the food cooks in the oven the kitchen fills with the smell of roast vegetables and breaded chicken. Finally, they plate and sit at the dining room table to enjoy their meal under the skylight beneath the stars.

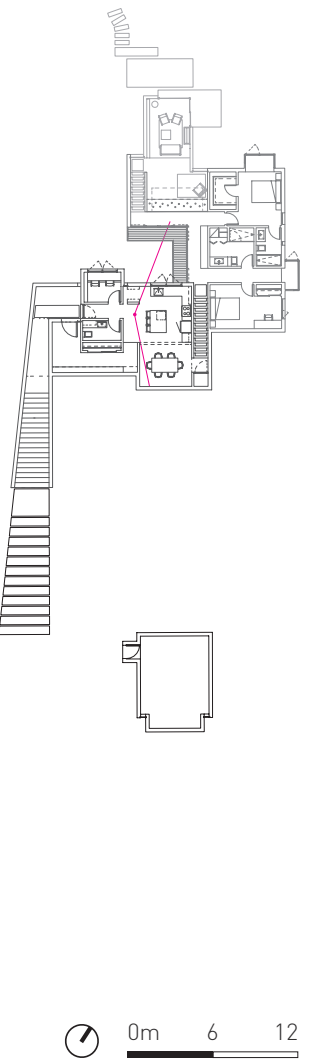




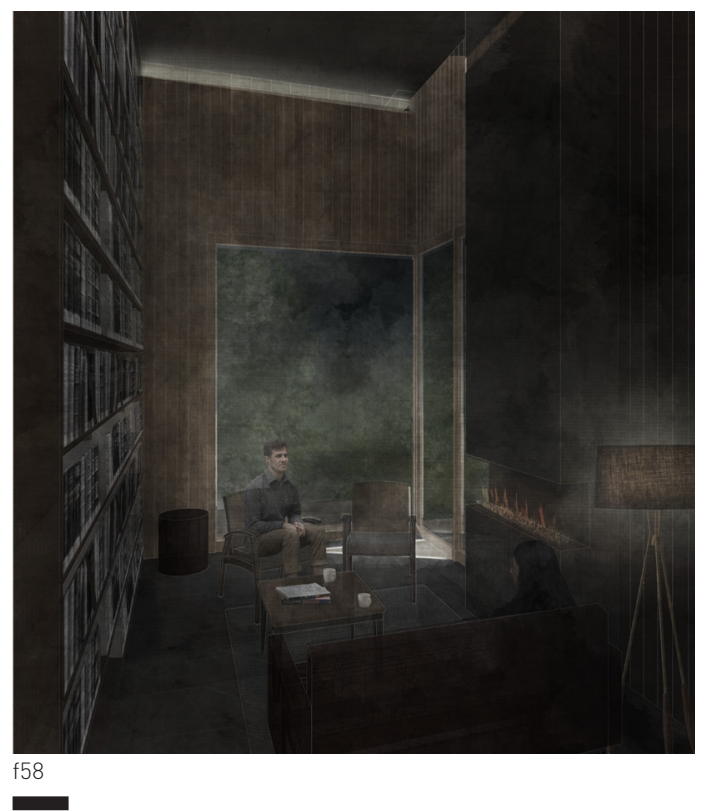

Everyday Inhabitance

f_Lounge, Sequence $02 \quad 0$

\begin{tabular}{|c|c|c|c|c|c|c|}
\hline 0 & O & & 0 & 0 & • & 0 \\
\hline 01 & 02 & 03 & 04 & 05 & 06 & 07 \\
\hline $\begin{array}{l}\text { a } \\
\text { 山े } \\
\frac{3}{3}\end{array}$ & 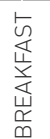 & $\begin{array}{l}\frac{r}{c} \\
\text { Or } \\
3\end{array}$ & $\begin{array}{l}z \\
\text { 㟧 } \\
\text { 亲 } \\
0\end{array}$ & $\begin{array}{l}\text { 妥 } \\
\text { 玄 }\end{array}$ & 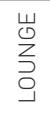 & $\begin{array}{l}\text { 岀 } \\
\text { 山 }\end{array}$ \\
\hline
\end{tabular}


The couple cleans up the kitchen and makes tea to take to the living space. They descend twice, once towards the garden and another towards the living space. Here, they sit with a cup of tea and read as the night sky slowly shifts from blue to black. The nights are quite chilly on the coast and the fire place brings warmth into the space. After some time the woman steps up to the clear space in front of the bamboo wall and lays out a yoga mat. She practices and returns to the couch more relaxed than before. As the night winds down, they begin to feel tired and decide to retreat to the bedroom.

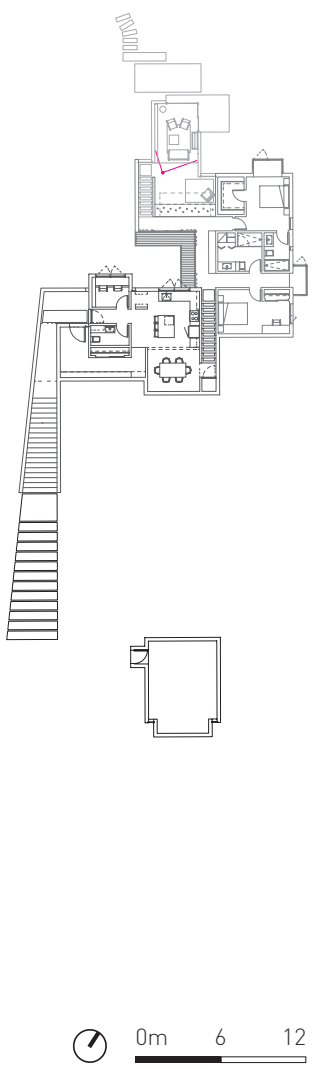




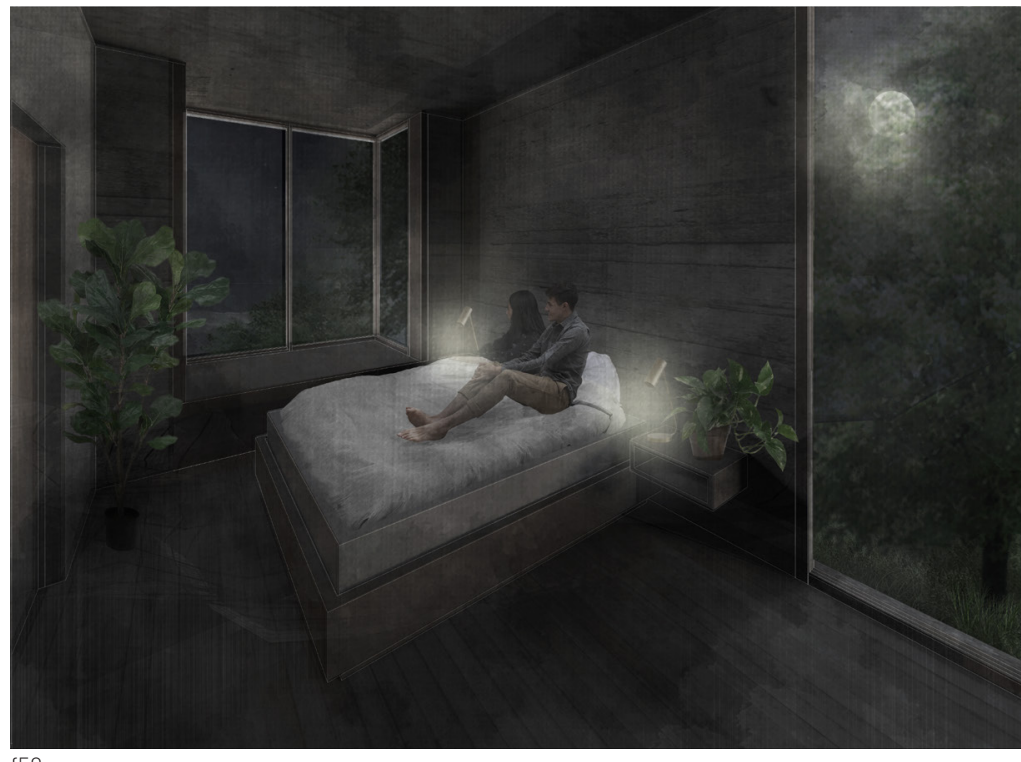

f58

Everyday Inhabitance

g_Sleep, Sequence 02

O

\begin{tabular}{|c|c|c|c|c|c|c|}
\hline 0 & 0 & & 0 & 0 & 0 & • \\
\hline 01 & 02 & 03 & 04 & 05 & 06 & 07 \\
\hline $\begin{array}{l}\stackrel{0}{S} \\
\overleftrightarrow{山} \\
\stackrel{y}{3} \\
3\end{array}$ & 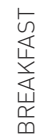 & $\begin{array}{l}\frac{y}{\alpha} \\
\stackrel{0}{0} \\
\stackrel{3}{3}\end{array}$ & 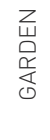 & 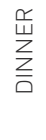 & 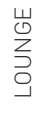 & $\begin{array}{l}\text { 岀 } \\
\text { ज }\end{array}$ \\
\hline
\end{tabular}


At night, darkness overtakes the atmosphere of the coast while the sounds of the water, the moon's light shining onto the landscape, and the smell of the crisp ocean air heightens the atmosphere for the inhabitants. A blankness overcomes the couple and the bedroom in order to allow for stillness to take over. The couple lay in bed for quite some time discussing the following day until their tiredness consumes them. The bedside lamps are turned off and darkness washes over the space as the single illuminating element becomes the moon. The hectic modern world is lessened with moments of pause that bring the inside out and the outside in, connecting geological and cyclical time with the linear time of an inhabitant.

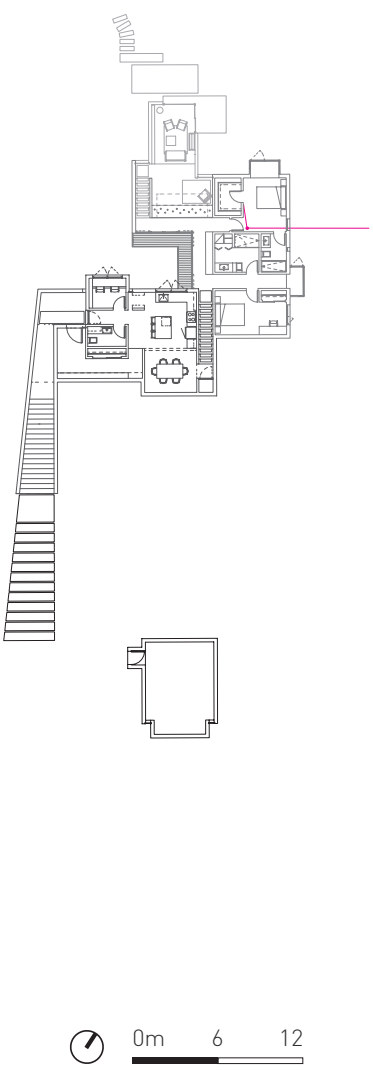




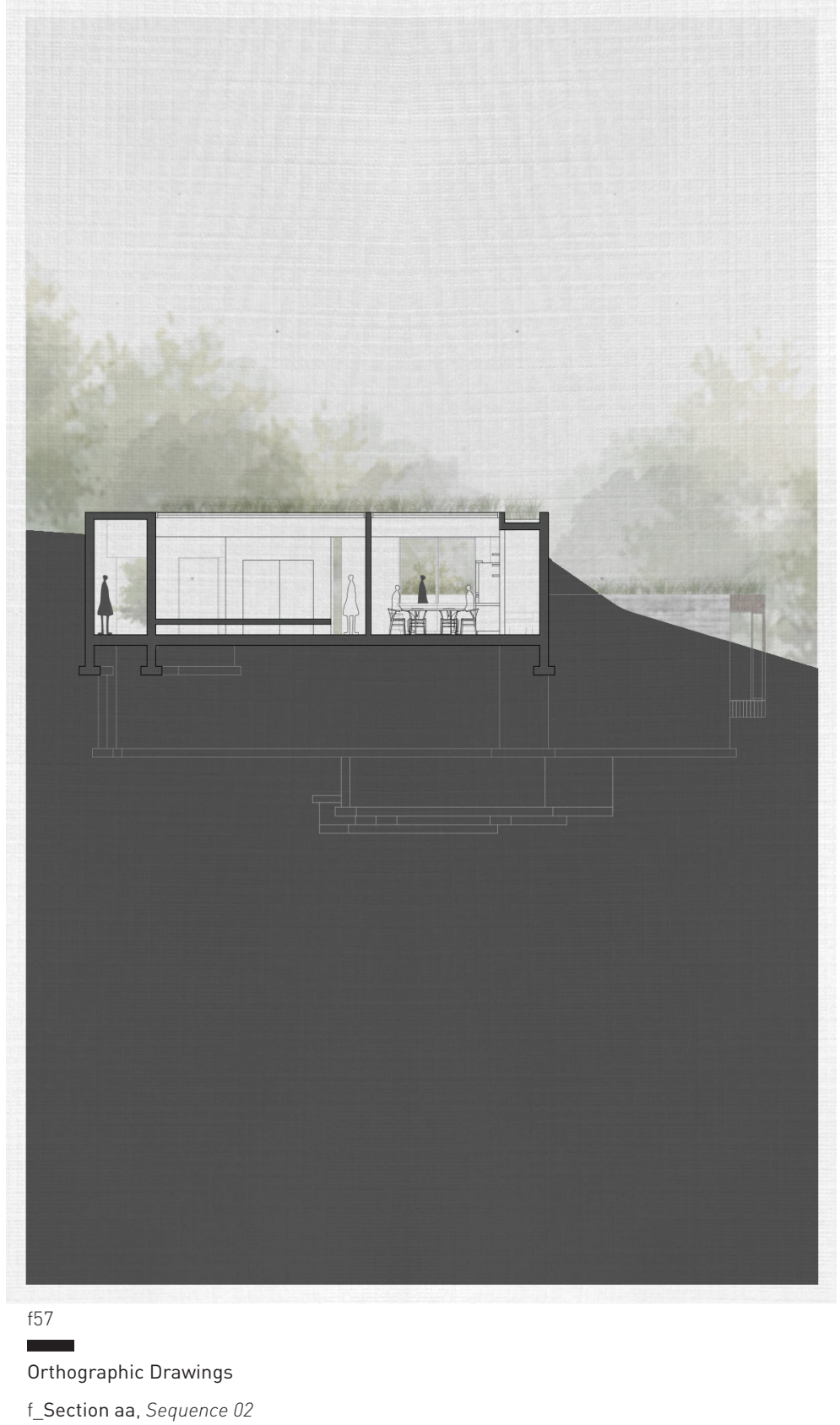




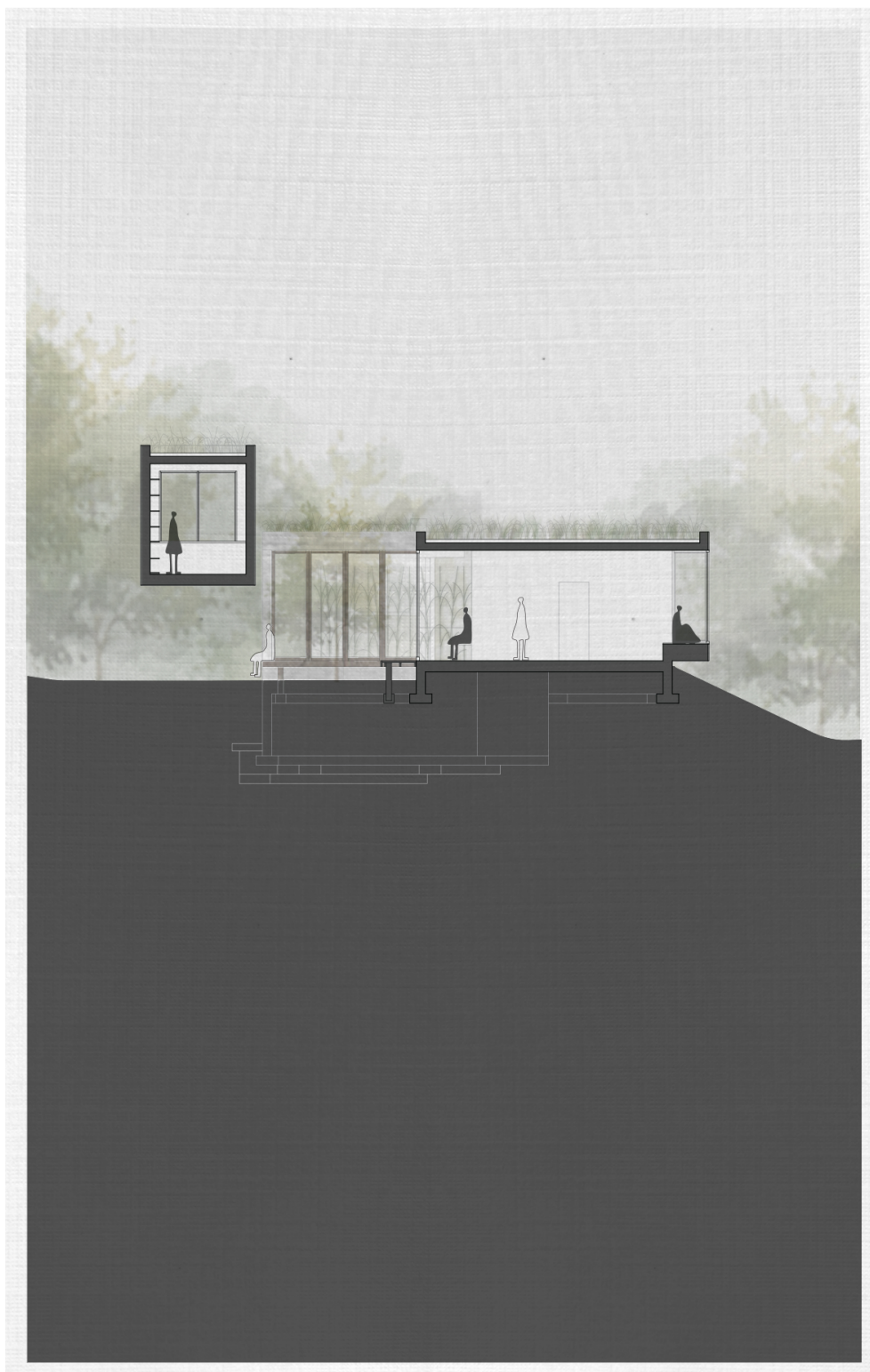

f57

Orthographic Drawings

g_Section bb, Sequence 02

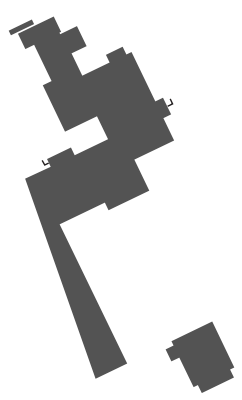

(1) $0 \mathrm{~m} \quad 3 \quad 6$ 


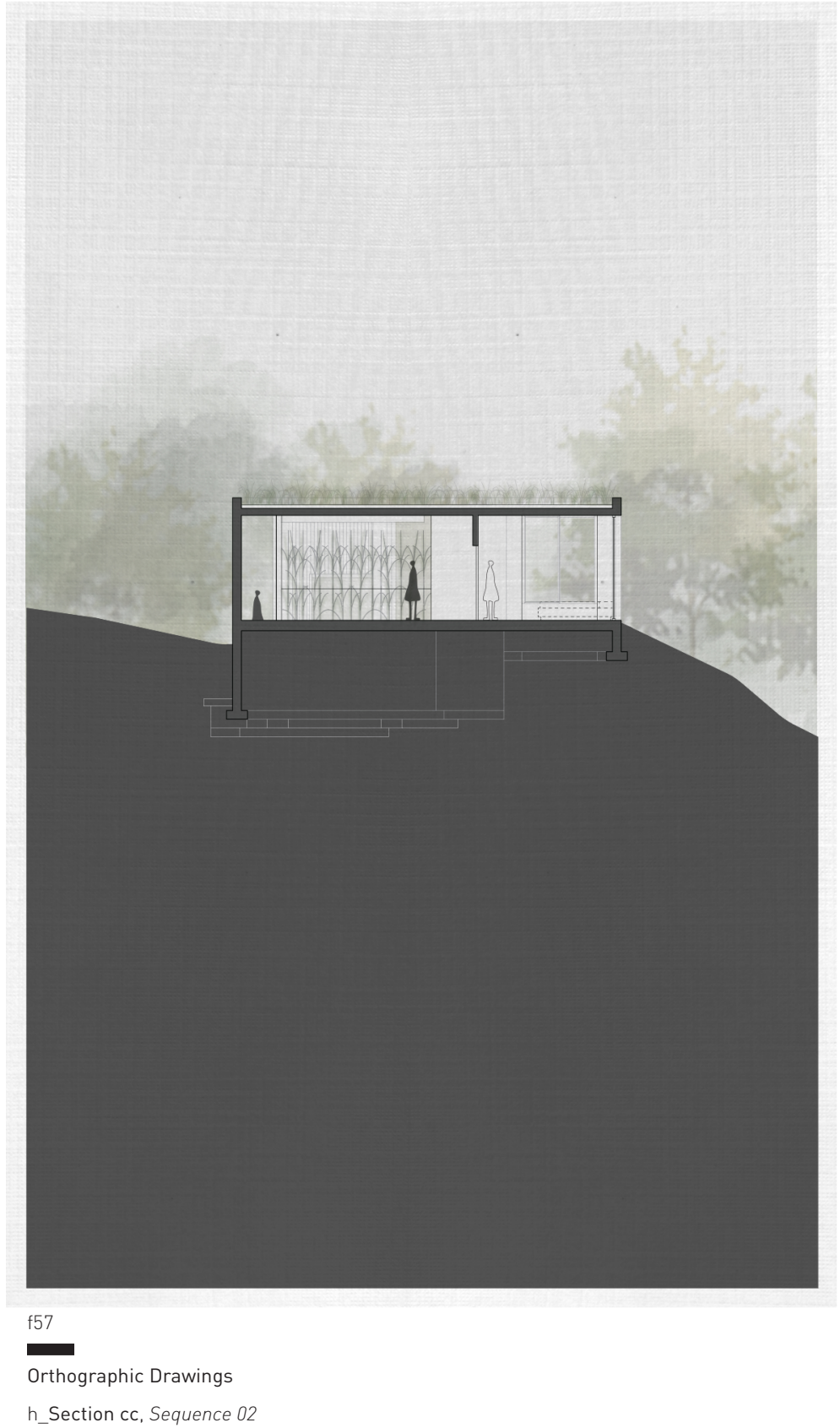




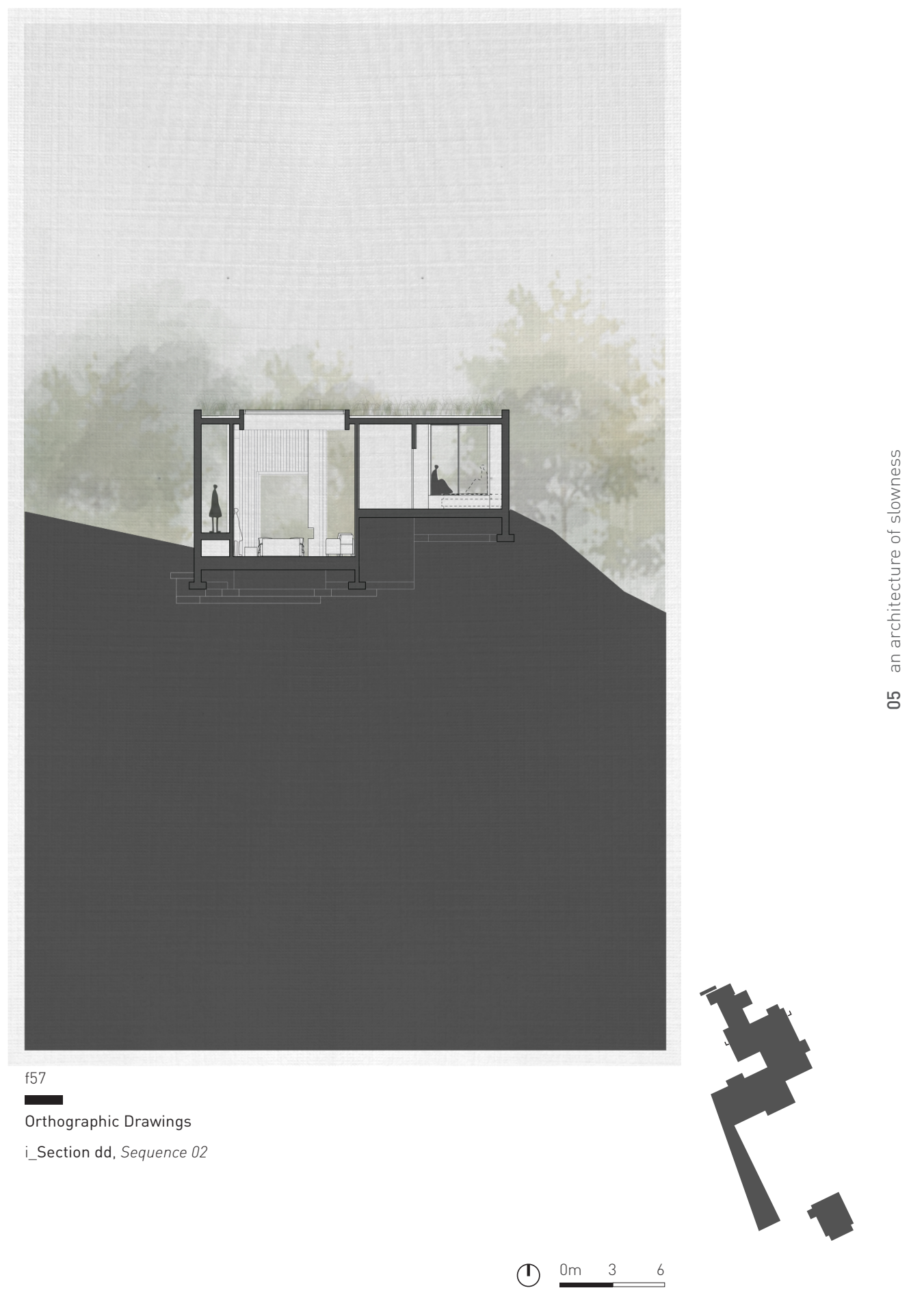




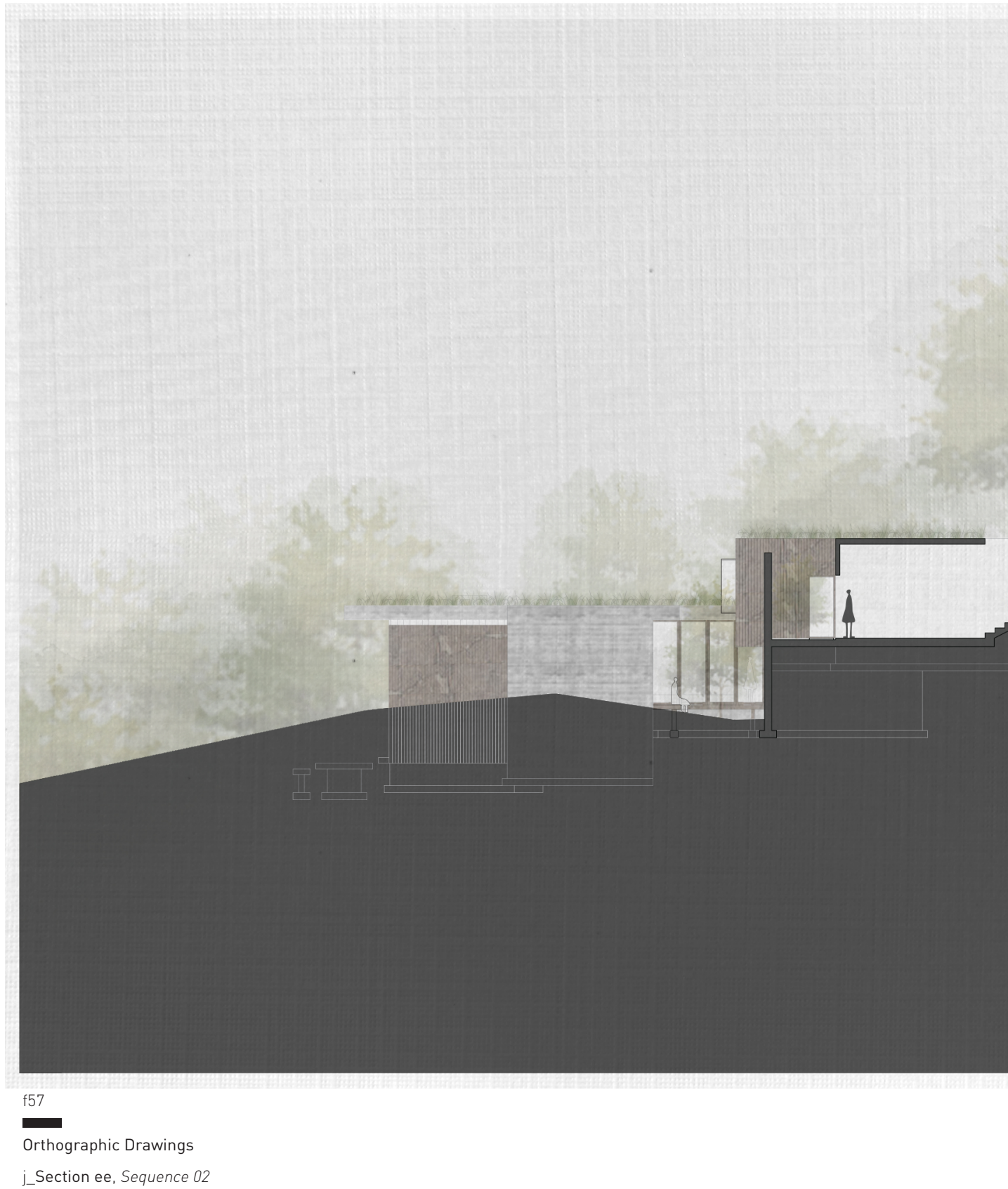


(1) $0 \mathrm{~m} 3 \quad 6$ 


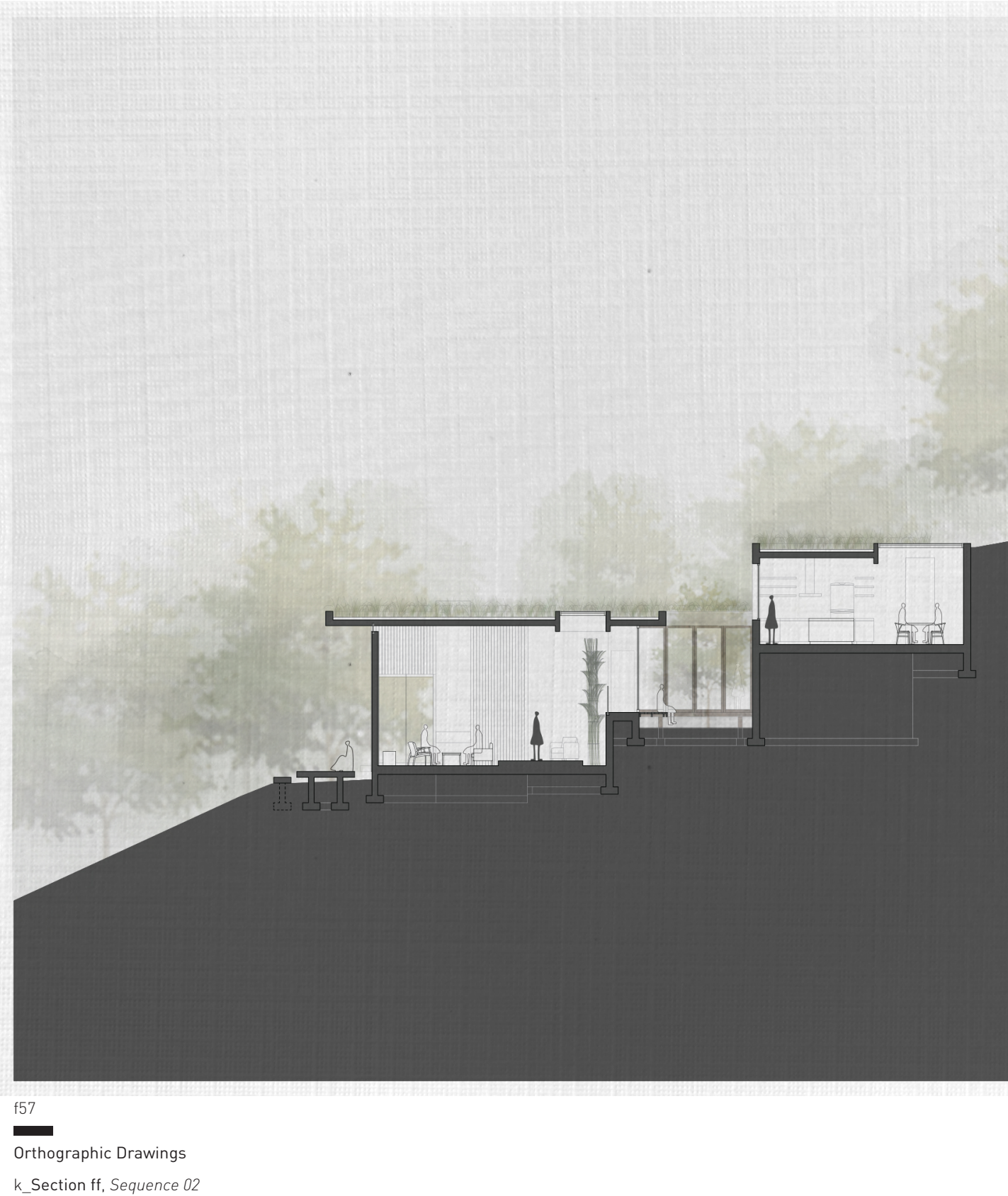


(1) $0 \mathrm{~m} 3 \quad 6$ 


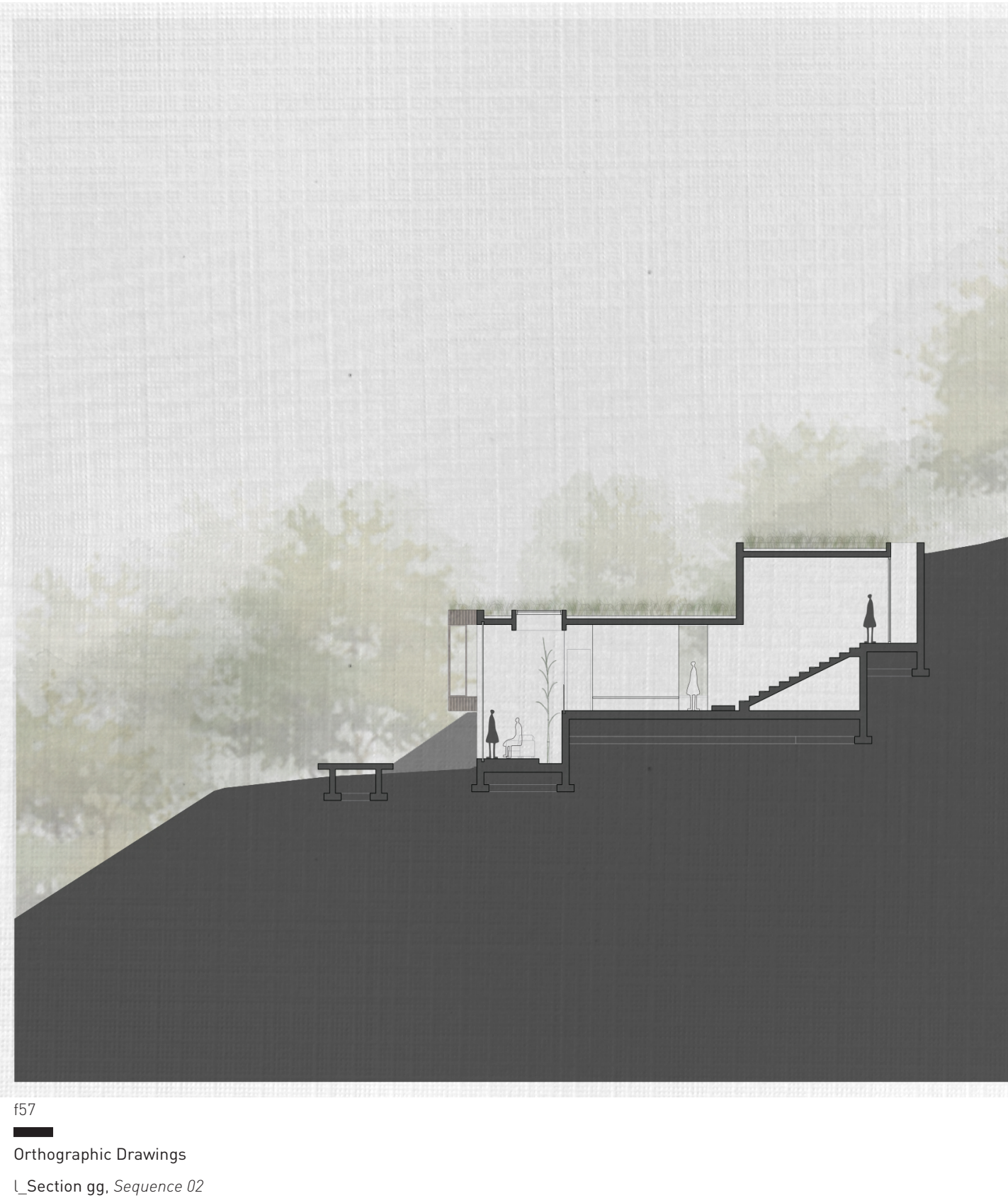


(1) $0 \mathrm{~m} 3 \quad 6$ 
By shifting focus from clock time to experiential time, slowness acts as a tool to combat the contemporary fascination with frenetic speed and its resultant distortion of the everyday. Through spatial and perceptual connectivity, this thesis explored the perception and reception of architectural frames or spaces.

An architecture of slowness resides in the interplay between three distinct architectural mediums: duration, presence, and place. With duration, the sequential nature of architecture is represented; research into filmic frames discovered the implications of speed and density both of and within architectural frames and the resultant experience of space and time. With presence, the conscious experience of architecture is represented; research into the effects of speed on the mind and body identified slowing down as the activity that produces a dialogue between subject and object or perceiver and perceived. With place, the atmospheric qualities of space are represented; research into various atmospheric generators, which have been defined as Generators of Slowness, developed a heightened emotive reading of architectural space that embeds itself into an individual's mind and body. 
Overall, an architecture of slowness is defined as an architecture that uses the elements described within duration and place to presence the mind and body, in doing so, heightens an individual's connectivity with both space and time; for Pallasmaa, "architecture articulates our experiences of time as much as of space, though we are not often conscious of it." With an architecture of slowness, the intent is to make an individual conscious of the passage of time.

\section{Notes}

1 Holl, Pallasmaa and Gómez, Questions of Perception: Phenomenology of Architecture, 30.

2 Providência, Architectonica Percepta: Texts and Images 1989-2015, 70.

3 Pallasmaa. "Inhabiting Time." Architectural Design, 54. 
appendices 
The following appendix will be presented through my personal experiences in Japan; significant buildings, temples, and gardens are used to further define the Generators of Slowness. Through firsthand experiences of the following projects, slowness and temporal inhabitance transformed from an idea to an array of concrete memories that translate themselves into an architecture of slowness.

\section{Shiba Ryōtarō Memorial Museum}

The Shiba Ryōtarō Memorial Museum by architect, Tadao Ando, was built to house and commemorate the literary figure's popular historical novels and travel writings, which provided moral support for the Japanese after World War II.

The museum is situated, with a gated entrance, in a residential area in Eastern Osaka. After entering a small garden one immediately pays for a ticket by the kiosk and continues a plant-filled trail towards the entrance of the museum. The entrance, a curved glass promenade hidden by large trees, slowly begins to reveal itself as one makes their way through the garden. During the procession, the rhythm of the mind and body heightens as one becomes increasingly mindful of the experience. Rhythm allows one to detach from the everyday and enter the present moment, which in that regard was the world of Shiba Ryōtarō. In this sense, the rhythm of the mind and body are correlative with space, time, and presence.

The influence of the natural rhythms of a place are felt within the experience; this includes the flow of the trees as they slightly hit the glass wall, the sounds 


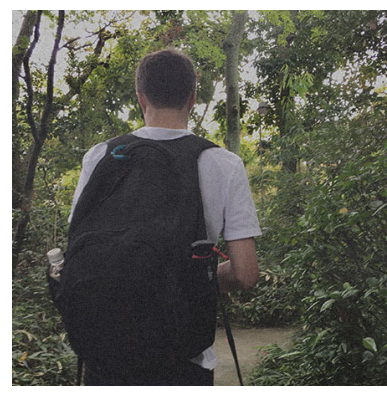

a

of branches rustling and the birds chirping in the background, and the shadow of the surrounding trees cast on the concrete floor.

A distinct rhythmic example is the use of thick black mullions at the museum's entrance. The mullions themselves along with their shadows begin to represent slices within the procession or moments in space and time. The rhythmic use of mullions heightens awareness within the mind and body to create a moment of pause within the procession.

One enters the museum through glass doors and follows a ramp towards the balcony which overlooks the bookshelves. It's difficult to describe the overwhelming feeling of both heaviness and lightness that takes over one's mind and body during an immensely moving experience. While standing at the edge of the balcony one feels enclosed by the books and their shelves. At the edge of the two spaces, a glass window floods the rooms with an abundance of light, and yet, the most prominent factor is that it's not broken into regimented panels, rather into panels of varying shape, size, and texture.

The main portion of the museum a $U$-stair is placed adjacent to the balcony; Ando's designs always consider the perceptual unfolding of an individual in space. With the museum, the main staircase is a U-stair rather than the typical L-stair in order to turn one in on themselves while descending the stairs. This elongates the procession since an L-stair would bring one directly into the centre of the space, however, a U-stair turns once on the landing and another towards the centre of the museum. This turning heightens anticipation and

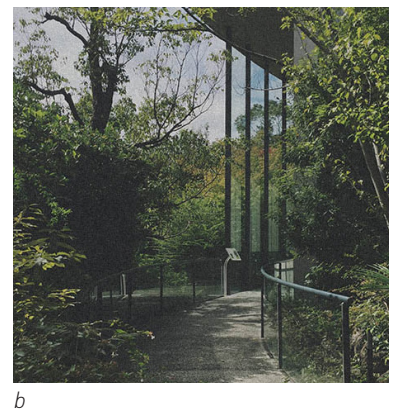

Shiba Ryōtarō Memorial Museum $2000-2001$

Tadao Ando

a_Trail

b_Promenade

C_Books

d_Stair

e_Pause

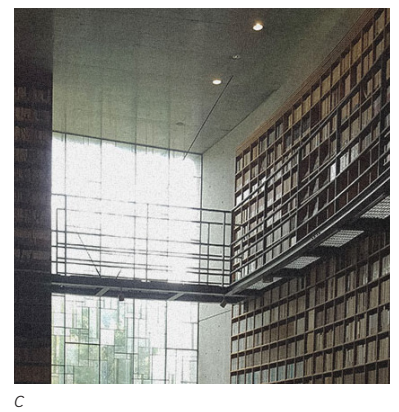



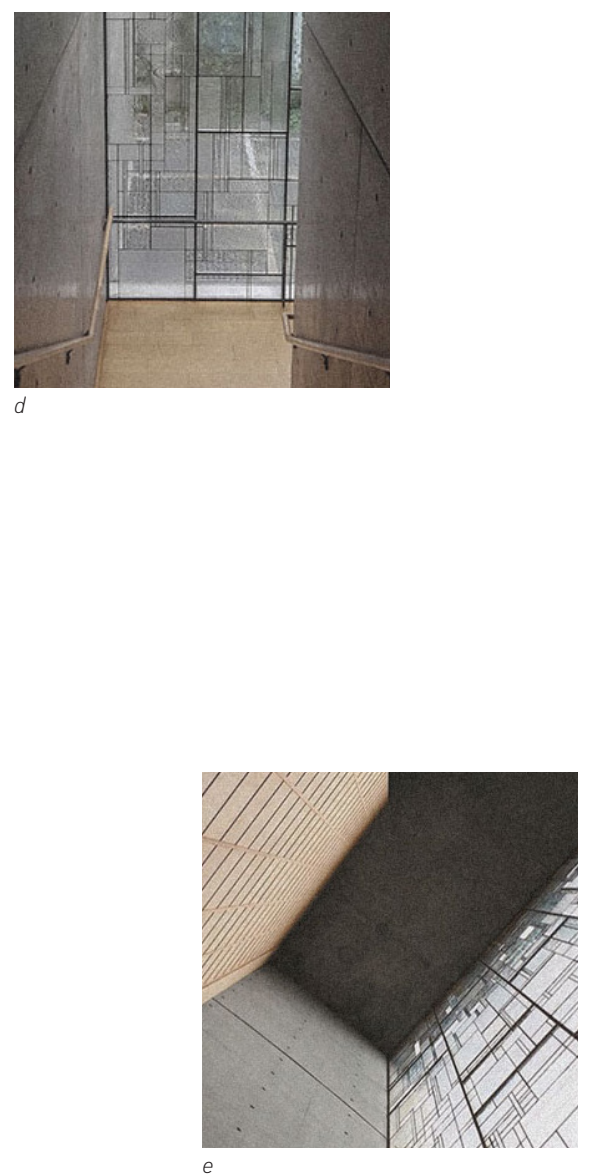

suspense, which inherently slows the procession while presencing the mind and body. In turn, making the next moment more impactful.

The immediate change of scale, after descending the stairs and turning towards the centre of the museum, creates a moment of pause. As one is surrounded by the enormous quantity of books and the grandness of the space the emphasis is placed on both Shiba Ryōtarō and his accomplishments.

After walking along the glass shelves and looking at various books one finds themselves standing behind the bookshelves looking up towards the large window and feeling very small. This emptiness and silence allows an individual to be still in the moment, losing track of time as a number but being fully aware of its passage. This pause is defined as ma in Japanese culture, a term which can be described as a gap or rest. It is within these pauses that one becomes both mentally and physically present in a place or moment.

Spatial proportions, namely compression and expansion, are influential generators of slowness since they change both the subject, the body, and the object, the space. With the scale, one becomes aware of the implications of material juxtaposition within a reading. The nook made visible the backs of the wooden bookshelves, the large window above, the concrete walls, and the wooden floor to create an empty space that contrasts the perceptual density of museum. 


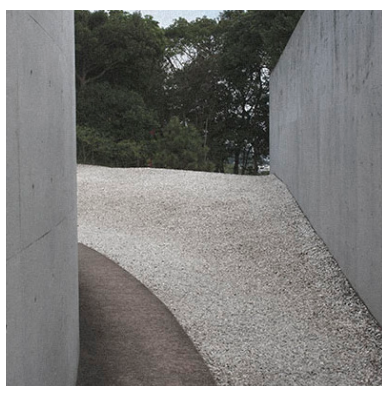

\section{The Water Temple}

The Water Temple by Tadao Ando is situated on the island of Awaji, just outside of Kobe, Japan. The entrance, subtly demarcated by a small red Torii in the distance, turns towards a large concrete wall with a single opening. Like the Memorial Museum, Ando is playing with anticipation by elongating the procession to the temple. This elongation is heightened by the nonlinear path by emphasizing the unknown.

Linear pathways condense time by revealing where one is headed, which hinges on the idea of looking into the near future; whereas, non-linear pathways expand time by limiting what is seen or what is to come, which allows one to inhabit both the past, present, and future simultaneously. Pathways with the end in sight are linear, whereas, pathways where the end is unknown are non-linear.

The large concrete wall stretches as far as the eye can see and contains a single opening. After moving through the threshold one is immediately turned and situated between two walls, the first was just passed through and the other curves away from view. While proceeding along the curved wall, the nonlinear pathway elongates the procession and begins to presence the mind and body. Gradually, the trees come into the foreground and the lily pond begins to reveal itself at the end of the curved wall. The turning positions the individual between the wall and the lily pond; by turning, the impact of the reveal of slowed and heightened. In other words, creating a moment of

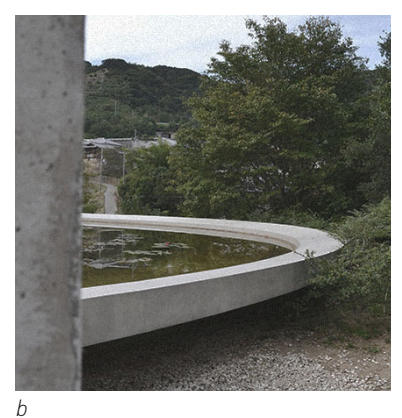

b

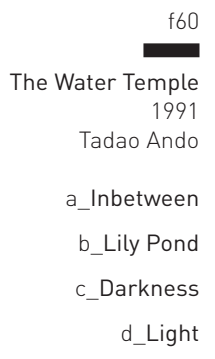



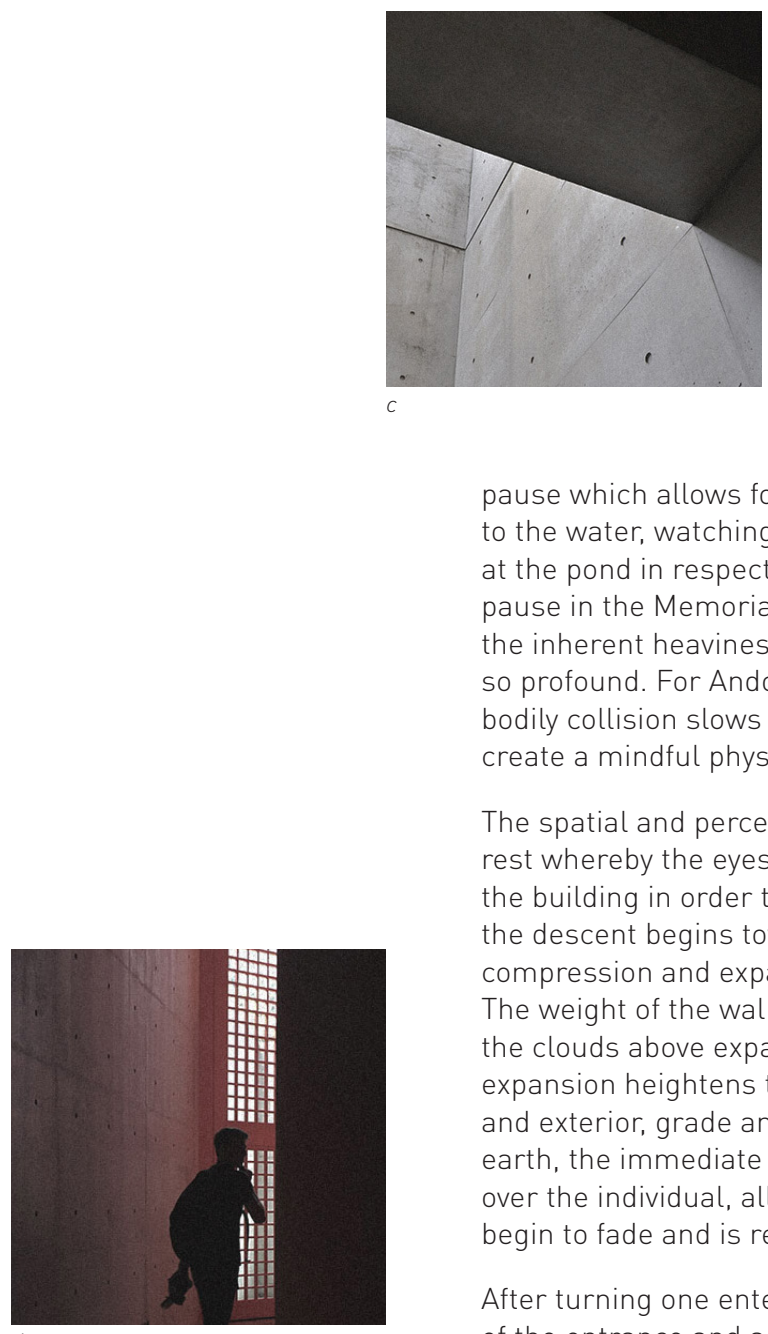

pause which allows for one to be present while listening to the water, watching the lily pads move, and looking at the pond in respect to the landscape. Similar to the pause in the Memorial Museum, it's hard to explain the inherent heaviness and lightness of an experience so profound. For Ando, the use of planar, spatial, and bodily collision slows the mind and body in order to create a mindful physical presence.

The spatial and perceptual layers implore a prolonged rest whereby the eyes travel from the landscape to the building in order to grasp their dialogue. Once the descent begins towards the temple a sense of compression and expansion overcomes the individual. The weight of the walls pushes in while the sky and the clouds above expands up; this compression and expansion heightens the transition between interior and exterior, grade and earth. As one descends into the earth, the immediate darkness of the temple washes over the individual, all the while the sounds of the pond begin to fade and is replaced by silence.

After turning one enters a dark space lit by the opening of the entrance and a red gated lightwell. As light filtered from both ends into the space the contrast of the pitch-black corridor towards the temple increased the felt compression within the space. A curving wall made of painted red wood moves one down a narrow but tall corridor into darkness; by compressing the individual spatially and perceptually one becomes increasingly aware of the mind and body. The corridor opens into the temple, which is a square room 


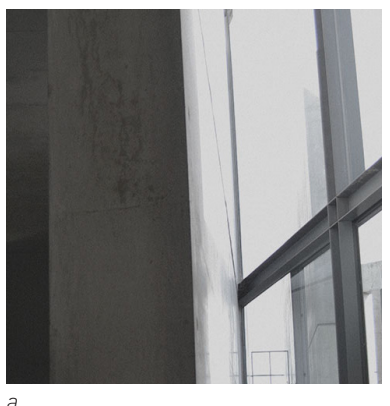

enclosing a golden Buddha that sits in front of the Western facing light well. A moment of pause is created as the light reflects off the Buddha and bounces around to highlight the red wooden walls and the dark corridor.

After leaving the Buddha one enters back into the dark corridor and continues the passage. Gradually, the corridor becomes brighter as the gated lightwell filters red light into the space until one finds themselves submersed within light. Similar to the Memorial Museum, the lightwell echoes the moment whereby the individual looks up to the large window in an empty space and becomes fully aware of themselves and the experience. The entire procession takes the individual from compression to expansion using both the spatial proportions and perceptual limits to presence the mind and body.

The Church of Light

The Church of Light by Tadao Ando is located in the small town of Ibaraki, just north of downtown Osaka, Japan. The intimate church blends into its surrounding residential architecture due to Ando's consideration of the neighbouring buildings. The entrance, up a slight hill, turns one into a courtyard that connects the church and the Sunday school; within the exterior space planar collision between the two buildings rhythmically moves the eyes.

After receiving a ticket in the small office of the Sunday school one moves through a glass opening alongside a plane towards the nave of the church. The entrance

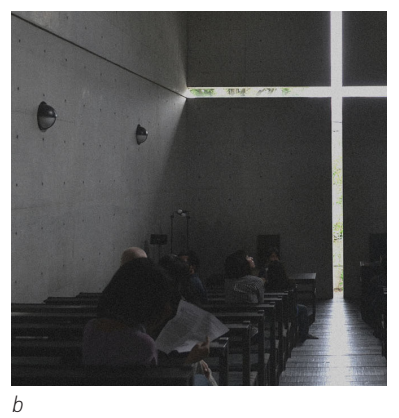



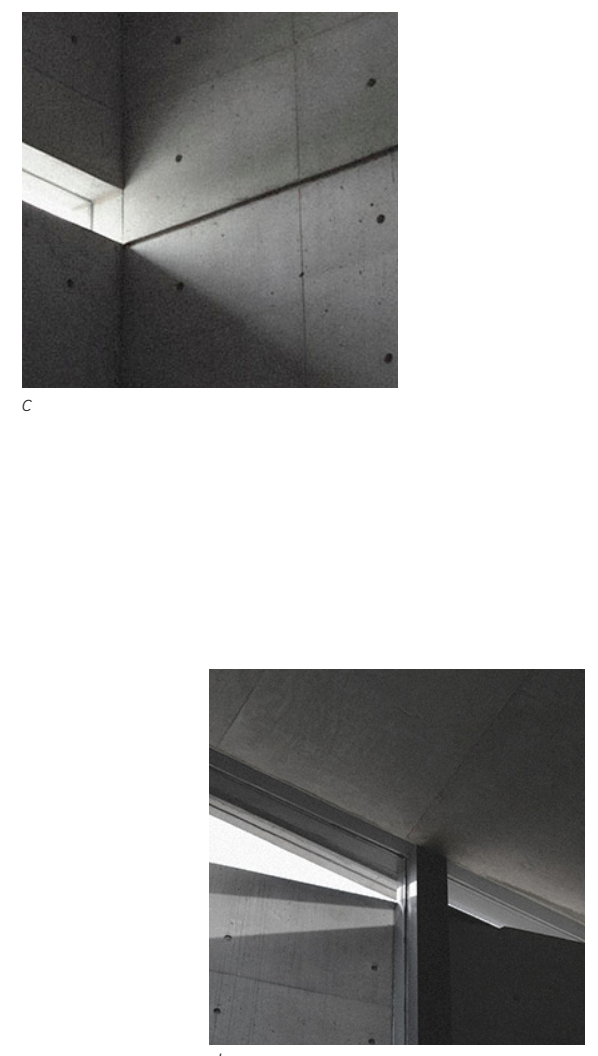

forces the individual to turn towards the nave to slow the reveal of its concrete walls, the wooden benches and the cross. The threshold between the interior and exterior is brightly lit to contrast the darkness afterwards where the church is solely lit by the cross behind the altar and the courtyard between its concrete shell and the entrance plane.

Once standing in front of the organ, looking down at the cross, it is clear that the scale of the church resembles that of the Water Temple. Unlike the Memorial Museum, which expanded vertically to presence an individual, the intimacy of the scale of the church and the temple associates one to something bigger than themselves, be it the light or a spiritual figure, one begins to feel connected to both the space and the experience. As one continues towards the altar the stepped nave begins to presence the mind and body with the physical concentration and exertion associated with ascending and descending a staircase.

Sitting at the back, one can take in the design and make correlations with the previous Ando projects; one obvious connection is the clerestory window, which filters light into the space while concealing the window, was also used in the same manner at the Memorial Museum. The longer one stays still and concentrates on a specific place or thing the more is seen; this concept is defined as slow looking and was previously discussed in association with the Slow movement. Stemming from 


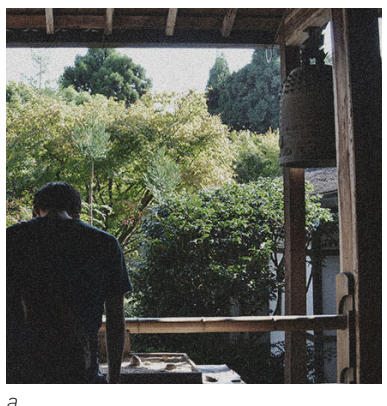

the Heideggerian idea of presencing, concentration permits the conscious and viscous experience of a thing. Both concepts rely on one's presence within an experience to allow for temporal inhabitation.

Three hours spent in the church and the Sunday school includes sitting at varying spots, moving up and down the nave, and watching others. The more time spent in the church the more aware one becomes of the subtle changes in the space: From the light and shadows shifting with each passing car or the movement of the sun to the sounds of the wooden floors creaking with each step. The church defines a place of silence, both mentally and physically, whereby the shifts in nature are seen within the concrete shell.

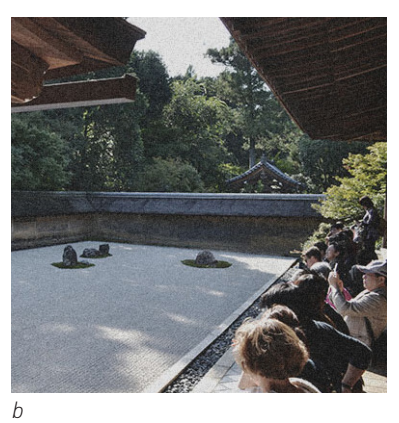

\section{Ryōan-Ji Temple and Rock Garden}

The following temples and gardens were experienced in Kyoto, Japan; the first was the. The procession begins with the experience of a large pond and various small gardens dispersed in order to guide you to the stone entrance of the temple, which is shaded by numerous trees surrounding the stairs.

As one proceeds towards into the temple and towards the rock garden, the spatial composition weaves the individual between interior and exterior space. This weaving includes opening, not typically glazed but rather directly open to the exterior; the integration between the building and the landscape is pertinent in slowing the mind and body since it creates moments of pause in which contemplation and meditation can occur within the procession. 


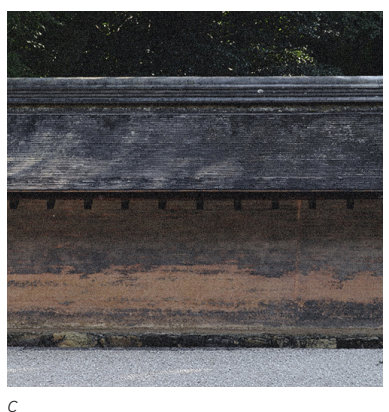

The rock garden is made of fifteen rocks dispersed in varying ways, on grass beds or directly placed, on raked sand. It is surrounded on two sides by an oil soaked clay wall that has seeped oil over time, creating a unique and varying pattern on each wall. The Japanese consider this to be wabi-sabi, a traditional aesthetic that accepts transience and imperfection, this includes asymmetry, roughness, and aging.

The many layers of the rock garden allow one to sit for prolonged periods of time concentrating on the mind and the body. The layers of the garden unfold like a painting; they begin with the foreground, the pebbles directly below one's feet, the middle ground, the sand and rocks, and the background, the wall and trees directly behind the garden. As one examines all the present layers, the past gradually reveals itself.

Although there is a lot of activity occurring at the rock garden, and yet, it is faded into a gentle murmur as one sits and experiences the rocks, the sand, and the clay wall. This type of experience is a silence more internal than the environment, it disassociates the individual from the noise and commotion to heighten concentration and awareness on the present moment.

After experiencing the rock garden one can explore the corridors and spaces throughout the temple. The fluidity between inside and outside creates slices through time, in turn, the spatial and perceptual cuts, allow quite dense, presence the individual in a corridor that would be otherwise seen as long and monotonous. The weaving allows for a montaged procession, which 


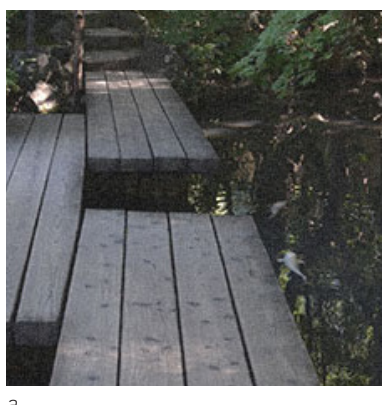

is understood as the layering of temporal slices. In this sense, the perceptual and spatial variety, if not overwhelming, can also slow a procession and presence the mind and body.

Nanzen-ji Temple

The following garden was the Tenjuan Garden of the Nanzen-ji Temple. A path of stones guides the individual past a sand garden and modest temple towards the lush garden and rock pond. The Tenjuan Garden is not one of the most famous gardens in Kyoto, as such, there is less visitors and activity at the garden; in turn, the experience is more serene and intimate.

After visiting traditional Japanese temples and gardens the influence it has on Ando's architecture is quite prevalent. By turning the body in on itself Ando rarely designs with straight pathways, he presences the mind and body by gradually unfolding spaces. This unfolding is pertinent in the design of traditional Japanese architecture, temples, and gardens, specifically the Tenjuan Garden.

The stone path takes one to a wooden bridge over a pond surrounded by bamboo. The wooden bridge shifts twice before taking the individual to the second pond; this shift presences the mind and body by heightening the physical awareness associated with stepping from one plank to the next. This consciousness disconnects the individual from the everyday by focusing on the present moment, which in this case was the motion of stepping onto the shifted planks.

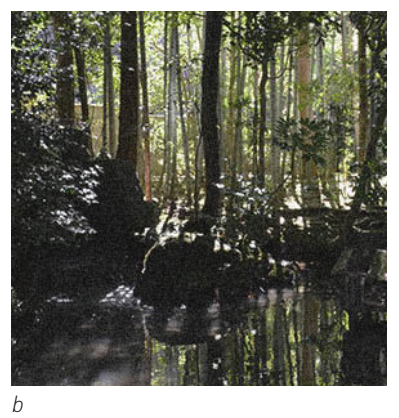




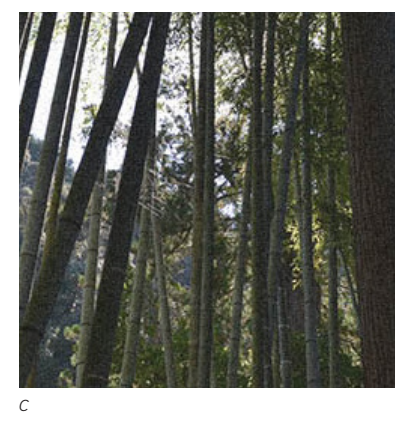

The atmospheric qualities of the first pond reference the Church of Light. Sitting on the stone stairs which lead to the wooden bridge allows one to take in the varying layers of the garden. From the mesmerizing temporal qualities of the water splashing from the tiny fountain, the koi fish swimming around the pond, to the dense bamboo wall filtering and flickering sunlight onto the pond. This prolonged looking creates a silence within the mind and body, one which creates a pause within the procession.

After passing the shifted bridge a stone path guides one alongside a small bamboo garden towards the stepping stone bridge. Similar to the stark shadows created by the mullions of the glass promenade in the Memorial Museum, the bamboo creates comparable slices of space and time through its shadows on the grass.

As one moves through the garden the other people in the space are seen through glimpses between the trees until they are lost in the canopy or darkness of the trees. Creating a rhythm between what is seen and what is concealed, in turn heightening the reveal of what is seen. Like the wooden bridge, the distance between the stones of the stepping stone bridge, although minimal, heightens a physical awareness by shifting focus towards the act of moving along the bridge. 


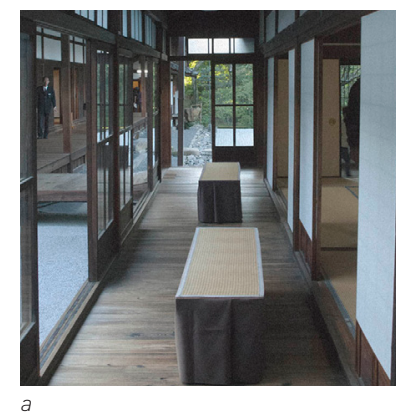

\section{Kennin-ji Temple}

The Kennin-ji Temple begins its procession at a linear corridor that looks, in the distance, at a sand garden. As previously experienced, the Japanese break up the monotony of corridors by exposing them to the exterior through light and openings. These moments, like a montage reel in film, heighten awareness in order to slow one's procession.

The procession towards the sand garden at the end of the corridor includes a smaller garden with a single tree in its centre. To the side is a wind chime that gradually flows with each gust of wind, echoing sounds as the elements of the chime clash together. Continuing down the corridor turns the individual towards a lush garden with several stones dispersed throughout it. The sun was beginning to set and cast a dappled light on both the garden and the temple walls.

Like Ryōan-Ji Temple and Rock Garden, the weaving in and out of interior and exterior space is prominent when slowing the mind and body. However, Kenninji used an interesting new form of weaving which included a covered pathway that moves an individual between two gardens into the next temple space. The different way of connecting these two buildings makes the individual feel completely immersed within the two

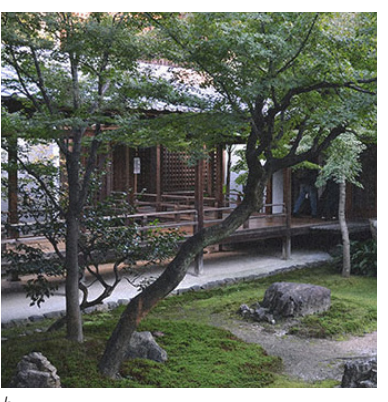
gardens.

Through the interplay between the natural and manmade elements, the Japanese have established an architecture which relies on concentration, presence, and awareness in order to slow the mind and body. Continuing down the corridor, people are seen sitting 


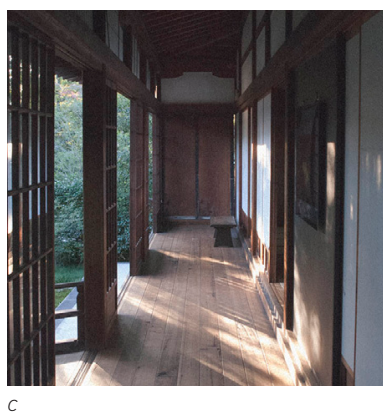

and gazing at the sand garden, watching the natural rhythms of the place change ever so slightly with each moment.

The temple creates an atmosphere for traversing and sitting while looking and feeling the varying elements within the experience. This level of interaction with the space highlights the consideration of materials, including how light will seep through shoji screens, if the view towards a garden is glazed or open, or the connection between varying wood joints. The overall integration between the natural and man-made environments develops an atmosphere of mindful physical presence. 


\section{Sequence 01 Appendix B}

The following appendix includes additional drawings and information for Sequence 01 of The House on the Coast. 


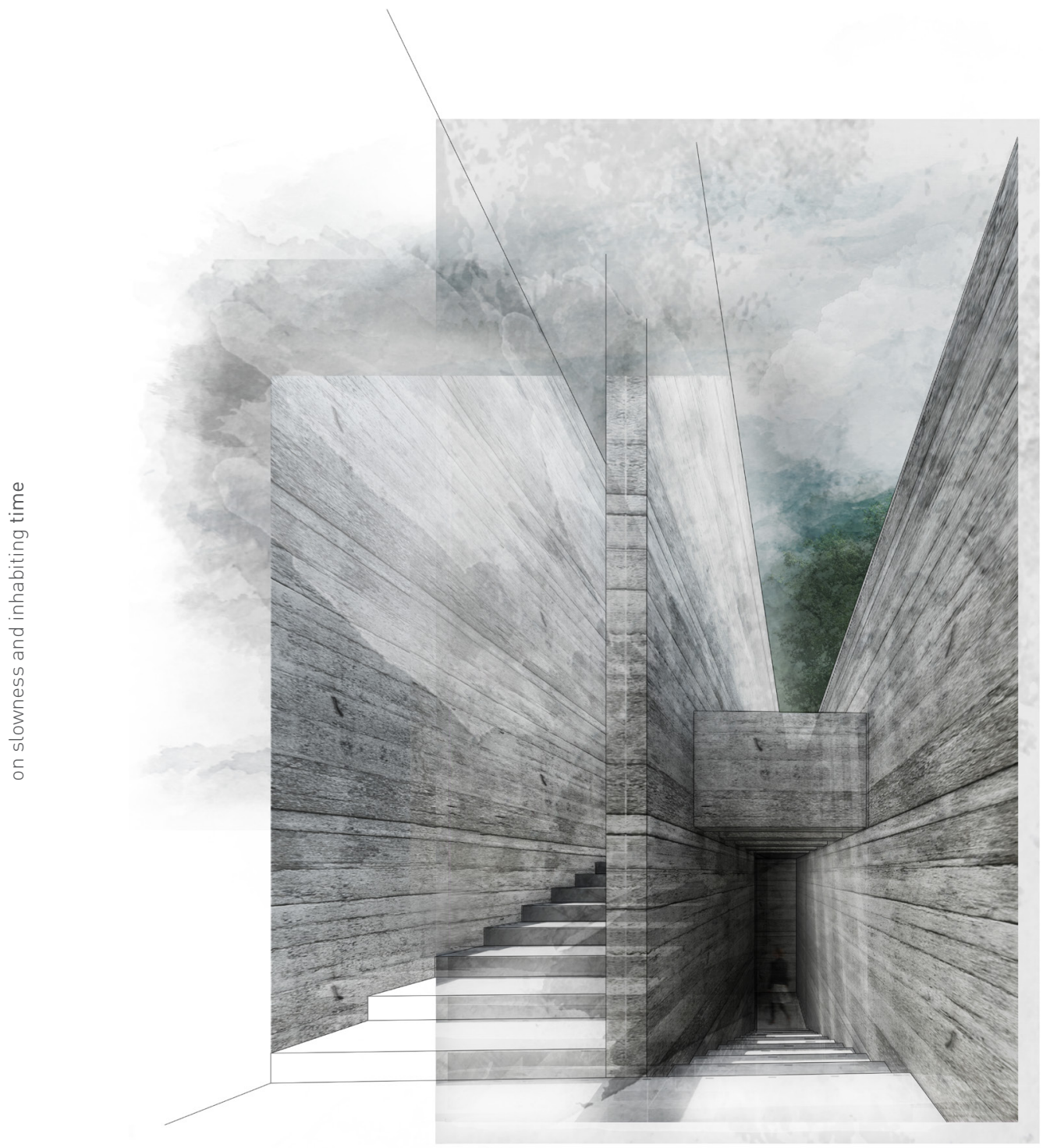

f65

Stills: Sequence 01

a_Entrance Steps

The inhabitant is turned and situated between two walls that look towards a dimly lit landing. The pergola above creates both a rhythmic descent into the darkness, as well as, temporal and spatial slices by casting shadows on the adjacent concrete wall and floor. The wall on the right angles towards a narrow landing, this change in proportion heightens awareness by compressing space. 


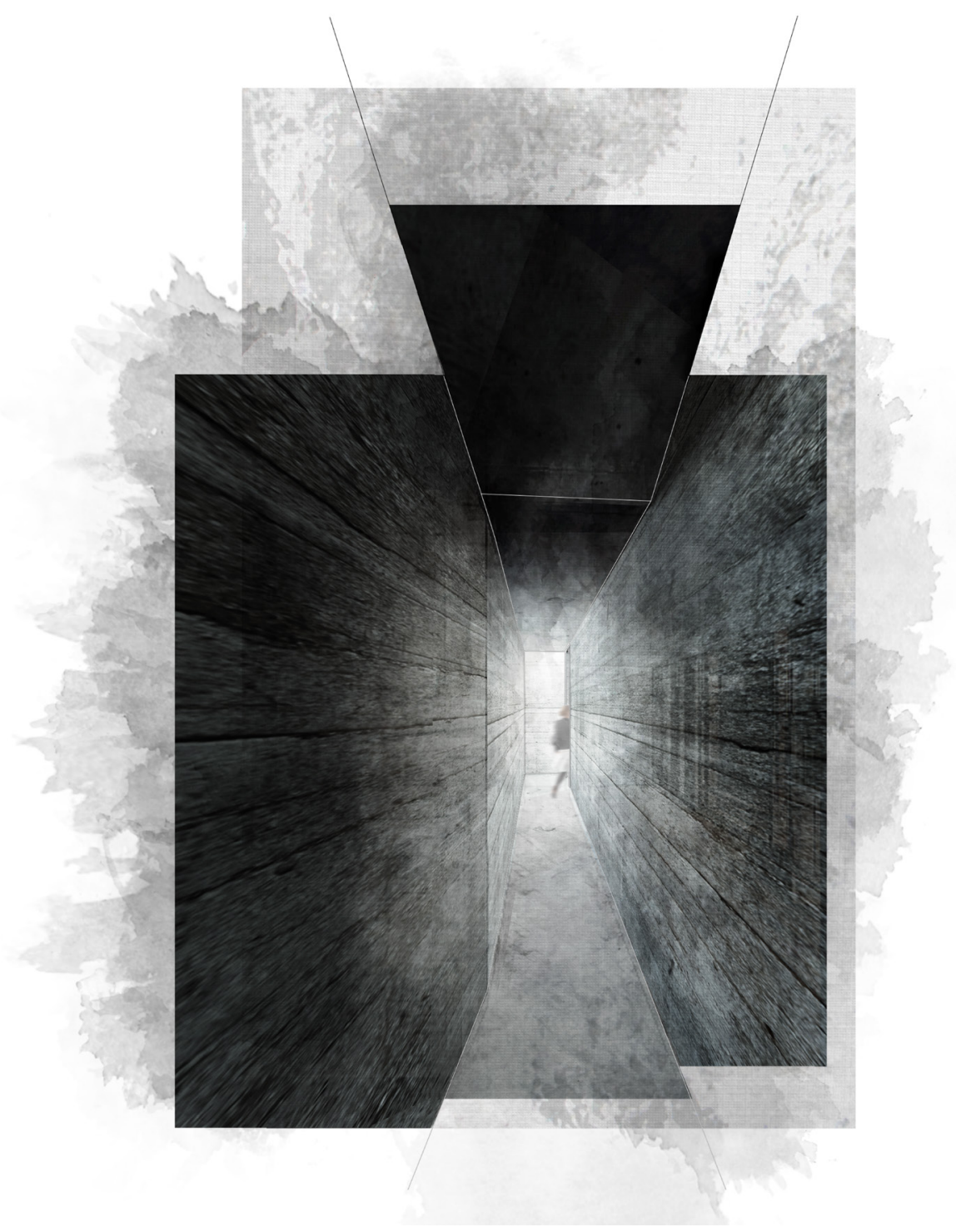

f65

Stills: Sequence 01

b_Through the Doorway

The inhabitant is briefly expanded at the landing as the descent continues down another set of angled stairs that compress towards a dimly lit corridor. The light from a single slit window at the end of the corridor guides the individual towards the entrance of the dwelling. The transition between open air, darkness, and light presences the mind and body by juxtaposing moments of atmospheric variance. 


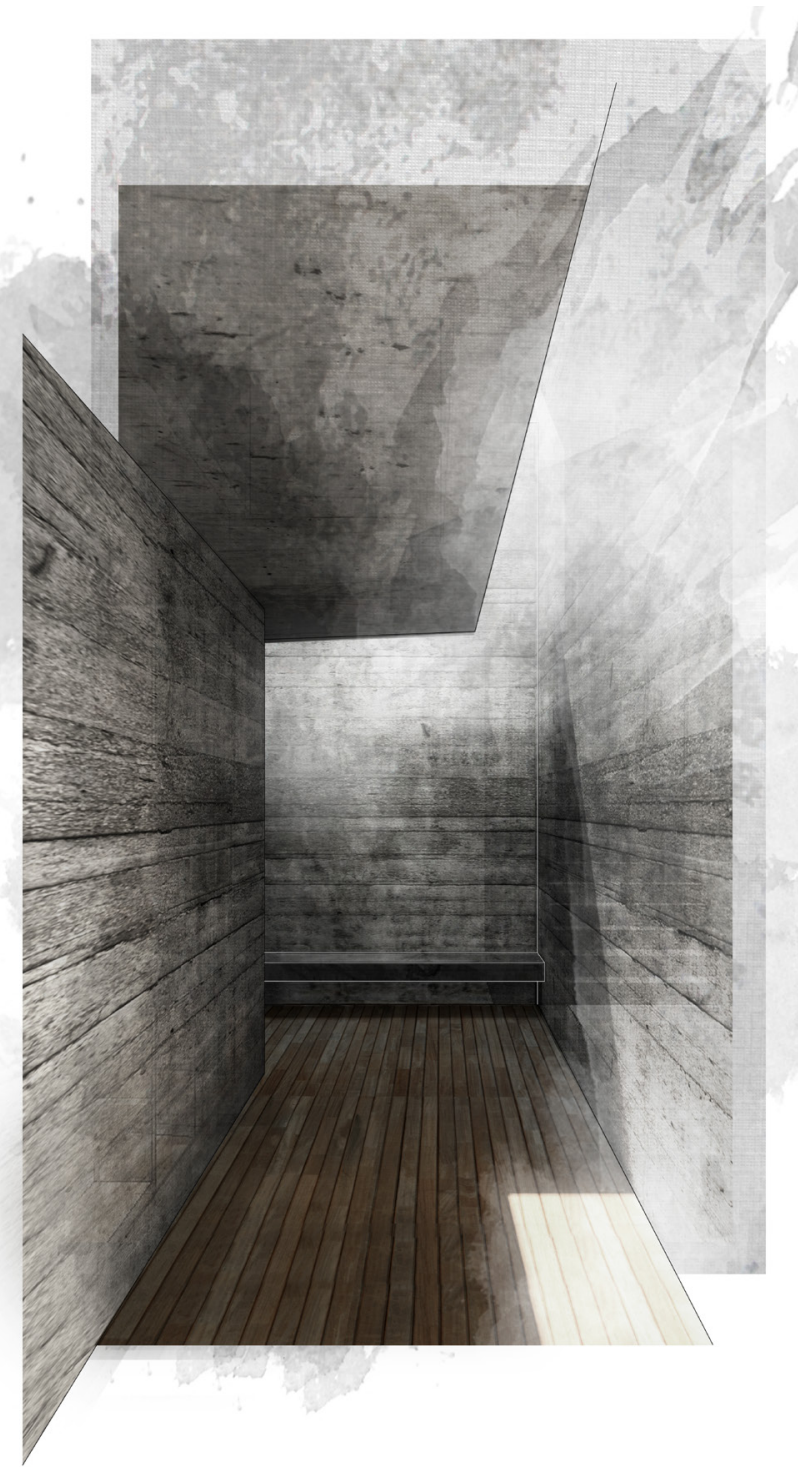

f65

Stills: Sequence 01

C_Foyer

A large concrete door acts as the threshold between the corridor and the entrance to the dwelling. After passing the threshold, one is immediately immersed into a space guided, both optically and bodily, by light. The non-linear passage elongates the procession by heightening anticipation and suspense of the unknown. The skylight is the first element that begins revealing the landscape to the inhabitant. 


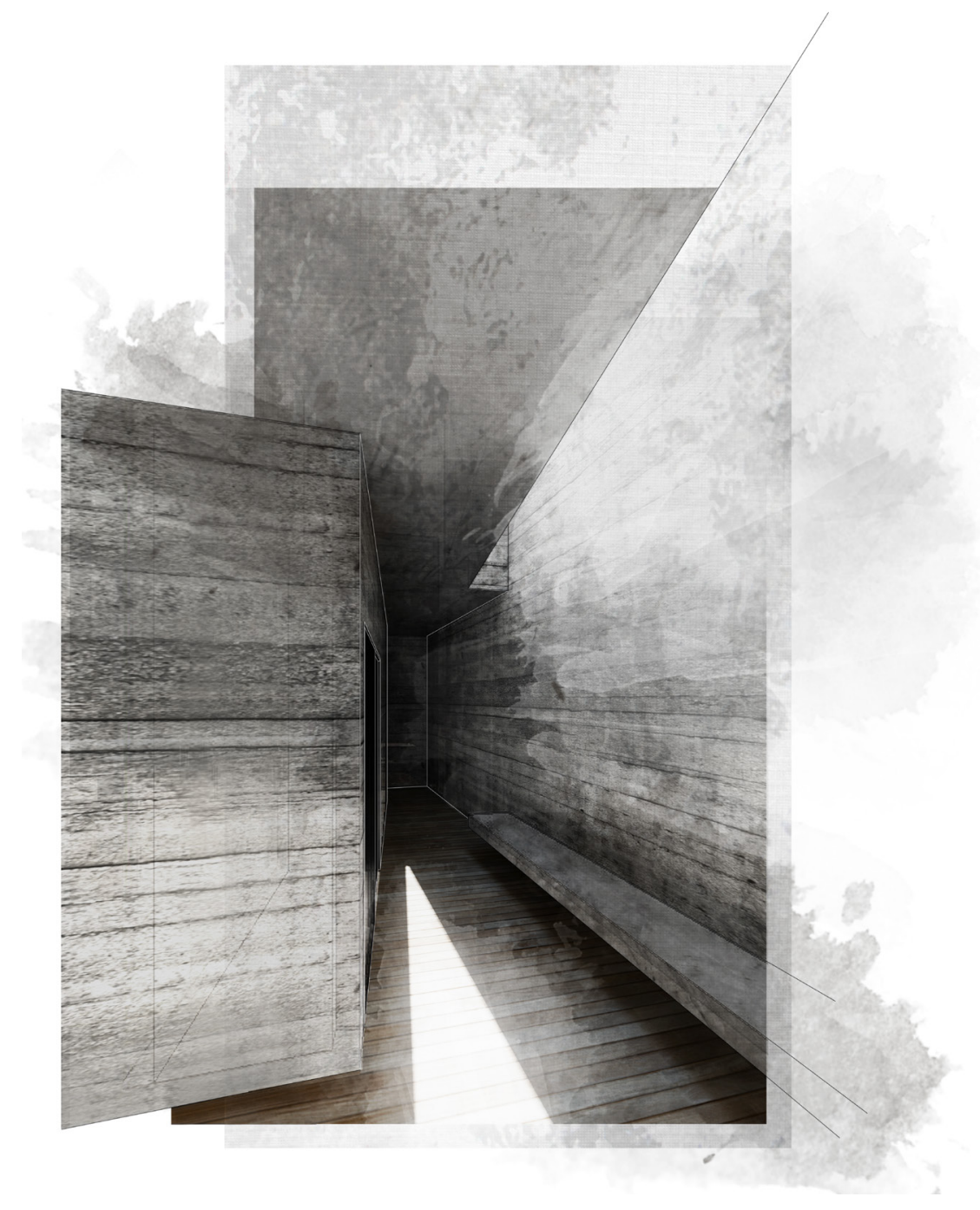

Stills: Sequence 01

\section{d_Bench Under the Skylight}

At the end of the hallway, one turns to find themselves between two varying walls; the one on the right is straight and lit by the skylight above while the other stretches further into the space and angles to create a compressed corridor. The angled wall moves one into a dimly lit space that extends into the following narrow corridor. 


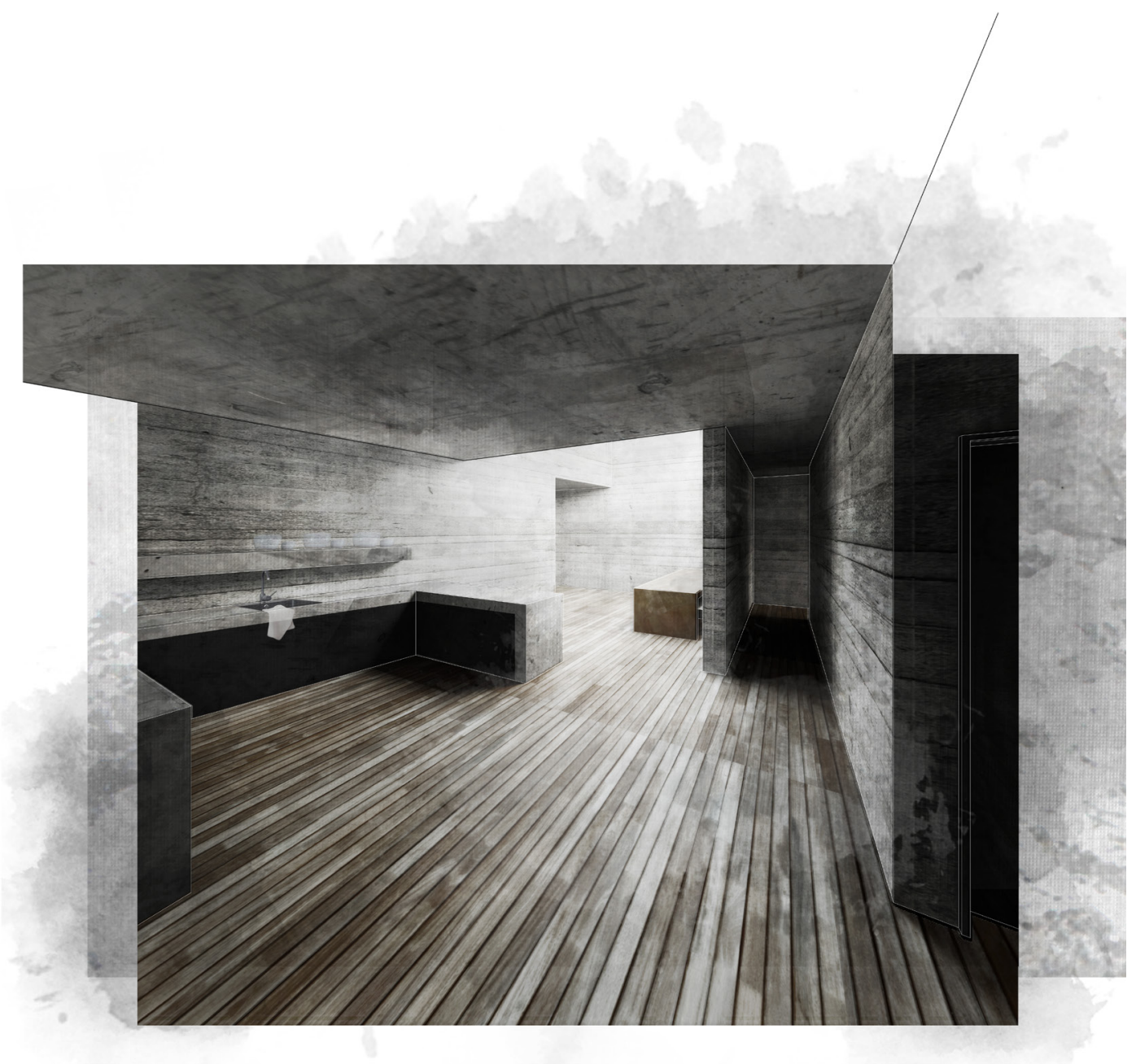

f65

Stills: Sequence 01

e_Kitchen and Dining Room

As one turns the corner, light pours in from a large skylight above the dining space; the skylight is the second element which begins revealing the landscape to the inhabitant. At this point, light has been reintroduced, thereby, the skylight allows for both light and sight of the sky, the stars, and trees above. 


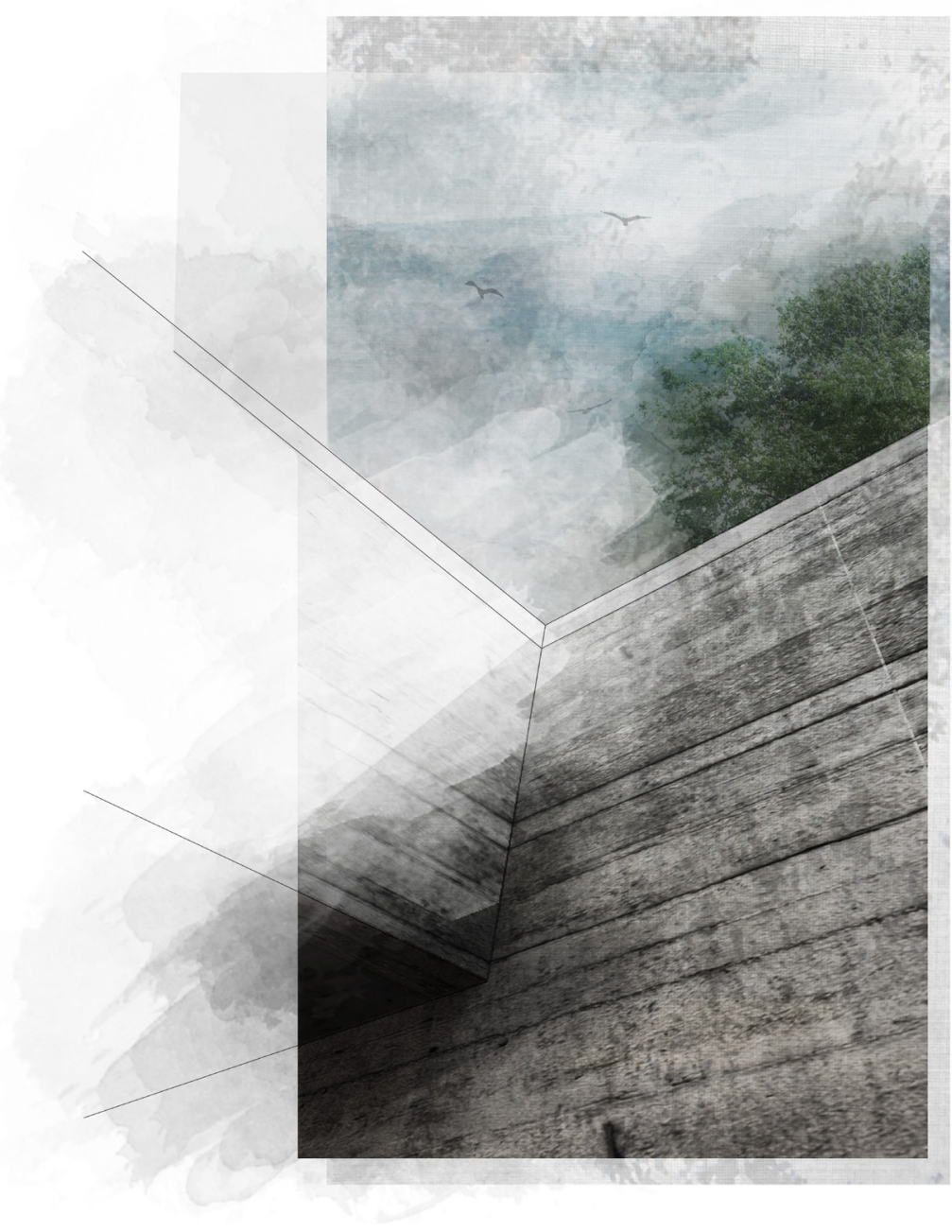

665

Stills: Sequence 01

f_Dining Room Skylight

As one walks towards the skylight, the heaviness of the walls, and lightness of the sky, consumes the individual. The space becomes a gap, an emptiness that allows one to be still in a specific space or moment; losing track of time but also being very aware of its passage. 


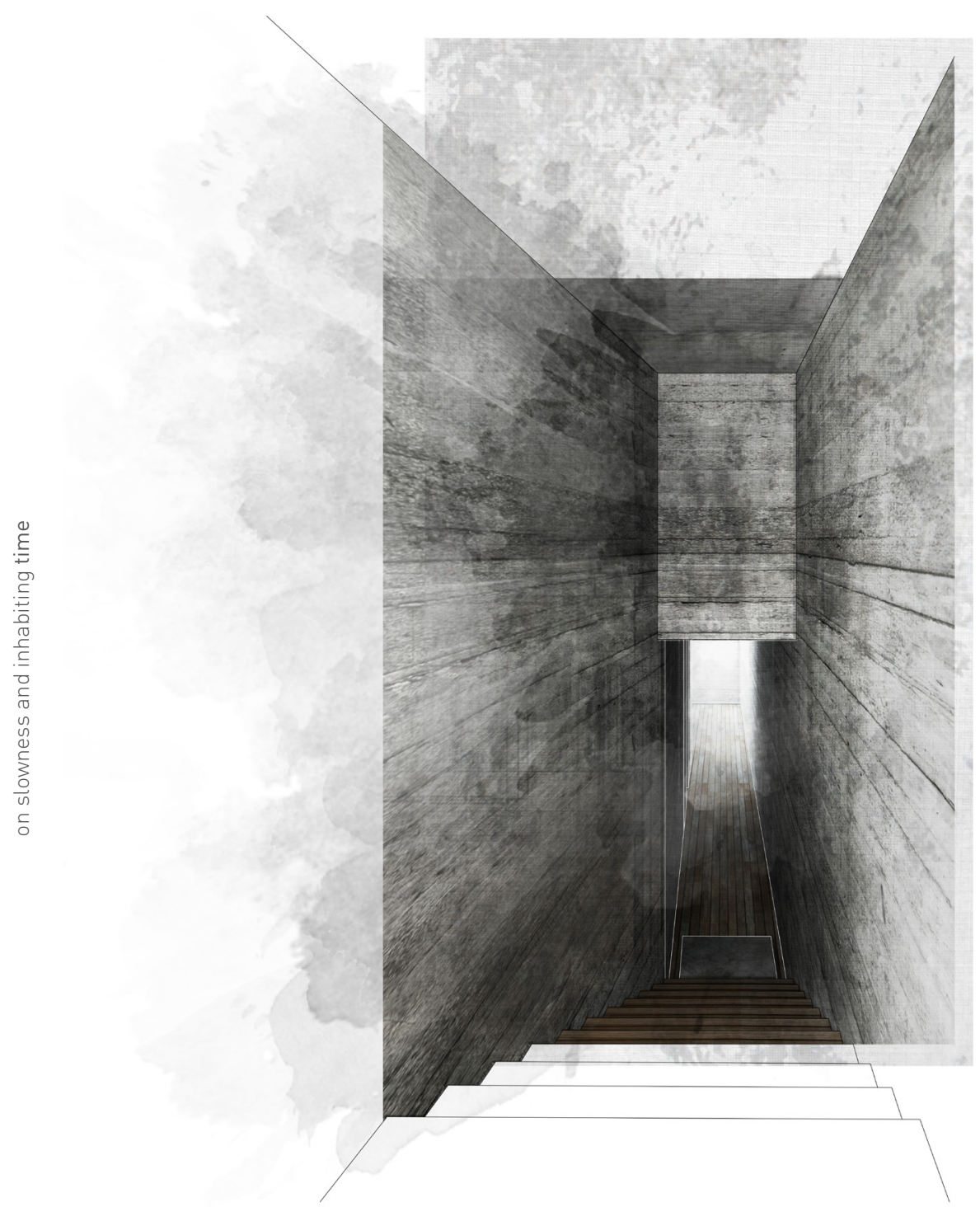

f65

Stills: Sequence 01

g_Compressed Stair

Turning the corner situates the inhabitant between two walls which compress towards a narrow corridor, both in plan and section. The stairs are wooden, like the floor, but the final step is disconnected and made of concrete. This irregularity within the rhythm of the procession creates a distinct moment of pause which presences the mind and body. 


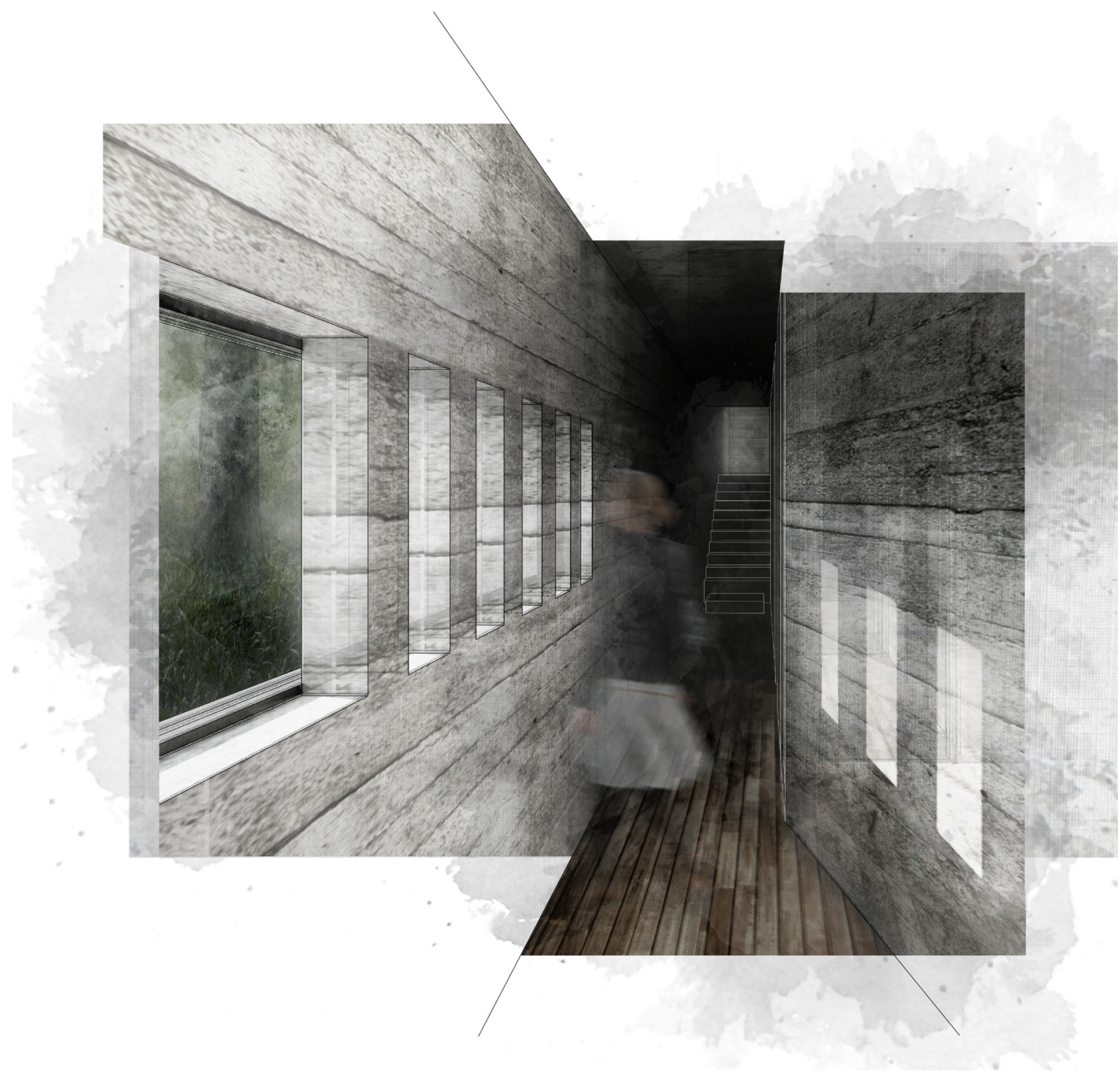

Stills: Sequence 01

h_Expansive View of Terrain

The wall on the left has three distinct openings, one that enters a separate hallway that links the bedrooms and the main bathroom, and two slit windows that create rhythmic slices in space and time. The wall angles to expand the space as one proceeds down the corridor. The horizontal window, within this expansive space, is used rhythmically to unfold the view of the tree trunks and terrain outside. 


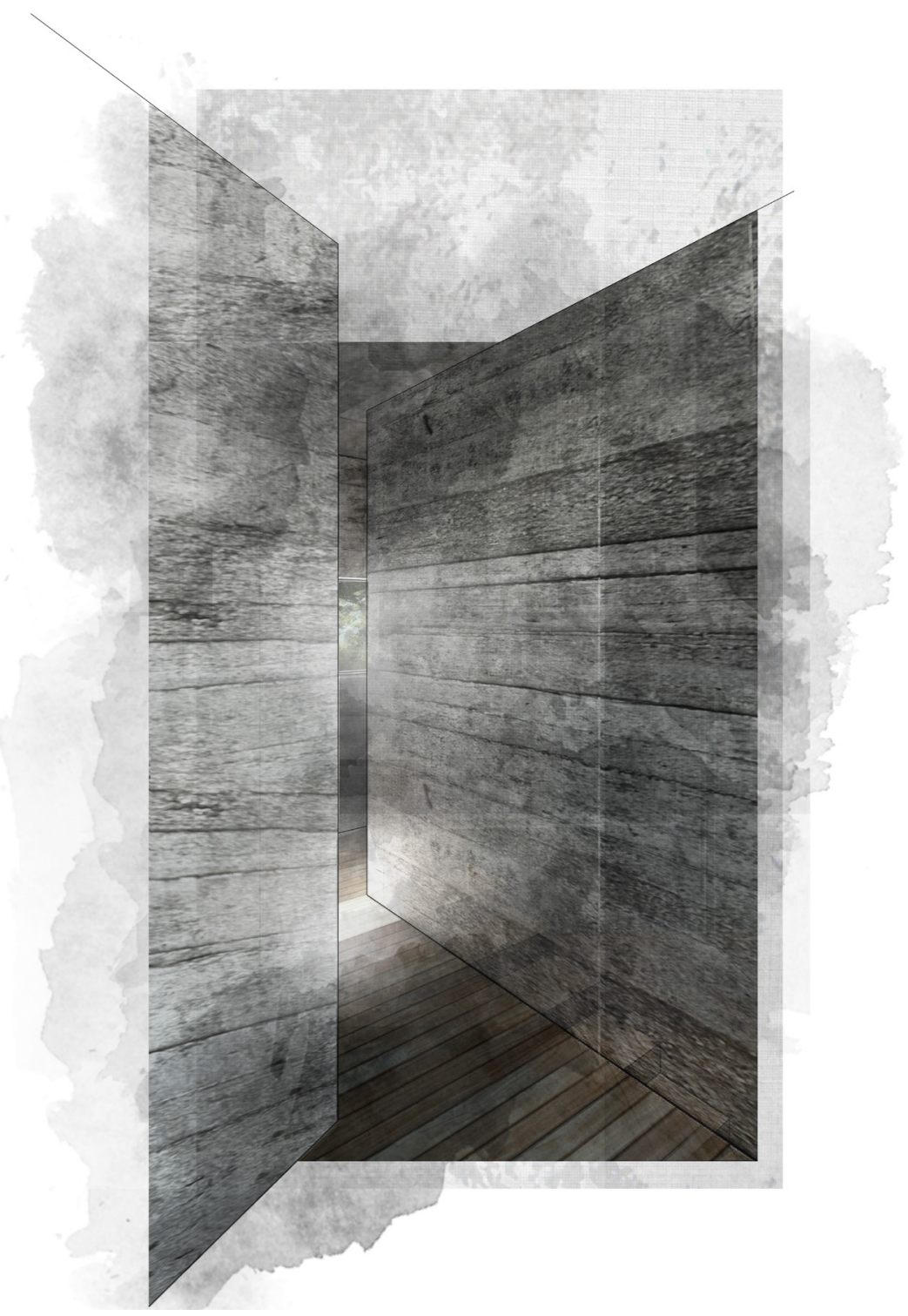

665

Stills: Sequence 01

i_Last Bend

The window opposite to the one previously mentioned creates an axis that allows for the inhabitant to look from one side of the landscape to the next; both at the level of the tree trunks and terrain. The expanded space compresses into a narrow corridor which guides the individual towards a stair, similar to the previous one. 


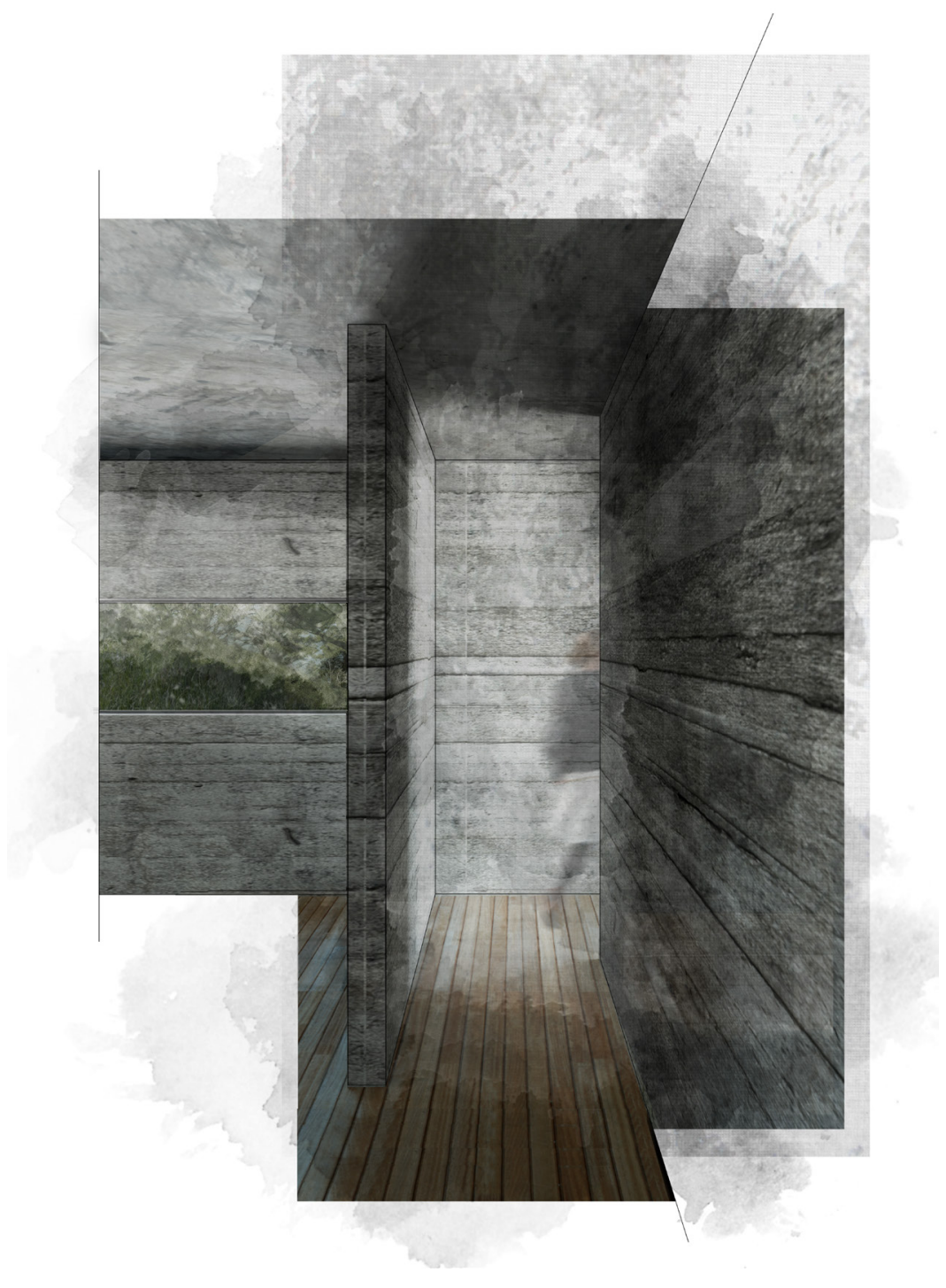

f65

Stills: Sequence 01

j_Living Space Corridor

The stair turns the body once before continuing straight towards a concrete wall. Following the final step, the non-linear path turns the individual one more time in order to situate them between two walls, whereby, the horizontal window within the first living space highlights views of the terrain 


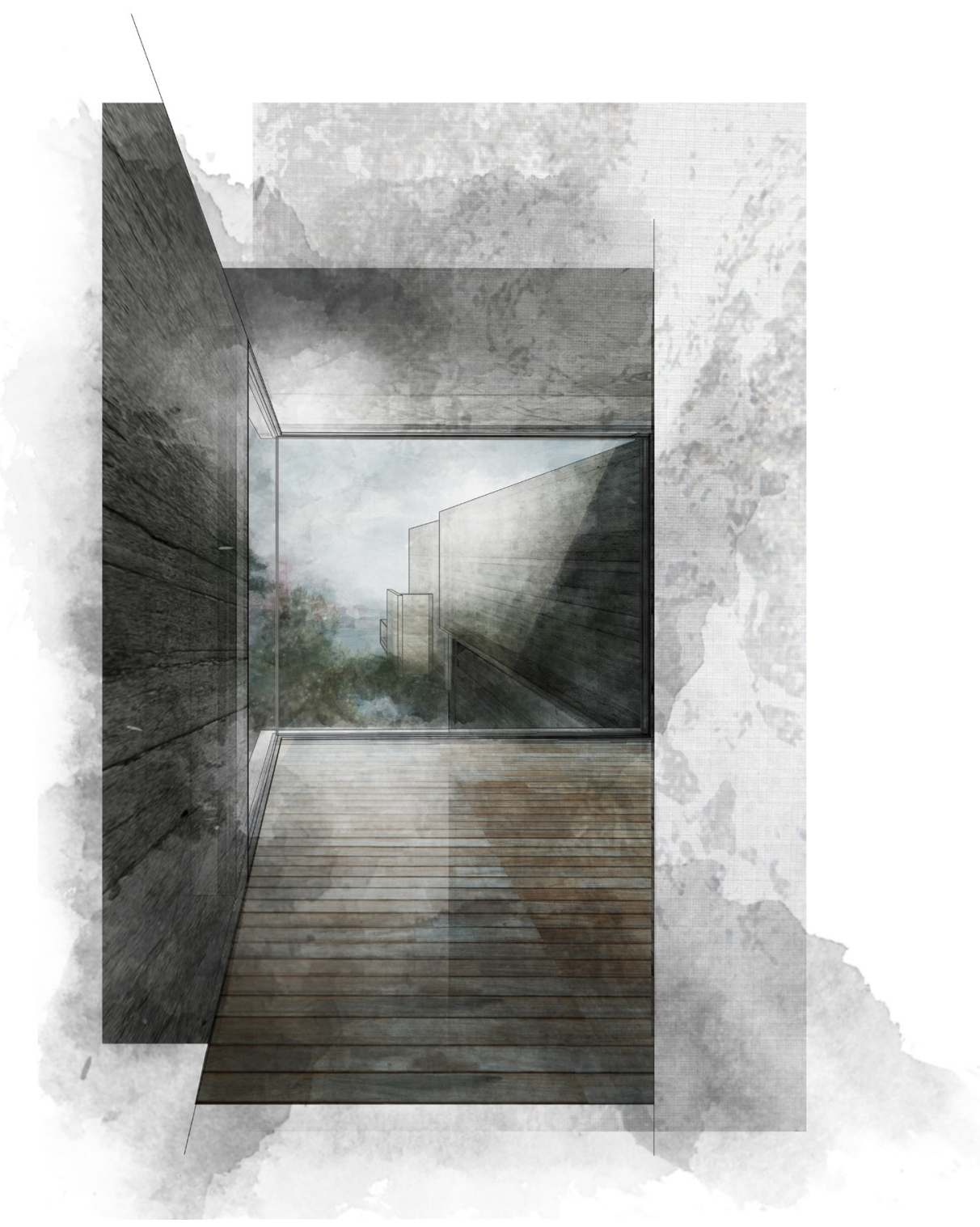

f65

Stills: Sequence 01

k_Living Space View

One continues down the narrow corridor, towards the light and turns to discover the landscape in all its entirety. The non-linear path elongates the procession by heightening anticipation and suspense; in turn slowing the experience for the inhabitant by creating overwhelming moments that presence the mind and body. After one step, one continues towards the final space: the space of silence. 


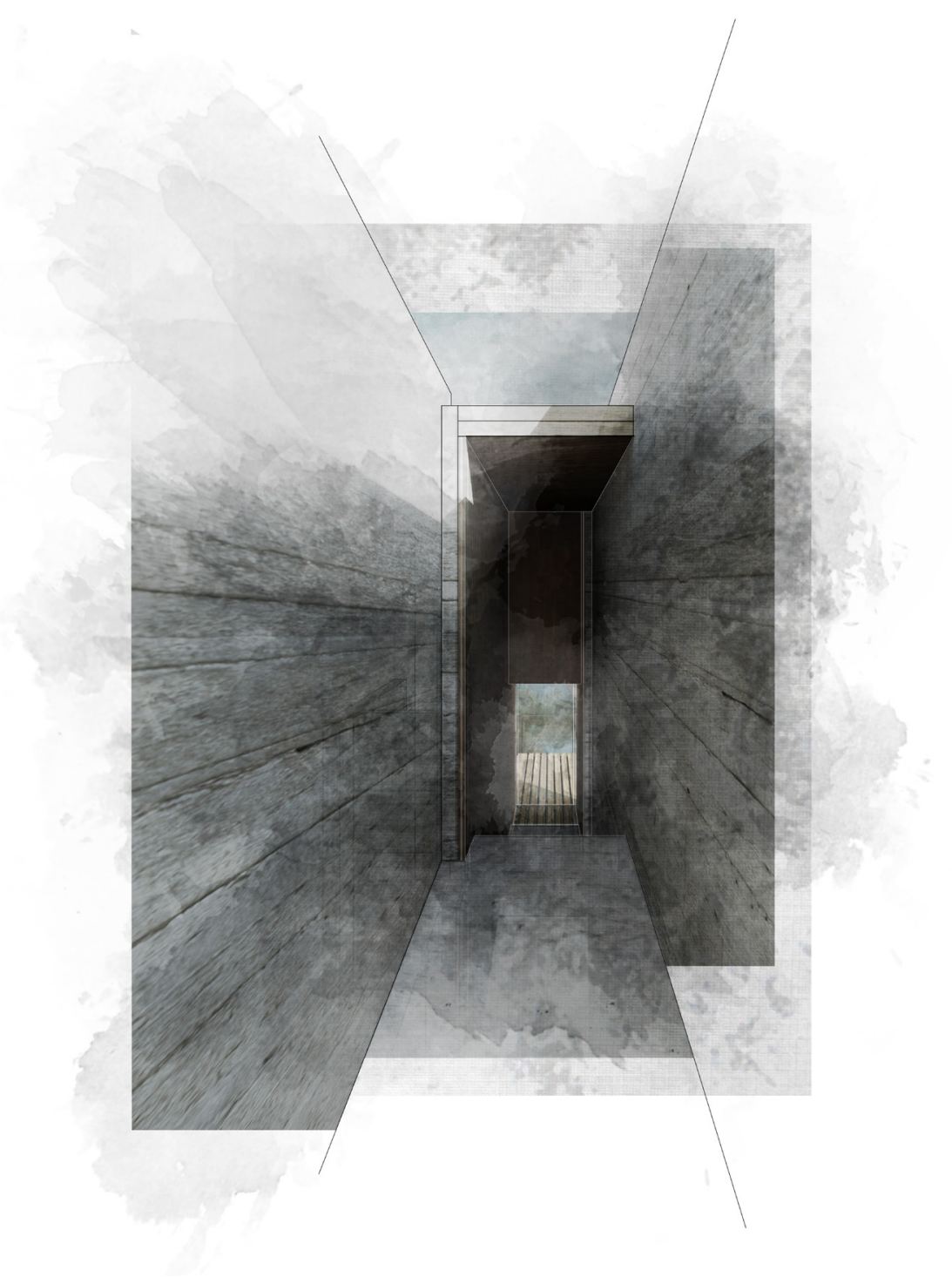

665

Stills: Sequence 01

L_Towards Silence

Throughout the procession moments of pause have been considered as expanded moments within space and time. The final space within the dwelling is of silence. Silence, unlike pause, is an end - it is the enmeshed experience of duration, presence, and place. Once through the concrete threshold, one is situated between two walls, which compress in plan but expand with the open-air section. 


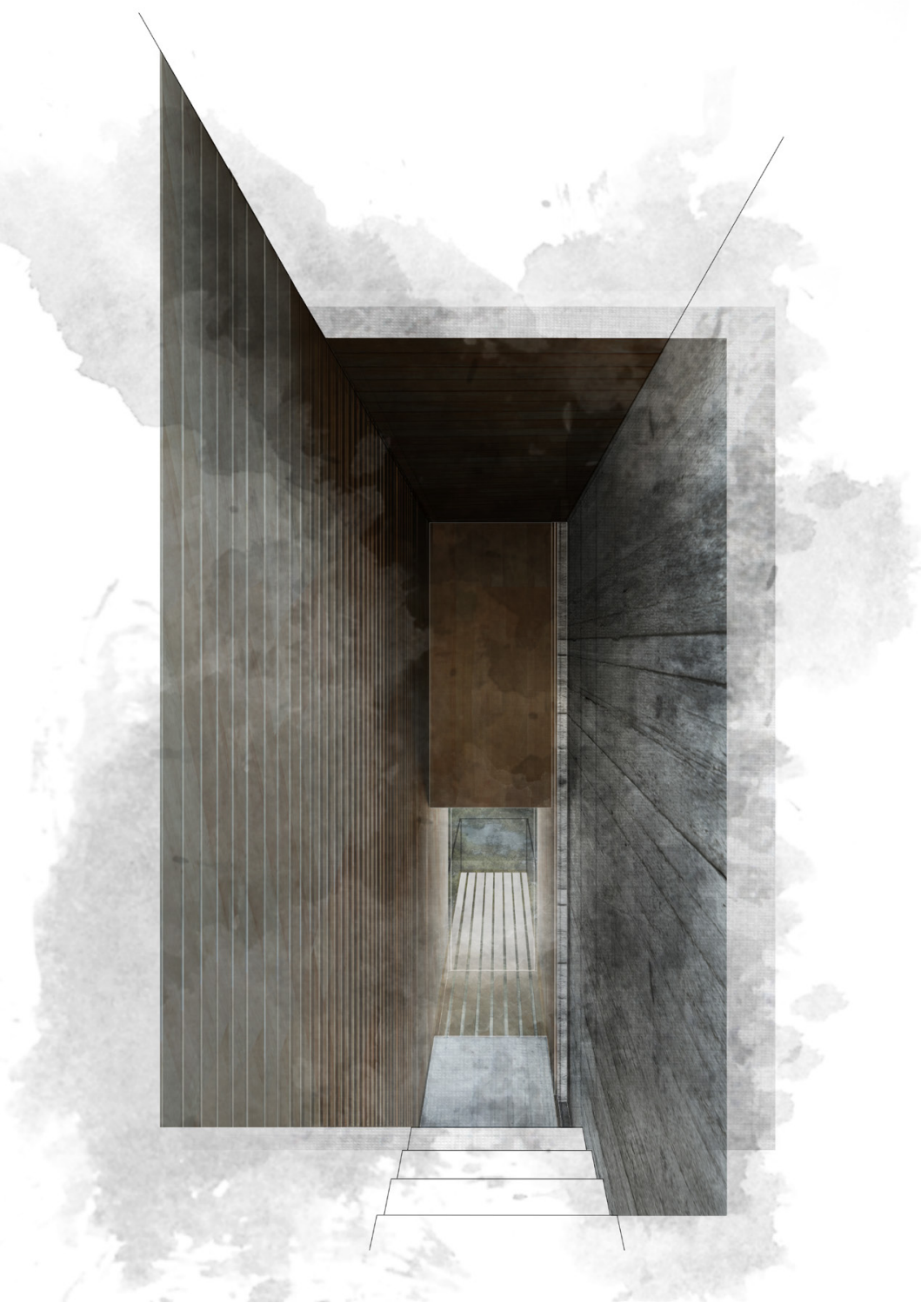

f65

Stills: Sequence 01

m_Seat Towards Silence

Unlike the board formed concrete on the right wall, the introduction of wood on the left wall is placed vertically to create a clear contrast between spaces for pause and silence. The space of silence is an end, as such, it is no longer necessary to use the wall to guide the inhabitant through the procession. 


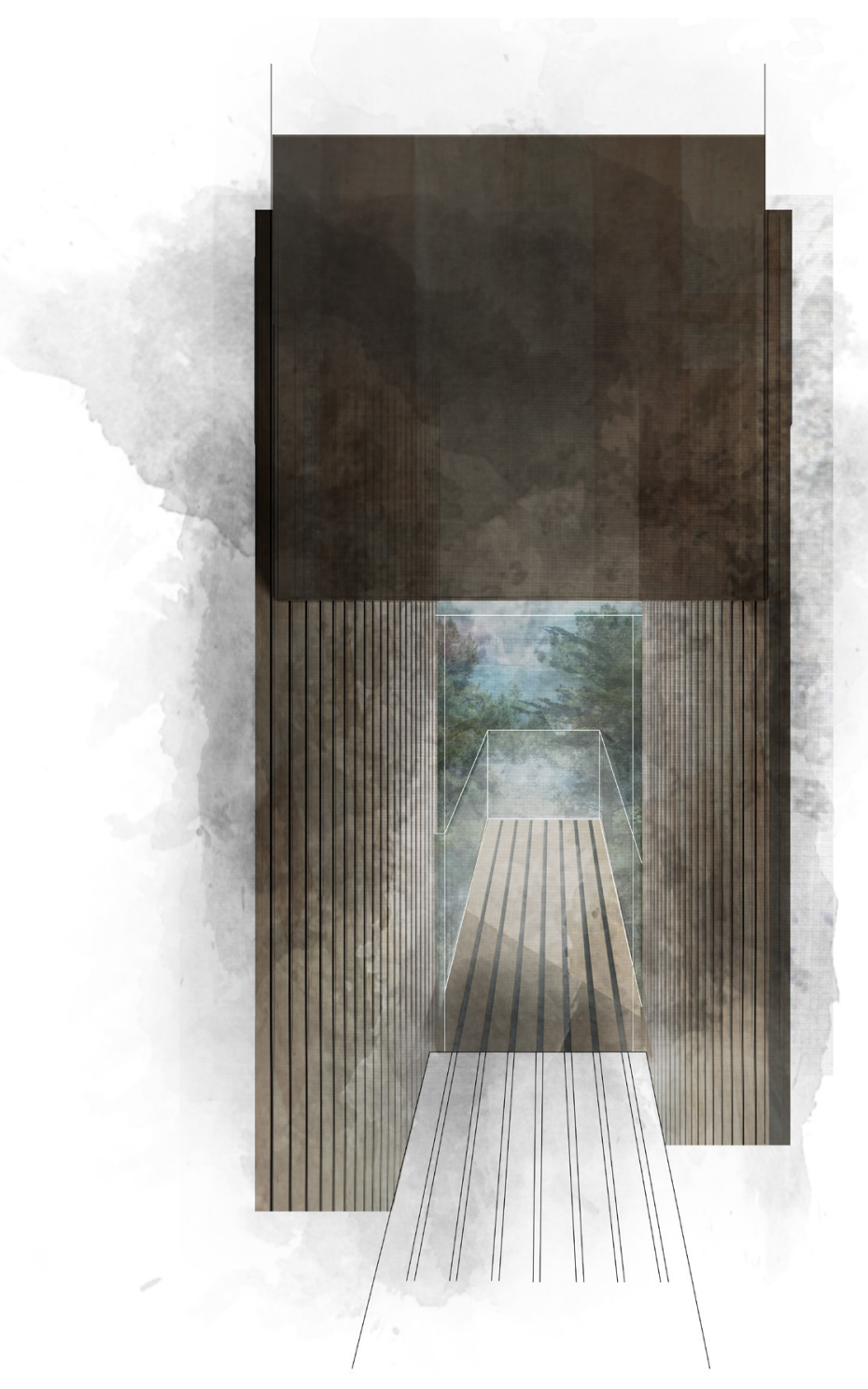

Stills: Sequence 01

n_Sit in Silence

Similar to the previous stairs, with the last staircase, the final step extends to create a seat, which further encapsulate a moment of silence within the procession. The seat signifies an expanded present whereby stillness creates temporal inhabitation. 


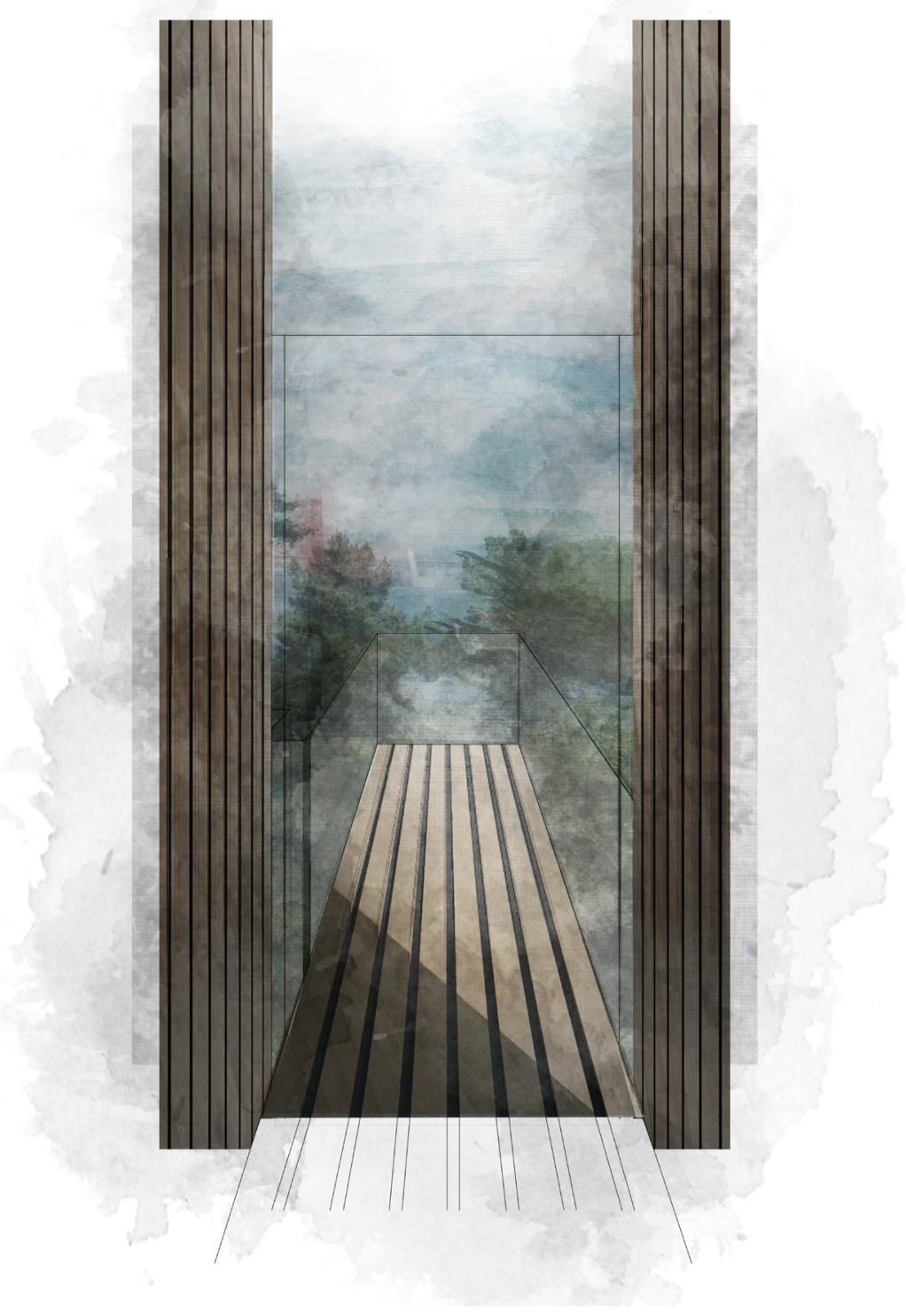

f65

Stills: Sequence 01

o_Threshold Between Silence

The space of silence includes three different spaces for stillness: one being the seat at the end of the stair, the other being directly outside on the exterior extension, and finally a space which pulled back and hidden from any view. 
Stills: Sequence 01

p_The Moment of Silence

As one moves through the glass door, the individual steps behind the wall and is able to look up and out, towards the sky and water. Passing the threshold situates one above the trees looking out towards the water. This overwhelming feeling of being immersed within the landscape creates the final moment - the moment of silence. 

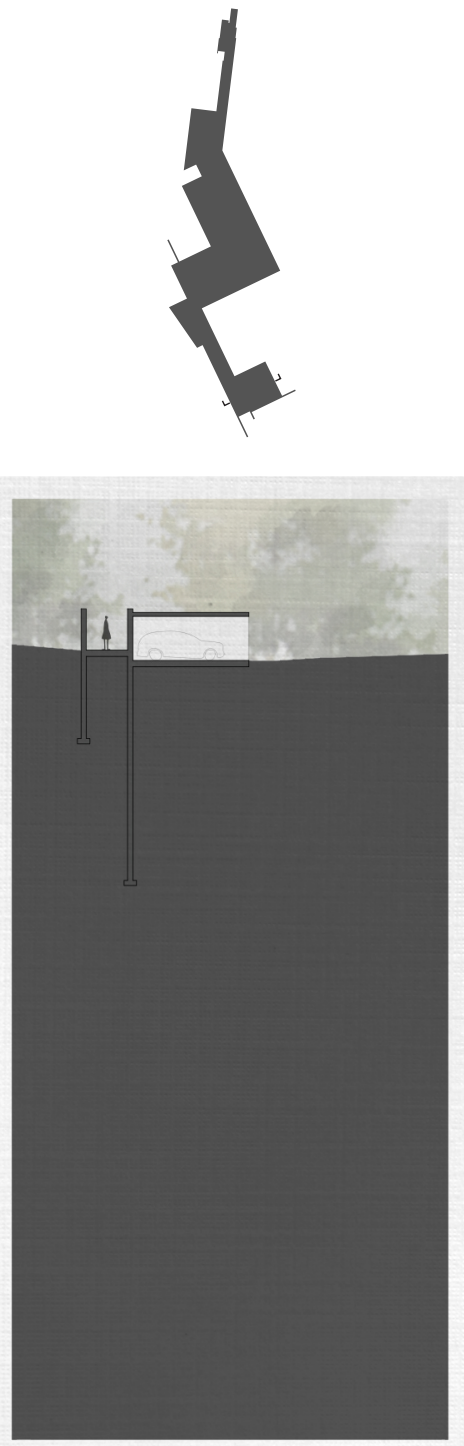

f51

Orthographic Drawings

g_Cross Section Study, Section aa and bb, Sequence 01

Referencing John Hejduk's cross sectional study for Wall House 01. This study intends to understand how various Generators of Slowness, including light, scale and proportion, can create a slowed procession that presences individuals both mentally and physically.
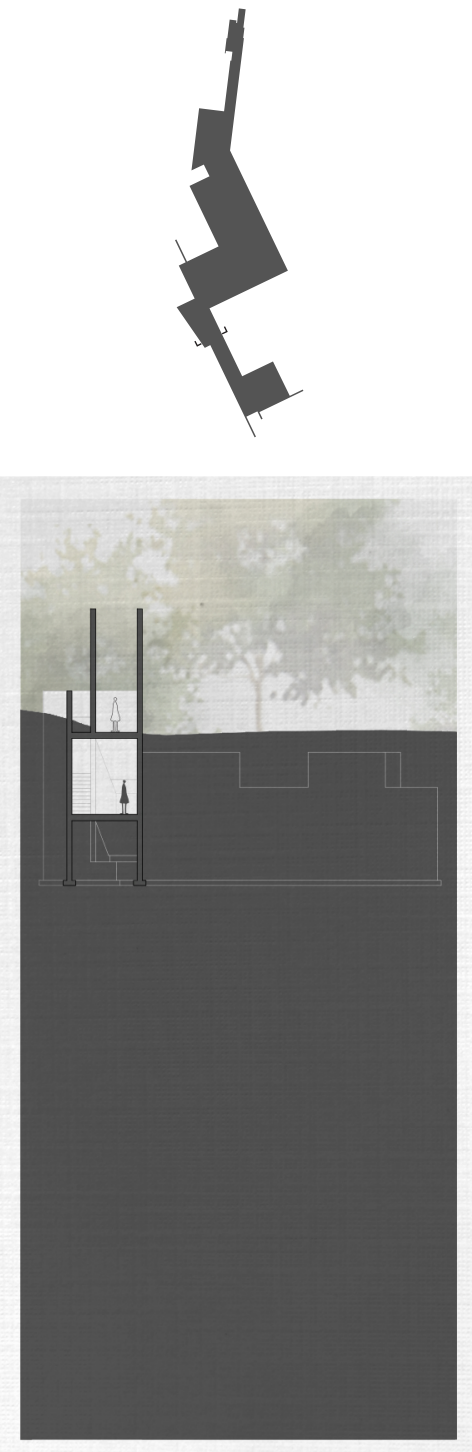

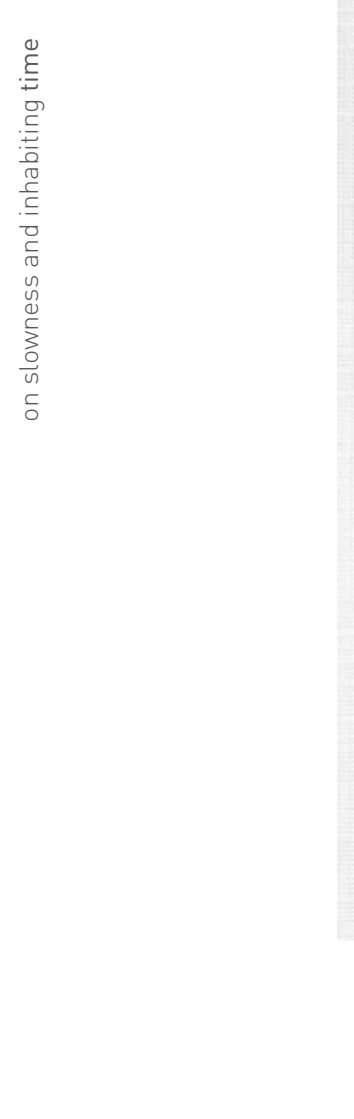



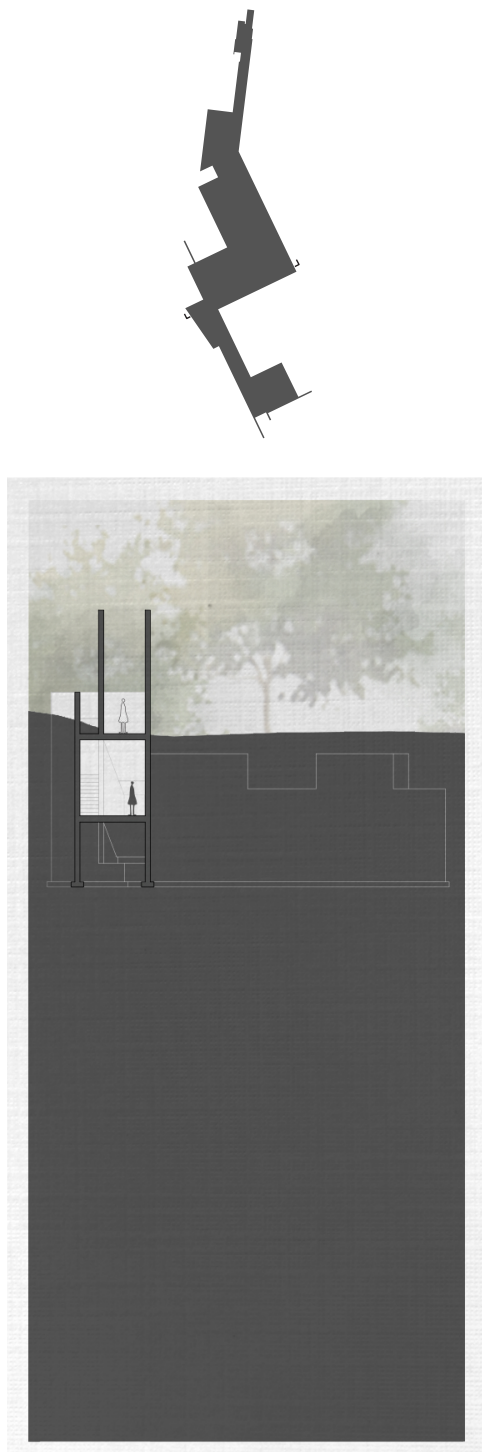

f51

Orthographic Drawings

h_Cross Section Study, Section cc and dd, Sequence 01
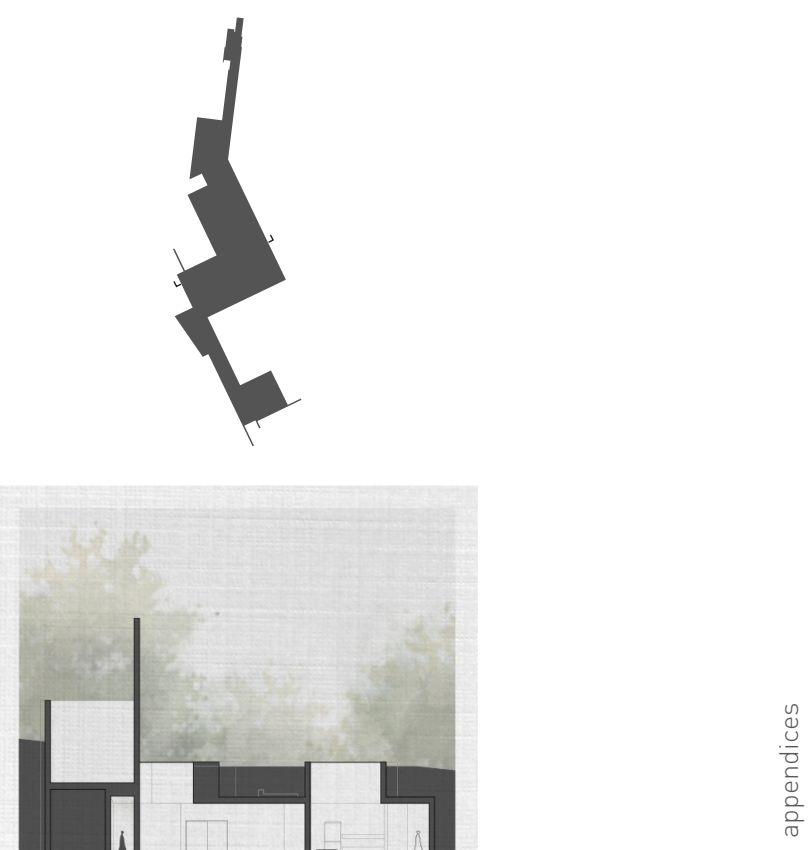

0
0
$\frac{u}{0}$
$\frac{0}{c}$
0
0
$\frac{0}{0}$
$\frac{0}{0}$

(1) $0 \mathrm{~m} \quad 3 \quad 6$ 

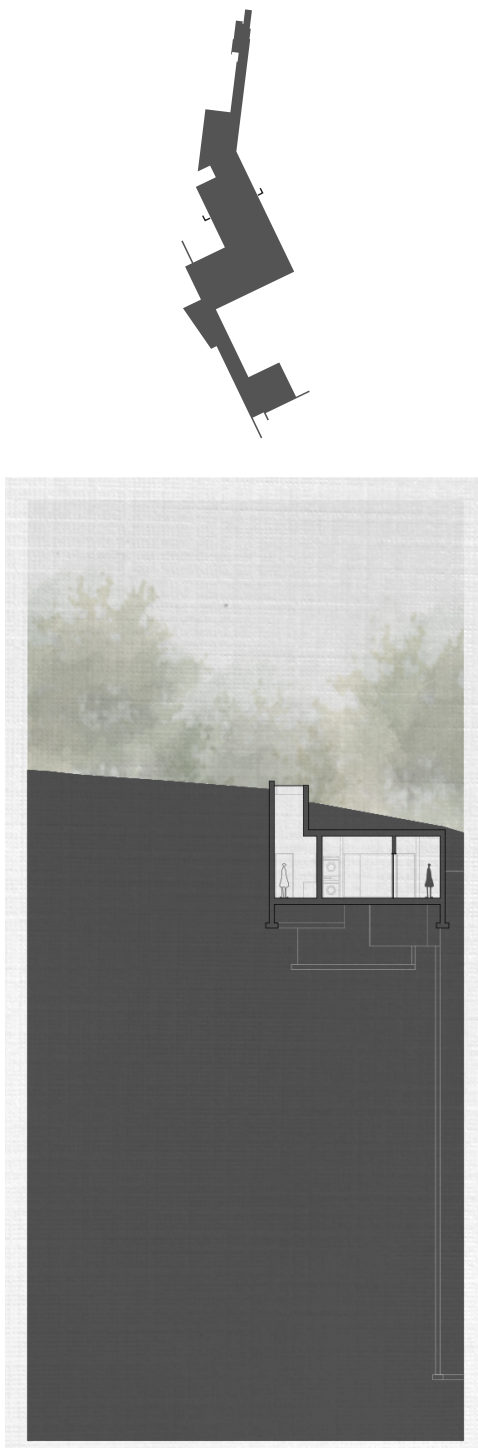

f51

Orthographic Drawings

i_Cross Section Study, Section ee and ff, Sequence 01

51
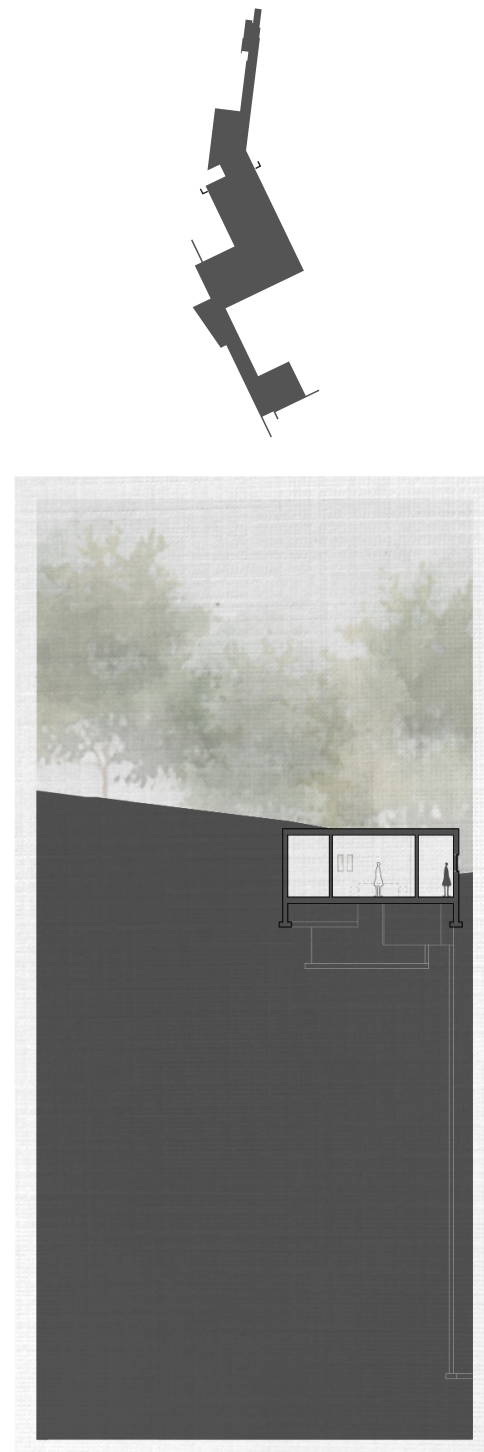

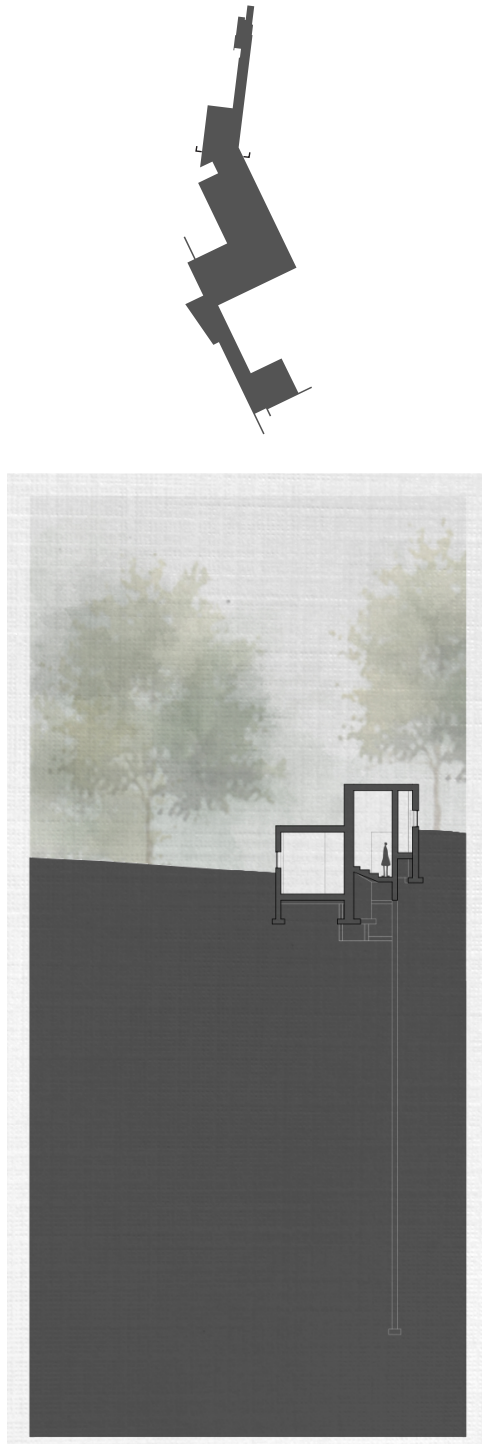

f51

Orthographic Drawings

j_Cross Section Study, Section gg and hh, Sequence 01
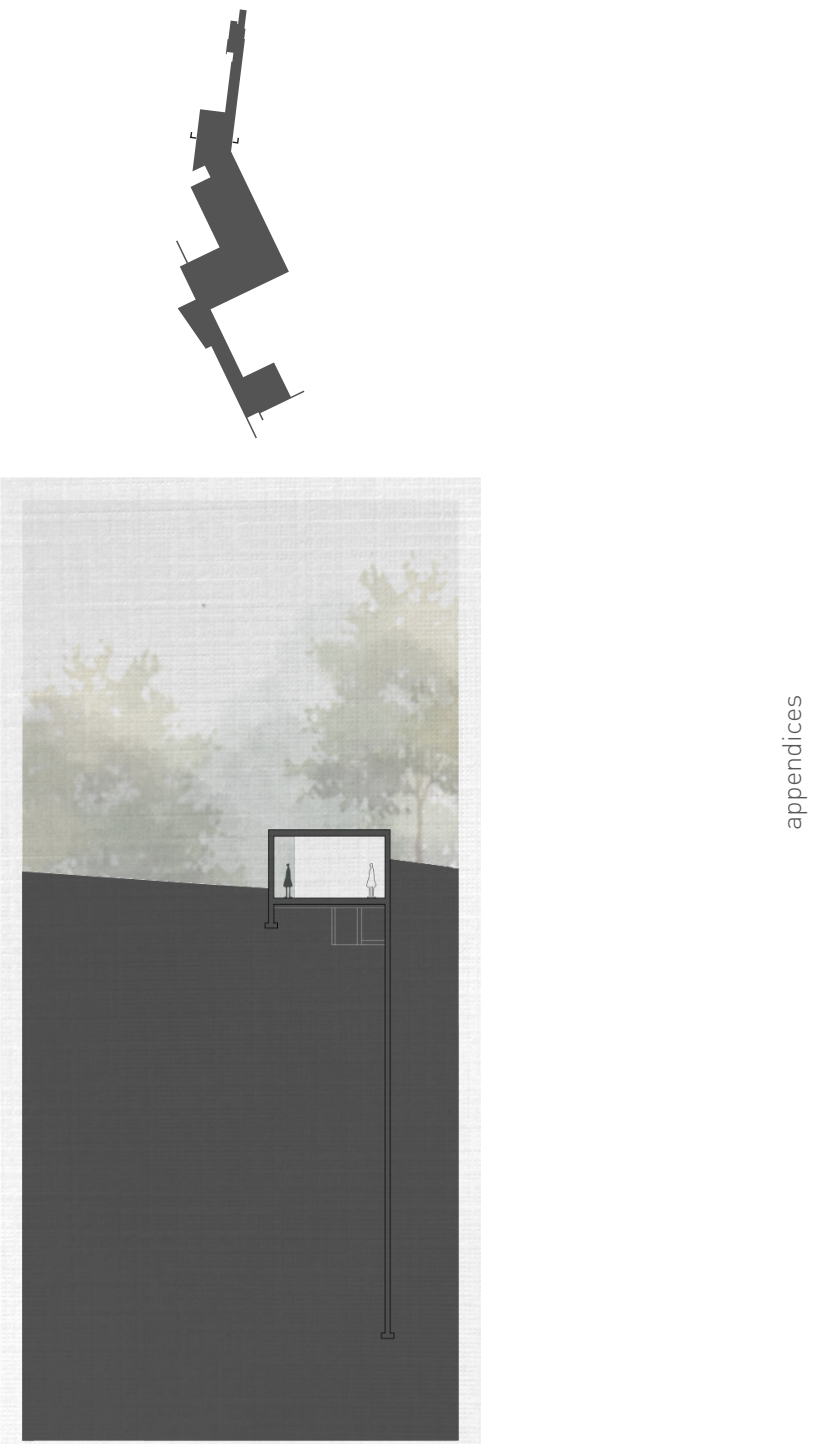

0
0
$\frac{u}{0}$
$\frac{1}{d}$
$\frac{0}{2}$

(1) $0 \mathrm{~m} \quad 3 \quad 6$ 

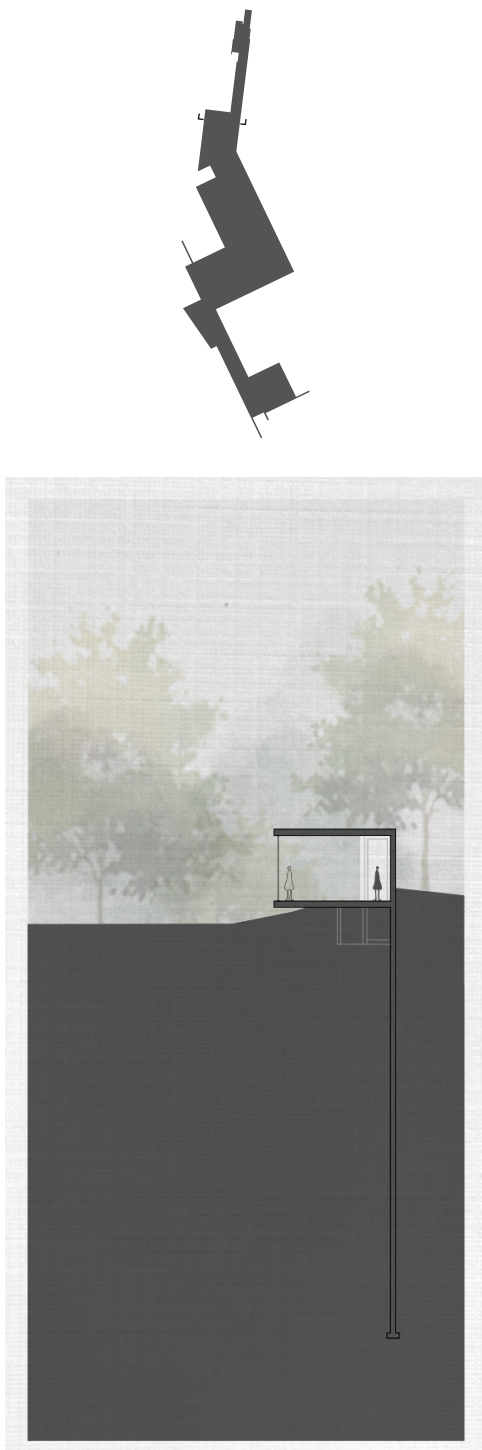

f51

Orthographic Drawings

k_Cross Section Study, Section ii and jj, Sequence 01
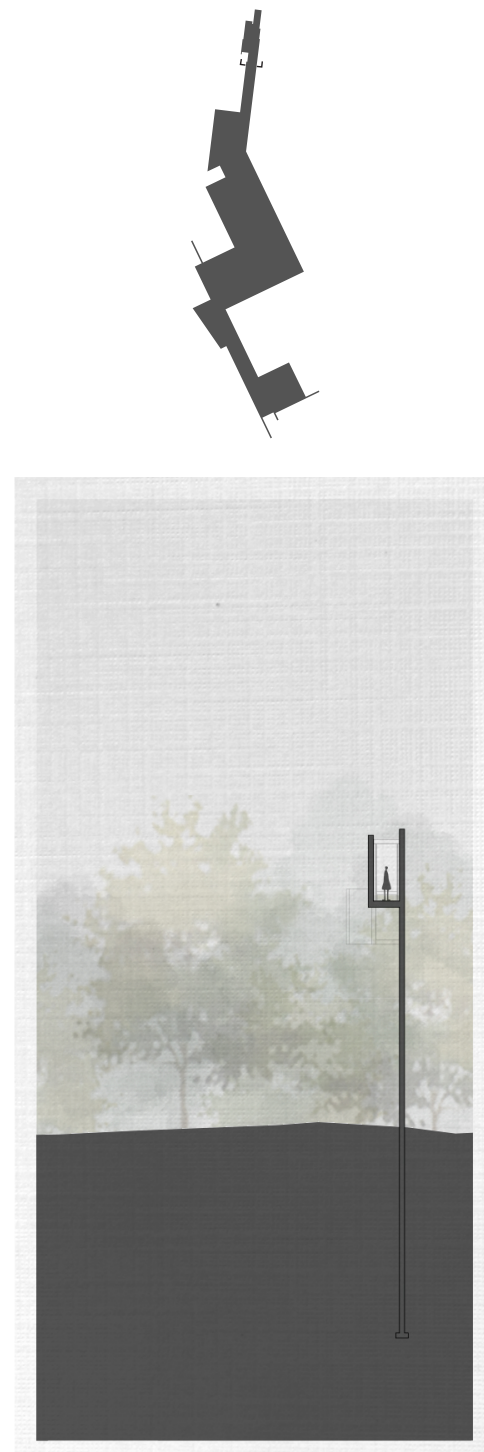$$
01
$$ 

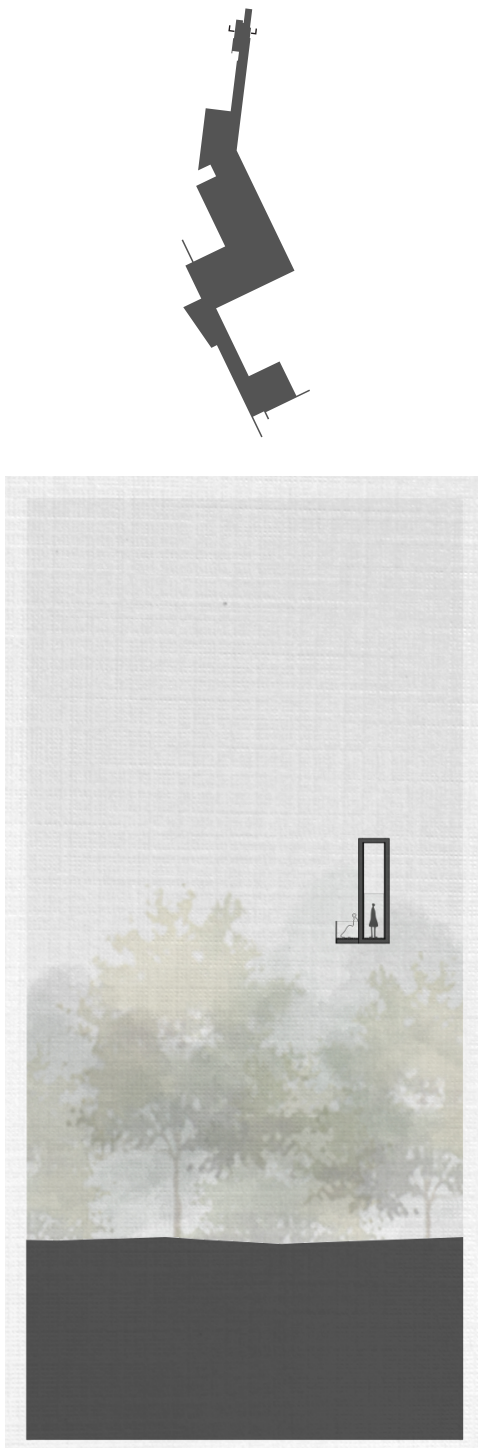

f51

Orthographic Drawings

I_Cross Section Study, Section kk and II, Sequence 01
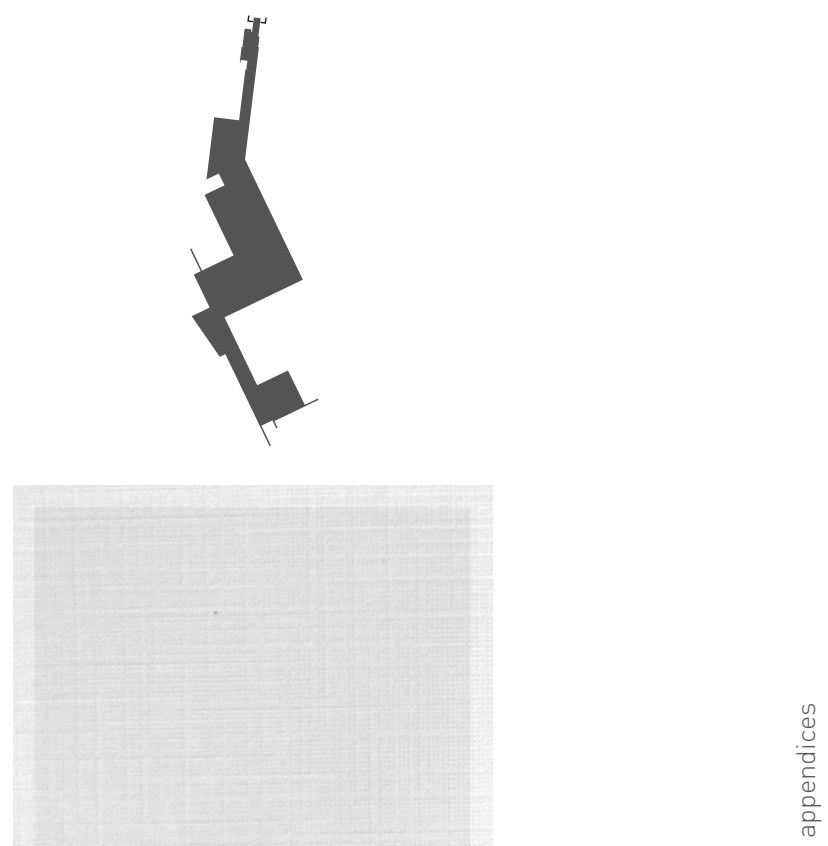

山.

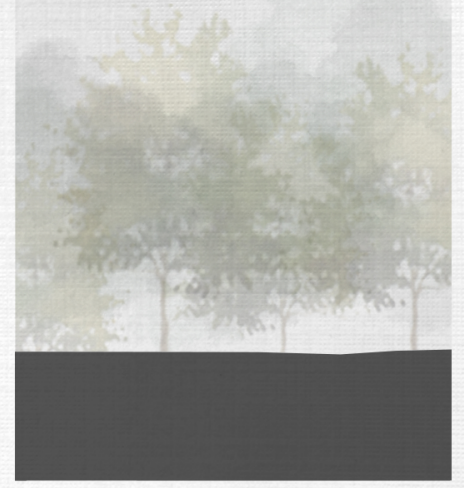

r
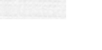

(1) $0 \mathrm{~m} \quad 3 \quad 6$ 


\section{Sequence 02 Appendix C}

The following appendix includes additional drawings and information for Sequence 02 of The House on the Coast. 


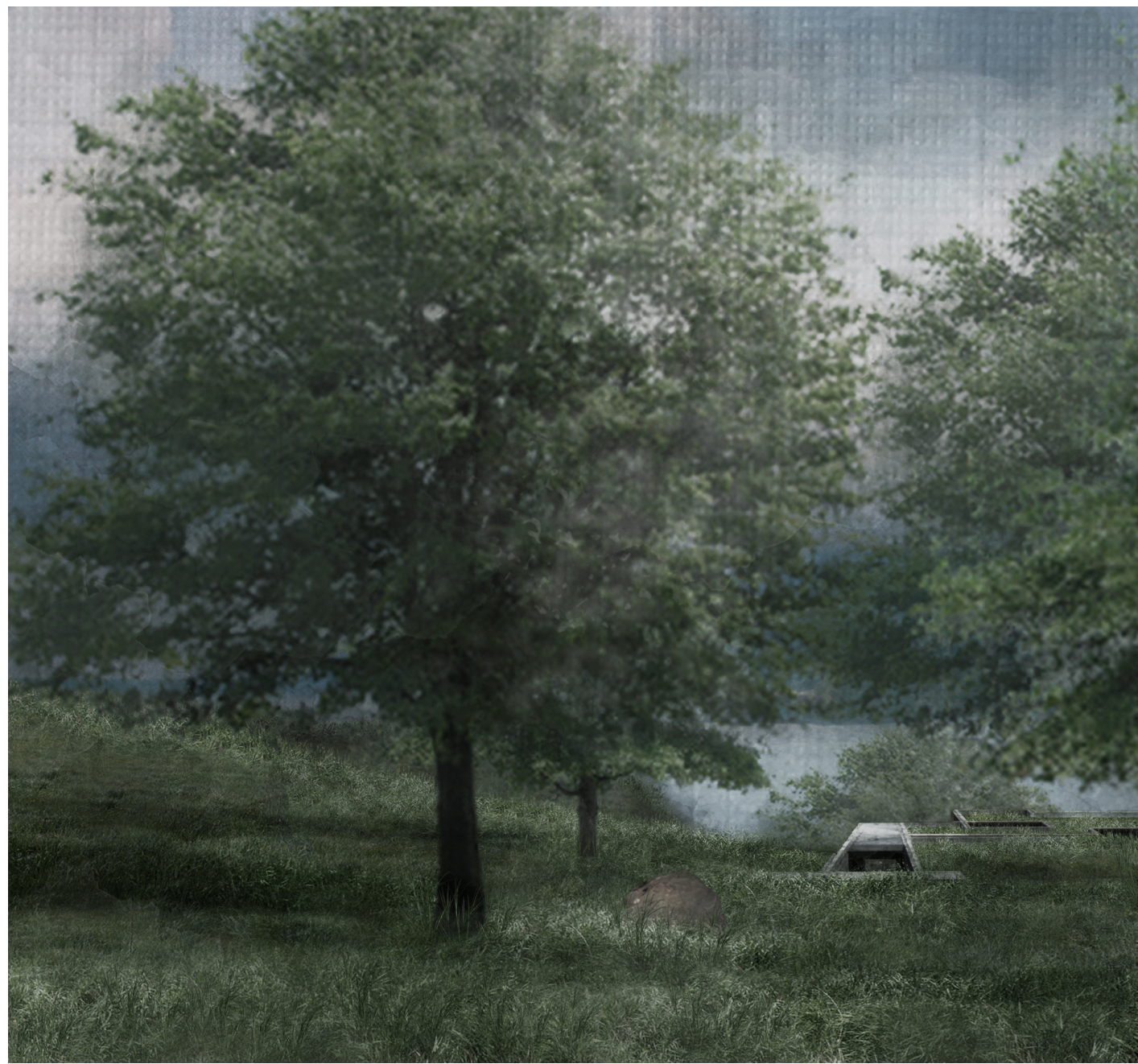

166

Stills: Sequence 02

a_Expansive Site

The expansive view, uninterrupted by the entrance, begins unfolding the architecture and landscape to the inhabitant as they descend towards the entrance of the dwelling. Landscape steps guides one towards two concrete walls that are embedded into the terrain. 


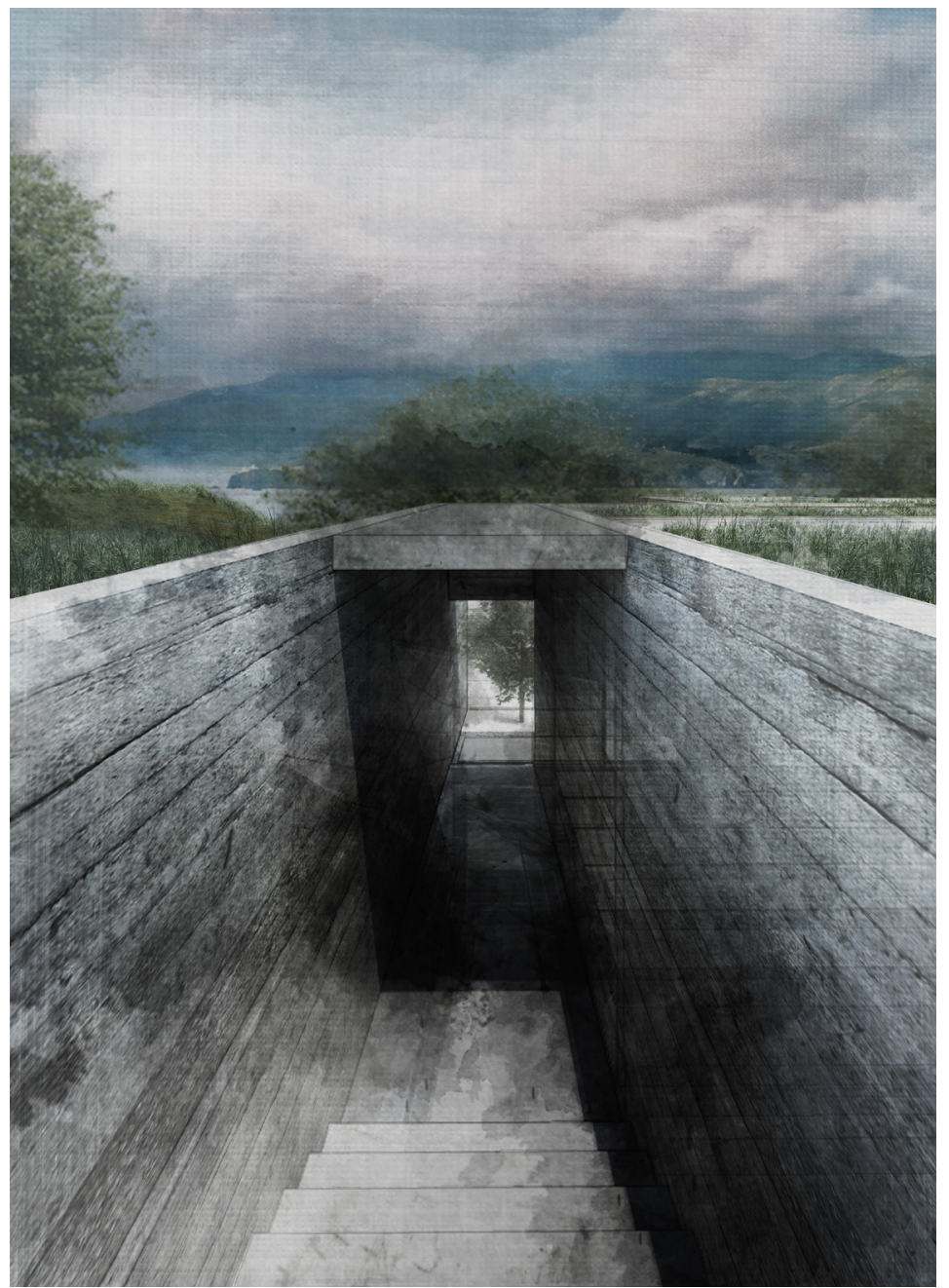

f66

Stills: Sequence 02

b_Entrance Steps

On one side, an angled wall extends time by manipulating one's perception into believing the procession is longer than it actually is. Cut views of the Pacific Ocean, the rock garden in the distance, the sky and the surrounding concrete walls, conceal and selectively reveal elements of both the architecture and the landscape to the inhabitant as they traverse towards the entrance. 


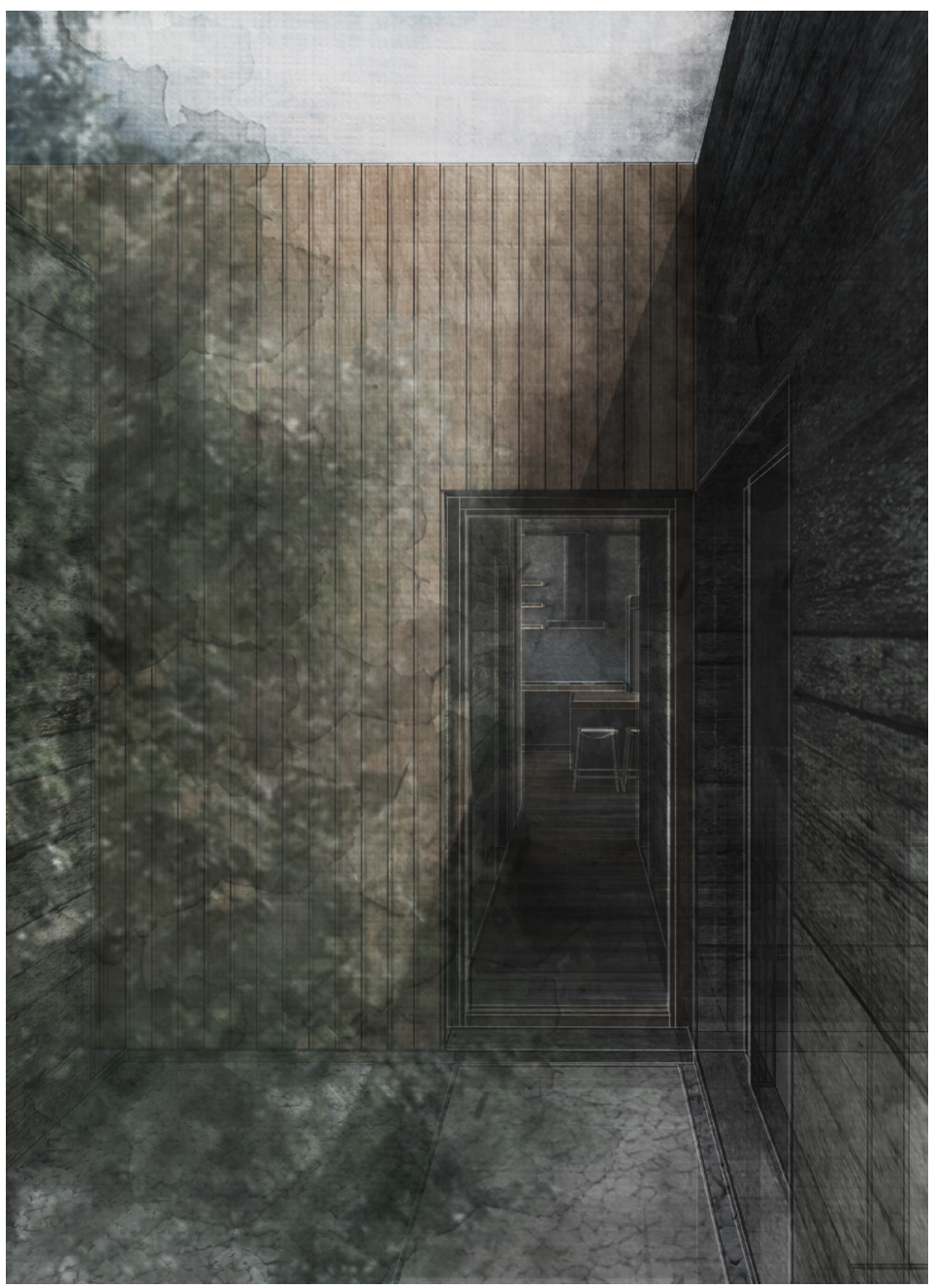

f66

Stills: Sequence 02

c_Rock Garden

As the inhabitant arrives at the rock garden and turns towards the doorway, a cut view through the shortcut expands to reveal the kitchen in the distance. This expansive cut exposes the approaching space, and yet, moves the inhabitant through a large concrete door and into darkness before revealing itself entirely. 


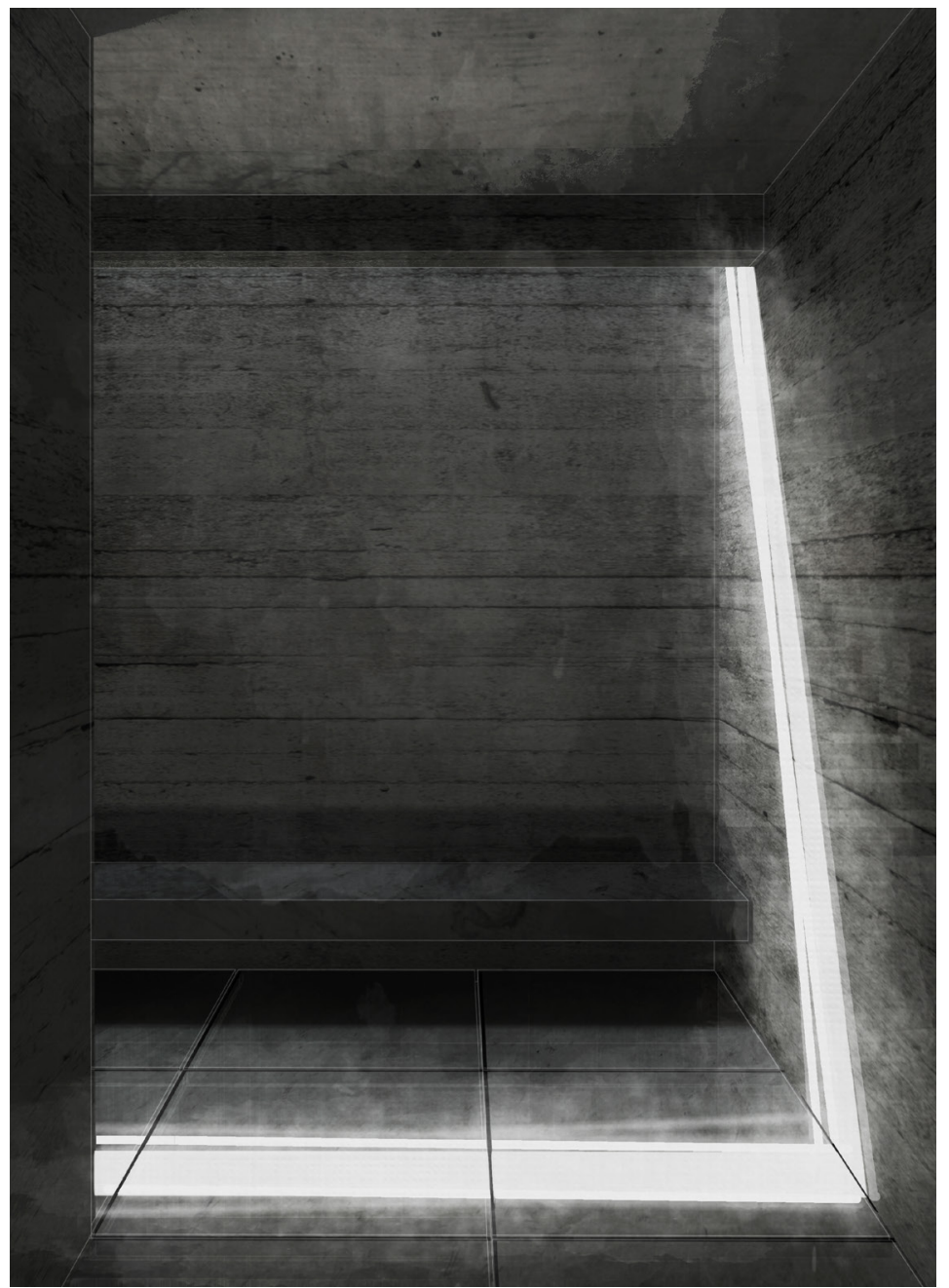

f66

Stills: Sequence 02

d_Foyer

By revealing and simultaneous concealing the approaching slowed space, a nonlinear passage directs the inhabitant through a dark foyer that is guided by a slit skylight that illuminates the bench and the concrete wall ahead. This transition was maintained from the first sequence, as its contrasting qualities further heightens the expansive space to come. 


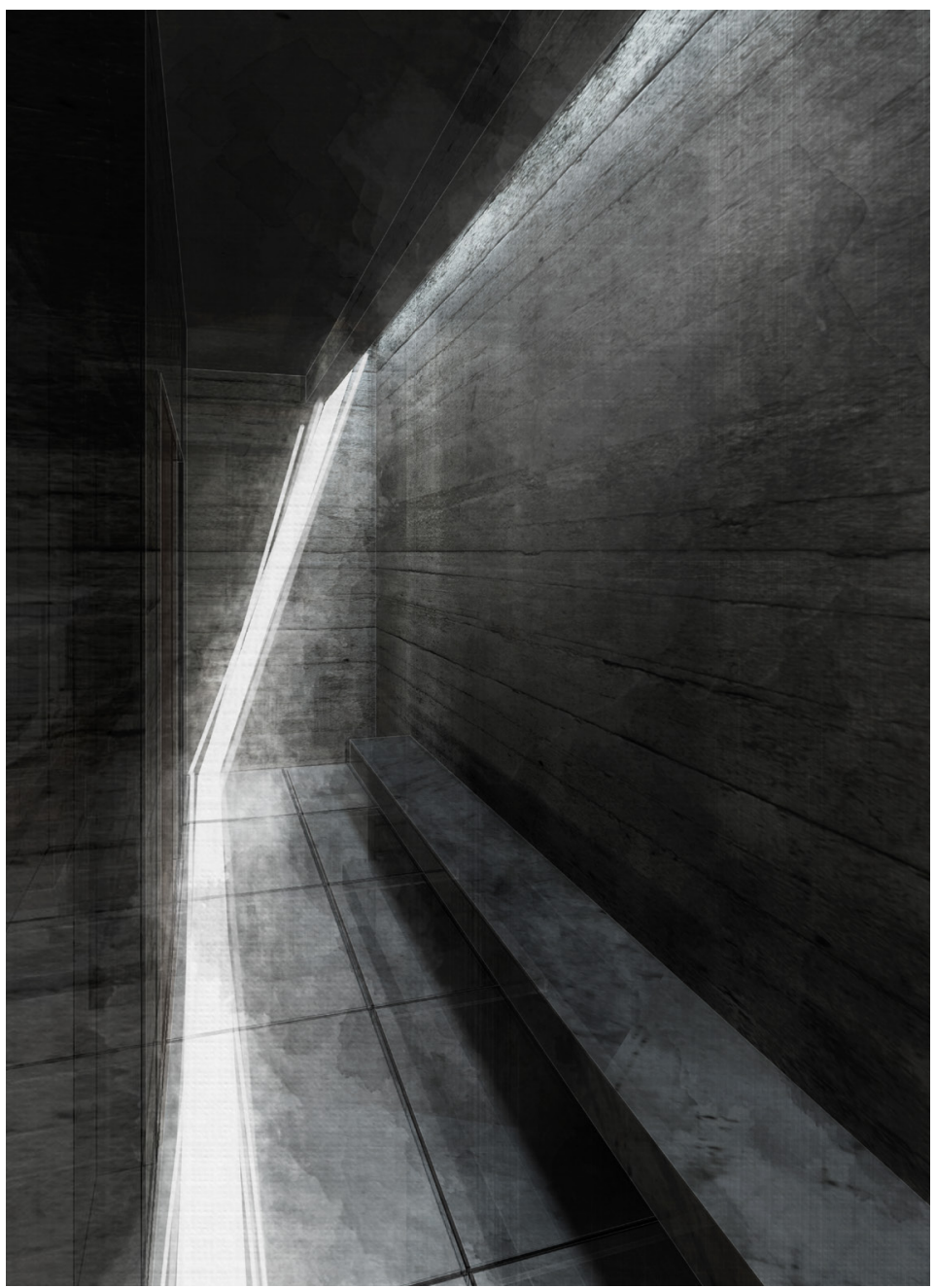

f66

Stills: Sequence 02

e_Foyer Bench

At the end of the corridor, one turns to find themselves situated between two compressing walls; the slit skylight pours light onto the floors and walls of the foyer. The light guides the inhabitant through the foyer procession towards the expansive kitchen and dining space. 


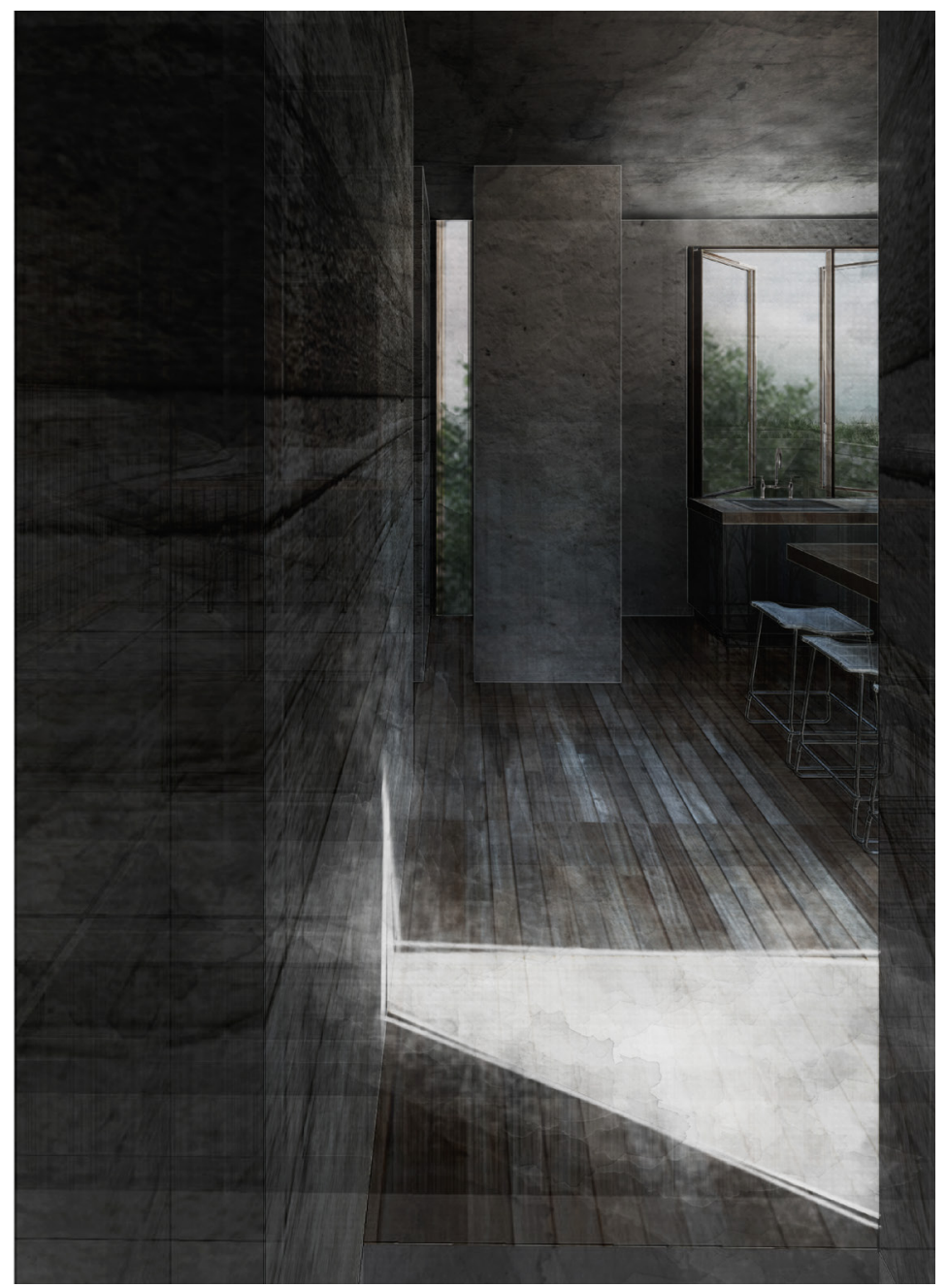

f66

Stills: Sequence 02

f_Cut Reveal

A single slit window cuts to reveal the garden below; with this procession the entrance slits are experienced in the focus range of one's view, whereas, the alternate procession, through the shortcut, reveals the slits in one's peripherals. Although the alternate is not dimly lit, the increased speed with peripheral cuts creates a similar experience between the two. 


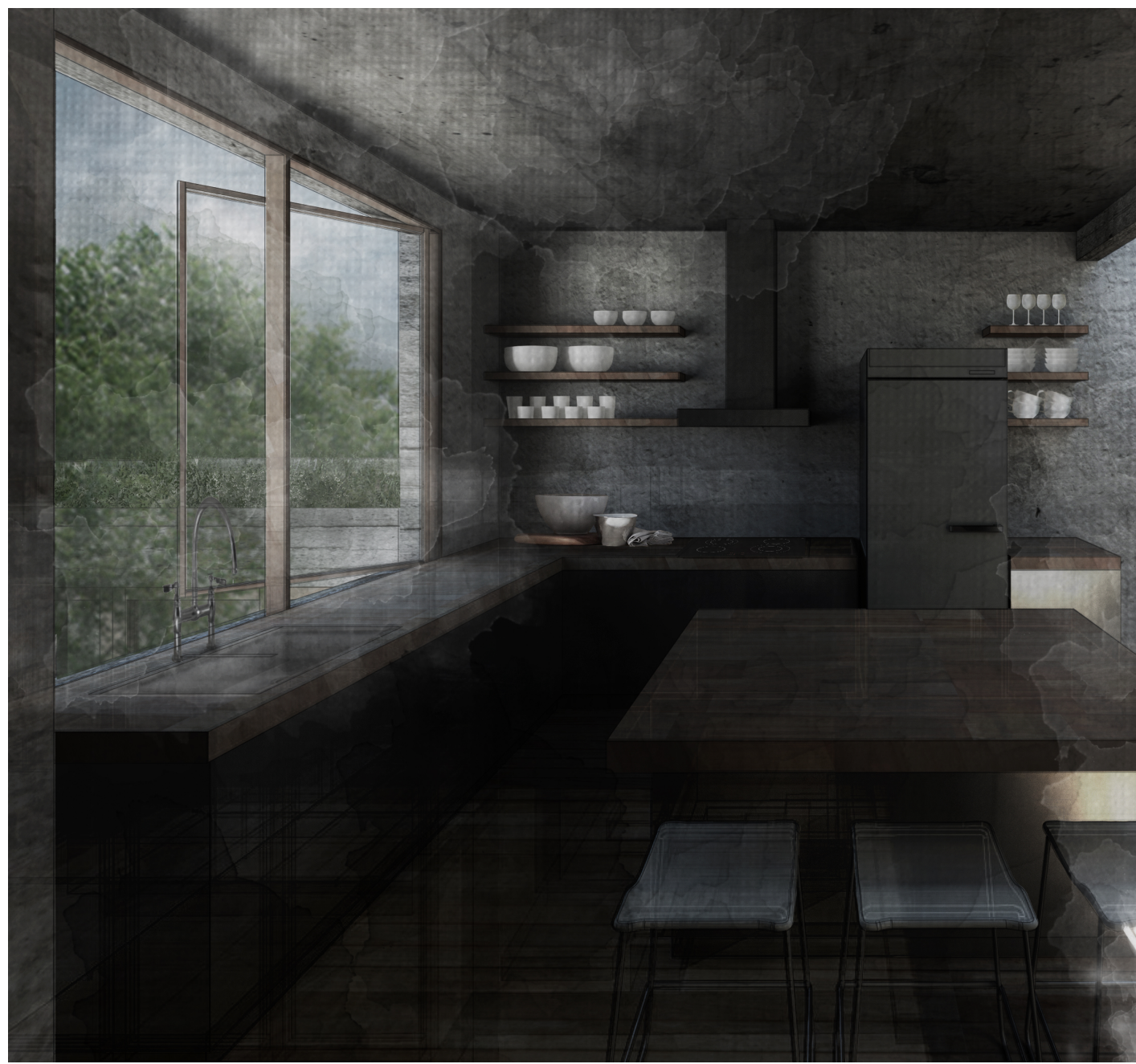

f66

Stills: Sequence 02

g_Moment of Pause; Kitchen and Dining Room

As the inhabitant moves towards the large casement window, they are turned and situated in the slowed space of the kitchen and dining room. The central axis, which is located from the window to the large skylight above the dining room table, overlaps interior and exterior spaces while juxtaposing with the dimly lit foyer to create a moment of pause within the procession. 


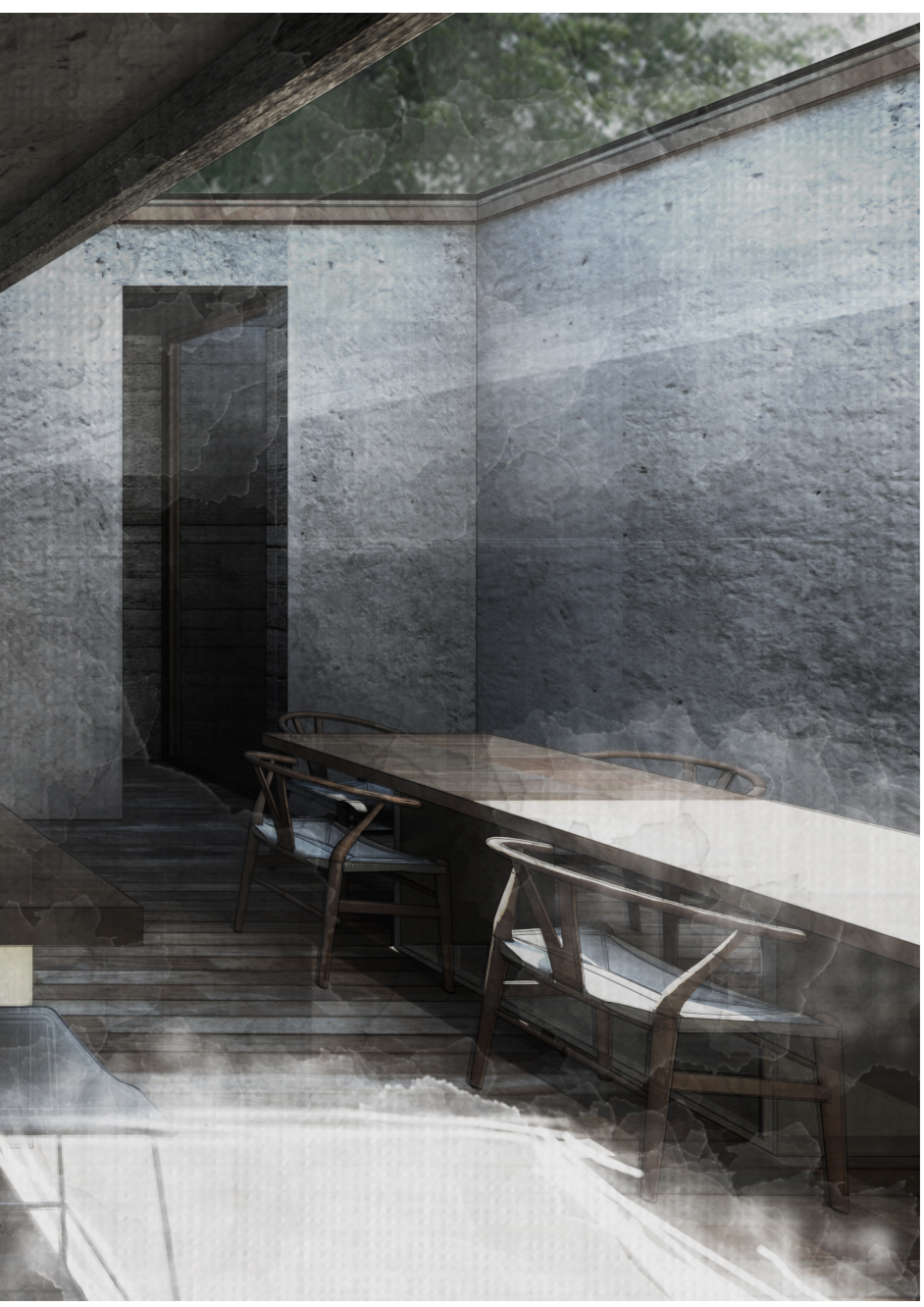

As one walks towards the skylight, the heaviness of the walls, and lightness of the sky, consumes the inhabitant. They look up towards the sky and directly through to the garden below and Pacific in the distance. The space becomes a gap, an emptiness that allows one to be still in a specific space or moment; losing track of time but also being very aware of its passage. 


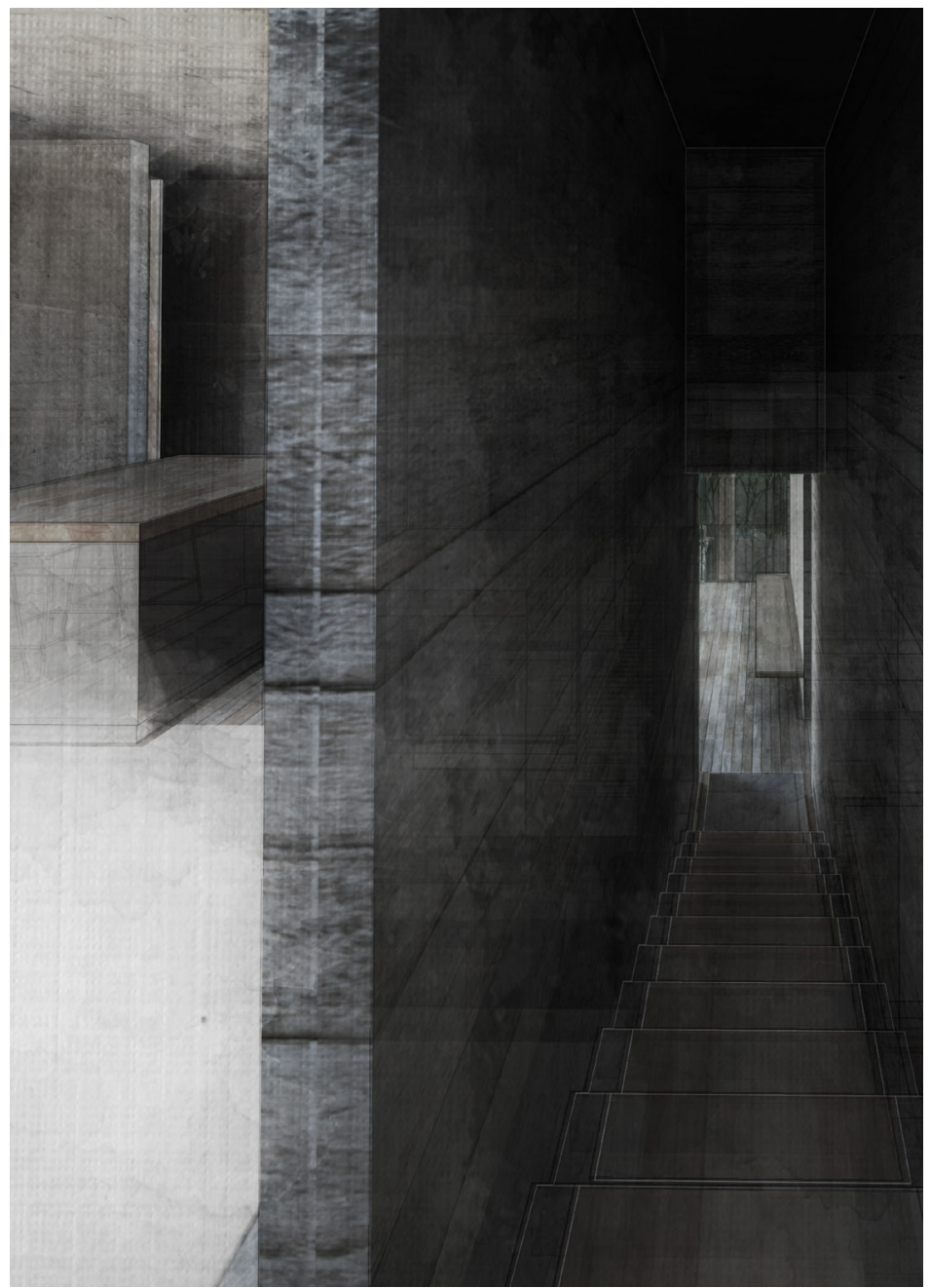

f66

Stills: Sequence 02

h_Descent To Garden

Turning the corner situates the inhabitant between two walls which compress towards a seemingly narrow corridor. The stairs, concrete with wooden inserts, juxtapose the floor while referencing it, and yet, the final step is disconnected from the staircase and made of concrete. This irregularity within the rhythm of the procession creates a distinct moment that presences the mind and body. 


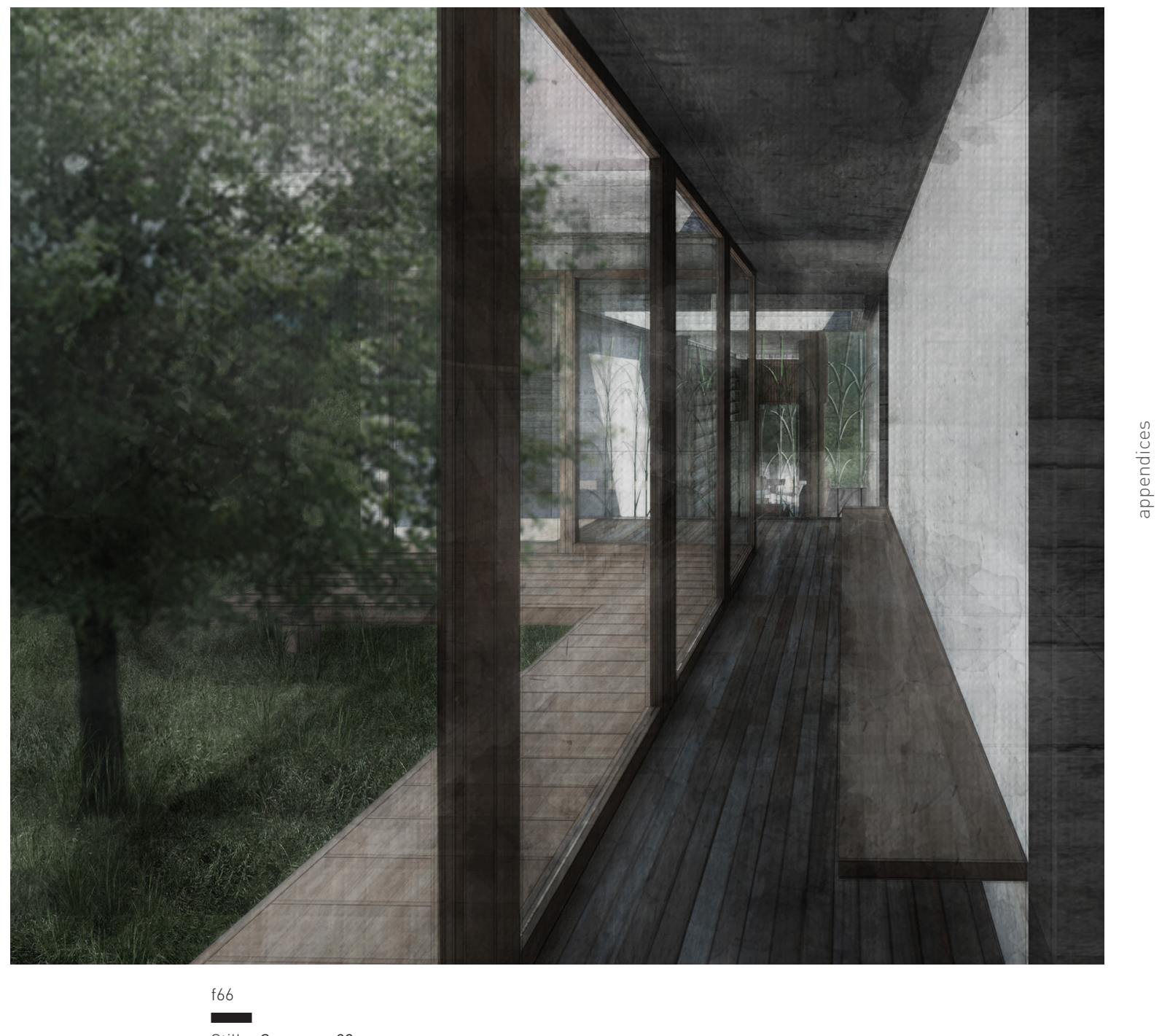

Stills: Sequence 02

i_Moment of Pause; Garden

At the bottom of the stairs, one's peripherals are situated within the expansive East-West axis between the bench and garden, while focus is placed on the NorthSouth axis between the first floor lightwell and cut views behind the bamboo wall ahead. The glass walls of the garden open to allow the elements, the wind, rain, and sun, to penetrate the interior. 


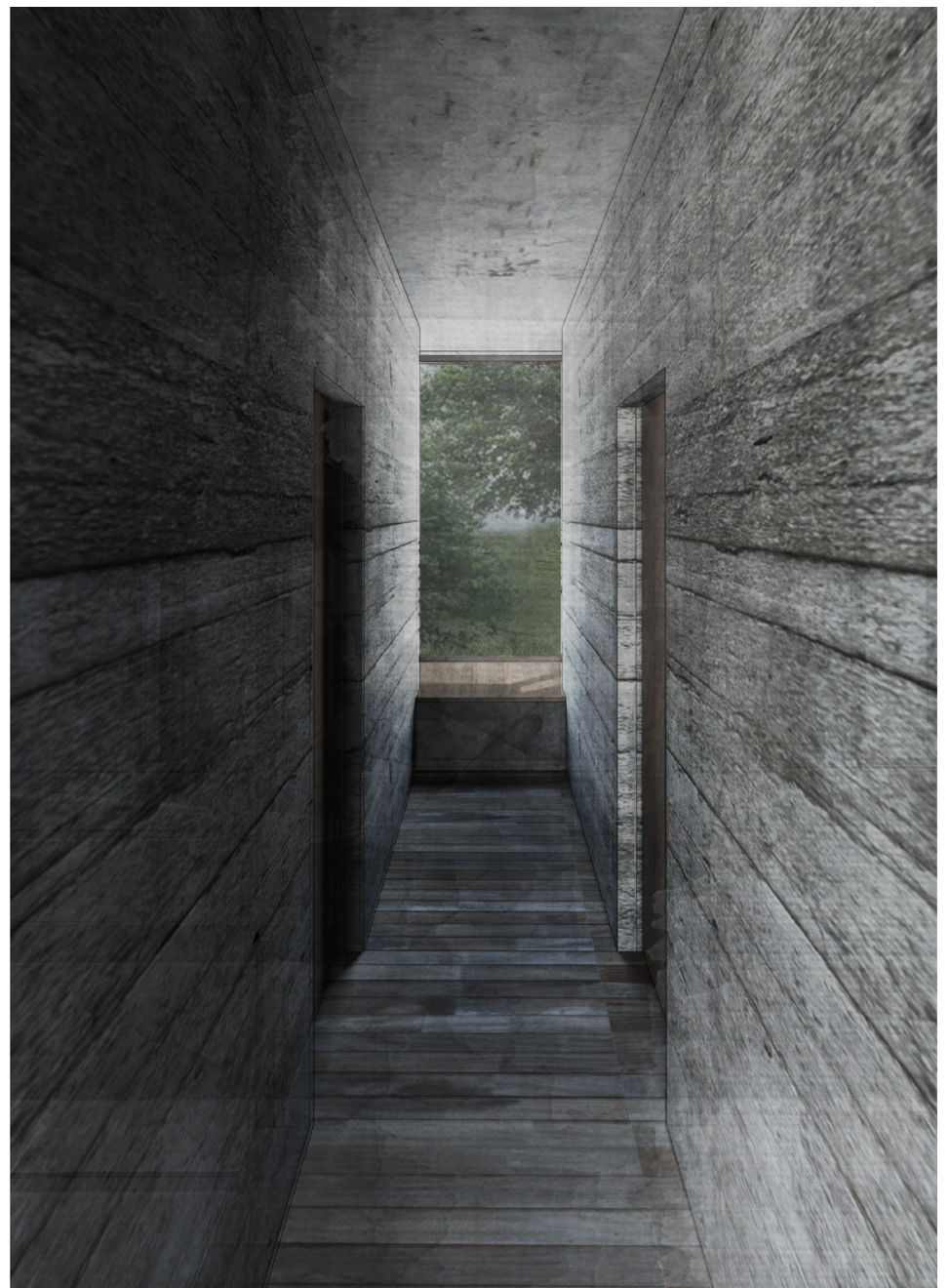

f66

Stills: Sequence 02

j_Bench

The inhabitant turns to situate themselves between two walls, looking towards a window seat. The window frame disappears as the view extends past the adjacent walls, and yet, as the inhabitant walks towards the seat, it begins to push both out and into the space while expanding the view of the terrain; in turn, allowing one to sit both outside and inside at the same time. 


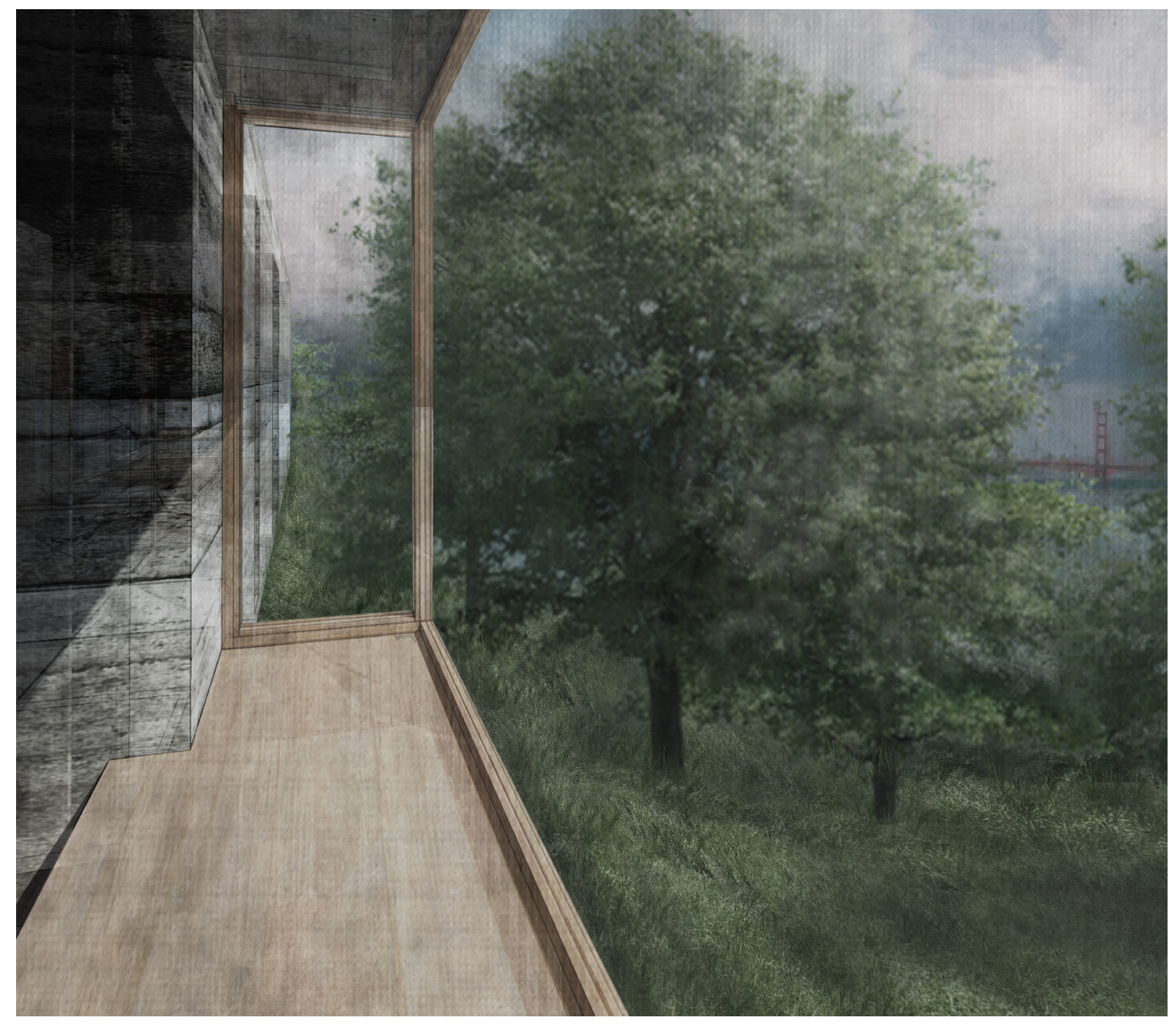

f66

Stills: Sequence 02

k_Moment of Pause; Bench

While resting on the bench, the view expands further as glimpses of the Golden Gate Bridge reveal themselves through the trees. The sound of the ocean intensifies as the window opens to allow the atmosphere and elements of the coast to penetrate within. This space of solitude, shifts back, allowing the inhabitant to nestle and hide away into a place all their own. 


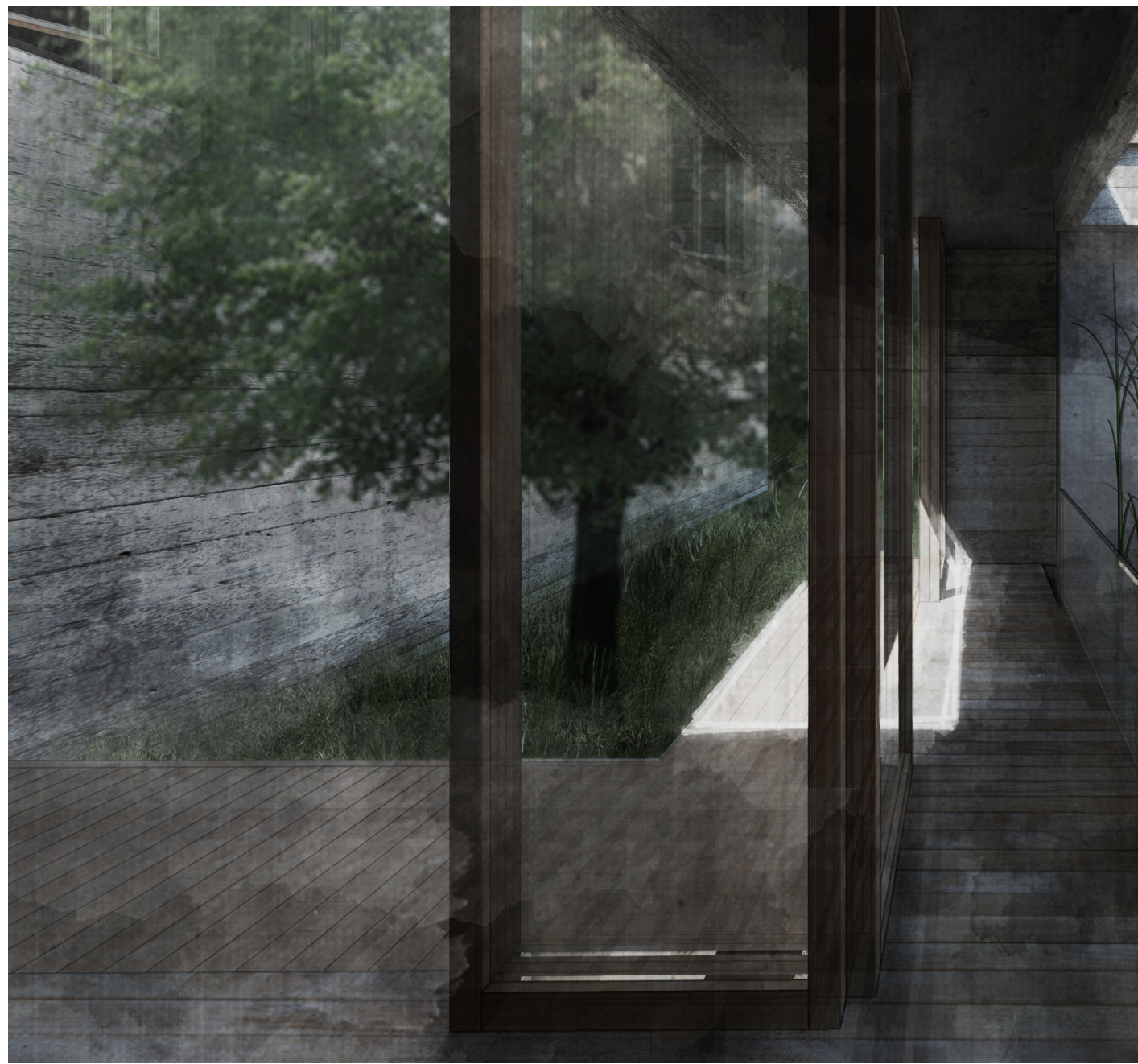

f66

Stills: Sequence 02

I_Moment of Pause; Overlapped Garden and Living Space

Afterwards, the inhabitant follows the narrow corridor and turns to walk towards the bamboo wall. The rhythm of the wall cuts the expansive view by creating distinct perceptual slices in space and time. One stands, overlooking the living space below and garden to the left. This overlapping of spatial and temporal moments references the tumbling of the wire model while creating stillness. 


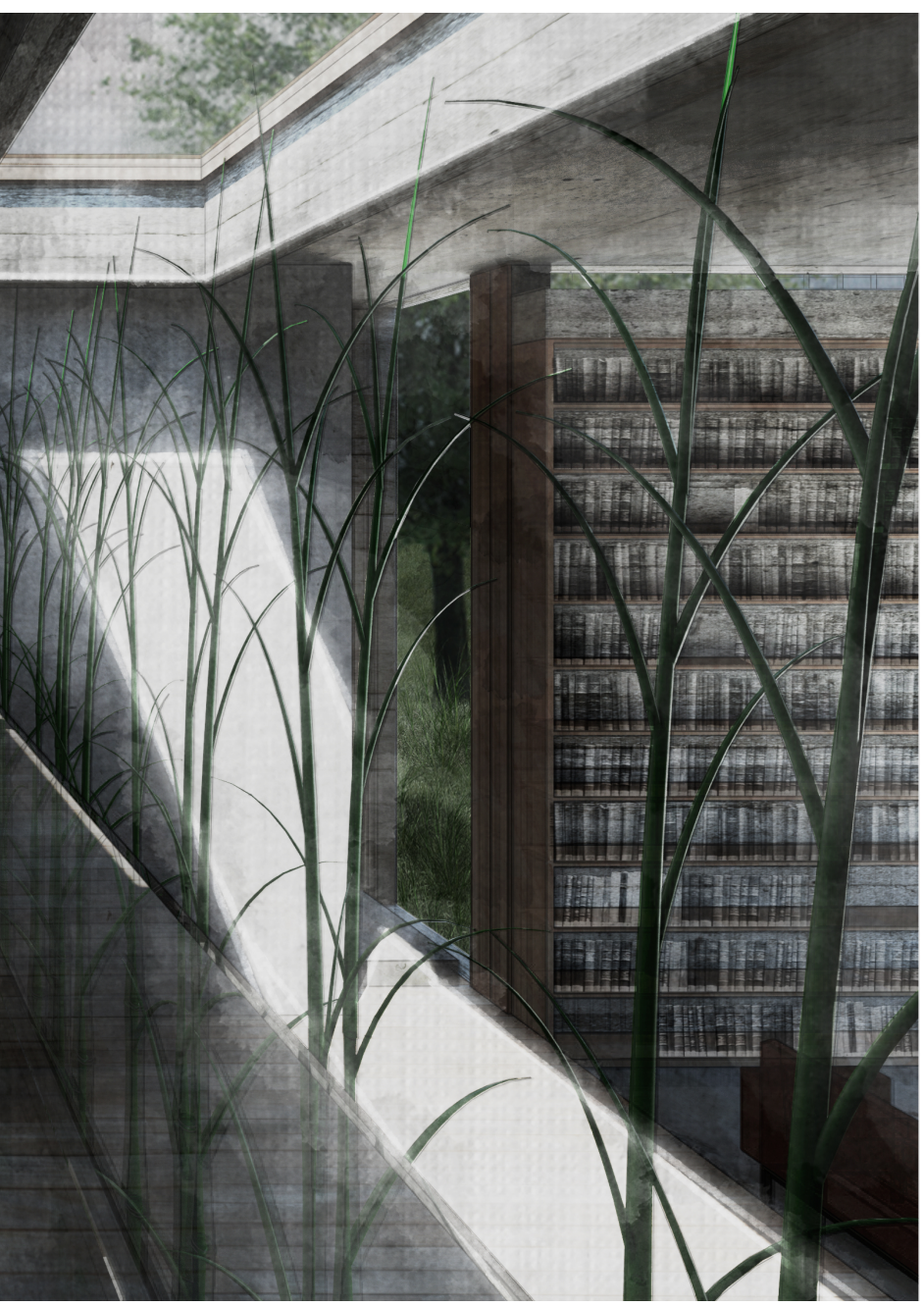

This stillness results in the inhabitant's mindful physical presence of the overlapping of space and time with their mind and body. In this sense, expansive views harness sustained attention and slowness by moving the eyes and body slowly through a sequence of architectural frames. 


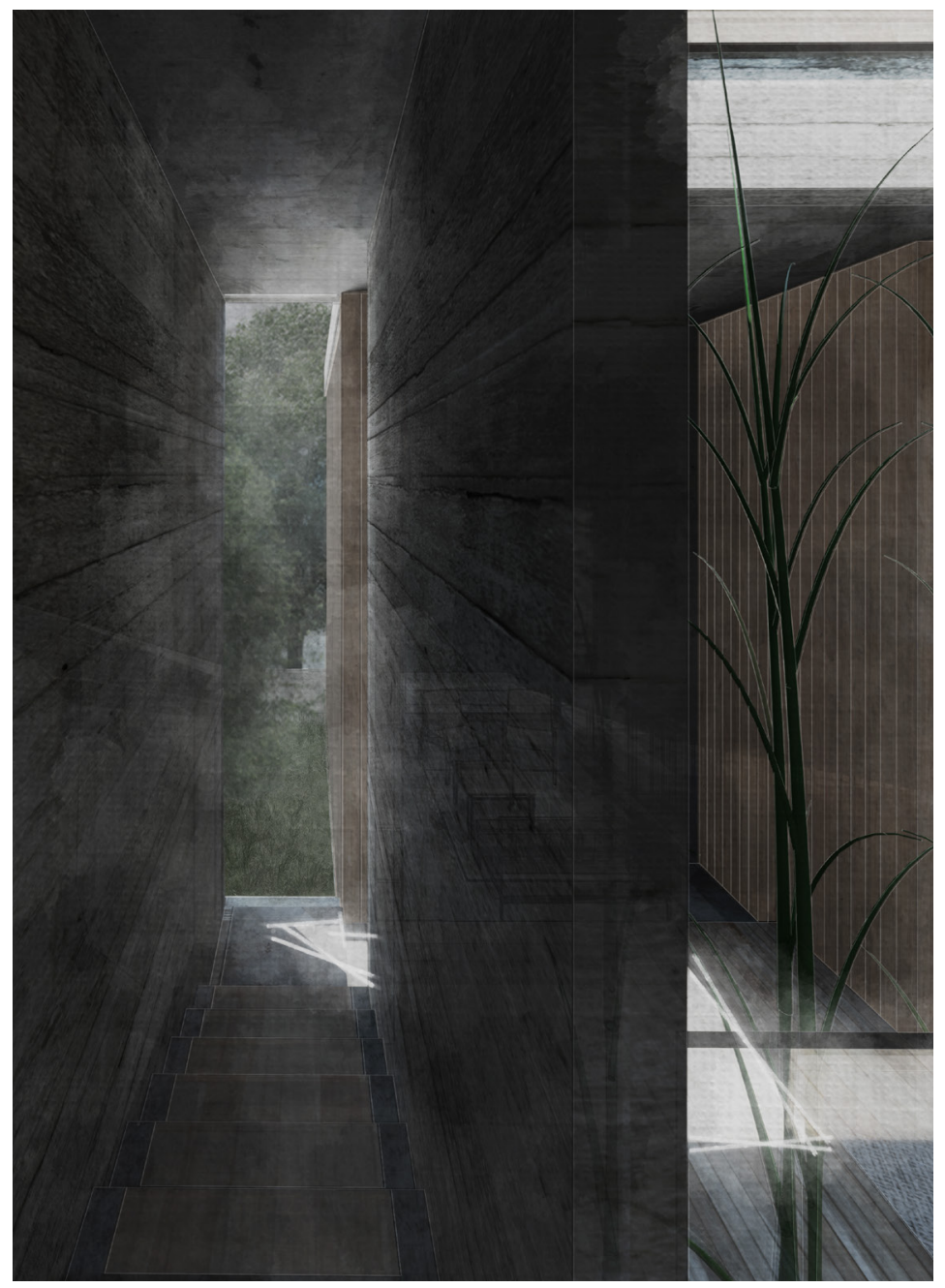

f66

Stills: Sequence 02

m_Descent to Living Space

As the inhabitant walks past the bamboo wall, their peripherals cut rapidly, speeding up the procession towards the final stairs, situated between two walls, and cuts to a view of the landscape. The compressed stair heightens suspense and anticipation by momentarily concealing the final slowed space before revealing it again. 


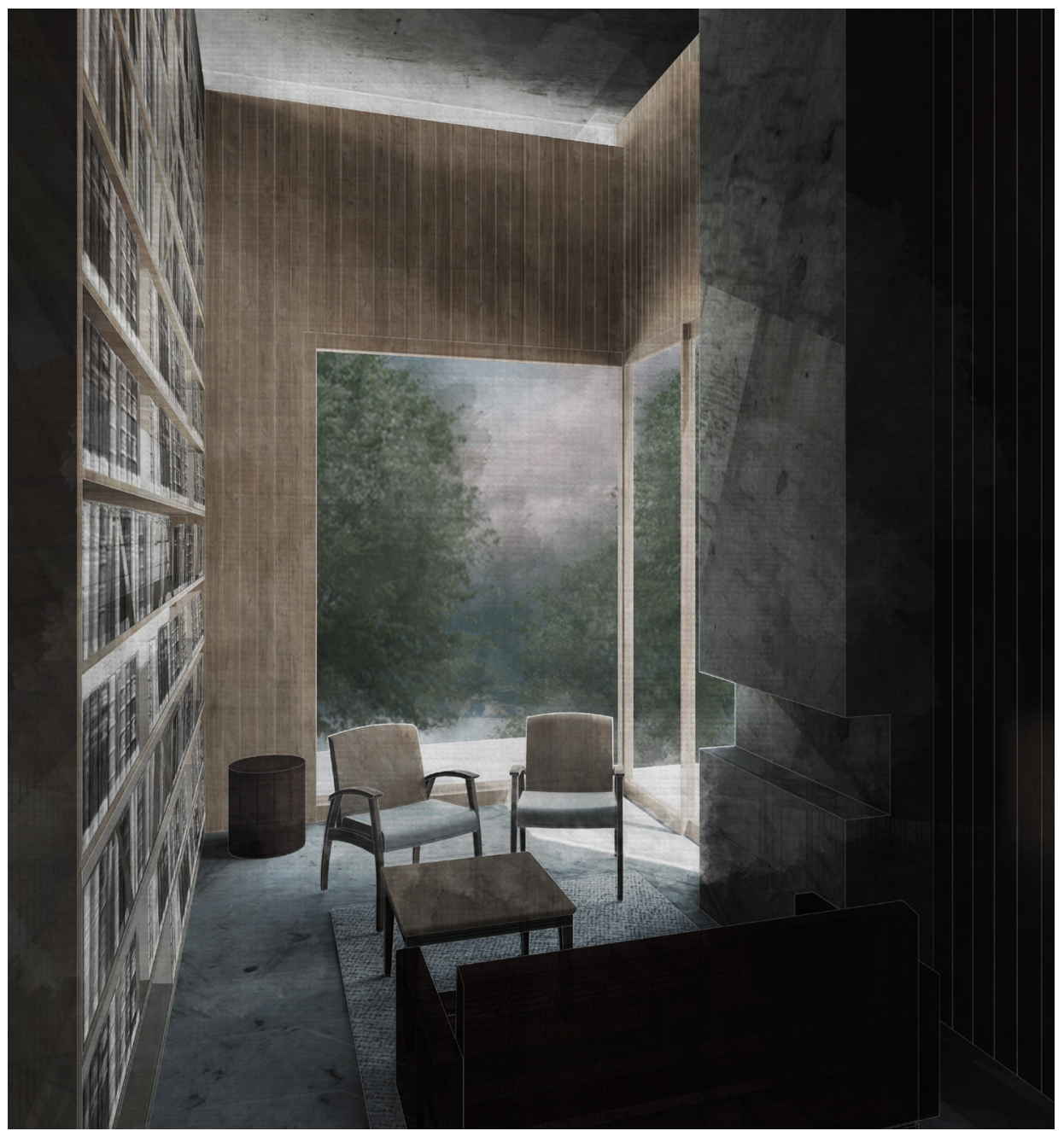

f66

Stills: Sequence 02

n_Moment of Pause; Living Space

The clerestory window above is unseen, light penetrates the space while the corner window opens up to a patio set on top of the descending landscape. As the inhabitant moves towards the exterior and finally out onto the patio, landscape steps begin the descent towards the ocean while the sound of water crashing against the rocks intensifies as a storm slowly rolls in. 


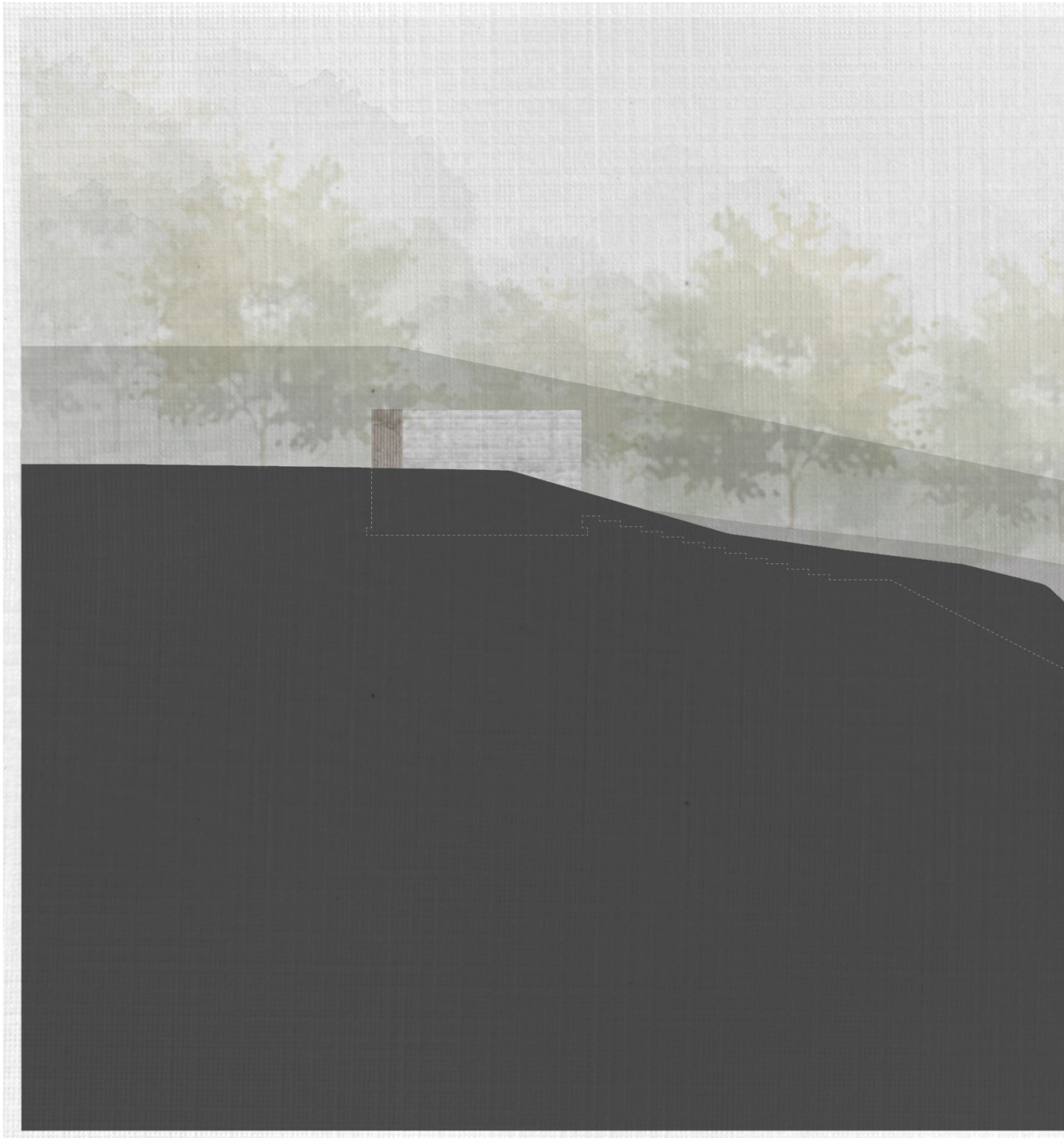

Orthographic Drawings

m_East Elevation, Sequence 02 
(1) $0 \mathrm{~m} 3 \quad 6$ 


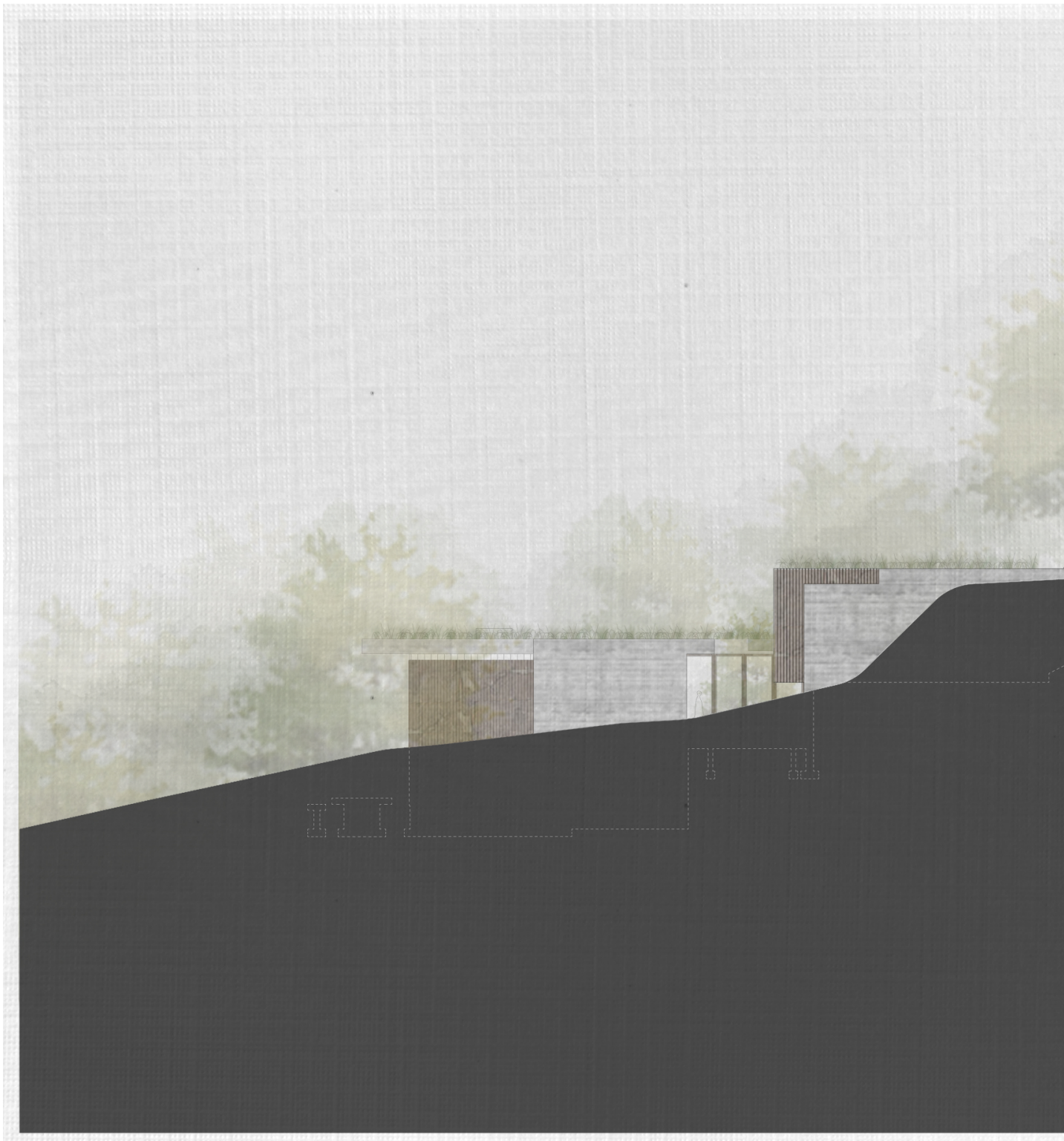

f57

Orthographic Drawings

n_West Elevation, Sequence 02 
(1) $0 \mathrm{~m} \quad 3 \quad 6$ 


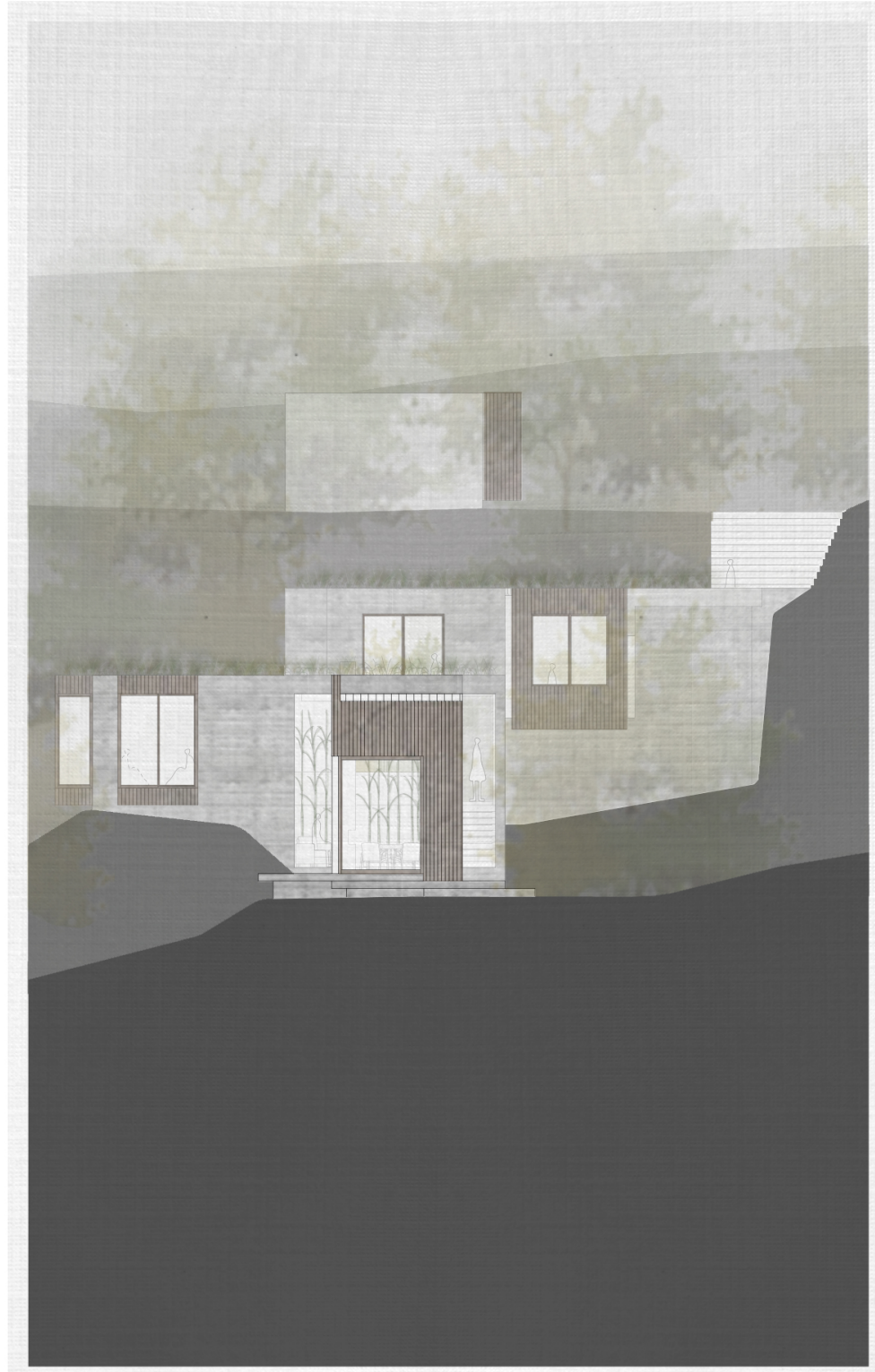

\section{Orthographic Drawings}

o_North Elevation, Sequence 02 
(1) $0 \mathrm{~m} \quad 3 \quad 6$ 


\section{Temporal Representation Appendix D}

The following appendix includes design research models used towards temporal tangibility and the conception of both Sequence 01 and 02 of The House on the Coast. 


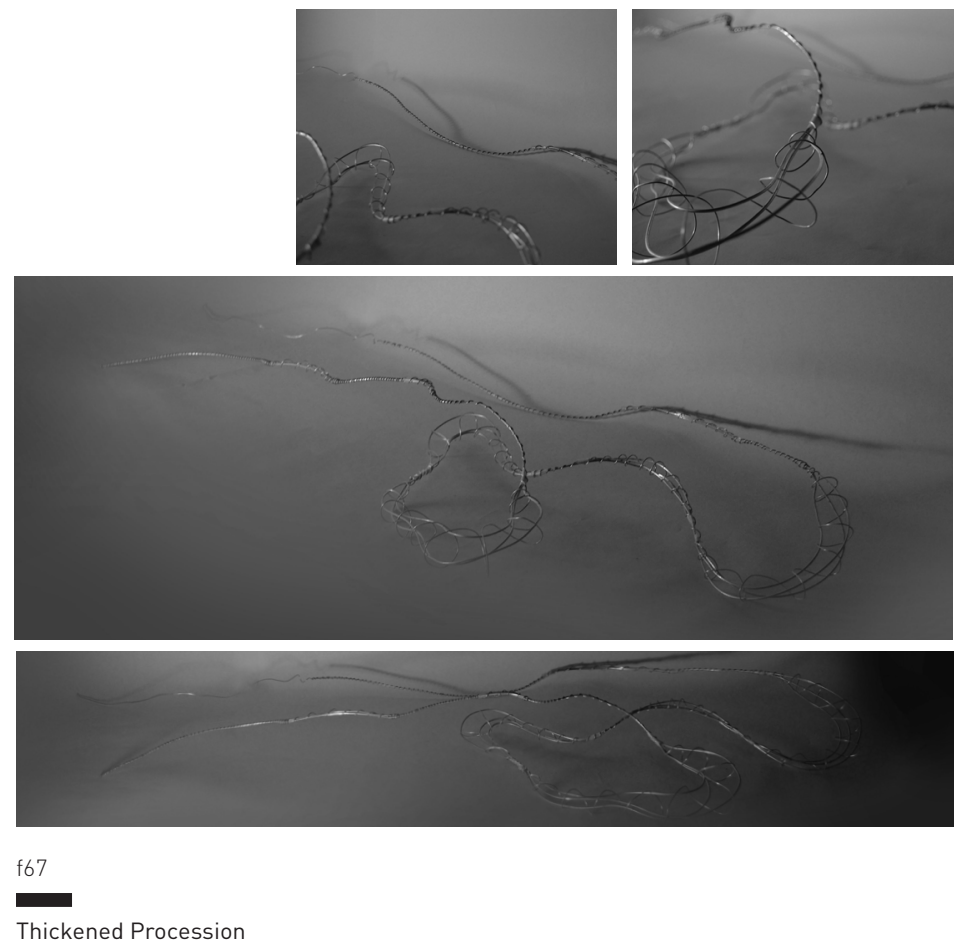



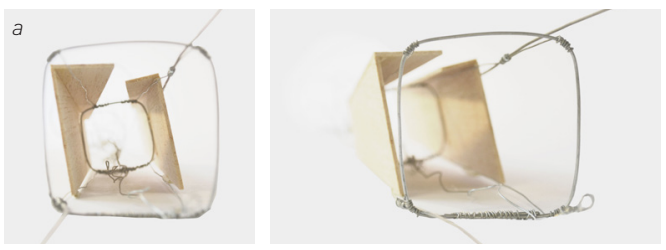

$b$
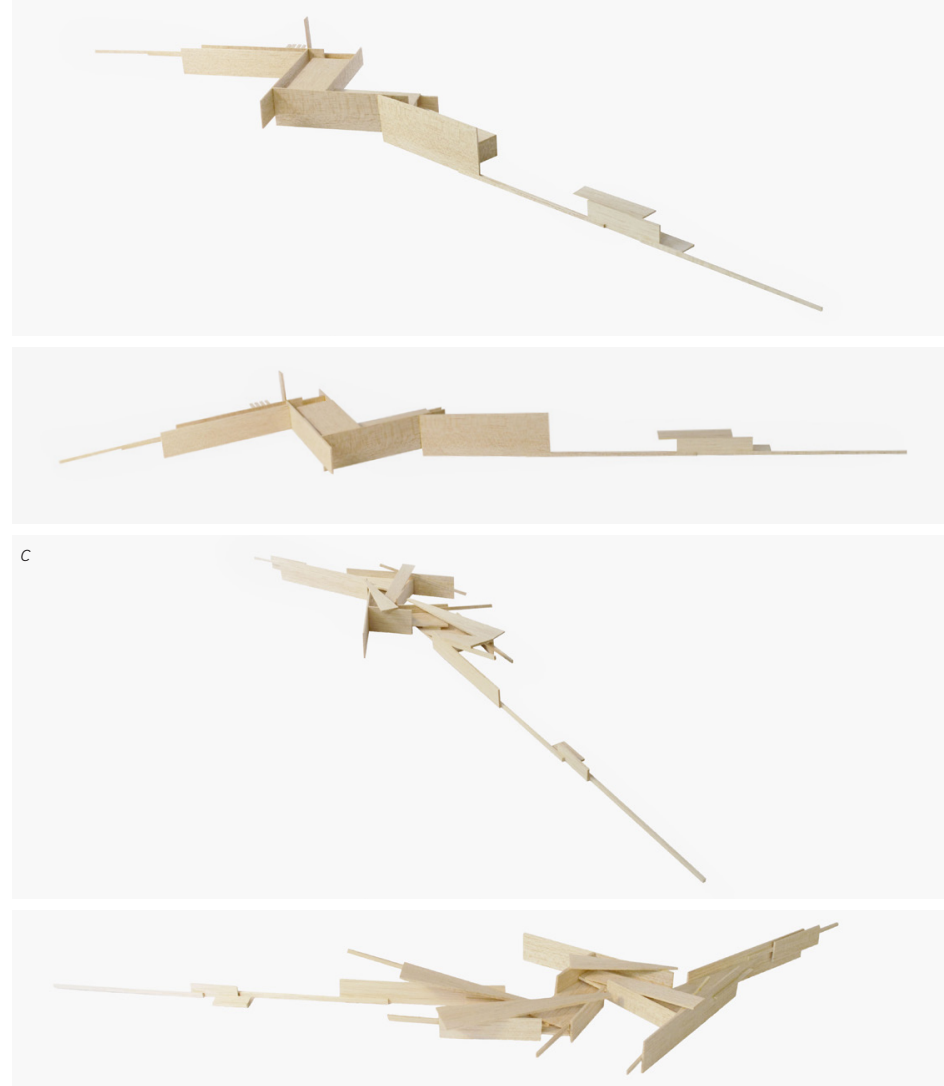

f68

Conceptual Models

a_Spatial and Planar Collision; b_Orchestrating Silence; c_Orchestrating Chaotic Silence 

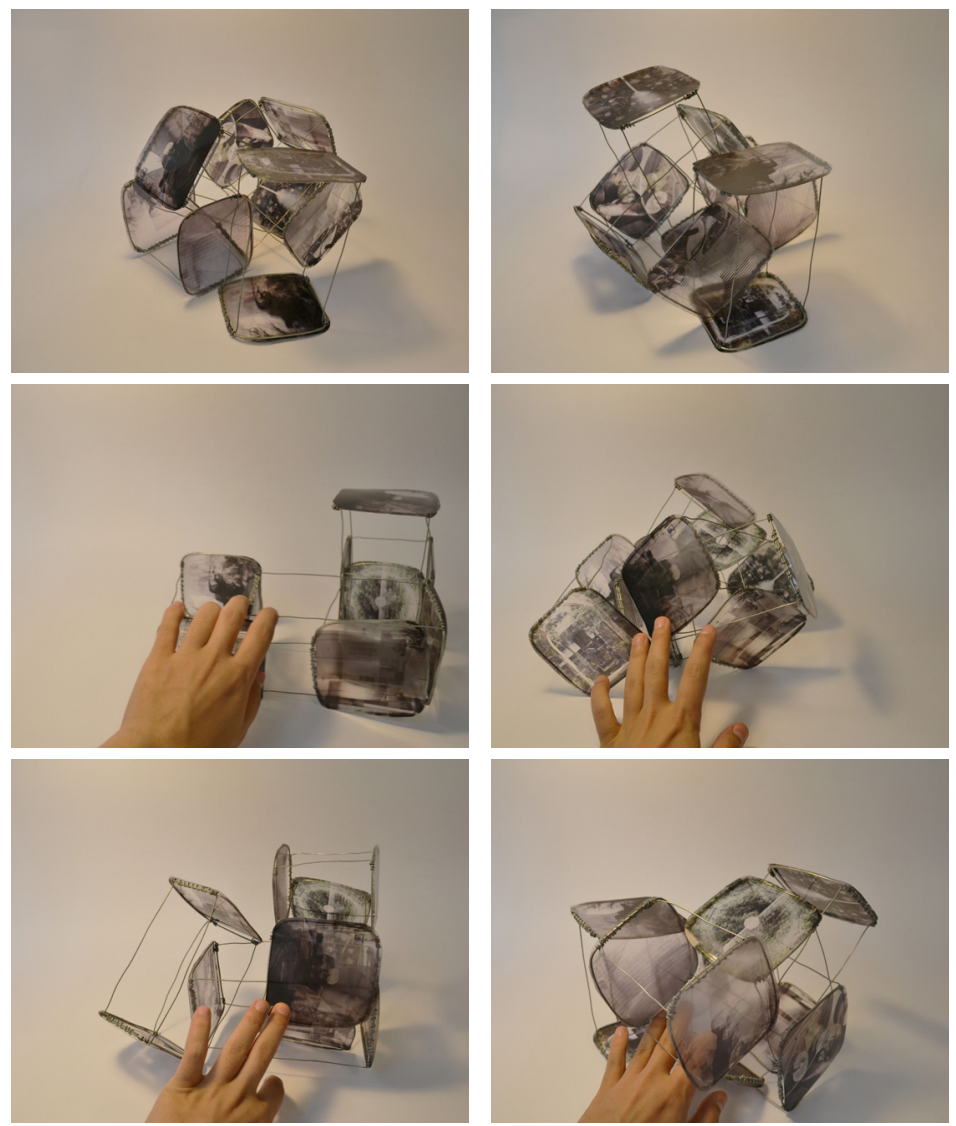

f69

Memory Overlay Wire Model 

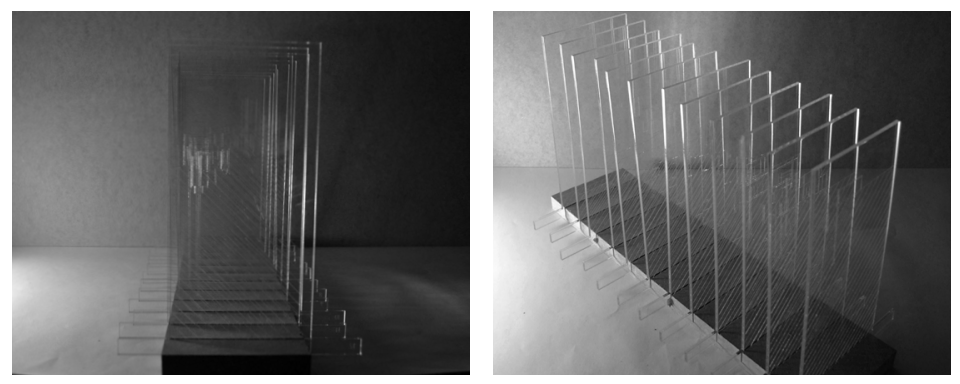

0
0
0
$\frac{u}{0}$
$\frac{1}{0}$
0
$\frac{0}{2}$
$\frac{0}{0}$
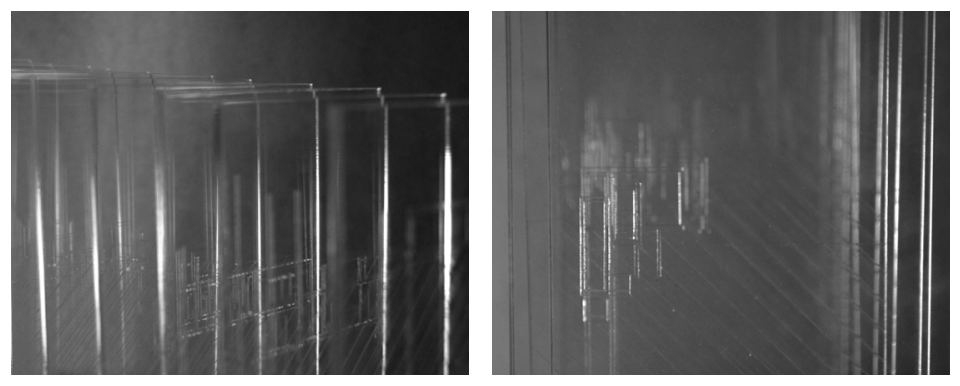

f70

Etched Section Study 
Altman, Irwin, and Setha M. Low. Place Attachment. Vol. 2, in Human Behavior and Environment: Advances in Theory and Research, by Irwin Altman, \& Joachim F. Wohlwill. New York: Plenum Press, 1992.

Audoin, Claude, and Bernard Guinot. The Measurement of Time: Time, Frequency, and the Atomic Clock. New York: Cambridge University Press, 2001.

Awotona, Adenrele, ed. Rebuilding Sustainable Communities with Vulnerable Populations after Cameras Have Gone: A Worldwide Study. Cambridge: Cambridge Scholars Publishing, 2012.

Bachelard, Gaston. The Poetics of Space. Translated by Maria Jolas. Boston: Beacon Press, 1970.

Benjamin, Walter. The Work of Art in the Age of Its Technological Reproducibility, and Other Writings on Media. Cambridge: The Belknap Press of Harvard University Press, 2008.

Bergson, Henri. Matter and Memory. Translated by Nancy Margaret Paul, \& W. Scott Palmer. London: George Allen \& Unwin, 1970.

-. Time and Free Will: An Essay on the Immediate Data of Consciousness. Translated by Frank Lubecki Pogson. New York: Dover Publications, Inc., 2001.

Böhme, Gernot. "Atmosphere as Mindful Physical Presence in Space." Building Atmosphere. Edited by Klaske Havik, Gus Tielens, \& Hans Teerds. Translated by Jeremy Gaines. Rotterdam: OASE Foundation, 2013. 21-32. 
-. "Encountering Atmospheres: A Reflection on the Concept of Atmosphere in the Work of Juhani Pallasmaa and Peter Zumthor." Building Atmosphere. Edited by Klaske Havik, Gus Tielens, \& Hans Teerds. Translated by Jeremy Gaines. Rotterdam: OASE Foundation, 2014.93-99.

-. "The Theory of Atmospheres and Its Applications ." Interstices: Journal of Architecture and Related Arts. Vol. 15. Edited by Ross Jenner and Andrew Douglas A.-Chr. Engels-Schwarzpaul. Translated by A.-Chr. Engels-Schwarzpaul. 2014. 92-99.

Bois, Yve-Alain. "Slow (Fast) Motion." In Speed Limits, edited by Jeffrey Schnapp, 122-126. Milan: Skira, 2009.

Bois, Yve-Alain. "Very Slow." In Formless, 198-204. n.d.

Bollnow, O. F. Human Space. Edited by Joseph Kohlmaier. Translated by Christine Shuttleworth. London: Hyphen Press, 2011.

Brown, Kirk Warren, and Richard M. Ryan. "The Benefits of Being Present: Mindfulness and its Role in Psychological Well-being." Journal of Personality and Social Psychology 84, no. 4 (2003): 822-848.

Bruno, Giuliana. Atlas of Emotion: Journeys in Art, Architecture, and Film. London: Verso, 2002.

Deleuze, Gilles. Cinema 1: The Movement-Image. Translated by Hugh Tomlinson, \& Barbara Habberjam. London: The Athlone Press, 1986.

Diller, Elizabeth, and Ricardo Scofidio. Flesh. New York: Princeton Architectural Press, 1994.

Duffy, Enda. The Speed Handbook: Velocity, Pleasure, Modernism. Durham: Duke Univeristy Press, 2009.

Eisenstein, Sergei M., Yve-Alain Bois, and Michael Glenny. "Montage and Architecture." Assemblage, no. 10 (1989): 110-131.

Eisenstein, Sergei. "Piranesi, or the Fluidity of Forms." Oppositions II, 1978: 83-110.

Forty, Adrian. Words and Buildings: A Vocabulary of Modern Architecture. New York: Thames \& Hudson, 2000.

Gibson, James J. The Perception of The Visual World. Boston: Houghton Mifflin, 1950.

Giedion, Sigfried. Space, Time and Architecture. Cambridge: Harvard University Press, 1959.

Gleick, James. Faster: The Acceleration of Just About Everything. Toronto: Vintage Books, 1999. 
Gumbrecht, Hans Ulrich. Production of Presence: What Meaning Cannot Convey. Stanford: Stanford University Press, 2003.

Heidegger, Martin. "Building, Dwelling, and Thinking." In Basic Writings, 343-363. New York: Harper Perennial Modern Classics, 2008.

Holl, Steven. Intertwining. New York: Princeton Architectural Press, 1996.

Holl, Steven, Juhani Pallasmaa, and Alberto Pérez Gómez. Questions of Perception: Phenomenology of Architecture. San Francisco: William Stout Publishers, 2006.

Honoré, Carl. In Praise of Slownesss: Challenging the Cult of Speed. New York: Harper Collins ebook, 2009.

Koeck, Richard. Cine-scapes: Cinematic Spaces in Architecture and Cities. Abingdon: Routledge, 2013.

Kundera, Milan. Slowness. Translated by Linda Asher. New York: Harper Perennial, 1997.

Kwinter, Sanford. Architectures of Time: Toward a Theory of the Event in Modernist Culture. Cambridge: The MIT Press, 2001.

Lefebvre, Henri. Rhythmanalysis: Space, Time and Everyday Life. Translated by Stuart Elden, \& Gerald Moore. New York: Continuum, 2004.

Lefebvre, Henri. "The Everyday and Everydayness." Yale French Studies, no. 73 (1987): 7-11.

-. The Production of Space. Translated by Donald Nicholson-Smith. Oxford: Blackwell, 1991.

Levine, Robert. A Geography of Time: The Temporal Misadventures of a Social Psychologist, or How Every Culture Keeps Time Just a Little Bit Differently keeps time just a little bit differently. New York: Basic Books, 1997.

Luca, Tiago De, and Nuno Barradas Jorge, eds. Slow Cinema. Edinburgh: Edinburgh University Press, 2012.

Lynn, Greg. "Geometry in Time." In Anyhow, edited by Cynthia C. Davidson, 164-173. Cambridge: The MIT Press, 1998.

Marinetti, F. T. "The Founding and Manifesto of Futurism." In Futurism: An Anthology, edited by Lawrence Rainey, Christine Poggi, \& Laura Wittman, 49-54. New Haven: Yale University Press, 2009. 
Merleau-Ponty, Maurice. Phenomenology of Perception.

Translated by Donald A. Landes. London:

Routledge, 2014.

-. The Visible and the Invisible. Translated by Alphonso Lingis. Evanston: Northwestern University Press, 1968.

Morris, David. The Sense of Space. Albany: State University of New York, 2004.

Nitschke, Günter. From Shinto to Ando: Studies in Architectural Anthropology in Japan. London: Academy Editions, 1993.

Pallasmaa, Juhani. "Hapticity and Time: Notes on a Fragile Architecture." The Architectural Review, 2000: 78-84.

Pallasmaa, Juhani. "Inhabiting Time." Architectural Design 86 (2016): 50-59.

Pallasmaa, Juhani. "Space, Place, Memory and Imagination: The Temporal Dimension of Existential Space." In Spatial Recall: Memory in Architecture and Landscape, edited by Marc Treib, 16-41. New York: Routledge, 2009.

-. The Architecture of Image: Existential Space in Cinema. Helsinki: Rakennustieto Publishing, 2007.

-. The Eyes of the Skin: Architecture and the Senses. London: John Wiley \& Sons Ltd., 2005.

Pallasmaa, Juhani. “The Space of Time." Oz 20 (1998): 55-57.

Pilgrim, Richard B. "Intervals ("Ma") in Space and Time: Foundations for a Religio-Aesthetic Paradigm in Japan." History of Religions 25, no. 5 (1986): 255277.

Providência, Paulo. Architectonica Percepta: Texts and Images 1989-2015. Zurich: Park Books, 2016.

Rainey, Lawrence, Christine Poggi, and Laura Wittman, eds. Futurism: An Anthology. New Haven: Yale University Press, 2009.

Rasmussen, Steen Eiler. Experiencing Architecture. Cambridge: The MIT Press, 1959.

Robinson, Sarah. Nesting: Body, Dwelling, Mind. Richmond: William Stout Publishers, 2011.

Rosa, Harmut, and William E. Scheuerman, eds. HighSpeed Society: Social Acceleration, Power, and Modernity. Philadelphia: Penn State University Press, 2010. 
Schmitz, Hermann, Rudolf Owen Müllan, and Jan Slaby. "Emotions Outside the Box-the New Phenomenology of Feeling and Corporeality." Phenomenology and the Cognitive Sciences, 2011: 242-259.

Schnapp, Jeffrey. "Fast (Slow) Motion." In Speed Limits, edited by Jeffrey Schnapp, 26-37. Milan: Skira, 2009.

Sherover, Charles M. The Human Experience of Time: The Development of its Philosophic Meaning. New York: New York University Press, 1975.

Spuybroek, Lars. The Architecture of Continuity: Essays and Conversations. Rotterdam: V2 Pub., 2008.

Tanizaki, Jun'ichirō. In Praise of Shadows. Translated by Thomas J. Harper, \& Edward G. Seidensticker. London: Johnatan Cape, 1977.

Tarfuri, Manfredo. The Sphere and the Labyrinth. Cambridge: The MIT Press, 1987.

Tarkovsky, Andrei. Sculpting in Time: Reflections on the Cinema. Translated by Kitty Hunter-Blair. Austin: University of Texas Press, 1987.

Thera, Nyanaponika. The Power of Mindfulness. San Francisco: Unity Press, 1972

Till, Jeremy. "Architecture in Space, Time." In Architecture and Anthropology, edited by Clare Melhuish, 9-13. London: Academy Editions, 1996.

Till, Jeremy. "Thick Time." In Intersections: Architectural Histories Critical Theories, edited by lain Borden, \& Rendell Jane, 283-295. London: Routledge, 2000.

Tschumi, Bernard. Architecture and Disjunction. Cambridge: The MIT Press, 1996.

-. The Manhattan Transcripts. London: Academy Editions, 1994.

Vidler, Anthony. Warped Apace: Art, Architecture, and Anxiety in Modern Culture. Cambridge: The MIT Press, 2000.

Virilio, Paul. A Landscape of Events. Translated by Julie Rose. Cambridge: The MIT Press, 2000. 

